\title{
Closures for coarse-grid simulation of fluidized gas-particle flows
}

Final technical report

\begin{tabular}{|c|c|}
\hline Report Period: & January 1, 2006 \\
\hline & June 30, 2010 \\
\hline Principal Author: & Sankaran Sundaresan \\
\hline & Department of Chemical Engineering \\
\hline & Princeton University, Princeton, NJ 08544 \\
\hline Report issued on: & July, 2010 \\
\hline Submitting organization: & Princeton University, Princeton, NJ 08544 \\
\hline DOE Topic/Area of Interest: & 11 - Coarse-grid modeling \\
\hline & DE-PS26-05NT42472-11 \\
\hline DOE-UCR Grant No: & DE-FG26-06NT42737 \\
\hline
\end{tabular}




\section{Disclaimer}

This report was prepared as an account of work sponsored by an agency of the United States Government. Neither the United States Government nor any agency thereof, nor any of their employees, makes any warranty, express or implied, or assumes any legal liability or responsibility for the accuracy, completeness, or usefulness of any information, apparatus, product, or process disclosed, or represents that its use would not infringe privately owned rights. Reference herein to any specific commercial product, process, or service by trade name, trademark, manufacturer, or otherwise does not necessarily constitute or imply its endorsement, recommendation, or favoring by the United States Government or any agency thereof. The views and opinions of authors expressed herein do not necessarily state or reflect those of the United States Government or any agency thereof. 


\begin{abstract}
Context: Gas-particle flows in fluidized beds and riser reactors are inherently unstable, and they manifest fluctuations over a wide range of length and time scales. Two-fluid models for such flows reveal unstable modes whose length scale is as small as ten particle diameters. Yet, because of limited computational resources, gas-particle flows in large fluidized beds are invariably simulated by solving discretized versions of the twofluid model equations over a coarse spatial grid. Such coarse-grid simulations do not resolve the small-scale spatial structures which are known to affect the macroscale flow structures both qualitatively and quantitatively. Thus there is a need to develop filtered two-fluid models which are suitable for coarse-grid simulations and capturing the effect of the small-scale structures through closures in terms of the filtered variables.
\end{abstract}

Project Overview: The overall objective of the project is to develop validated closures for filtered two-fluid models for gas-particle flows, with the transport gasifier as a primary, motivating example. In this project, highly resolved three-dimensional simulations of a kinetic theory based two-fluid model for gas-particle flows have been performed and the statistical information on structures in the 100-1000 particle diameters length scale has been extracted. Based on these results, closures for filtered two-fluid models have been constructed. The filtered model equations and closures have been validated against experimental data and the results obtained in highly resolved simulations of gas-particle flows. 
Broad Impact: The proposed project enables more accurate simulations of not only the transport gasifier, but also many other non-reacting and reacting gas-particle flows in a variety of chemical reactors. The results of this study are in the form of closures which can readily be incorporated into existing multi-phase flow codes such as MFIX (www.mfix.org). Therefore, the benefits of this study can be realized quickly. The training provided by this project has prepared a $\mathrm{PhD}$ student to enter research and development careers in DOE laboratories or chemicals/energy-related industries. 


\section{Table of Contents}

Page Number

Cover Page

Disclaimer $\quad$ ii

Abstract iii

$\begin{array}{ll}\text { Project Milestones } & 1\end{array}$

1. Simulations in periodic domains to extract filter size dependent closures 2

2. Simulations in periodic domains to extract filter size dependent closures $\quad 10$

3. General closure relations 12

3.1. Filtered drag coefficient 13

3.2. Filtered particle phase pressure and viscosity 15

4. Validation studies 16

5. Summary 24

6. Project participant 25

7. Published articles 25

8. Articles in preparation 25

9. Conference presentations 26

$\begin{array}{ll}\text { 10. Other presentations } & 27\end{array}$

11. References 28

Appendix A

Appendix B

Appendix C 


\section{Project Milestones}

\begin{tabular}{|c|c|c|}
\hline Milestone \# & Description & Status \\
\hline 1 & $\begin{array}{l}\text { Demonstrate capability to gather region-averaged statistics from 3-D } \\
\text { simulations of fluidization of uniformly sized particles in periodic } \\
\text { domains }\end{array}$ & achieved \\
\hline 2 & $\begin{array}{l}\text { Complete the "sensitivity" runs and expose the robustness of the } \\
\text { region-averaged statistics against small changes in secondary model } \\
\text { parameters. }\end{array}$ & achieved \\
\hline 3 & $\begin{array}{l}\text { Complete three 3-D simulations and extract filtered (i.e. region- } \\
\text { averaged) quantities for various filter sizes. }\end{array}$ & achieved \\
\hline 4 & Complete two more highly resolved simulations. & achieved \\
\hline 5 & $\begin{array}{l}\text { Complete all the highly resolved simulations for the case of uniformly } \\
\text { sized particles. }\end{array}$ & achieved \\
\hline 6 & $\begin{array}{l}\text { Complete the simulations of the filtered equations for at least two } \\
\text { different grid resolutions. }\end{array}$ & achieved \\
\hline 7 & $\begin{array}{l}\text { Complete changes in the statistics gathering program to allow for the } \\
\text { presence of the side walls. }\end{array}$ & achieved \\
\hline 8 & $\begin{array}{l}\text { Propose effective boundary conditions for the filtered equations at the } \\
\text { side walls. }\end{array}$ & achieved \\
\hline 9 & $\begin{array}{l}\text { For the case of uniformly sized particles, complete validation of the } \\
\text { filtered two-fluid model against data from highly resolved simulations. }\end{array}$ & achieved \\
\hline 10 & $\begin{array}{l}\text { Complete at least one validation of the filtered two-fluid model against } \\
\text { experimental data. }\end{array}$ & achieved \\
\hline
\end{tabular}

Project Status: We have met the project milestones listed above. In this report, we will present the results of this project in four main sections:

1. Simulations in periodic domains to extract size dependent closures

2. Wall corrections and verification of the filtered model

3. General closure relations

4. Validation simulations

The details are given below. 


\section{Simulations in periodic domains to extract filter size dependent closures}

(Note: In what follows in this section, we present only an overview of the problem addressed and highlight the main findings. A detailed report that contains all the relevant figures, tables and equations can be found in Appendix A, which is a journal manuscript prepared as a part of this project.) Chemical reactors that take the form of fluidized beds and circulating fluidized beds are widely used in energy-related and chemical process industries. ${ }^{1}$ Gas-particle flows in these devices are inherently unstable; they manifest fluctuations over a wide range of length and time scales. Analysis of the performance of large scale fluidized bed processes through computational simulations of hydrodynamics and energy/species transport is becoming increasingly common. In the present study, we are concerned with the development of hydrodynamic models which are useful for simulation of gas-particle flows in large scale fluidized processes.

The number of particles present in most gas-particle flow systems is large, rendering detailed description of the motion of all the particles and fluid elements impractical. Hence, twofluid model equations ${ }^{2-4}$ are commonly employed to probe the flow characteristics, and species and energy transport. In this approach, the gas and particle phases are treated as interpenetrating continua, and locally-averaged quantities such as the volume fractions, velocities, species concentrations and temperatures of gas and particle phases appear as dependent field variables. The averaging process leading to two-fluid model equations erases the details of flow at the level of individual particles; but their consequences appear in the averaged equations through terms for which one must develop constitutive relations. For example, in the momentum 
balance equations, constitutive relations are needed for the gas-particle interaction force and the effective stresses in the gas and particle phases.

The general form of the two-fluid model equations is fairly standard and this has permitted the development of numerical algorithms for solving them. For example, open-source packages such as MFIX ${ }^{4,5}$ and commercial software (e.g., Fluent ${ }^{\circledR}$ ) can readily be applied to perform transient integration (of the discretized forms) of the balance equations governing reactive and non-reactive multiphase flows. The results generated through such simulations are dependent on the postulated constitutive models, and a major focus of research over the past few decades has been on the improvement of these constitutive models.

Through a combination of experiments and computer simulations, constitutive relations have been developed in the literature for the fluid-particle interaction force and the effective stresses in the fluid and particle phases. In gas-particle systems, the interaction force is predominantly due to the drag force. An empirical drag law that bridges the results of Wen \& $\mathrm{Yu}^{6}$ for dilute systems and the Ergun ${ }^{7}$ approach for dense systems is widely used in simulation studies. $^{2}$ In the past decade, ab initio drag force models have also been developed via detailed simulations of fluid flow around assemblies of particles. ${ }^{8-14}$

The Stokes number associated with the particles in many gas-particle mixtures is sufficiently large that particle-particle and particle-wall collisions do occur; furthermore, when the particle volume fraction is below $\sim 0.5$, the particle-particle interactions occur largely through binary collisions. The particle phase stress in these systems is widely modeled through the kinetic theory of granular materials. 2, 15, 16 This kinetic theory approach has also been extended to systems containing mixtures of different types of particles. ${ }^{17-20}$ 
It is important to keep in mind that all these closures are derived from data or analysis of nearly homogeneous systems. Henceforth, we will refer to the two-fluid model equations coupled with constitutive relations deduced from nearly homogeneous systems as the microscopic two-fluid model equations. For example, the kinetic theory based model equations described and simulated in most of the literature references fall in this category. ${ }^{16-24,25-31}$

A practical difficulty comes about when one tries to solve these microscopic two-fluid model equations for gas-particle flows. Gas-particle flows in fluidized beds and riser reactors are inherently unstable, and they manifest inhomogeneous structures over a wide range of length and time scales. There is a substantial body of literature where researchers have sought to capture these fluctuations through numerical simulation of microscopic two-fluid model equations. Indeed, two-fluid models for such flows reveal unstable modes whose length scale is as small as ten particle diameters. ${ }^{30,31}$ This can readily be ascertained by simple simulations, as illustrated in Figure 1 in Appendix A. Transient simulations of a fluidized suspension of ambient air and typical Fluid Catalytic Cracking catalyst particles were performed (using MFIX ${ }^{4,5}$ ) in a Cartesian, two-dimensional (2-D), periodic domain at different grid resolutions; these simulations employed kinetic theory based (microscopic) two-fluid model equations (summarized in Table 1 in Appendix A). The relevant parameter values can be found in Table 2 in Appendix A. The simulations revealed that an initially homogeneous suspension gave way to an inhomogeneous state with persistent fluctuations. Snapshots of the particle volume fraction fields obtained in simulations with different spatial grid resolution are shown in Figure 1 in Appendix A. It is readily apparent that finer and finer structures are resolved as the spatial grid is refined. Statistical quantities obtained by averaging over the whole domain were found to depend on the grid resolution employed in the simulations and they became nearly grid-size 
independent only when grid sizes of the order of a few particle diameters were used (see Agrawal et al..$^{30}$ for further discussion). Thus, if one sets out to solve the microscopic two-fluid model equations for gas-particle flows, grid sizes of the order of a few particle diameters become necessary. Moreover, such fine spatial resolution reduces the numerical time steps required, further increasing the computational effort. For most devices of practical (commercial) interest, such extremely fine spatial grids and small time steps are unaffordable. ${ }^{32}$ Indeed, gas-particle flows in large fluidized beds and risers are often simulated by solving discretized versions of the two-fluid model equations over a coarse spatial grid. Such coarse grid simulations do not resolve the small-scale (i.e., sub-grid scale) spatial structures which, according to the microscopic two-fluid equations and experimental observation, do indeed exist. The effect of these unresolved structures on the structures resolved in coarse-grid simulations must be accounted for through appropriate modifications to the closures - for example, the effective drag coefficient in the coarse-grid simulations will be smaller than that in the original two-fluid model to reflect the tendency of the gas to flow more easily around the unresolved clusters ${ }^{30,31}$ than through a homogenous distribution of these particles. Qualitatively, this is equivalent to an effectively larger apparent size for the particles.

One can readily pursue this line of thought and examine the influence of these unresolved structures on the effective inter-phase transfer and dispersion coefficients which should be used in coarse-grid simulations. Inhomogeneous distribution of particles will promote bypassing of the gas around the particle-rich regions and this will necessarily decrease the effective interphase mass and energy transfer rates. Conversely, fluctuations associated with the small scale inhomogeneities will contribute to the dispersion of the particles and the gas, but this effect will be unaccounted for in the coarse-grid simulations of the microscopic two-fluid models. 
Researchers have approached this problem of treating unresolved structures through various approximate schemes. O'Brien \& Syamlal ${ }^{33}$, Boemer et al. ${ }^{34}$ and Heynderickx et al. ${ }^{35}$ pointed out the need to correct the drag coefficient to account for the consequence of clustering, and proposed a correction for the very dilute limit. Some authors have used an apparent cluster size in an effective drag coefficient closure as a tuning parameter, ${ }^{36}$ others have deduced corrections to the drag coefficient using an Energy Minimization Multi-Scale approach. ${ }^{37}$ The concept of particle phase turbulence has also been explored to introduce the effect of the fluctuations associated with clusters and streamers on the particle phase stresses. ${ }^{38,39}$ However, a systematic approach that combines the influence of the unresolved structures on the drag coefficient and the stresses has not yet emerged. The effects of these unresolved structures on inter-phase transfer and dispersion coefficients remain unexplored.

Agrawal et $a .^{30}$ showed that the effective drag law and the effective stresses, obtained by averaging (the results gathered in highly resolved simulations of a set of microscopic two-fluid model equations) over the whole (periodic) domain, were very different from those used in the microscopic two-fluid model and that they depended on size of the periodic domain. They also demonstrated that all the effects seen in the 2-D simulations persisted when simulations were repeated in three dimensions (3-D) and that both 2-D and 3-D simulations revealed the same qualitative trends. Andrews et al. ${ }^{31}$ performed many highly resolved simulations of fluidized gas-particle mixtures in a 2-D periodic domain whose total size coincided with that of the grid size in an anticipated large-scale riser flow simulation. Using these numerical results, they constructed ad hoc sub-grid models for the effects of the fine-scale flow structures on the drag force and the stresses, and examined the consequence of these sub-grid models on the outcome of the coarse-grid simulations of gas-particle flow in a large-scale vertical riser. They 
demonstrated that these sub-grid scale corrections affect the predicted large-scale flow patterns profoundly. ${ }^{31}$

Thus, it is clear that one must carefully examine whether a microscopic two-fluid model must be modified to introduce the effects of unresolved structures before embarking on coarsegrid simulations of gas-particle flows. In the study by Andrews et al. ${ }^{31}$, the filtering was done simply by choosing the filter size to be the grid size in the coarse-grid simulation of the filtered equations. Furthermore, the corrections accounting for the effects of the structures which would not be resolved in the coarse-grid simulations were extracted from highly resolved simulations performed in a periodic domain whose size was chosen to be the same as the filter size; this imposed periodicity necessarily limited the dynamics of the structures in the highly resolved structures and so the accuracy of using the sub-grid models deduced from such restrictive simulations is debatable.

In the present study, we have further extended the filtering approach and presented a methodology where computational results obtained through highly resolved simulations (in a large periodic domain) of a given microscopic two-fluid model are filtered to deduce closures for the corresponding filtered two-fluid model equations. (In this case, as the filter size is considerably smaller than the periodic domain size, the microstructures sampled in the filtered region are not constrained by the periodic boundary conditions.) These filtered closures depend on the filter size and can readily be constructed for a range of filter sizes. We found that, to a good approximation, the dimensionless filtered drag coefficient, particle phase pressure and particle phase viscosity can be treated as functions of only particle volume fraction and dimensionless filter size. The effective drag coefficient to describe the inter-phase interaction force in the filtered equations shows two distinct regimes. At particle volume fractions greater 
than about 0.075 , it follows an effective Richardson-Zaki relationship and the effective R-Z exponent and apparent terminal velocity have an understandable physical interpretation in terms of interactions between particle clusters instead of the individual particles. At low particle volume fractions, the drag coefficient shows an anomalous behavior that is consistent with the formation of larger and denser clusters with increasing particle volume fraction.

The velocity fluctuations associated with the very complicated inhomogeneous structures shown by the microscopic two-fluid simulations dictate the magnitudes of the filtered particle phase pressure and viscosity. The contributions of the kinetic theory pressure and viscosity to these filtered quantities are negligibly small and so, for practically relevant filter sizes, one need not include the filtered granular energy equation in the analysis. This, however, does not mean that the fluctuations at the level of the individual particles, which the kinetic theory strives to model, are not important at all; these fluctuations influence the inhomogeneous microstructure and their velocity fluctuations, and hence the closures for the filtered equations.

It should be emphasized that the present study does not challenge the validity of the microscopic two-fluid model equations such as the kinetic theory based equations. Instead, it uses these microscopic equations as a starting point and seeks modifications to make them suitable for coarse-grid simulations. (If a fine grid can be used to resolve all the structures contained in the microscopic two-fluid model equations, the present analysis is unnecessary; however, such high resolution is neither practical nor desirable for the analysis of the macroscale flow behavior.)

In the present study, we have also demonstrated that filtering does indeed remove small scale structures that are afforded by the microscopic two-fluid models. If filtering has been done 
in a meaningful manner, the filtered equations should yield coarser structures than the microscopic two-fluid model (from which the filtered equations were derived) even when one uses fine spatial resolution. We have demonstrated this through a 1-D linear stability analysis of the filtered two-fluid models based on various filter lengths. This analysis showed that filtering is indeed erasing the fine structure and only presenting coarser structures. (All the details are presented in a journal manuscript attached as appendix A of this report.)

It is clear from our simulation results (summarized in Appendix A) that there is a striking similarity between the 2-D and 3-D results. Although there are quantitative differences between 2-D and 3-D, the following characteristics were found to be common between them:

a) The filtered drag coefficient decreased with increasing filter size, and

b) The filtered particle phase pressure and viscosity increased with filter size.

It seems reasonable to expect that the clusters will not grow beyond some critical size; if this is indeed the case, the filtered drag coefficient, and particle phase pressure and viscosity will become nearly independent of the filter size beyond some critical value. It is important to understand if such saturation occurs and, if so, at what filter size. It is also important to incorporate the effects of bounding walls on the filtered closures as comparison of the filtered model predictions with experimental data cannot be pursued until this issue is addressed. These will be explored later in the next two sections of this report. Further details of the results obtained through simulations in periodic domains can be found in the AIChE journal manuscript ${ }^{40}$ attached in Appendix A. 


\section{Wall corrections and verification of the filtered model}

(Note: In what follows in this section, we present only an overview of the problem addressed and highlight the main findings. A detailed report that contains all the relevant figures, tables and equations can be found in Appendix B, which is a draft manuscript - to e submitted to a journal - prepared as a part of this project.) It has now been conclusively established that clusters and streamers are formed in gas-particle flows in vertical risers and they are found more frequently near the tube walls so that on an average, particle concentration is larger near the wall region. A consequence of such segregation is that the average velocity of particles and gas in the wall region can be downward even though the net flow is in the upward direction - a dilute rising core and a dense descending annular region. ${ }^{32,41-45}$ Clusters at the wall of a riser have been observed to form, descend, break-up, travel laterally from the annulus to the core and then be re-entrained in the upward flowing core. In this manner, they contribute to the internal solids mixing process within a riser. ${ }^{41}$ Consequently, it is important to incorporate the effects of bounding walls on the filtered closures as comparison of the filtered model predictions with experimental data cannot be pursued until this issue is addressed.

Thus, it is clear that one must carefully examine if the closures for the filtered model equations should be modified to introduce the effects of the bounding walls before embarking on coarse-grid simulations of gas-particle flows. In the present study, we have investigated the effect of the bounding walls on the closure relationships for the filtered two-fluid model equations through a set of 2-D (two-dimensional) flow simulations in a channel equipped with bounding walls and inlet and outlet regions. The present study reveals that: 
a. One should not use the closures from the core region of the suspension for the regions close to the wall; the filtered drag coefficient and particle phase stress terms must be allowed to depend not only on particle volume fraction and the filter size, but also on the distance from the wall. However, the wall effect should not be measured as a fraction of the channel width; instead, it should be viewed in terms of the actual distance (or made dimensionless using a length scale other than the channel width) from the wall.

b. The wall corrections to the filtered closures are nearly independent of the mean particle volume fraction in the filtering region.

c. The wall corrections to the filtered closures are nearly independent of the filter lengths considered in this study.

d. The simplest effective boundary condition for the filtered equations at the bounding walls is free-slip boundary condition.

Incorporating the distance dependence of the filtered closures as wall corrections to these closures and comparing the results from simulations in 2-D channels for various grid lengths, we have established the grid resolution independence of the filtered model extracted with 4.112 dimensionless unit-filters for grid lengths smaller than half of the filter length. The results obtained from these filtered model simulations were also comparable to those predicted by highly-resolved kinetic theory based simulations. (Here, it should be noted that kinetic theory manifested a heavy dependence on grid resolution in the same simulation geometry.) Additionally, detailed analysis of the wall correction to each term revealed that the corrections affected the results predicted by the filtered model simulations quantitatively (such as an increase or decrease in particle phase mass inventory in the bed) but did not affect the grid-resolution 
independence of these results. We have also observed that the same filtered model without wall corrections afforded grid size independence. However, its quantitative accuracy was far from being satisfactory (particularly the particle mass inventory.)

Another important observation is that, through simulations in a wider channel, we have established that the two filtered models extracted with different filter lengths (4.112 and 8.224) yielded comparable results. It should also be noted that the filtered model extracted with the dimensionless filter length of 8.224 has essentially yielded grid-resolution independent results for grid lengths smaller than half of the filter length as well.

Finally, we compared the CPU time required to run one simulation second with the filtered model equations and the highly-resolved kinetic theory model. The filtered model consistently ran must faster than the kinetic theory based model. Furthermore, larger the filter size, the faster the computations became. (We expect that this effect will be even more pronounced in 3-D simulations and with larger filter lengths.) Further details can be found in in Appendix B.

\section{General closure relations}

(Note: In what follows in this section, we present only an overview of the problem addressed and highlight the main findings. A detailed report that contains all the relevant figures, tables and equations can be found in Appendix C, which is a draft manuscript - to be submitted to a journal after further editing - prepared as a part of this project.) In our earlier work (outlined in $\S 1$ ), we have presented a methodology where computational results obtained through highly resolved simulations (in a large periodic domain) of a given microscopic two- 
fluid model are filtered to deduce closures for the corresponding filtered two-fluid model equations that are appropriate for coarse-grid simulations of gas-particle flows of moderate particle volume fractions. We have found that, to a good approximation, the dimensionless filtered drag coefficient, particle phase pressure and particle phase viscosity extracted from 2-D and 3-D simulations can be treated as functions of only particle volume fraction and dimensionless filter size.

However, the closure relationships for the drag coefficient and the effective stresses presented in our earlier work did not capture the behavior in regions of very low concentrations $\left(\phi_{s}<0.01\right)$ well and did not include high particle concentrations $\left(\phi_{s}>0.30\right)$ at all. In this study, we have investigated the region-averaged statistics in these regimes as well. We have also analyzed the extended data obtained over a wide range of filter sizes and captured them into correlations, thereby shedding some light on the asymptotic behavior at large filters. Here, we have restricted our attention to closures for the filtered drag coefficient and particle phase stress terms in flow regions far away from solid boundaries and performed all our analyses in periodic domains. (Wall correction has already been discussed in the previous section.) A summary of our findings is given below.

\subsection{Filtered drag coefficient:}

Previously, the filtered drag coefficient to describe the inter-phase interaction force in the filtered equations was studied for $\phi_{s}<0.30$ and shown to exhibit two distinct regimes. For $0.075<\phi_{s}<0.30$, it follows an effective Richardson-Zaki relationship, where the effective R-Z exponent and apparent terminal velocity have an understandable physical interpretation in terms of interactions between particle clusters instead of the individual particles. At low particle 
volume fractions, the filtered drag coefficient shows an anomalous behavior that is consistent with the formation of larger and denser clusters with increasing particle volume fraction. In both regimes, the filtered drag coefficient decreased with increasing filter size.

At extremely low particle volume fractions $\left(\sim 10^{-4}\right)$ all the curves corresponding to various filter lengths approach the same value indicating that the filtered drag coefficient is essentially independent of filter length in this regime. As the particle volume fraction increases, the filtered drag coefficient picks up a definite dependence on the filter size, manifesting a systematic decrease with increasing filter size.

In the dense regions, we have seen the filtered drag coefficient cannot be captured with a Richardson-Zaki like model reasonably well. However, the dependence of the filtered closures on filter length is still clearly observed for $0.30<\phi_{s}<0.59$. At very high particle volume fractions (for $\phi_{s}>0.59$ ) all the curves corresponding to various filter lengths approach the same value indicating that the filtered closures are essentially independent of filter length in this regime. Furthermore, the filtered drag coefficient (for all filter sizes) at very high particle concentrations correspond to those used in the microscopic model ${ }^{30,40 .}$

We have sought an internally consistent closure that combines all these regimes in a compact manner (as a function of particle volume fraction and filter length). To do this, we have analyzed the extended data obtained over a wide range of filter lengths and scaled them into correlations. (See Table 2 for 2-D closure relations that should be used in 2D coarse-grid simulations.) 
We have also extended such a study to three dimensions and constructed closure models to be used in $3 \mathrm{D}$ coarse grid simulations, see Table 3.

\subsection{Filtered particle phase pressure and viscosity:}

We have also analyzed filtered particle phase pressure and shear viscosity, whose magnitudes are dictated by the velocity fluctuations associated with the complex inhomogeneous structures shown by the 2-D and 3-D microscopic two-fluid model simulations, and have sought internally consistent constitutive relationships for these terms (as functions of particle volume fraction and filter length) for the entire particle phase volume fraction range. The filtering effect on these terms depends on the particle concentration in the filtering region.

The filtered particle phase pressure and shear viscosity increase with filter length for $\phi_{s}<0.59$, a direct consequence of the fact that the energy associated with the velocity fluctuations increases with filter length. At higher $\phi_{s}$ values, the sub-filter-scale velocity fluctuations disappear (the physical oscillations of clusters get restricted), and only the microscopic (kinetic) model particle phase pressure and shear viscosity remains. Consequently, the filtered particle phase viscosity becomes independent of the filtered size at very high $\phi_{s}$ values. The proposed 2-D and 3-D closure relations capturing the particle volume fraction and filter size dependence are summarized in Table 2 and 3, respectively. Further details can be found in a draft journal manuscript attached in Appendix C. 


\section{Validation studies}

In this part of our studies, we have performed a set of 3-D computational simulations for validation of the filtered two-fluid model against the experimental data from the riser located at the PSRI experimental research facility in Chicago (www.psrichicago.com). Note that a rigorous test of the kinetic theory model could not be done for this system due to computational limitations. This study reveals that:

a) The results obtained from the filtered model simulations are nearly axi-symmetric.

b) The 3-D filtered model equations exhibit a small dependence on grid resolution for the resolutions considered in this study. All the results presented correspond to a grid resolution of $10 \times 284 \times 16$.

c) The results predicted by the filtered models are nearly independent of the filter size.

d) In $\S 2$, we studied the effect of wall correction to the closures for the filtered model and concluded that they are necessary for capturing the results predicted by the underlying kinetic theory based two-fluid model. In the present study, we performed analogous analyses with 3-D simulations. It is found that wall corrections definitely affect the results predicted by the filtered model simulations quantitatively and qualitatively; they are necessary for capturing the experimental data.

e) We have also compared the computational data for a second PSRI case (with the same mass flux but higher gas velocity) with the experimental data and found that the filtered model (with wall corrections) does a reasonable job of capturing the experimental profiles. 
These trends are qualitatively similar to those obtained from the 2-D simulations. Further details are given below.

\section{Simulation conditions}

The experimental data that we will use for validation was carefully generated in a $14.2 \mathrm{~m}$ tall circulating fluidized bed riser with a $0.2 \mathrm{~m}$ i.d. located at the PSRI experimental research facility in Chicago (www.psrichicago.com). ${ }^{46}$ The experimental data set was specifically designed for a benchmark modeling exercise at the Eighth International Fluidization conference held in France in May 1995. Various research groups have studied the system and the detailed description of the experimental unit can be found elsewhere ${ }^{46-48 .}$ Although the majority of the previous studies with this system utilized 2-D computational simulations, a 3-D geometry is more likely to capture the flow in the experimental riser. Therefore, we have focused on 3-D simulations and used the simplified 3-D geometry recently published by Benyahia ${ }^{49}$ in our studies. Figure 1 presents this 3-D geometry where the particles (solids) enter the unit from a $0.1 \mathrm{~m}$ diameter inlet centered at an elevation of $0.15 \mathrm{~m}$ on the side wall. The inlet extends azimuthally from $\pi / 5$ to $4 \pi / 5$. The gas (air at $300 \mathrm{~K}$ ) entered mainly from the bottom of the unit. Both particle and gas phases leave the unit from a $0.2 \mathrm{~m}$ diameter outlet located at an elevation of $14 \mathrm{~m}$ and extending azimuthally from $\pi / 4$ to $3 \pi / 4$. It is important to note that the inlet and outlet regions have been idealized in our simulations and could therefore be partly responsible for the departure of the simulation results from the experimental data (see below).

In the simulations, we have considered two sets of experimental data with different main gas (actual) velocities (from the bottom of the unit); 5.2 and $11 \mathrm{~m} / \mathrm{s}$. In both cases, we used a particle phase mass flux of $489 \mathrm{~kg} /\left(\mathrm{m}^{2} \mathrm{~s}\right)$ and a particle volume fraction of 0.40 at the particles 
inlet. The flow is isothermal at an ambient temperature of $300 \mathrm{~K}$. Here, it should also be noted that the real system involved PSD but in the current study, we will only consider its mono-sized (76 $\mu \mathrm{m}$ particles) system. Table 1 presents the physical properties of the particles and the gas used in our computational simulations. We have performed our analyses in terms of dimensionless variables, and a filter size of 4.112 dimensionless units is equivalent to $2.660 \mathrm{~cm}$ for the $76 \mu \mathrm{m}$ FCC particle -ambient air system. In our simulations, we have used the 3-D filtered model equations with and without wall corrections to the constitutive models, which can be found in Table 3. See Table 4 for the wall corrections.

First, we examine the results predicted by the filtered model without wall corrections and demonstrate that the results are axi-symmetric. (For these studies, we set the gas velocity at the inlet to be $5.2 \mathrm{~m} / \mathrm{s}$.) Figure 2 presents the variation of the dimensionless time-averaged gas pressure gradient along the height of the 3-D riser taken near the wall at two different azimuthal locations. An azimuthally averaged profile is also included. The computational grid for the results presented in this figure consists of 10 cells along the radial direction (half the diameter), 16 cells in azimuthal direction and 284 uniform cells along the axial direction. (Thus, azimuthal grids 1 and 9 are located diametrically opposite from each other.) The filtered model used in this simulation was extracted with a filter length of 4.112 dimensionless units. Free-slip boundary condition was imposed for both phases at all walls. It is readily apparent in Figure 2 that the gas pressure gradient profile is axi-symmetric.

Similarly, Figure 3 (a) and (b) show the dimensionless time-averaged profiles of particle volume fraction and axial particle mass flux at an elevation of 602.9 dimensionless units $(=3.9$ m) above the solids inlet obtained from two azimuthal locations ( $180^{\circ}$ apart), respectively. Azimuthally averaged profiles are also shown. Both profiles are nearly axi-symmetric. 
Therefore, we will only present the azimuthally averaged quantities for the rest of our studies. Here, it is important to note that, for the grid resolution of $10 \times 284 \times 16$, after the gas-particle flow in the simulation domain reached its statistical steady state, the time-averaged data are collected for a period of 50-70 seconds at a frequency of $50 \mathrm{~Hz}$. (It takes about 11 days to collect the data for 50 seconds on a dual quad core Intel Xeon E5420 processor running at 2.50GHz.) The time-averaging period is judged sufficient when horizontal profiles of flow variables are nearly symmetric and axial profiles of gas pressure gradient are relatively smooth.

\section{Effect of grid resolution:}

To study the effect of grid resolution on the results predicted by the filtered model simulations, we have considered a case with three different spatial resolutions. (For these studies, we set the gas velocity to $5.2 \mathrm{~m} / \mathrm{s}$ at the inlet.) Figure 4 presents the effect of grid resolution on the dimensionless time-averaged gas pressure gradient along the height of the 3-D riser. Due to axi-symmetry presented earlier, azimuthally averaged profiles are used. The filtered model used in this simulation was extracted with a filter length of 4.112 dimensionless units. Free-slip boundary condition was imposed for both phases at all walls. It is apparent in the figure that an increase in the axial (vertical) resolution (from 284 grids to 710 grids) does not affect the pressure gradient profile, whereas an increase in azimuthal resolution (from 16 to 32 grids) slightly increases the pressure gradient at elevations $<950$ (dimensionless units). Note that the "higher resolution" cases exhibits more fluctuations in the gas pressure gradient profile due to shorter averaging period (due to computational time restriction) and are expected to smooth out with longer averaging periods. 
The grid resolution effect on the dimensionless time-averaged profiles of particle volume fraction and axial particle mass flux at an elevation of 602.9 dimensionless units $(=3.9 \mathrm{~m})$ above the solids inlet are shown in Figure 5 (a) and (b). It is apparent from these figures, as well as Figure 4, that 3-D filtered model equation extracted with a filter size of 4.112 (dimensionless units) exhibit a small dependence on grid resolution for the resolutions considered in this study; however, a grid resolution of $10 \times 284 \times 16$ will be used for the rest of our validation studies for the sake of computational ease (and because of the fact that the change in predicted value with further grid refinement was only small).

\section{Effect of the filter size:}

A set of 3-D flow simulations of the filtered models extracted with dimensionless filter sizes of $4.112,8.224$ and 16.448 were performed with a grid resolution of $10 \times 284 \times 16$. (For these studies, we set the gas velocity to $5.2 \mathrm{~m} / \mathrm{s}$ at the inlet.) Free-slip boundary condition was imposed for both phases at all walls. Figure 6 illustrates the effect of the filter size on the dimensionless time-averaged gas pressure gradient along the height of the 3-D riser. It is clearly seen that the gas pressure gradient is nearly independent of filter size for filter sizes of 4.112 and 8.224. The filtered model extracted with 16.448 filters yields slightly lower values for lower elevations in the riser. Here, it is important to note that a filter size (length) of 16.446 (dimensionless units) is equal to $\sim 10.64 \mathrm{~cm}$ for the system in this study. This filter size is meaningless for this problem (with a tube radius of $10 \mathrm{~cm}$ ), but perfectly realistic for larger systems. We have included this filtered model in our studies to determine the upper limit for the filter length that can be used in simulations for a given cross-sectional width. Yet, it is remarkable that even for this system the 16.448 filter captures the results predicted by smaller filter sizes (4.112 and 8.224) reasonably well. 
Similarly, the radial profiles of the dimensionless time-averaged particle volume fraction and axial particle mass flux at an elevation of 602.9 dimensionless units $(=3.9 \mathrm{~m})$ above the solids inlet are nearly independent of the filter size used to extract the filtered model. Collectively, these results illustrate that the solution is nearly filter size independent for the physically meaningful filters!

Inclusion of wall corrections to the closures for the filtered model equations:

In our earlier studies, we have shown that in a 2-D riser, the wall corrections to the closures for the filtered model equations are necessary for capturing the results predicted by the underlying kinetic theory based two-fluid model. In the present study, we performed analogous analyses with 3-D simulations.

We have extracted the wall corrections to the constitutive models for the filtered drag coefficient, particle phase pressure and viscosity from a 3-D kinetic theory based simulation of a riser with a square cross-section $(14.6 \mathrm{~cm} \times 914 \mathrm{~cm} \times 14.6 \mathrm{~cm})$ and adapted them for risers with circular cross-sections. We have seen that the wall corrections for the filtered drag, particle phase pressure and viscosity are similar. Therefore, we will use the same correction for all three; see Figure 8 and Table 4 for the wall correction to the filtered model closures.

Using the filtered model (corresponding to a filter size of 8.224 dimensionless units) and the wall corrections, we have studied the effect of the inclusion of the wall corrections to the filtered model closures. Figure 9 shows a comparison of the dimensionless time-averaged gas pressure gradient profile obtained from simulations of the filtered model equations with and without wall corrections and the experimental data generated from the PSRI riser. (For these studies, we set the gas velocity to $5.2 \mathrm{~m} / \mathrm{s}$ at the inlet and used the corresponding experimental 
data.) The resolution is $10 \times 284 \times 16$. It is readily apparent that the inclusion of wall corrections affects the gas pressure gradient profile predicted by the filtered model simulations quantitatively; the filtered model with wall corrections predict higher gas pressure gradients at almost all elevations. The results predicted by the filtered model with wall corrections are comparable to the experimental data for elevations $<1000$ (dimensionless units). Near the top of the riser (over 1900 dimensionless units), the filtered models predict pressure gradients much higher than the experimental data. Benyahia ${ }^{49}$ reported similar results with EMMS model in the same system and attributed this behavior to particles (solids) backmixing caused by the abrupt blind-tee used computationally instead of the smooth elbow exit design used experimentally.

The dimensionless time-averaged radial profiles of particle volume fraction and axial particle mass flux at an elevation of 602.9 dimensionless units $(=3.9 \mathrm{~m})$ above the solids inlet obtained with the filtered closures (8.224) with and w/o wall corrections are presented in Figure 10(a) and (b), respectively. The experimental data are also shown for comparison. In Figure 10(a), we can clearly see that inclusion of wall correction dramatically increases the particle phase concentration in the vicinity of the side wall, and it also increases the particle concentration slightly in the core region. In the core region, the filtered model without wall correction predicts the experimental data better than the model with wall correction; whereas in the wall region, the latter yields a better prediction. As for the radial profile of axial particle mass flux (see Figure 10(b)), the filtered model with wall correction predicts the experimental data better than the filtered model without wall corrections although the downward flux has not been captured by either model. This could be a consequence of the free-slip wall condition we have used in our studies and should be further investigated. Although no experimental data is available for the radial superficial gas velocity profile, we have compared the results predicted 
by the filtered model with and without wall corrections (see Figure 10(c)); inclusion of wall corrections result in higher superficial gas velocities in the core and lower velocities in the vicinity of the wall.

To summarize, the inclusion of wall corrections affects the results predicted by the filtered model simulations quantitatively and qualitatively and is necessary for capturing the experimental data.

\section{A comparison with a second set of experimental data:}

We have also compared the computational data predicted by the filtered model with wall corrections for a second PSRI case (with the same mass flux but with a higher gas velocity at the inlet, $11 \mathrm{~m} / \mathrm{s}$ ) with the experimental data. The filtered model was extracted with a filter size of 8.224 dimensionless units. Figure 11 presents a comparison of the dimensionless time-averaged gas pressure gradient profile obtained from the simulation of the filtered model equations with wall corrections and the experimental data generated from the PSRI riser. The spatial resolution is $10 \times 284 \times 16$. It is readily apparent that the results predicted by the filtered model with wall corrections are comparable to the experimental data for elevations $<700$ (dimensionless units). However, the filtered model predicts lower pressure gradients at lower elevations. As noted earlier, near the top of the riser (over 1900 dimensionless units), the filtered model predicts pressure gradients much higher than the experimental data, which is believed to be due to abrupt blind-tee outlet used in simulations.

The dimensionless time-averaged radial profiles of particle volume fraction and axial particle mass flux at an elevation of 602.9 dimensionless units $(=3.9 \mathrm{~m})$ above the solids inlet obtained with the filtered closures (8.224) and the experimental data are presented in Figure 
12(a) and (b), respectively. The filtered model yielded a radial particle volume fraction profile comparable to the experimental profile close to the wall but slightly underpredicted the particle concentration in the core region. As for the radial profile of axial particle mass flux (see Figure 12 (b)), the filtered model with wall correction captured the experimental finding that the maximum mass flux was not in the core region, however, the location of maximum flux, at the wall, is different from the experimental one, which is slightly away from the wall. We suspect that this might have caused by the free-slip BC we assumed in our studies. As mentioned earlier, this needs to be further investigated.

\section{Summary}

We have performed a set of 3-D computational simulations for validation of the filtered two-fluid model against the experimental data from the PSRI riser. This study has revealed that the 3-D filtered model equations exhibit a small dependence on grid resolution for the resolutions considered in this study; however, a grid resolution of $10 \times 284 \times 16$ was used for most of our studies for the sake of computational ease. We have shown that the results predicted by the filtered models are nearly filter size independent and that wall corrections definitely affect the results predicted by the filtered model simulations quantitatively and qualitatively and are necessary for capturing the experimental data. We have also compared the computational data for a second PSRI case (with the same mass flux but higher gas velocity) with the experimental data and shown that the filtered model (with wall corrections) has the capability to capture the experimental profiles. 


\section{Project participant}

Ms. Yesim Igci (graduate student at Princeton University) worked with the PI on this project. All the code development and analysis of results were performed by her. She will be completing her $\mathrm{PhD}$ shortly.

\section{Published articles}

Y. Igci, A. T. Andrews IV, S. Pannala, T. O’Brien \& S. Sundaresan, Filtered two-fluid models for fluidized gas-particle suspensions. AIChE J., 54, 1431-1448 (2008). See Appendix A.

\section{Articles in preparation}

The manuscript listed as Appendix B has been submitted for publication. The draft manuscript included as Appendix $\mathrm{C}$ will be revised and submitted for publication shortly. Finally, a manuscript based on the validation results discussed in the main text of this report is also under preparation. 


\section{Conference presentations}

1. S. Sundaresan, A. T. Andrews IV \& Y. Igci, "Coarse-graining two-fluid models for fluidized gas particle suspensions," The 231st ACS National Meeting, Atlanta, GA, March 28, 2006.

2. S. Sundaresan, A. T. Andrews IV, Y. Igci, S. Pannala \& T. O'Brien, "Coarse-graining two-fluid models for fluidized gas particle suspensions," Fifth World Congress on Particle Technology, Orlando, FL, April 24, 2006.

3. S. Sundaresan, , Y. Igci, A. T. Andrews IV, T. O’Brien \& S. Pannala, “Filtered TwoFluid Models for Gas-Particles Flows," The AIChE Annual Meeting, San Francisco, CA November 11-13, 2006.

4. Y. Igci, S. Sundaresan, S. Pannala, T. O’Brien \& R. Breault, “Coarse-graining two-fluid models for fluidized gas particle suspensions," Fifth International Conference on CFD in the Process Industry, CSIRO, Melbourne, Australia, Dec 13-15, 2006. (Includes a Proceedings manuscript.)

5. Y. Igci \& S. Sundaresan, "Closures for filtered two-fluid models for gas-particle flows," paper presented at the AICHE Annual Meeting, November 4-9, 2007, Salt Lake City, UT.

6. Y. Igci \& S. Sundaresan, "Coarse-grid simulation of fluidized gas-particle flows," paper presented at the AICHE Annual Meeting, November 16-21, 2008, Philadelphia, PA.

7. Y. Igci \& S. Sundaresan, "Filtered two-fluid models for fluidized gas-particle flows," paper presented at the AICHE Annual Meeting, November 8-13, 2009, Nashville, TN. 


\section{Other Presentations}

A presentation based on this work was made at the UCR-HBCU conference in Pittsburgh, June 2007. This work was also the topic of D.B. Robinson Distinguished lecture that SS presented in the Chemical Engineering Department, University of Alberta (April 2007) and seminars presented by SS in the Mechanical Engineering Department, California Institute of Technology (May 2007) and the Chemical Engineering Department, University of Florida (October 2007). This work was also in the theme of the 2009 Burgers Lecture that SS delivered at The Burgers Centre in the Netherlands (Jan 2009). SS delivered a seminar at the Chemical Engineering Department of the University of Minnesota on this topic (February 2009), a keynote lecture at the World Congress in Montreal (August 2009) and the $5^{\text {th }}$ Sino-US Conference on Chemical Engineering in Beijing (October 2009). The Neal R. Amundson Lecture presented at the University of Houston (Jan 2010) featured the results of this project. 


\section{References}

1. Grace JR, Bi H. In: Grace JR, Avidan AA, Knowlton TM, eds. Circulating Fluidized Beds. New York, NY: Blackie Academic \& Professional; 1997:1-20.

2. Gidaspow D. Multiphase Flow and Fluidization. San Diego, CA: Academic Press; 1994.

3. Jackson R. The Dynamics of Fluidized Particles: Cambridge University Press; 2000.

4. Syamlal M, Rogers W, O'Brien TJ. MFIX Documentation. Morgantown, WV: U.S. Department of Energy, Federal Energy Technology Center;1993.

5. Syamlal M. MFIX Documentation: Numerical Techniques1998. DOE/MC-31346-5824. NTIS?DE98002029.

6. Wen CY, Yu YH. Mechanics of Fluidization. Chem Eng Prog S Ser. 1966;62:100-111.

7. Ergun S. Fluid flow through packed columns. Chem Eng Prog. 1952;48:89.

8. Hill RJ, Koch DL, Ladd AJC. The first effects of fluid inertia on flow in ordered and random arrays of spheres. J Fluid Mech. 2001;448:213-241.

9. Hill RJ, Koch DL, Ladd AJC. Moderate-Reynolds-number flows in ordered and random arrays of spheres. J Fluid Mech. 2001;448:243-278.

10. Wylie JJ, Koch DL, Ladd AJC. Rheology of suspensions with high particle inertia and moderate fluid inertia. J Fluid Mech. 2003;480:95-118.

11. Kandhai DJJ, Derksen JJ, van der Akker HEA. Interphase drag coefficients in gas-solid flows. AIChE J. 2003;49:1060-1065.

12. Li J, Kuipers JAM. Gas-particle interactions in dense gas-fluidized beds. Chem Eng Sci. 2003;58:711-718. 
13. van der Hoef MA, Boetstra R, Kuipers JAM. Lattice Boltzmann simulations of low Reynolds number flow past mono- and bi-disperse arrays of spheres: results for permeability and drag forces. J Fluid Mech. 2005;528:233-254.

14. Benyahia S, Syamlal M, O'Brien TJ. Extension of Hill-Koch-Ladd drag correlation over all ranges of Reynolds number and solids volume fraction. Powder Technol. 2006;162:166-174.

15. Gidaspow D, Jung J, Singh RK. Hydrodynamics of fluidization using kinetic theory: an emerging paradigm 2002 Flour-Daniel lecture. Powder Technol. 2004;148:123-141.

16. Koch DL, Sangani AS. Particle pressure and marginal stability limits for a homogeneous monodisperse gas fluidized bed: kinetic theory and numerical simulations. J Fluid Mech. 1999;400:229-263.

17. Huilin L, Yurong H, Gidaspow D, Lidan Y, Yukun Q. Size segregation of binary mixture of solids in bubbling fluidized beds. Powder Technol. 2003;134:86-97.

18. Iddir $\mathrm{H}$, Arastoopour $\mathrm{H}$, Hrenya $\mathrm{CM}$. Analysis of binary and ternary granular mixtures behavior using the kinetic theory approach. Powder Technol. 2005;151:117-125.

19. Arnarson BO, Jenkins JT. Binary mixtures of inelastic spheres: Simplified constitutive theory. Phys Fluids. 2004;16:4543-4550.

20. Jenkins J, Mancini F. Kinetic theory for binary mixtures of smooth, nearly elastic spheres. Phys Fluids A-Fluid. 1989;1:2050.

21. Benyahia S, Arastoopour H, Knowlton TM, Massah H. Simulation of particles and gas flow behavior in the riser section of a circulating fluidized bed using the kinetic theory approach for the particulate phase. Powder Technol. 2000;112:24-33. 
22. Ding J, Gidaspow D. A Bubbling Fluidization Model Using Kinetic-Theory of Granular Flow. AIChE J. 1990;36:523-538.

23. Goldschmidt MJV, Kuipers JAM, van Swaaij WPM. Hydrodynamic modeling of dense gas-fluidized beds using the kinetic theory of granular flow: Effect of restitution coefficient on bed dynamics. Chem Eng Sci. 2001;56:571-578.

24. Neri A, Gidaspow D. Riser hydrodynamics: Simulation using kinetic theory. AIChE J. 2000;46:52-67.

25. Pita JA, Sundaresan S. Gas-Solid Flow in Vertical Tubes. AIChE J. 1991;37:1009-1018.

26. Pita JA, Sundaresan S. Developing Flow of a Gas-Particle Mixture in a Vertical Riser. AIChE J. 1993;39:541-552.

27. Louge M, Mastorakos E, Jenkins JT. The role of particle collisions in pneumatic transport. J Fluid Mech. 1991;231:345-359.

28. Sinclair JL, Jackson R. Gas-particle flow in a vertical pipe with particle-particle interactions. AIChE J. 1989;35:14731486.

29. Lun CKK, Savage SB, Jeffrey DJ, Chepurniy N. Kinetic theories of granular flows: inelastic particles in Couette flow and slightly inelastic particles in a general flow field. $J$ Fluid Mech. 1984;140:223-256.

30. Agrawal K, Loezos PN, Syamlal M, Sundaresan S. The role of meso-scale structures in rapid gas-solid flows. J Fluid Mech. 2001;445:151-185.

31. Andrews IV AT, Loezos PN, Sundaresan S. Coarse-Grid Simulation of Gas-Particle Flows in Vertical Risers. Ind Eng Chem Res. 2005;44:6022-6037.

32. Sundaresan S. Modeling the Hydrodynamics of Multiphase Flow Reactors: Current Status and Challenges. AIChE J. 2000;46:1102-1105. 
33. O'Brien TJ, Syamlal M. Particle cluster effects in the numerical simulation of a circulating fluidized bed. In: Avidan A, ed. Circulating Fluidized Bed Technology. IV. Proceedings of the Fourth International Conference on Circulating Fluidized Beds. Hidden Valley Conference Center, Somerset, PAAugust 1-5, 1993.

34. Boemer A, Qi H, Hannes J, Renz U. Modelling of solids circulation in a fluidized bed with Eulerian approach. Paper presented at: 29th IEA-FBC MeetingNov. 24-26, 1994; Paris, France.

35. Heynderickx GJ, Das AK, De Wilde J, Marin GB. Effect of Clustering on Gas-Solid Drag in Dilute Two-Phase Flow. Ind Eng Chem Res. 2004;43:4635-4646.

36. McKeen T, Pugsley T. Simulation and experimental validation of a freely bubbling bed of FCC catalyst. Powder Technol. 2003;129:139-152.

37. Yang N, Wang W, Ge W, Wang L, Li J. Simulation of Heterogeneous Structure in a Circulating Fluidized-Bed Riser by Combining the Two-Fluid with the EMMS Approach. Ind Eng Chem Res. 2004;43:5548-5561.

38. Dasgupta S, Jackson R, Sundaresan S. Turbulent gas-particle flow in vertical risers. AIChE J. 1994;40:215-228.

39. Hrenya CM, Sinclair JL. Effects of Particle-Phase Turbulence in Gas-Solid Flows. AIChE J. 1997;43:853-869.

40. Igci Y, Andrews IV AT, Sundaresan S, Pannala S, O'Brien T. Filtered Two-Fluid Models for Fluidized Gas-Particle Suspensions. AIChE J. 2008;54:1431-1448.

41. Harris AT, Davidson JF, Thorpe RB. The prediction of particle cluster properties in the near wall region of a vertical riser (200157). Powder Technol. 2002;127:128-143. 
42. Zou B, Li H, Xia Y, Ma X. Cluster structure in a circulating fluidized bed. Powder Technol. 1994;78:173-178.

43. Miller A, Gidaspow D. Dense, Vertical Gas-Solid Flow in a Pipe. AIChE J. 1992;38:1801-1815.

44. Weinstein H, Shao M, Schnitzlein M. Radial variation in solid density in high velocity fluidization. In: Basu P, ed. Circulating Fluidized Bed Technology. Elmsford, NY: Pergamon; 1986:201.

45. Bader R, Findlay J, Knowlton T. Gas/Solid flow patterns in a 30.5-cm diameter circulating fluidized bed. In: Basu P, Large JF, eds. Circulating Fluidized Bed Technology. Vol II. Elmsford, NY: Pergamon; 1988:123-137.

46. Karri SBR, Knowlton T. PSRI Challenge Problem 1, Workshop 3 Modeling Test. Paper presented at: At the Eighth International Conference on Fluidization1995; Tour, France.

47. Sun B, Gidaspow D. Computation of Circulating Fluidized-Bed Riser Flow for the Fluidization VIII Benchmark Test. Ind Eng Chem Res. 1999;38:787-792.

48. Huilin L, Qiaoqun S, Yurong H, Yongli S, Ding J, Xiang L. Numerical Study of particle cluster flow in risers with cluster-based approach. Chem Eng Sci. 2005;60:6757-6767.

49. Benyahia S. On the Effect of Subgrid Drag Closures. Ind Eng Chem Res. 2010;49:51225131.

50. Zhang DZ, VanderHeyden WB. The effects of mesoscale structures on the macroscopic momentum equations for two-phase flows. Int J Multiphas Flow. 2002;28:805-822.

51. Li J. Multi-scale modeling and method of energy minimization. Beijing1987. 
52. Li J, Tung Y, Kwauk M. Energy Transport and regime transition of particle-fluid twophase flow. In: Basu P, Large JF, eds. Circulating fluidized bed technology II. Oxford: Pergamon Press; 1988:75.

53. Li J, Cheng C, Zhang Z, Yuan J. The EMMS model, its application, development and updated concepts. Chem Eng Sci. 1999;54:5409-5425.

54. Li J, Zhang Z, Ge W, Sun Q, Yuan J. A simple variation criterion for turbulent flow in pipe. Chem Eng Sci. 1999;54:1151-1154.

55. Li J, Kwauk M. Particle-fluid two-phase flow energy-minimization multi-scale method. Beijing: Metallurgical Industry Press; 1994.

56. Wang W, Li J. Simulation of gas-solid two-phase flow by a multi-scale CFD approachExtension of the EMMS model to the sub-grid level. Chem Eng Sci. 2007;62:208-231.

57. Wang J, Ge W. Multi-scale analysis on particle-phase stresses of coarse particles in bubbling fluidized beds. Chem Eng Sci. 2006;61:2736-2741.

58. Yang N, Wang W, Ge W, Li J. CFD simulation of concurrent-up gas-solid flow in circulating fluidized beds with structure dependent drag coefficient. Chem Eng J. 2003;96:71-80.

59. Lu B, Wang W, Li J. Searching for a mesh-independent sub-grid model for CFD simulation of gas-solid riser flows. Chem Eng Sci. 2009;64:3437-3447.

60. Wang J. High-resolution Eulerian simulation of RMS of solid volume fraction fluctuation and particle clustering characteristics in a CFB riser. Chem Eng Sci. 2008;63:3341-3347.

61. Lu B, Wang W, Li J, et al. Multi-scale CFD simulation of gas-solid flow in MIP reactors with a structure-dependent drag model. Chem Eng Sci. 2007;62:5487-5494. 
62. Chalermsinsuwan B, Piumsomboon P, Gidaspow D. Kinetic theory based computation of PSRI riser: Part I—Estimate of mass transfer coefficient. Chem Eng Sci. 2009;64:11951211.

63. Wang W, Lu B, Li J. Choking and flow regime transitions: Simulation by a multi-scale CFD approach. Chem Eng Sci. 2007;62:814-819.

64. Wang J, Ge W, Li J. Eulerian simulation of heterogeneous gas-solid flows in CFB risers: EMMS-based sub-grid scale model with a revised cluster description. Chem Eng Sci. 2008;63:1553-1571.

65. Wang J. A review of Eulerian Simulation of Geldart A Particles in Gas-Fluidized Beds. Ind Eng Chem Res. 2009;48:5567-5577. 
Table 1: Physical properties of gas and solids

\begin{tabular}{lll}
\hline$d_{p}$ & Particle diameter & $7.6 \times 10^{-6} \mathrm{~m}$ \\
$\rho_{\mathrm{s}}$ & Particle density & $1712 \mathrm{~kg} / \mathrm{m}^{3}$ \\
$\rho_{g}$ & Gas density & Computed using \\
& ideal gas law $\left(\sim 1.3 \mathrm{~kg} / \mathrm{m}^{3}\right)$ & \\
$\mu_{g}$ & Gas viscosity & $1.8 \times 10^{-5} \mathrm{~kg} / \mathrm{m} \cdot \mathrm{s}$ \\
$\mathrm{g}$ & Gravitational acceleration & $9.80665 \mathrm{~m} / \mathrm{s}^{2}$ \\
$\mathrm{v}_{\mathrm{t}}$ & Terminal settling velocity & $0.251875 \mathrm{~m} / \mathrm{s}$ \\
$\frac{\mathrm{v}_{\mathrm{t}}^{2}}{\mathrm{~g}}$ & Characteristic length & $0.00646918 \mathrm{~m}$ \\
$\frac{\mathrm{v}_{\mathrm{t}}}{\mathrm{g}}$ & Characteristic time & $0.0256841 \mathrm{~s}$ \\
$\rho_{\mathrm{s}} \mathrm{v}_{\mathrm{t}}^{2}$ & Characteristic stress & $108.611 \mathrm{~kg} / \mathrm{m} \cdot \mathrm{s}^{2}$ \\
& & \\
\hline
\end{tabular}


Table 2: 2-D Filtered closures without wall corrections

Filter (region) length: $\mathrm{Fr}_{f}^{-1}=g \Delta_{f} / \mathrm{v}_{t}^{2}$

\section{2-D Filtered drag coefficient:}

$$
\overline{\beta_{\text {filtered }}}=\frac{\left(\exp \left(\text { Function }_{\text {total }}\right)\right) \rho_{s} \phi_{s}\left(1-\phi_{s}\right) g}{\mathrm{v}_{\mathrm{t}}}
$$

where

Function $_{\text {total }}=$ Function $_{\text {dense-moderate }}\left(1-\right.$ factor $\left._{\text {dilute }}\right)+$ Function $_{\text {microscopic }}$ factor $_{\text {dilute }}$

Function $_{\text {dense-moderate }}=$ Function $_{\text {moderate }}$ factor $_{\text {dense }}+$ Function $_{\text {microscopic }}\left(1-\right.$ factor $\left._{\text {dense }}\right)$

Function $_{\text {moderate }}=\left(a_{\text {Function }}\right.$ asymptotic $+(1-a)$ Function $\left._{\text {microscopic }}\right)\left(1-0.606\left(1-\phi_{s}\right)^{125}\right)$

Function $_{\text {asymptotic }}=\ln \left(\frac{\overline{\beta_{\text {asymptotic }}} \mathrm{V}_{\mathrm{t}}}{\rho_{s} g \phi_{s}\left(1-\phi_{s}\right)}\right)=-4.19 \ln \left(1-\phi_{s}\right)-1.73$ for $0.05<\phi_{s}<0.30$

$a=\left\{\begin{array}{l}-0.0350 F r_{f}^{-2}+0.300 F r_{f}^{-1}, F r_{f}^{-1}<4.00 \\ -1.44 F r_{f}+1.00, F r_{f}^{-1} \geq 4.00\end{array}\right.$

factor $_{\text {dense }}=\frac{1}{1+1.23 \times 10^{-4}\left(1-\phi_{s}\right)^{-15.5}}$

factor $_{\text {dilute }}=\left(1-\phi_{s}\right)^{b}$

$b=\left\{\begin{array}{l}292 F r_{f}^{-1}, F r_{f}^{-1}<0.500 \\ \frac{1440}{1+\exp \left(-0.560 F r_{f}^{-1}+2.47\right)}, F r_{f}^{-1} \geq 0.500\end{array}\right.$

Function $_{\text {microscopic }}$ corresponds to the filtered drag coefficient extracted with a filter length equal to the grid length used in the 2-D kinetic theory based simulations with Wen-Yu drag; the grid length is 0.257 (dimensionless unit). The equations given below are the simplest fits that capture the curve in Figure 2 (a) truthfully. 


\section{Table 2 - continued}

$$
\begin{aligned}
\text { Function }_{\text {microscopic }}= & \ln \left(\frac{\overline{\beta_{\text {microscopic }}} \mathrm{V}_{\mathrm{t}}}{\rho_{s} g \phi_{s}\left(1-\phi_{s}\right)}\right) \\
= & \left\{\begin{array}{l}
0.230\left(1-\phi_{s}\right)^{76.6}+0.0914\left(1-\phi_{s}\right)^{9770}, \phi_{s}<0.001 \\
0.180\left(1-\phi_{s}\right)^{-10.6}+0.0720\left(1-\phi_{s}\right)^{800}, 0.001 \leq \phi_{s}<0.03 \\
0.550\left(\ln \left(1-\phi_{s}\right)\right)^{2}-2.82 \ln \left(1-\phi_{s}\right)+0.162, \phi_{s} \geq 0.03
\end{array}\right.
\end{aligned}
$$

\section{2-D Filtered particle phase pressure:}

$$
\frac{\overline{p_{s, \text { filtered }}}}{\rho_{s} \mathrm{v}_{\mathrm{t}}^{2}}=\left\{\begin{array}{l}
\frac{\overline{p_{s, \text { kinetic }}}}{\rho_{s} \mathrm{v}_{\mathrm{t}}^{2}}+\text { Factor }_{p s} \phi_{s}\left(1+\phi_{s}-11 \phi_{s}^{2}+10.9 \phi_{s}^{3}\right), \phi_{s} \leq \phi_{s, \text { critical } 1} 1 \\
\frac{p_{s, \text { kinetic }}}{\rho_{s} \mathrm{v}_{\mathrm{t}}^{2}}, \phi_{s}>\phi_{s, \text { critical_1 }}
\end{array}\right.
$$

where $\phi_{s, \text { critical_1 }}$ is defined as:

Factor $_{p s} \phi_{s}\left(1+\phi_{s}-11 \phi_{s}^{2}+10.9 \phi_{s}^{3}\right)=0$ at $\phi_{s}=\phi_{s, \text { critical } \_1}$

Factor $_{p s}=\left\{\begin{array}{l}0.0300 F r_{f}^{-2}+0.250 F r_{f}^{-1}, F_{f}^{-1}<4.80 \\ 0.438 F r_{f}^{-0.881}+0.138, F r_{f}^{-1} \geq 4.80\end{array}\right.$

The kinetic model term in these models can be taken care of in two ways:

1. Also solve the granular energy equation

2. Simply add the curve fit for the kinetic particle phase pressure.

$$
\frac{\overline{p_{s, k i n e t i c}}}{\rho_{s} \mathrm{v}_{\mathrm{t}}^{2}}=\left\{\begin{array}{l}
-10.4 \phi_{s}^{2}+0.310 \phi_{s}, \phi_{s} \leq 0.0131 \\
-0.185 \phi_{s}^{3}+0.0660 \phi_{s}^{2}-0.000183 \phi_{s}+0.00232,0.0131<\phi_{s} \leq 0.290 \\
-0.00978 \phi_{s}+0.00615,0.290<\phi_{s} \leq 0.594 \\
-6.62 \phi_{s}^{3}+49.5 \phi_{s}^{2}-50.3 \phi_{s}+13.8, \phi_{s}>0.594
\end{array}\right.
$$




\section{Table 2 - continued}

\section{2-D Filtered particle phase (shear) viscosity:}

$$
\frac{\overline{\mu_{s, \text { filtered }} g}}{\rho_{s} \mathrm{v}_{\mathrm{t}}^{3}}=\left\{\begin{array}{l}
\frac{\overline{\mu_{s, \text { kinetic }}} g}{\rho_{s} \mathrm{v}_{\mathrm{t}}^{3}}+\text { Factor }_{\mu s} \phi_{s}\left(1+1.7 \phi_{s}-9 \phi_{s}^{2}+6 \phi_{s}^{3}\right), \phi_{s} \leq \phi_{s, \text { rritical } 2} \\
\frac{\mu_{s, \text { kinetic }} g}{\rho_{s} \mathrm{v}_{\mathrm{t}}^{3}}, \phi_{s}>\phi_{s, \text { rritical } 22}
\end{array}\right.
$$

where $\phi_{s, \text { critical_2 }}$ is defined as:

Factor $_{\mu s} \phi_{s}\left(1+1.7 \phi_{s}-9 \phi_{s}^{2}+6 \phi_{s}^{3}\right)=0$ at $\phi_{s}=\phi_{s, \text { critical_2 }}$

Factor $_{\mu s}=0.192 \mathrm{Fr}_{f}^{-1.25}$

The kinetic model term in these models can be taken care of in two ways:

1. Also solve the granular energy equation.

2. Simply add the curve fit for the kinetic particle phase viscosity.

$$
\overline{\frac{\mu_{s, \text { kinetic }} g}{\rho_{s} \mathrm{v}_{\mathrm{t}}^{3}}}=\left\{\begin{array}{l}
1720 \phi_{s}^{4}-215 \phi_{s}^{3}+9.81 \phi_{s}^{2}-0.207 \phi_{s}+0.00254, \phi_{s} \leq 0.0200 \\
2.72 \phi_{s}^{4}-1.55 \phi_{s}^{3}+0.329 \phi_{s}^{2}-0.0296 \phi_{s}+0.00136,0.0200<\phi_{s} \leq 0.200 \\
-0.0128 \phi_{s}^{3}+0.0107 \phi_{s}^{2}-0.0005 \phi_{s}+0.000335,0.200<\phi_{s} \leq 0.609 \\
23.6 \phi_{s}^{2}-28.0 \phi_{s}+8.30, \phi_{s}>0.609
\end{array}\right.
$$


Table 3: 3-D Filtered closures (without wall corrections)

\section{3-D Filtered drag coefficient:}

$\overline{\beta_{\text {filtered }}}=\frac{\left(\exp \left(\text { Function }_{\text {total }}\right)\right) \rho_{s} \phi_{s}\left(1-\phi_{s}\right) g}{\mathrm{v}_{\mathrm{t}}}$

where

Function $_{\text {total }}=$ Function $_{\text {dense-moderate }}\left(1-\right.$ factor $\left._{\text {dilute }}\right)+$ Function $_{\text {microscopic }}$ factor $_{\text {dilute }}$

Function $_{\text {dense-moderate }}=$ Function $_{\text {moderate }}$ factor $_{\text {dense }}+$ Function $_{\text {microscopic }}\left(1-\right.$ factor $\left._{\text {dense }}\right)$

Function $_{\text {moderate }}=\left(a\right.$ Function $_{\text {asymptotic }}+(1-a)$ Function $\left._{\text {microscopic }}\right)\left(1-\phi_{s}\right)^{-0.200}$

Function $_{\text {asymptotic }}=\ln \left(\frac{\overline{\beta_{\text {asymptotic }}} \mathrm{v}_{\mathrm{t}}}{\rho_{\mathrm{s}} \phi_{s}\left(1-\phi_{s}\right) g}\right)$

$$
=5.24\left(\ln \left(1-\phi_{s}\right)\right)^{2}-1.01 \ln \left(1-\phi_{s}\right)-1.25,0.03<\phi_{s}<0.35
$$

$a=\left\{\begin{array}{l}-0.140 F r_{f}^{-2}+0.664 F r_{f}^{-1}, F r_{f}^{-1} \leq 2.34 \\ -0.542 F r_{f}^{1.10}+1.00, F r_{f}^{-1}>2.34\end{array}\right.$

factor $_{\text {dense }}=\frac{1}{1+1.38 \times 10^{-9}\left(1-\phi_{s}\right)^{-35.0}}$

factor $_{\text {dilute }}=\left(1-\phi_{s}\right)^{b}$

$b=120\left(1-\exp \left(-0.716 \mathrm{Fr}_{f}^{-1}+0.000588\right)\right)$

Function $_{\text {microscopic }}$ corresponds to the filtered drag coefficient obtained with a filter size equal to the grid size used in the 3-D kinetic theory based simulations with Wen-Yu drag; the grid size is 0.257 dimensionless units. The equations given below are the simplest fits that capture the curve in Figure 14 (a) truthfully.

Function $_{\text {microscopic }}=\ln \left(\frac{\overline{\beta_{\text {microscopic }}} \mathrm{v}_{\mathrm{t}}}{\rho_{\mathrm{s}} \phi_{s}\left(1-\phi_{s}\right) g}\right)=\left\{\begin{array}{l}0.0390\left(1-\phi_{s}\right)^{-22.1}+0.438\left(1-\phi_{s}\right)^{376}, \phi_{s} \leq 0.0128 \\ -3.440\left(\ln \left(1-\phi_{s}\right)\right)+0.0110, \phi_{s} \geq 0.0128\end{array}\right.$

Table 3 - continued 


\section{3-D Filtered particle phase pressure:}

$$
\frac{\overline{p_{s, \text { filtered }}}}{\rho_{s} \mathrm{v}_{\mathrm{t}}^{2}}=\left\{\begin{array}{l}
\frac{\overline{p_{s, \text { kinetic }}}}{\rho_{s} \mathrm{v}_{\mathrm{t}}^{2}}+\text { Factor }_{p s} \phi_{s}\left(1-4.35 \phi_{s}+10 \phi_{s}^{2}-14.5 \phi_{s}^{3}+7.9 \phi_{s}^{4}\right), \phi_{s} \leq \phi_{s, \text { critical } 1} \\
\frac{p_{s, \text { kinetic }}}{\rho_{s} \mathrm{v}_{\mathrm{t}}^{2}}, \phi_{s}>\phi_{s, \text { rritical } 1}
\end{array}\right.
$$

where $\phi_{s, \text { critical_1 } 1}$ is defined as:

$$
\begin{aligned}
& \text { Factor }_{p s} \phi_{s}\left(1-4.35 \phi_{s}+10 \phi_{s}^{2}-14.5 \phi_{s}^{3}+7.9 \phi_{s}^{4}\right)=0 \text { at } \phi_{s}=\phi_{s, \text { critical_1 }} \\
& \text { Factor }_{p s}=\left\{\begin{array}{l}
0.491 F r_{f}^{-2}+0.150 F r_{f}^{-1}, F r_{f}^{-1}<1.29 \\
0.882 F r_{f}^{-0.68}-0.0101, F r_{f}^{-1}>1.29
\end{array}\right.
\end{aligned}
$$

The kinetic model term can be taken care of in two ways:

1. Also solve the granular energy equation

2. Simply add the curve fit for the kinetic particle phase pressure.

$$
\overline{\frac{p_{s, \text { kinetic }}}{\rho_{\mathrm{s}} \mathrm{v}_{\mathrm{t}}^{2}}}=\left\{\begin{array}{l}
-20.2 \phi_{\mathrm{s}}^{2}+0.611 \phi_{\mathrm{s}}, \phi_{\mathrm{s}} \leq 0.0140 \\
2.33 \phi_{\mathrm{s}}^{4}-2.48 \phi_{\mathrm{s}}^{3}+0.812 \phi_{\mathrm{s}}^{2}-0.0853 \phi_{\mathrm{s}}+0.00565,0.0140<\phi_{\mathrm{s}} \leq 0.353 \\
-0.00341 \phi_{\mathrm{s}}^{2}-0.0217 \phi_{\mathrm{s}}+0.0119,0.353<\phi_{\mathrm{s}} \leq 0.501 \\
1.41 \times 10^{-18} \exp \left(65.1 \phi_{\mathrm{s}}\right), 0.501<\phi_{\mathrm{s}} \leq 0.546 \\
2.33 \phi_{s}^{4}-2.48 \phi_{s}^{3}+0.812 \phi_{s}^{2}-0.0853 \phi_{s}+0.00500, \phi_{\mathrm{s}}>0.546
\end{array}\right.
$$

\section{3-D Filtered particle phase (shear) viscosity:}

$$
\frac{\overline{\mu_{s, \text { filtered }} g}}{\rho_{s} \mathrm{v}_{\mathrm{t}}^{3}}=\left\{\begin{array}{l}
\frac{\overline{\mu_{s, \text { kinetic }} g}}{\rho_{s} \mathrm{v}_{\mathrm{t}}^{3}}+\text { Factor }_{\mu s} \phi_{s}\left(1-4 \phi_{s}+27 \phi_{s}^{2}-72 \phi_{s}^{3}+54.8 \phi_{s}^{4}\right), \phi_{s} \leq \phi_{s, \text { critical_2 }} \\
\frac{\mu_{s, \text { kinetic }} g}{\rho_{s} \mathrm{v}_{\mathrm{t}}^{3}}, \phi_{s}>\phi_{s, \text { critical_2 }}
\end{array}\right.
$$

where $\phi_{s, \text { critical_2 }}$ is defined as:

Factor $_{\mu s} \phi_{s}\left(1-4 \phi_{s}+27 \phi_{s}^{2}-72 \phi_{s}^{3}+54.8 \phi_{s}^{4}\right)=0$ at $\phi_{s}=\phi_{s, \text { critical_2 }}$

Table 3 - continued 
Factor $_{\mu s}=\left\{\begin{array}{l}0.129 F r_{f}^{-2}+0.0191 F r_{f}^{-1}, F r_{f}^{-1}<1.38 \\ 0.197 F r_{f}^{-1.05}-0.00411, F r_{f}^{-1} \geq 1.38\end{array}\right.$

The kinetic model term in these models can be taken care of in two ways:

1. Also solve the granular energy equation

2. Simply add the curve fit for the kinetic particle phase viscosity

$\frac{\overline{\mu_{s, k i n e t i c}} g}{\rho_{\mathrm{s}} \mathrm{v}_{\mathrm{t}}^{3}}=7.87 \phi_{\mathrm{s}}^{7}-3.05 \phi_{\mathrm{s}}^{6}-7.24 \phi_{\mathrm{s}}^{5}+6.83 \phi_{\mathrm{s}}^{4}-2.44 \phi_{\mathrm{s}}^{3}+0.428 \phi_{\mathrm{s}}^{2}-0.0344 \phi_{\mathrm{s}}+0.00140$

Table 4: 3-D wall corrections to the filtered drag coefficient, particle phase pressure and viscosity 
Dimensionless distance from the wall of a riser with a circular cross-section (for cylindrical coordinates): $r_{d}=g\left(\frac{D_{\text {riser }}}{2}-r\right) / \mathrm{v}_{t}^{2}$

\section{Filtered drag coefficient:}

$\overline{\beta_{\text {filtered, scaled }}}=\frac{\overline{\beta_{\text {filtered }}}\left(\phi_{s}, r_{d}\right)}{\overline{\beta_{\text {filtered , core }}}\left(\phi_{s}\right)}=\frac{1}{1+4.5 \exp \left(-1.75 r_{d}\right)}, 0 \leq r_{d} \leq \frac{g D_{\text {riser }}}{2 \mathrm{v}_{t}^{2}}$

$\overline{\beta_{\text {filtered,core }}}\left(\phi_{s}\right)=\overline{\beta_{\text {filtered,periodic }}}\left(\phi_{s}\right)$

\section{Filtered particle phase pressure:}

$$
\overline{p_{s, \text { filtered,scaled }}}=\frac{\overline{p_{s, \text { filtered }}}\left(\phi_{s}, r_{d}\right)}{\overline{p_{s, \text { filtered , core }}\left(\phi_{s}\right)}}=\frac{1}{1+4.5 \exp \left(-1.75 r_{d}\right)}, 0 \leq r_{d} \leq \frac{g D_{\text {riser }}}{2 \mathrm{v}_{t}^{2}}
$$

$\overline{p_{s, \text { filtered,core }}}\left(\phi_{s}\right)=\overline{p_{\text {s, filtered,periodic }}}\left(\phi_{s}\right)$

\section{Filtered particle phase shear viscosity:}

$$
\begin{aligned}
& \overline{\mu_{s, \text { filtered,scaled }}}=\frac{\overline{\mu_{s, \text { filtered }}}\left(\phi_{s}, r_{d}\right)}{\overline{\mu_{s, \text { filtered,core }}\left(\phi_{s}\right)}}=\frac{1}{1+4.5 \exp \left(-1.75 r_{d}\right)}, 0 \leq r_{d} \leq \frac{g D_{\text {riser }}}{2 \mathrm{v}_{t}^{2}} \\
& \overline{\mu_{s, \text { filtered,core }}}\left(\phi_{s}\right)=\overline{\mu_{s, \text { filtered,periodic }}}\left(\phi_{s}\right)
\end{aligned}
$$

\section{Figures:}




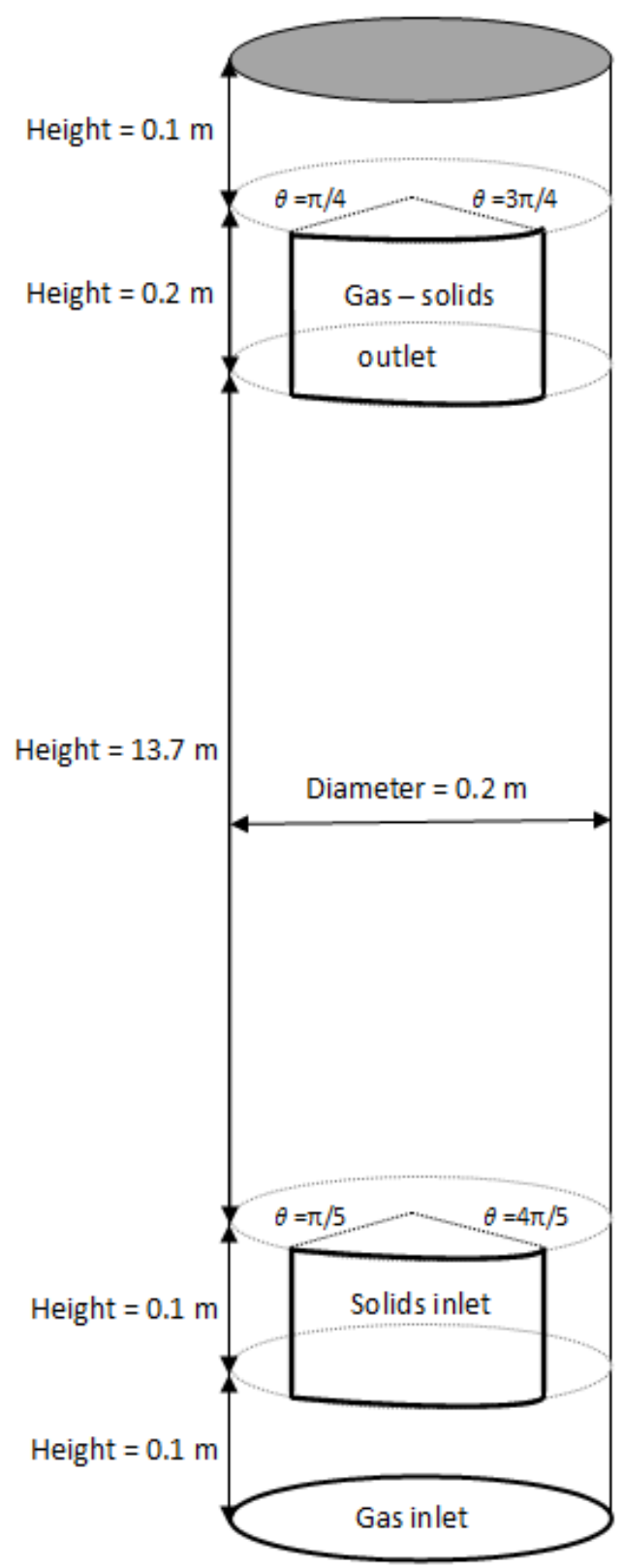

Figure 1: The 3-D computational domain used for simulations to capture the experimental data obtained from the PSRI riser. This set-up and the schematic were adapted from Benyahia ${ }^{49}$. In this set-up, the gas enters the domain uniformly at the bottom whereas the particles enter from the inlet located on the side wall. The gas and particles are allowed to leave through exit region located on the side wall $(0.1 \mathrm{~m}$ below the splash plate located over entire top area of the riser). The details of the exit and outlet geometries and the simulation conditions are given in the main text. The physical conditions corresponding to this simulation is listed in Table 1. 


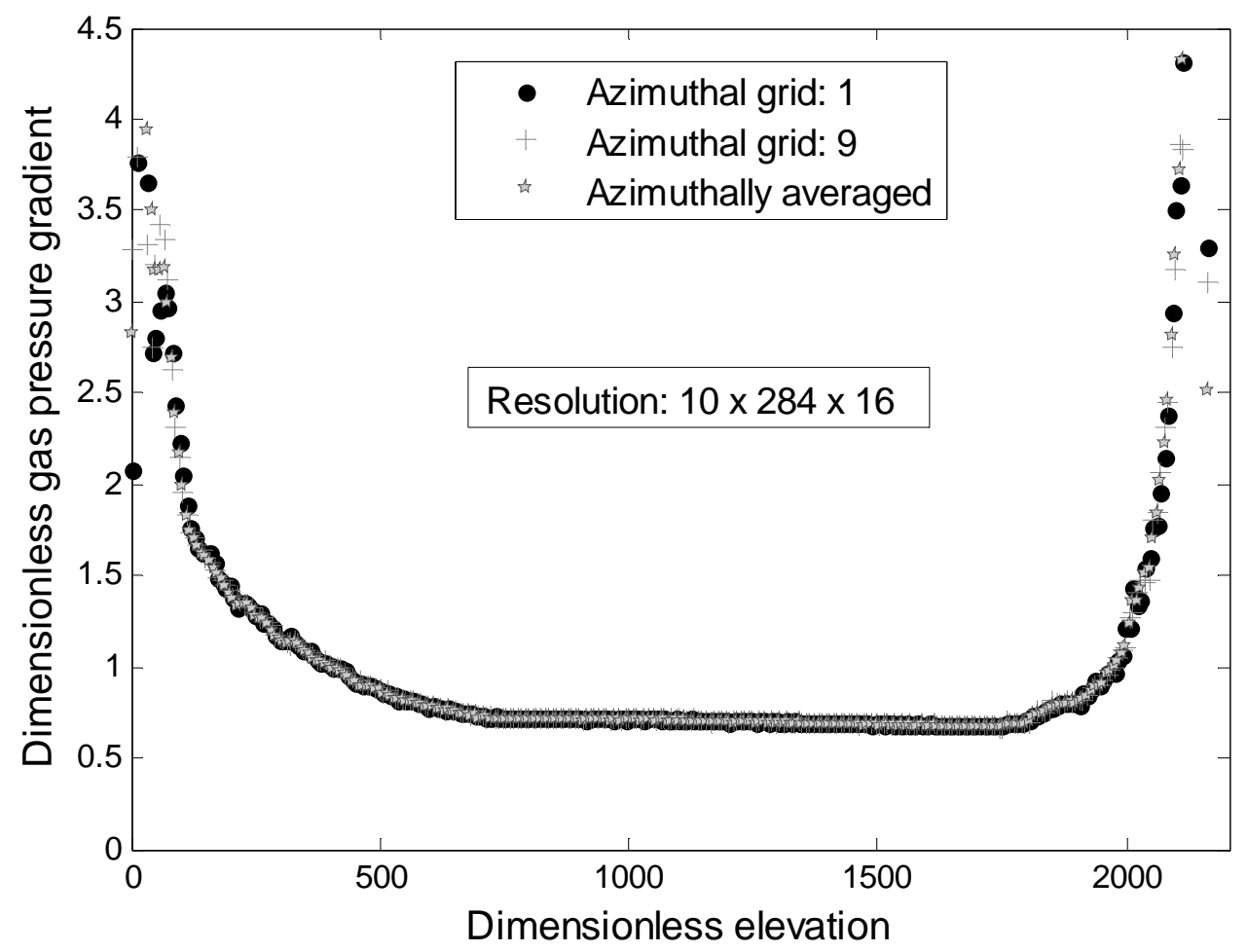

Figure 2: The variation of the dimensionless time-averaged gas pressure gradient along the height of the 3-D riser with azimuthal location (grid). An azimuthally averaged profile is also included. The filtered model used in this simulation was extracted with a filter length of 4.112 dimensionless units. Free-slip boundary condition was imposed for both phases at all walls. Note that azimuthal grids 1 and 9 are $180^{\circ}$ apart. Resolution: $10 \times 284 \times 16$. For these studies, we set the gas (actual) velocity to $5.2 \mathrm{~m} / \mathrm{s}$ at the inlet. 

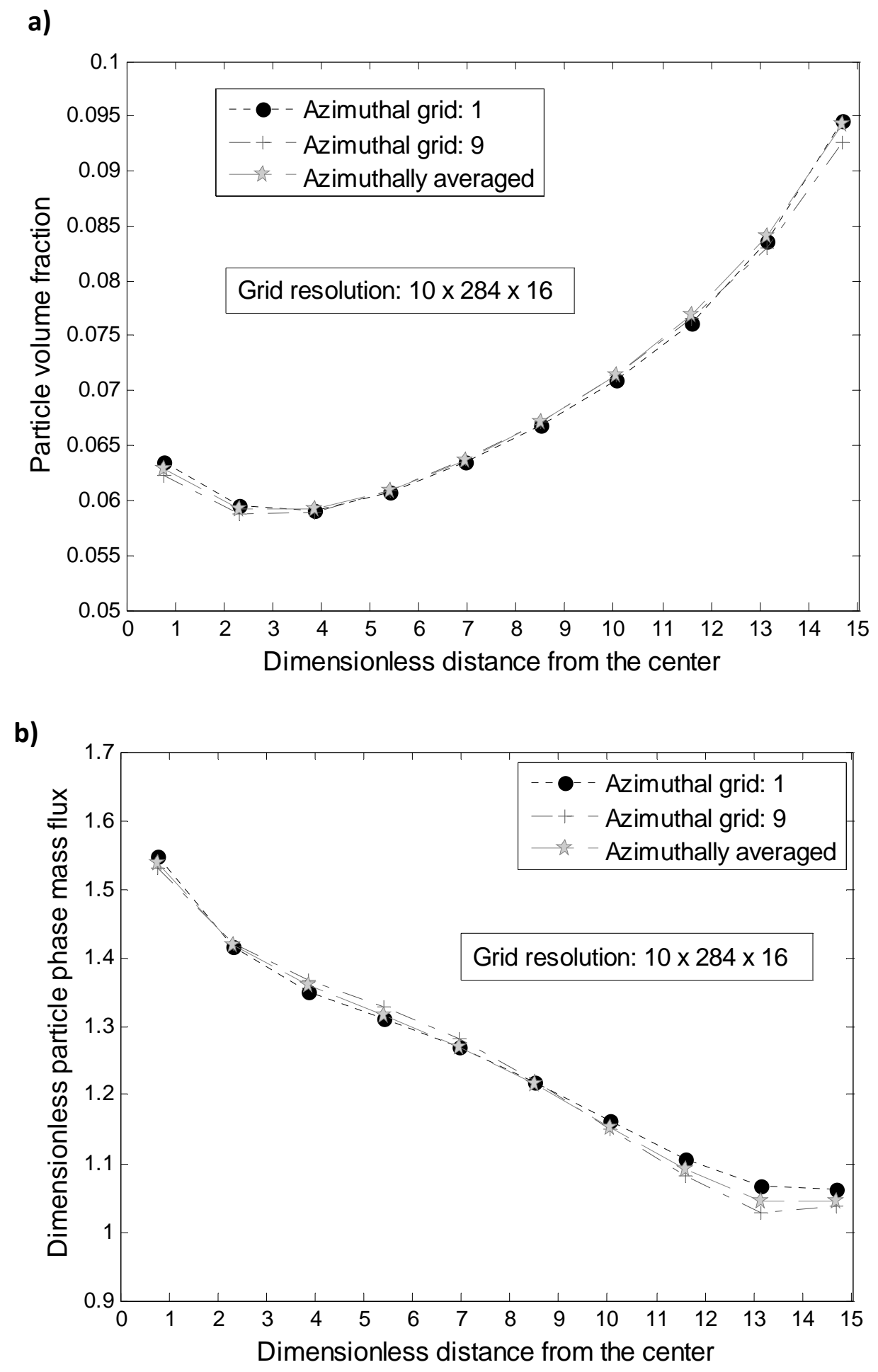

Figure 3: Dimensionless time-averaged profiles of (a) particle volume fraction and (b) axial particle mass flux at an elevation of 602.9 dimensionless units $(=3.9 \mathrm{~m})$ above the particles inlet were obtained from two azimuthal locations shown in the figure legend ( $180^{\circ}$ apart). Azimuthally averaged profiles are also included. Everything else is the same as in Figure 2. 


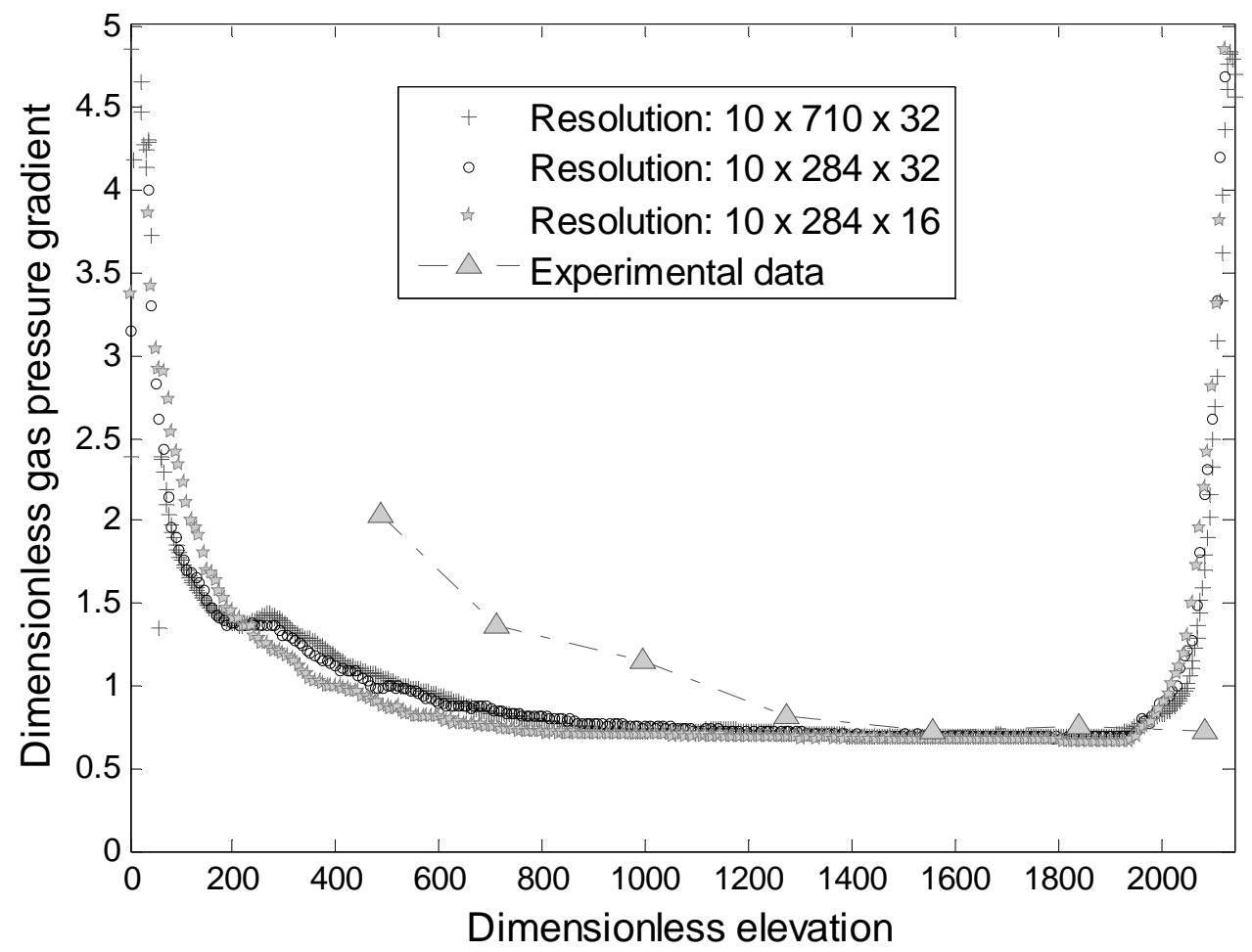

Figure 4: The effect of grid resolution on the dimensionless time-averaged gas pressure gradient along the height of the 3-D riser. Azimuthally averaged profiles are used. The experimental data are also included for comparison. The filtered model used in this simulation was extracted with a filter length of 4.112 dimensionless units. Free-slip boundary condition was imposed for both phases at all walls. For these studies, we set the gas (actual) velocity to $5.2 \mathrm{~m} / \mathrm{s}$ at the inlet. 

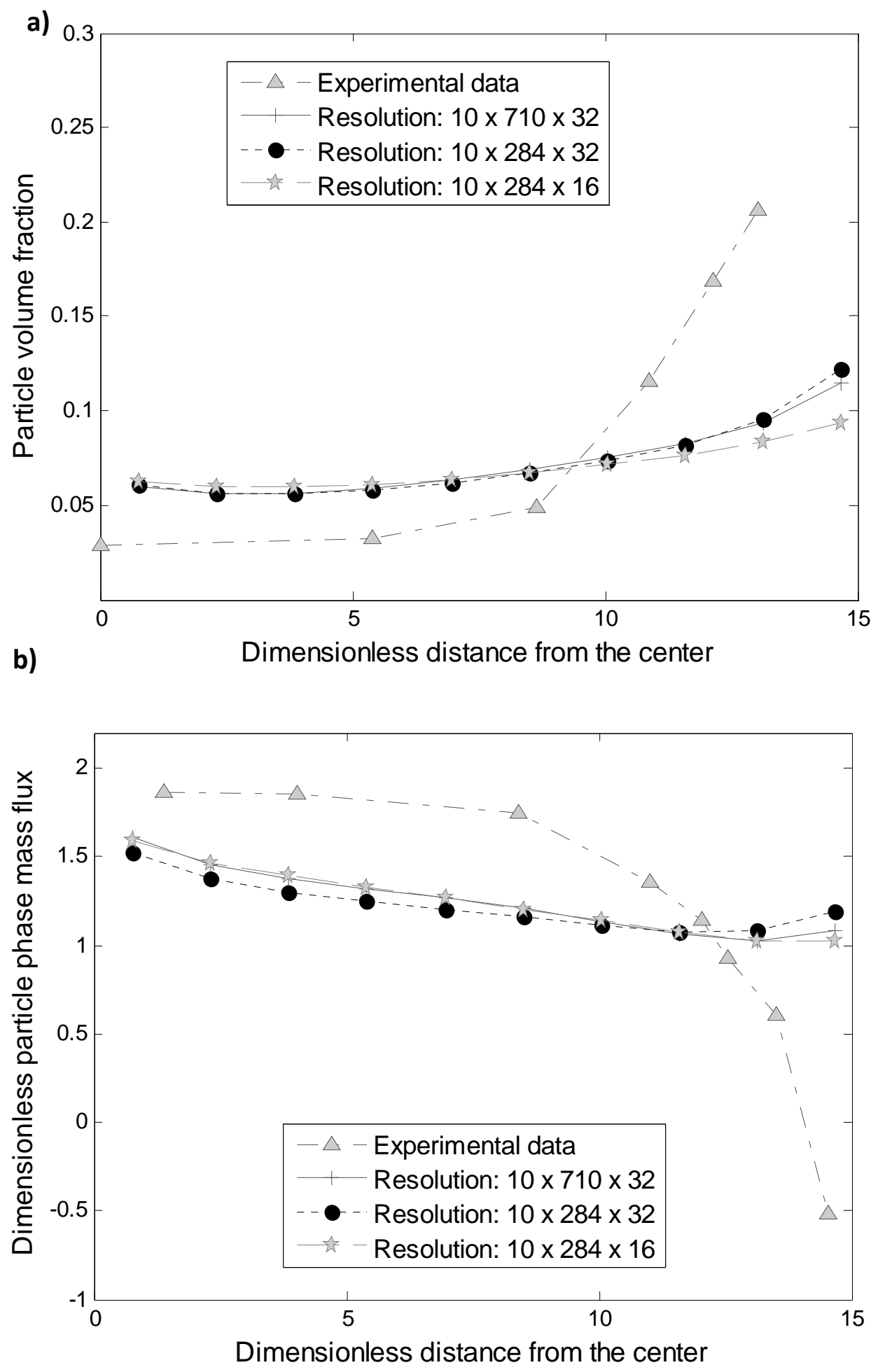

Figure 5: The effect of grid resolution on the dimensionless time-averaged profiles of (a) particle volume fraction and (b) axial particle mass flux at an elevation of 602.9 dimensionless units $(=3.9 \mathrm{~m})$ above the solids inlet. Azimuthally averaged profiles are used. The experimental data are also included for comparison. Everything else is the same as in Figure 4. 


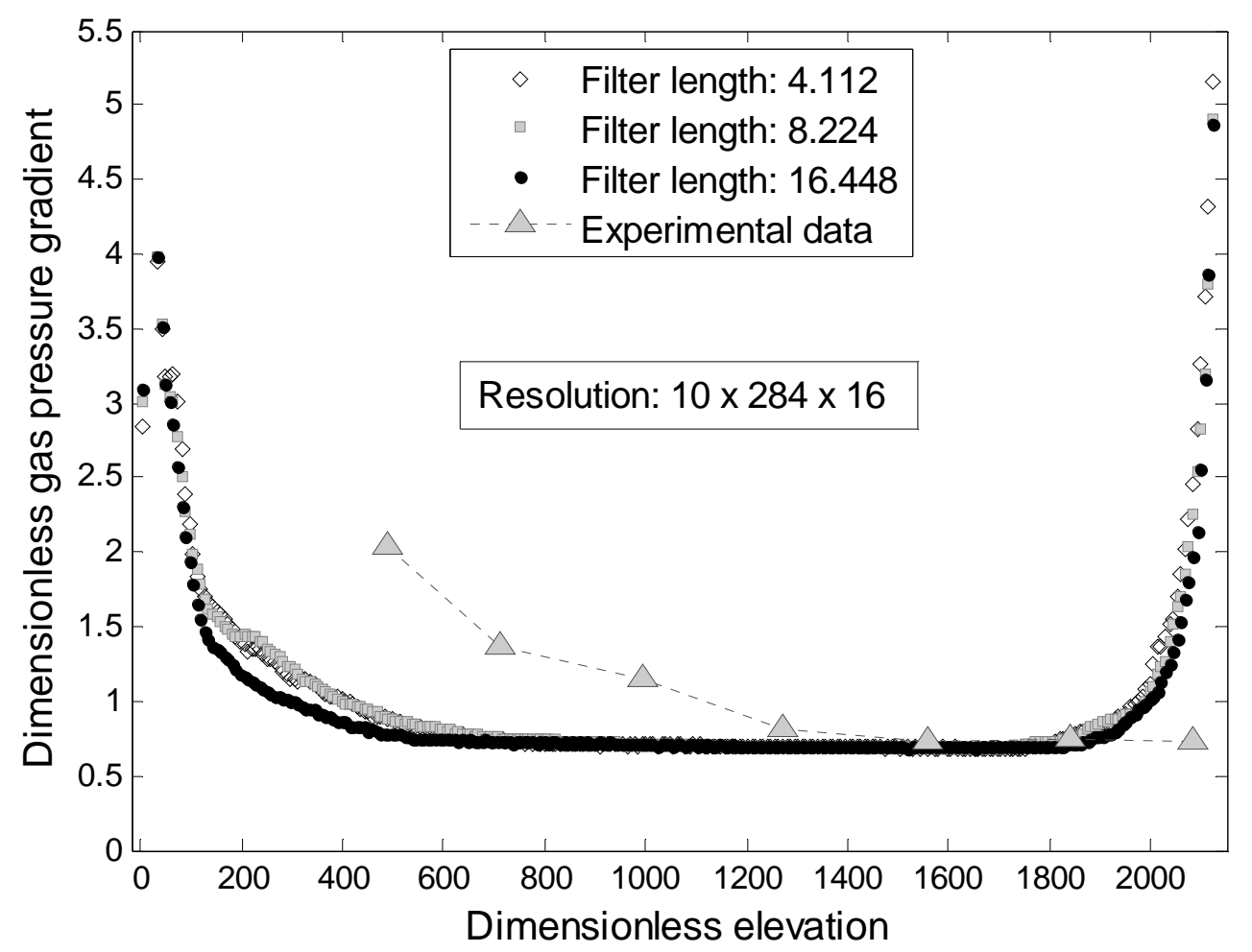

Figure 6: The effect of the filter size (used to extract the filtered models) on the dimensionless timeaveraged gas pressure gradient along the height of the 3-D riser. Free-slip boundary condition was imposed for both phases at all walls. Resolution: 10 x 284 x 16. For these studies, we set the gas (actual) velocity to $5.2 \mathrm{~m} / \mathrm{s}$ at the inlet. The experimental data are also included for comparison. 
a)
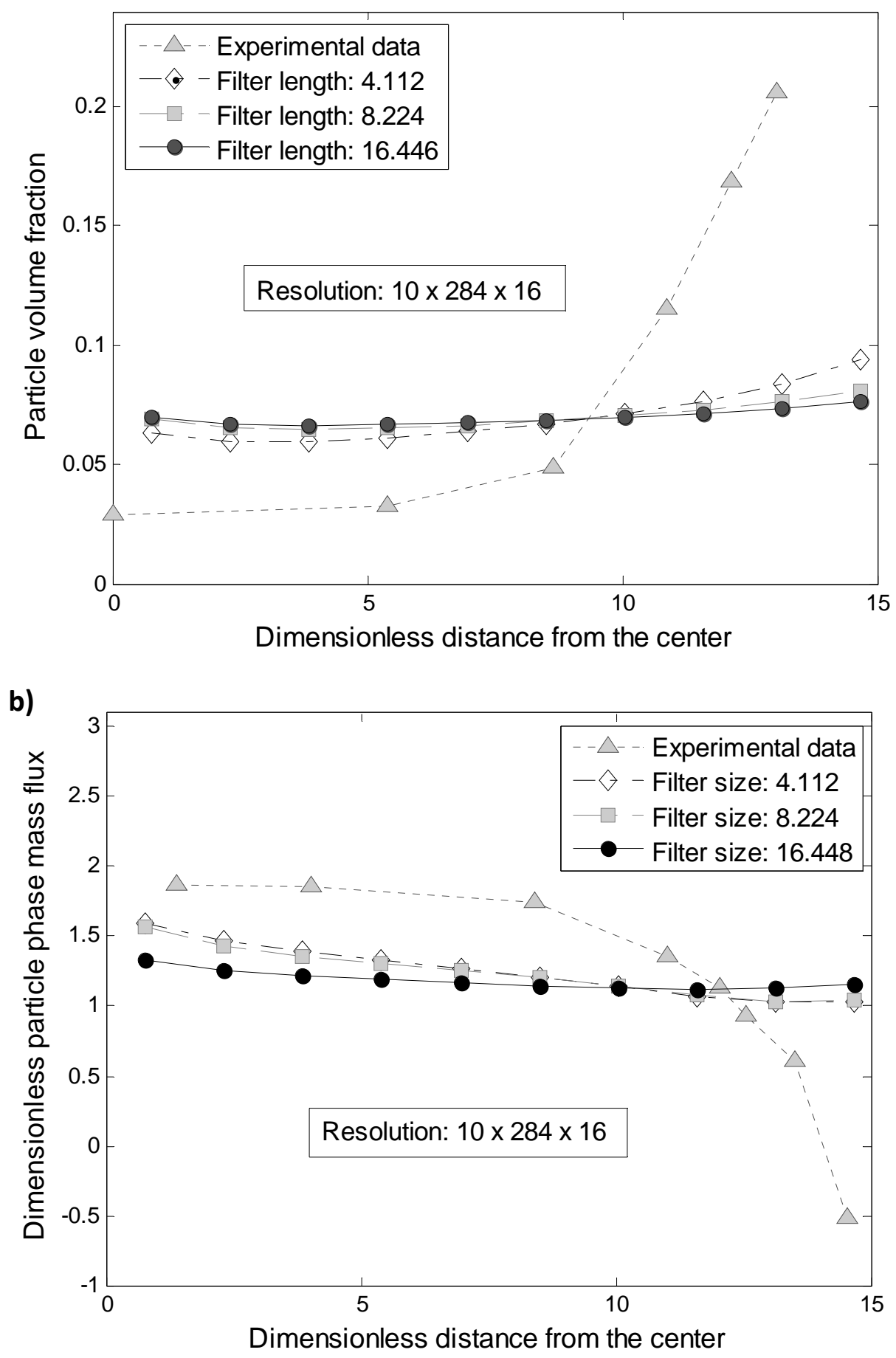

Figure 7: The effect of the filter size (used to extract the filtered models) on the dimensionless timeaveraged profiles of (a) particle volume fraction and (b) axial particle mass flux at an elevation of 602.9 dimensionless units $(=3.9 \mathrm{~m})$ above the solids inlet. Wall corrections were included in the filtered models given in the figure legend. The experimental data are also included for comparison. Everything else is the same as in Figure 6. 


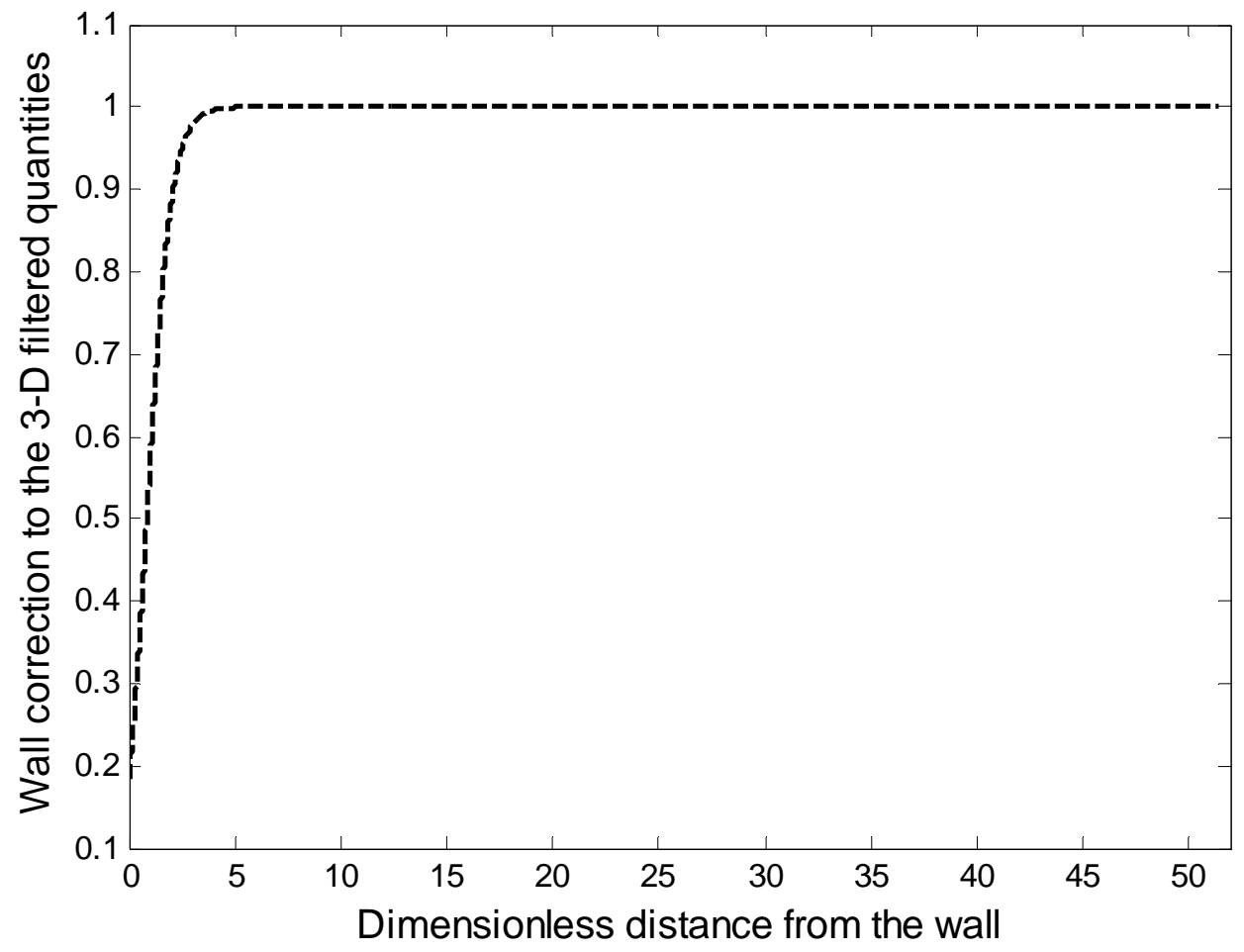

Figure 8: The wall corrections to the constitutive models for the filtered drag coefficient, particle phase pressure and viscosity were extracted from a 3-D kinetic theory based simulation of a riser with a square cross-section $(14.6 \mathrm{~cm} \times 914 \mathrm{~cm} \times 14.6 \mathrm{~cm}$ ) and adapted for a riser with a circular cross-section (e.g. the PSRI riser used in our studies). We have seen that the wall corrections for the filtered drag, particle phase pressure and viscosity are similar. Therefore, we have used the same correction for all three. See Table 4 for the correlations capturing the wall correction shown in this figure. 


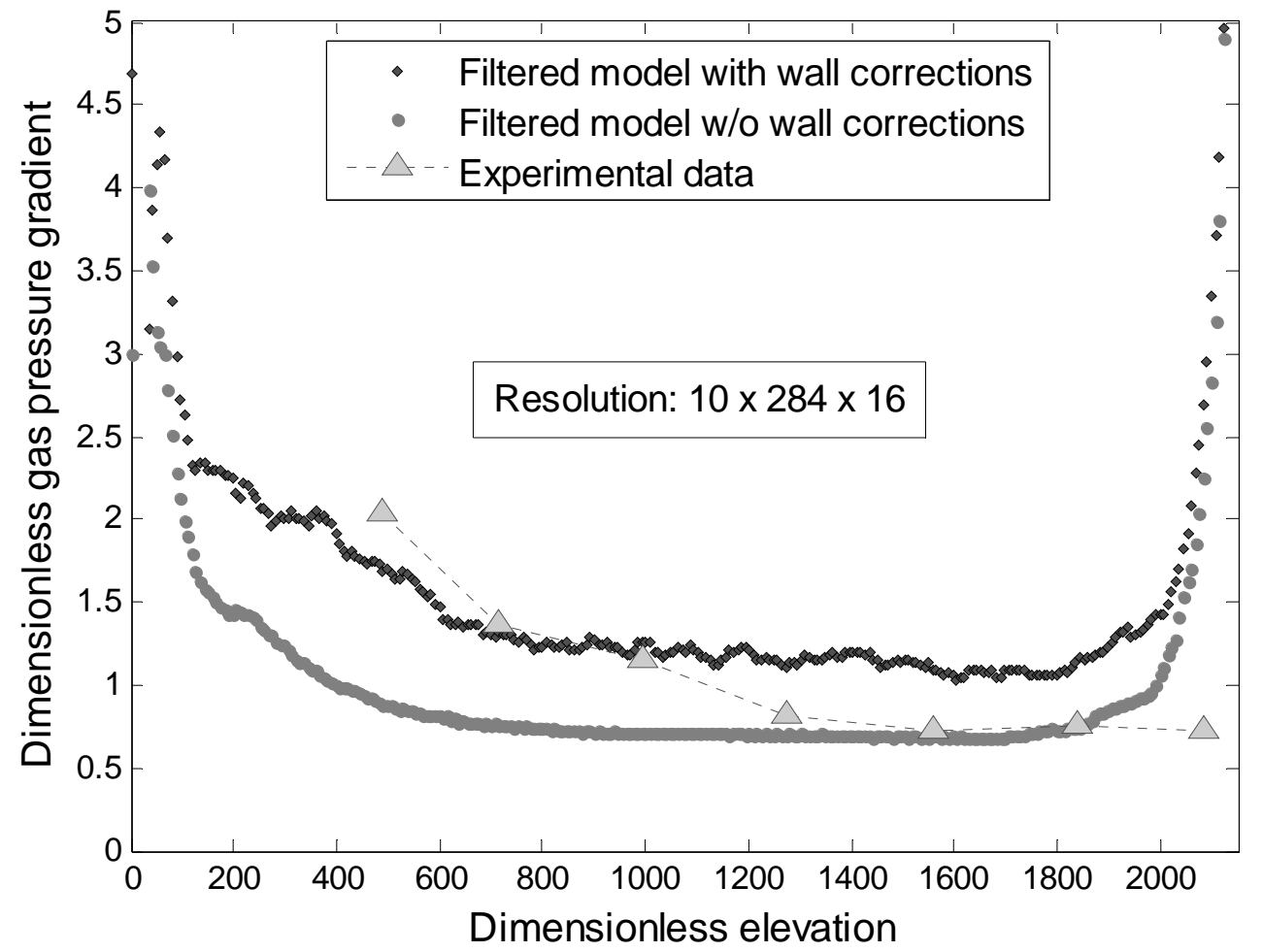

Figure 9: Dimensionless time-averaged gas pressure gradient profiles plotted along the height of the 3-D riser. Numerical predictions obtained with the filtered model closures with and without wall corrections (extracted with a filter length of 8.224 dimensionless units) are compared with the experimental data. Resolution: $10 \times 284 \times 16$. For these studies, we set the gas (actual) velocity to $5.2 \mathrm{~m} / \mathrm{s}$ at the inlet. 
a)

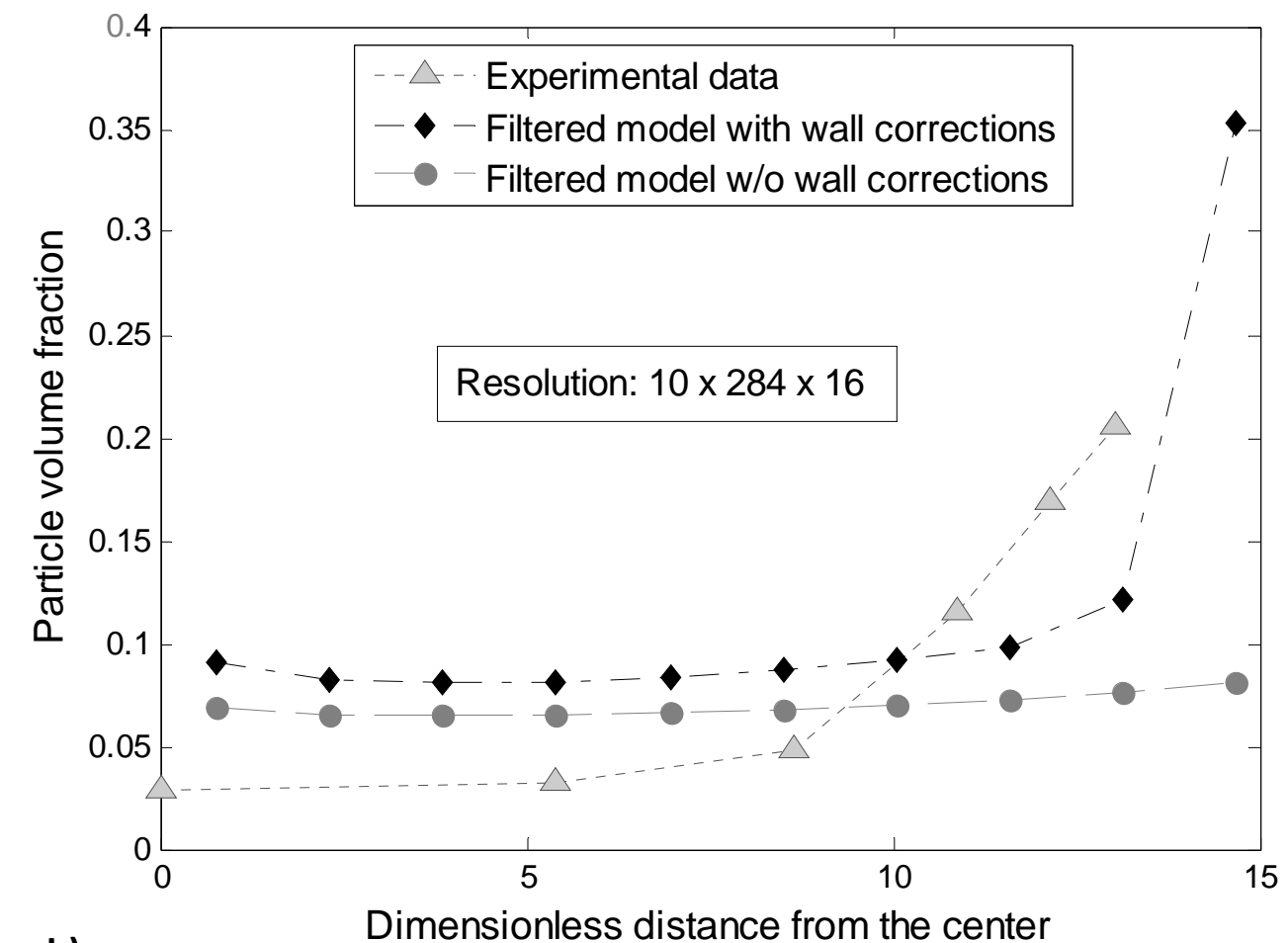

b)

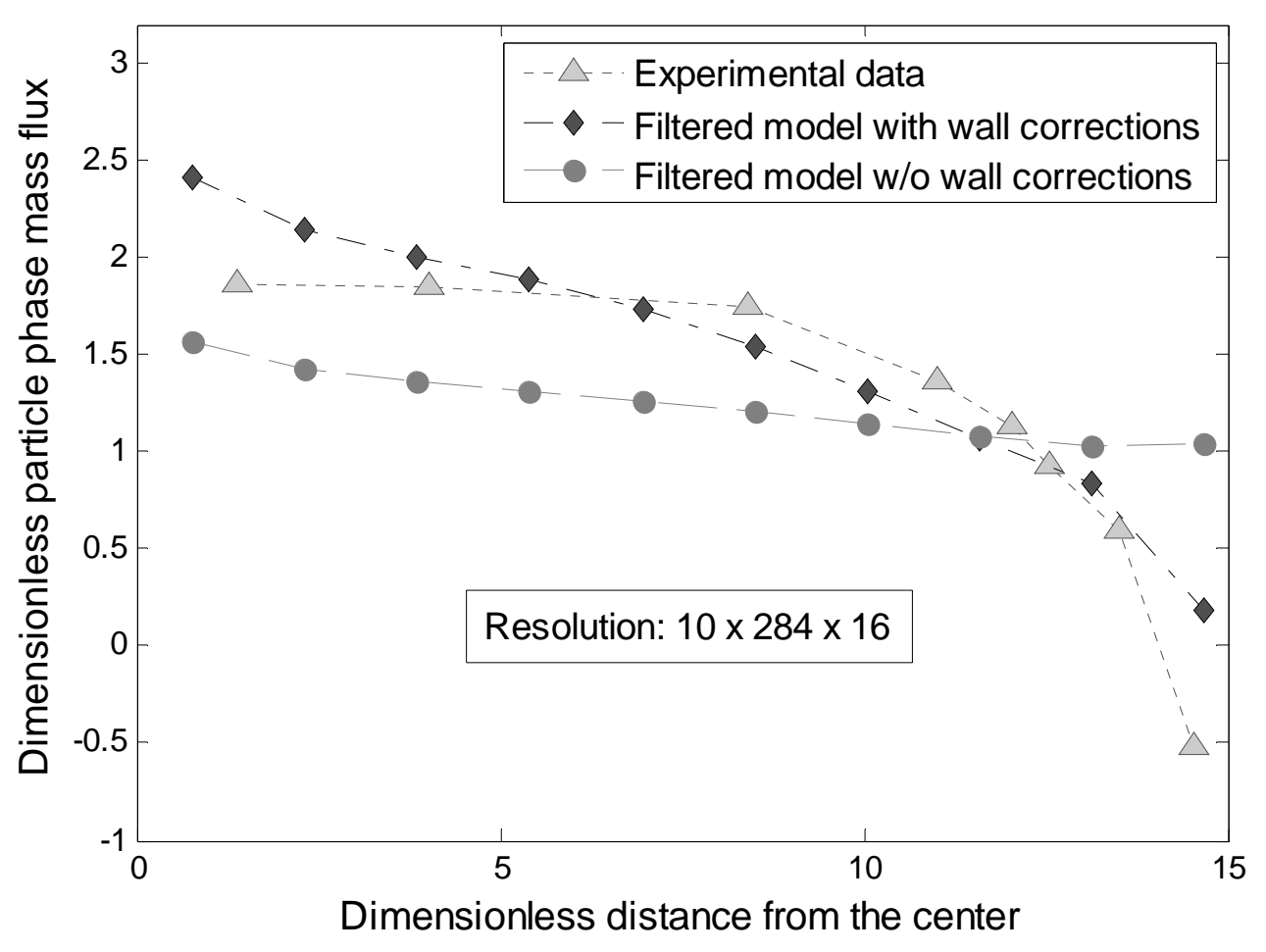




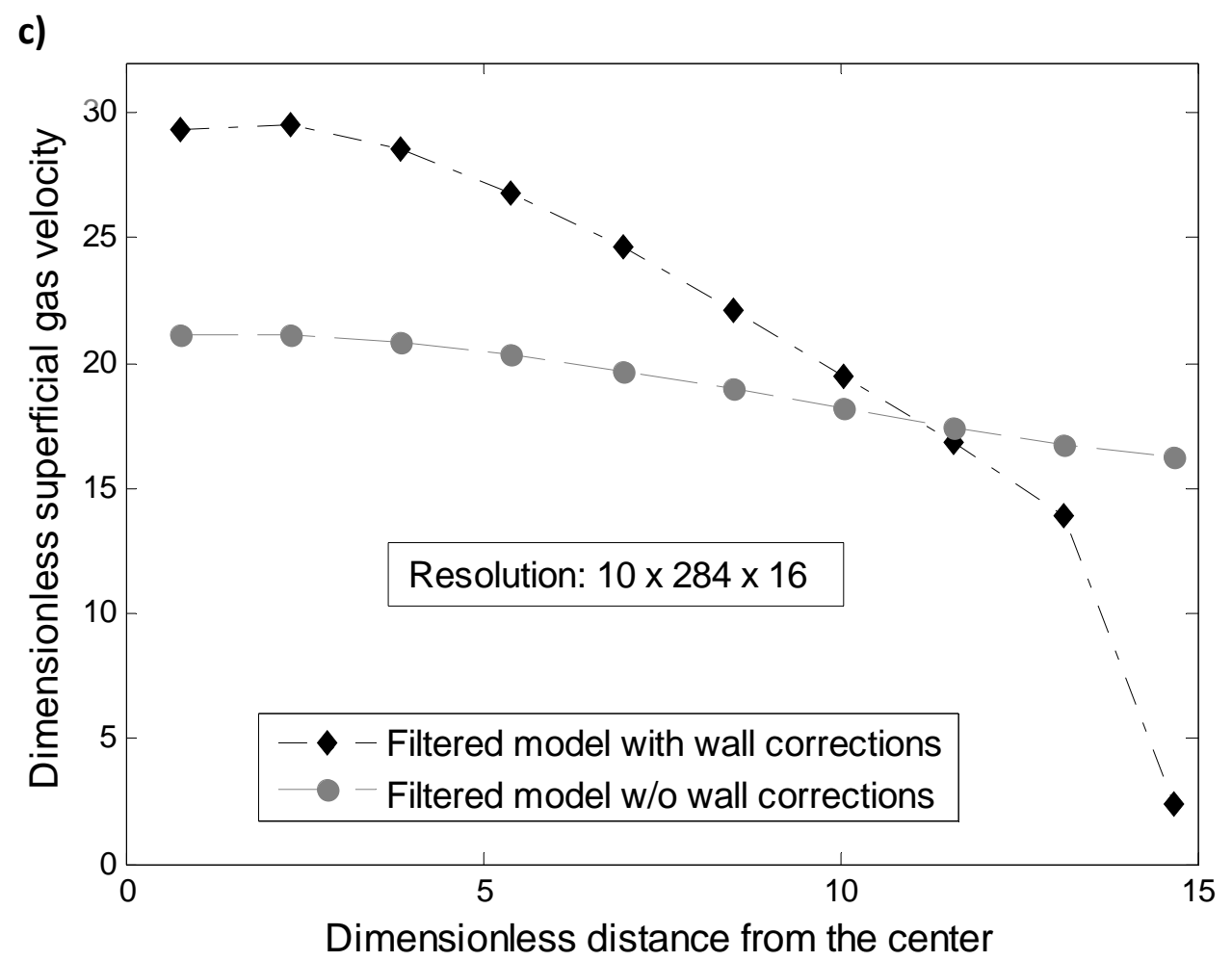

Figure 10: Dimensionless time-averaged radial profiles of (a) particle volume fraction, (b) axial particle mass flux and (c) axial superficial gas velocity at an elevation of 602.9 dimensionless units $(=3.9 \mathrm{~m})$ above the solids inlet obtained with the filtered closures (8.224) with and w/o wall corrections. The experimental data are also shown for comparison. Everything else is the same as in Figure 9. 


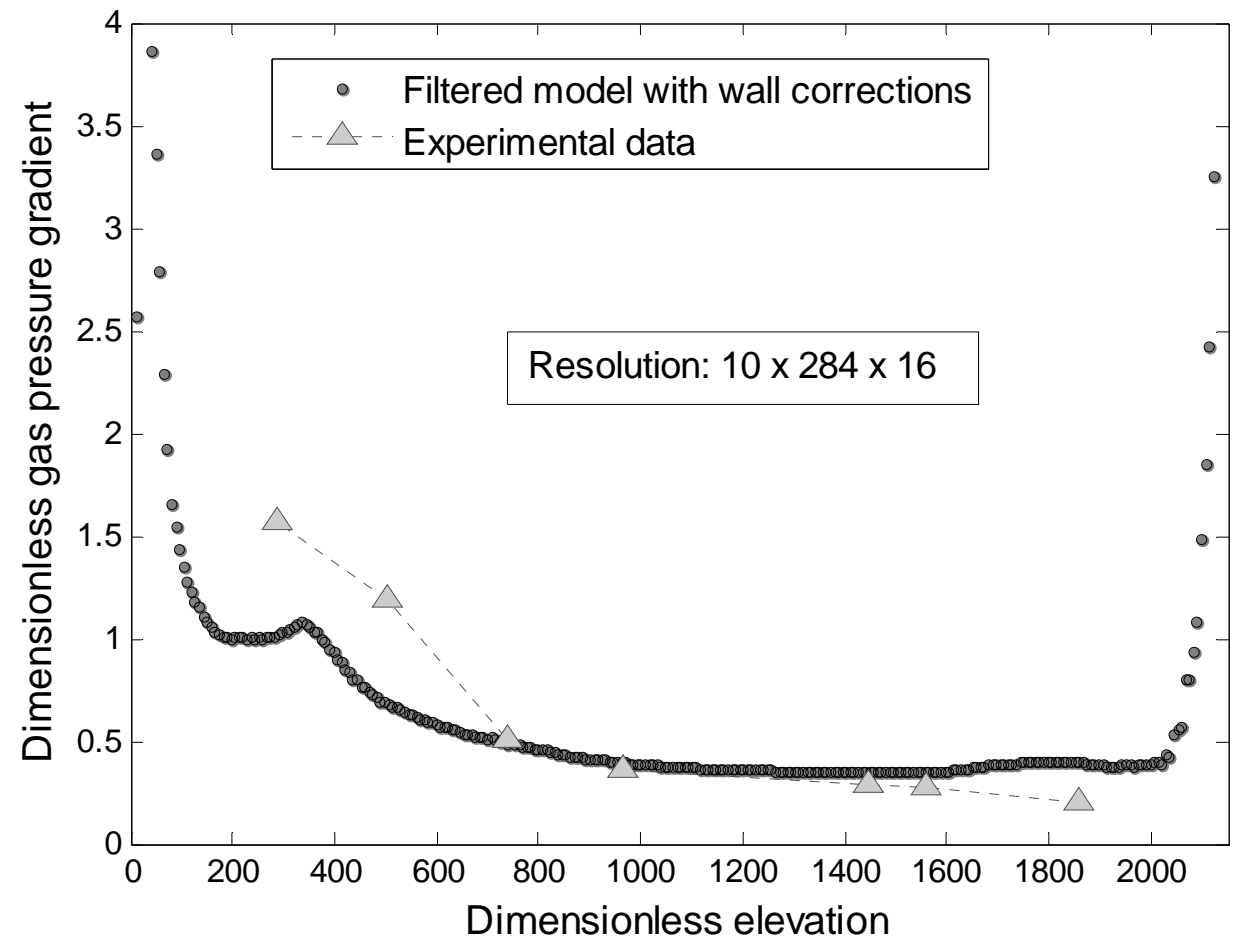

Figure 11: Dimensionless time-averaged gas pressure gradient profiles plotted along the height of the 3D riser. Numerical predictions obtained with the filtered model closures with wall corrections (extracted with a filter size of 8.224 dimensionless units) are compared with the experimental data. Resolution: $10 \mathrm{x}$ $284 \times 16$. For these studies, we set the gas (actual) velocity to $11 \mathrm{~m} / \mathrm{s}$ at the inlet. 


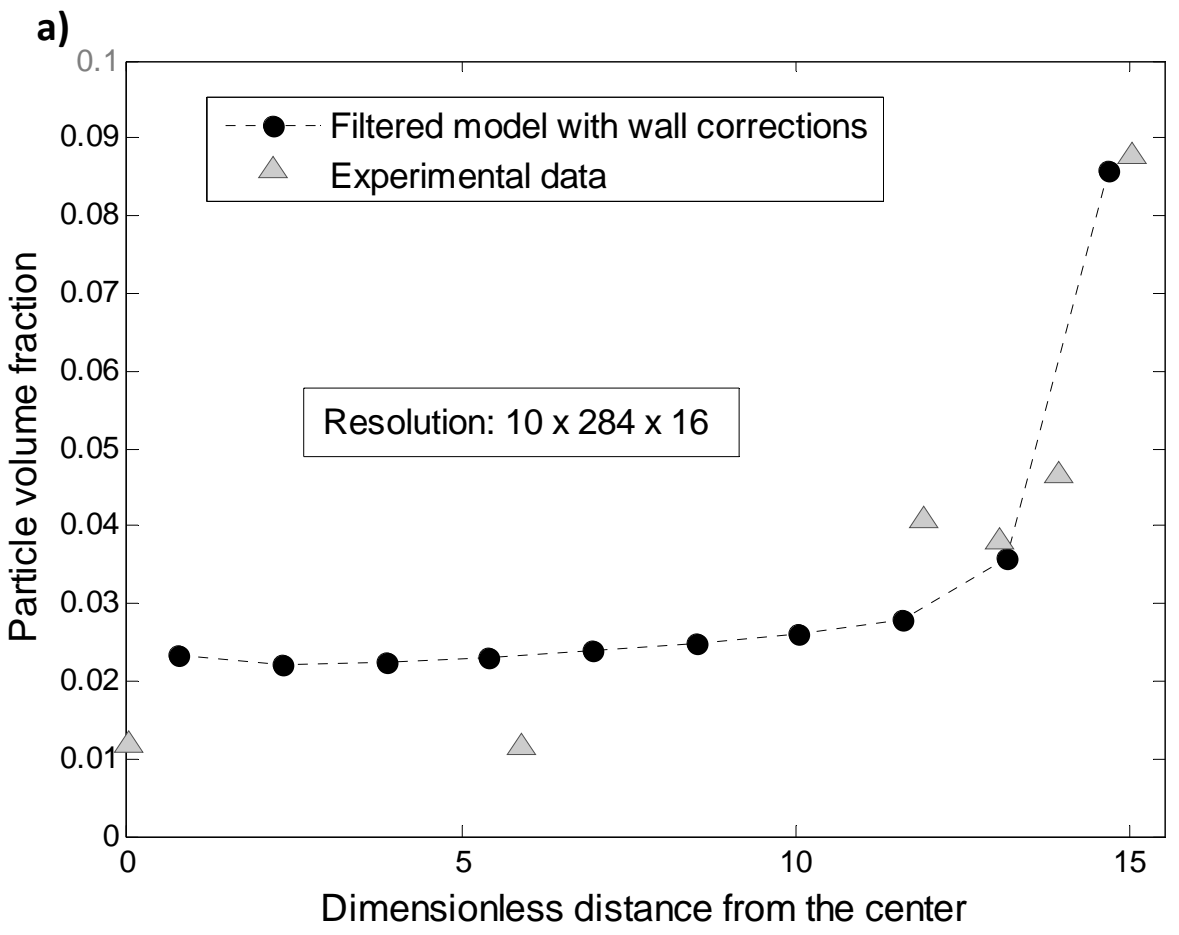

b)

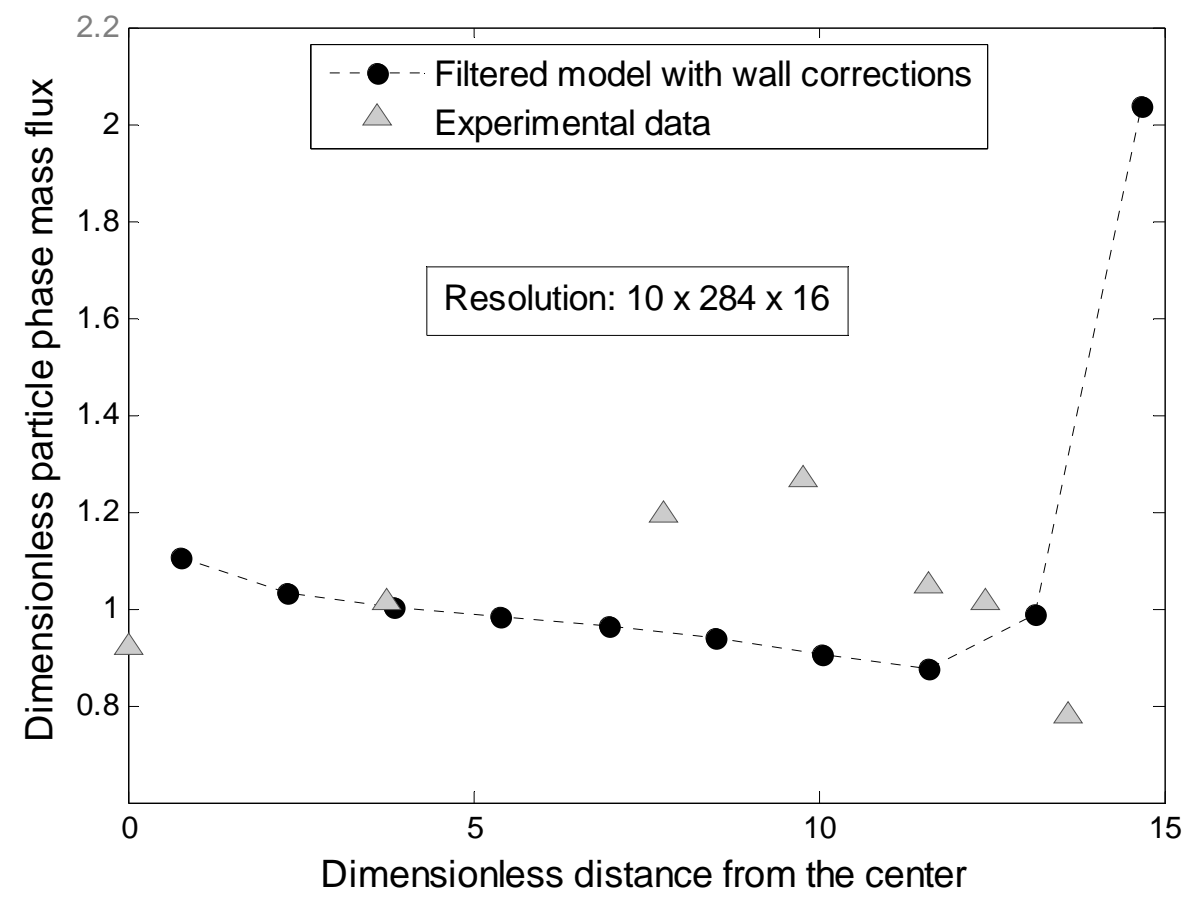

Figure 12: Dimensionless time-averaged profiles of (a) particle volume fraction and (b) axial particle mass flux at an elevation of 602.9 dimensionless units $(=3.9 \mathrm{~m})$ above the solids inlet obtained with the filtered closures (8.224) with and w/o wall corrections. The experimental data are also shown for comparison. Everything else is the same as in Figure 11. 
APPENDIX A 


\section{Filtered two-fluid models for fluidized gas-particle suspensions}

by

Yesim Igci ${ }^{1}$, Arthur T. Andrews IV ${ }^{1}$, Sreekanth Pannala ${ }^{2}$, Thomas O’Brien ${ }^{3}$, and Sankaran Sundaresan ${ }^{1, *}$

1. Department of Chemical Engineering, Princeton University, Princeton, NJ 08544

2. Oak Ridge National Laboratory, Oak Ridge, TN 37831

3. National Energy Technology Laboratory, Morgantown, WV 26507

*. Corresponding author: (609) 258-4583; sundar@princeton.edu

Published online March 28, 2008 in Wiley InterScience (www.interscience.wiley.com)

(AIChE J, 54: 1431-1448, 2008) 


\section{Abstract}

Starting from a kinetic theory based two-fluid model for gas-particle flows, we first construct filtered two-fluid model equations that average over small scale inhomogeneities which we do not wish to resolve in numerical simulations. We then outline a procedure to extract constitutive models for these filtered two-fluid models through highly resolved simulations of the kinetic theory based model equations in periodic domains. We demonstrate through two- and three- dimensional simulations that the closure relations for the filtered two-fluid models manifest a definite and systematic dependence on the filter size, which is examined in detail. In order to demonstrate that filtering does indeed remove small scale structures that are afforded by the microscopic two-fluid models, we performed 1D linear stability analyses of the filtered two-fluid model equations. These analyses show that filtering is indeed erasing the fine structure and only presenting coarser structures 


\section{Introduction}

Chemical reactors that take the form of fluidized beds and circulating fluidized beds are widely used in energy-related and chemical process industries. ${ }^{1}$ Gas-particle flows in these devices are inherently unstable; they manifest fluctuations over a wide range of length and time scales. Analysis of the performance of large scale fluidized bed processes through computational simulations of hydrodynamics and energy/species transport is becoming increasingly common. In the present study, we are concerned with the development of hydrodynamic models which are useful for simulation of gas-particle flows in large scale fluidized processes.

The number of particles present in most gas-particle flow systems is large, rendering detailed description of the motion of all the particles and fluid elements impractical. Hence, two-

fluid model equations ${ }^{2-4}$ are commonly employed to probe the flow characteristics, and species and energy transport. In this approach, the gas and particle phases are treated as inter-penetrating continua, and locally-averaged quantities such as the volume fractions, velocities, species concentrations and temperatures of gas and particle phases appear as dependent field variables. The averaging process leading to two-fluid model equations erases the details of flow at the level of individual particles; but their consequences appear in the averaged equations through terms for which one must develop constitutive relations. For example, in the momentum balance equations, constitutive relations are needed for the gas-particle interaction force and the effective stresses in the gas and particle phases.

The general form of the two-fluid model equations is fairly standard and this has permitted the development of numerical algorithms for solving them. For example, open-source packages such as $\operatorname{MFIX}^{4,5}$ and commercial software (e.g., Fluent $\left.{ }^{\circledR}\right)$ can readily be applied to 
perform transient integration (of the discretized forms) of the balance equations governing reactive and non-reactive multiphase flows. The results generated through such simulations are dependent on the postulated constitutive models, and a major focus of research over the past few decades has been on the improvement of these constitutive models.

Through a combination of experiments and computer simulations, constitutive relations have been developed in the literature for the fluid-particle interaction force and the effective stresses in the fluid and particle phases. In gas-particle systems, the interaction force is predominantly due to the drag force. An empirical drag law that bridges the results of Wen \& $\mathrm{Yu}^{6}$ for dilute systems and the Ergun ${ }^{7}$ approach for dense systems is widely used in simulation studies. ${ }^{2}$ In the past decade, $a b$ initio drag force models have also been developed via detailed simulations of fluid flow around assemblies of particles. ${ }^{8-14}$

The Stokes number associated with the particles in many gas-particle mixtures is sufficiently large that particle-particle and particle-wall collisions do occur; furthermore, when the particle volume fraction is below $\sim 0.5$, the particle-particle interactions occur largely through binary collisions. The particle phase stress in these systems is widely modeled through the kinetic theory of granular materials. ${ }^{2,15,16}$ This kinetic theory approach has also been extended to systems containing mixtures of different types of particles. ${ }^{2,17-20}$

It is important to keep in mind that all these closures are derived from data or analysis of nearly homogeneous systems. Henceforth, we will refer to the two-fluid model equations coupled with constitutive relations deduced from nearly homogeneous systems as the microscopic two-fluid model equations. For example, the kinetic theory based model equations described and simulated in most of the literature references fall in this category., ${ }^{2,16-31}$ 
A practical difficulty comes about when one tries to solve these microscopic two-fluid model equations for gas-particle flows. Gas-particle flows in fluidized beds and riser reactors are inherently unstable, and they manifest inhomogeneous structures over a wide range of length and time scales. There is a substantial body of literature where researchers have sought to capture these fluctuations through numerical simulation of microscopic two-fluid model equations. Indeed, two-fluid models for such flows reveal unstable modes whose length scale is as small as ten particle diameters. ${ }^{30,31}$ This can readily be ascertained by simple simulations, as illustrated in Figure 1. Transient simulations of a fluidized suspension of ambient air and typical Fluid Catalytic Cracking catalyst particles were performed (using MFIX $^{4,5}$ ) in a Cartesian, twodimensional (2-D), periodic domain at different grid resolutions; these simulations employed kinetic theory based (microscopic) two-fluid model equations (summarized in Table 1 and briefly discussed in $\$ 2$ below). The relevant parameter values can be found in Table 2 . The simulations revealed that an initially homogeneous suspension gave way to an inhomogeneous state with persistent fluctuations. Snapshots of the particle volume fraction fields obtained in simulations with different spatial grid resolution are shown in Figure 1. It is readily apparent that finer and finer structures are resolved as the spatial grid is refined. Statistical quantities obtained by averaging over the whole domain were found to depend on the grid resolution employed in the simulations and they became nearly grid-size independent only when grid sizes of the order of a few particle diameters were used (see Agrawal et $a .^{30}$ for further discussion). Thus, if one sets out to solve the microscopic two-fluid model equations for gas-particle flows, grid sizes of the order of a few particle diameters become necessary. Moreover, such fine spatial resolution reduces the numerical time steps required, further increasing the computational effort. For most devices of practical (commercial) interest, such extremely fine spatial grids and small time steps 
are unaffordable. ${ }^{32}$ Indeed, gas-particle flows in large fluidized beds and risers are often simulated by solving discretized versions of the two-fluid model equations over a coarse spatial grid. Such coarse grid simulations do not resolve the small-scale (i.e., sub-grid scale) spatial structures which, according to the microscopic two-fluid equations and experimental observation, do indeed exist. The effect of these unresolved structures on the structures resolved in coarse-grid simulations must be accounted for through appropriate modifications to the closures - for example, the effective drag coefficient in the coarse-grid simulations will be smaller than that in the original two-fluid model to reflect the tendency of the gas to flow more easily around the unresolved clusters ${ }^{30,31}$ than through a homogenous distribution of these particles. Qualitatively, this is equivalent to an effectively larger apparent size for the particles.

One can readily pursue this line of thought and examine the influence of these unresolved structures on the effective inter-phase transfer and dispersion coefficients which should be used in coarse-grid simulations. Inhomogeneous distribution of particles will promote bypassing of the gas around the particle-rich regions and this will necessarily decrease the effective interphase mass and energy transfer rates. Conversely, fluctuations associated with the small scale inhomogeneities will contribute to the dispersion of the particles and the gas, but this effect will be unaccounted for in the coarse-grid simulations of the microscopic two-fluid models.

Researchers have approached this problem of treating unresolved structures through various approximate schemes. O'Brien \& Syamla ${ }^{33}$, Boemer et al. $^{34}$ and Heynderickx et al. ${ }^{35}$ pointed out the need to correct the drag coefficient to account for the consequence of clustering, and proposed a correction for the very dilute limit. Some authors have used an apparent cluster size in an effective drag coefficient closure as a tuning parameter, ${ }^{36}$ others have deduced corrections to the drag coefficient using an Energy Minimization Multi-Scale approach. ${ }^{37}$ The 
concept of particle phase turbulence has also been explored to introduce the effect of the fluctuations associated with clusters and streamers on the particle phase stresses. ${ }^{38,39}$ However, a systematic approach that combines the influence of the unresolved structures on the drag coefficient and the stresses has not yet emerged. The effects of these unresolved structures on inter-phase transfer and dispersion coefficients remain unexplored.

Agrawal et al. ${ }^{30}$ showed that the effective drag law and the effective stresses, obtained by averaging (the results gathered in highly resolved simulations of a set of microscopic two-fluid model equations) over the whole (periodic) domain, were very different from those used in the microscopic two-fluid model and that they depended on size of the periodic domain. They also demonstrated that all the effects seen in the 2-D simulations persisted when simulations were repeated in three dimensions (3-D) and that both 2-D and 3-D simulations revealed the same qualitative trends. Andrews et al. ${ }^{31}$ performed many highly resolved simulations of fluidized gasparticle mixtures in a 2-D periodic domain whose total size coincided with that of the grid size in an anticipated large-scale riser flow simulation. Using these numerical results, they constructed ad hoc sub-grid models for the effects of the fine-scale flow structures on the drag force and the stresses, and examined the consequence of these sub-grid models on the outcome of the coarsegrid simulations of gas-particle flow in a large-scale vertical riser. They demonstrated that these sub-grid scale corrections affect the predicted large-scale flow patterns profoundly. ${ }^{31}$

Thus, it is clear that one must carefully examine whether a microscopic two-fluid model must be modified to introduce the effects of unresolved structures before embarking on coarsegrid simulations of gas-particle flows. In the study by Andrews et al. ${ }^{31}$, the filtering was done simply by choosing the filter size to be the grid size in the coarse-grid simulation of the filtered equations. Furthermore, the corrections accounting for the effects of the structures which would 
not be resolved in the coarse-grid simulations were extracted from highly resolved simulations performed in a periodic domain whose size was chosen to be the same as the filter size; this imposed periodicity necessarily limited the dynamics of the structures in the highly resolved structures and so the accuracy of using the sub-grid models deduced from such restrictive simulations is debatable.

The first objective of the present study is to develop a systematic filtering approach and construct closure relationships for the drag coefficient and the effective stresses in the gas and particle phases that are appropriate for coarse-grid simulations gas-particle flows. Briefly, we have performed highly resolved simulations of a kinetic theory based two-fluid model in a large periodic domain, and analyzed the results using different filter sizes. In this case, as the filter size is considerably smaller than the periodic domain size, the microstructures sampled in the filtered region are not constrained by the periodic boundary conditions. The present approach also exposes nicely the filter size dependence of various quantities.

It should be emphasized that the present study does not challenge the validity of the microscopic two-fluid model equations such as the kinetic theory based equations. Instead, it uses these microscopic equations as a starting point and seeks modifications to make them suitable for coarse-grid simulations. (If a fine grid can be used to resolve all the structures contained in the microscopic two-fluid model equations, the present analysis is unnecessary; however, such high resolution is neither practical nor desirable for the analysis of the macroscale flow behavior.)

The second objective of the present study is to demonstrate that filtering does indeed remove small scale structures that are afforded by the microscopic two-fluid models. If filtering 
has been done in a meaningful manner, the filtered equations should yield coarser structures than the microscopic two-fluid model (from which the filtered equations were derived) even when one uses fine spatial resolution. We will demonstrate that this is indeed the case.

In the body of the paper, the microscopic two-fluid model equations employed in our simulations are briefly discussed in $\$ 2$. The coarse-grained two-fluid model equations are presented in $\S 3$. The closure relations for the filtered model equations, obtained by filtering the computational data generated via highly resolved simulations of the microscopic two-fluid model equations, are examined in $\S 4$. The 1D liner stability analyses of the filtered two-fluid models are given in $\S 5$ and the findings of this study are summarized in $\S 6$.

\section{Microscopic Two-fluid Model Equations}

The general form of the two-fluid model equations for gas-particle flows is fairly standard. However, several choices have been discussed and analyzed in the literature for the constitutive relations for the fluid-particle drag force and the effective stresses. ${ }^{2,3,12}$ We consider a system consisting of uniformly sized particles and focus on the situation where the particles interact only through binary collisions. In the kinetic theory approach, the continuity and momentum equations for the gas and particle phases are supplemented by an equation describing the evolution of the fluctuation energy (a.k.a. granular energy) associated with the particles, which is used to compute the local granular temperature; the particle phase stress is then expressed in terms of the local particle volume fraction, granular temperature, rate of deformation and particle properties. There are several different closures for the terms appearing in the granular energy equation as well. Thus, it must be emphasized that while the general forms of the continuity, momentum and granular energy equations are common among most of the 
microscopic two-fluid models discussed in the literature, there are variations in the closure relations. Thus, the exact form of the closures for the microscopic two-fluid model is still evolving. Nevertheless, the microscopic two-fluid models are robust in the sense that when they are augmented with physically reasonable closures, they do yield all the known instabilities in gas-particle flows, which in turn lead to persistent fluctuations that take the form of bubble-like voids in dense fluidized beds and clusters and streamers in dilute systems. ${ }^{2,3,30,32,40-46}$ Thus, all sets of constitutive relations which capture these small scale instabilities can be expected to lead to similar conclusions regarding the structure of the closures for the filtered equations. With this in mind, we have selected one set of closures for the kinetic theory based microscopic equations (see Table 1). Further discussion of these equations and an extensive review of the relevant literature can be found in Agrawal et al. ${ }^{30}$. As the closures for the microscopic two-fluid models improve, one can easily repeat the analysis described here and refine the filtered closures.

\section{Filtered Two-fluid Model Equations}

We coarse-grain the two-fluid model equations through a filtering operation which amounts to spatial averaging over some chosen filter length scale. In these filtered (a.k.a. coarsegrained) equations, the consequences of the flow structures occurring on a scale smaller than a chosen filter size appear through residual correlations for which one must derive or postulate constitutive models. If constructed properly, and if the several assumptions innate to the filtering methodology hold true, the filtered equations should produce a solution with the same macroscopic features as the finely resolved kinetic theory model solution; however obtaining this solution should come at less computational cost. 
Let $\phi_{s}(\mathbf{y}, t)$ denote the particle volume fraction at location $\mathbf{y}$ and time $t$ obtained by solving the microscopic two-fluid model. We can define a filtered particle volume fraction $\overline{\phi_{s}}(\mathbf{x}, t)$ as

$$
\bar{\phi}_{s}(\mathbf{x}, t)=\int_{V_{\infty}} G(\mathbf{x}, \mathbf{y}) \phi_{s}(\mathbf{y}, t) \mathrm{d} \mathbf{y}
$$

where $G(\mathbf{x}, \mathbf{y})$ is a weight function that depends on $\mathbf{x}-\mathbf{y}$ and $v_{\infty}$ denotes the region over which the gas-particle flow occurs. The weight function satisfies $\int_{V_{\infty}} G(\mathbf{x}, \mathbf{y}) \mathrm{d} \mathbf{y}=1$. By choosing how rapidly $G(\mathbf{x}, \mathbf{y})$ decays with distance measured from $\mathbf{x}$, one can change the filter size. We define the fluctuation in particle volume fraction as

$$
\phi_{s}^{\prime}(\mathbf{y}, t)=\phi_{s}(\mathbf{y}, t)-\overline{\phi_{s}}(\mathbf{y}, t)
$$

Filtered phase velocities are defined according to

$$
\overline{\phi_{s}}(\mathbf{x}, t) \overline{\mathbf{v}}(\mathbf{x}, t)=\int_{V_{\infty}} G(\mathbf{x}, \mathbf{y}) \phi_{s}(\mathbf{y}, t) \mathbf{v}(\mathbf{y}, t) \mathrm{d} \mathbf{y}
$$

and

$$
\left(1-\overline{\phi_{s}}(\mathbf{x}, t)\right) \overline{\mathbf{u}}(\mathbf{x}, t)=\int_{V_{\infty}} G(\mathbf{x}, \mathbf{y})\left(1-\phi_{s}(\mathbf{y}, t)\right) \mathbf{u}(\mathbf{y}, t) \mathrm{d} \mathbf{y}
$$

Here, $\mathbf{u}$ and $\mathbf{v}$ denote local gas and particle phase velocities appearing in the microscopic twofluid model. We then define the fluctuating velocities as:

$$
\mathbf{v}^{\prime}(\mathbf{y}, t)=\mathbf{v}(\mathbf{y}, t)-\overline{\mathbf{v}}(\mathbf{y}, t) \quad \text { and } \quad \mathbf{u}^{\prime}(\mathbf{y}, t)=\mathbf{u}(\mathbf{y}, t)-\overline{\mathbf{u}}(\mathbf{y}, t)
$$


Applying such a filter to the continuity equations (1 and 2) in Table 1, we obtain

$$
\frac{\partial \rho_{\mathrm{s}} \overline{\phi_{s}}}{\partial \mathrm{t}}+\nabla \cdot\left(\rho_{\mathrm{s}} \overline{\phi_{s}} \overline{\mathbf{v}}\right)=0
$$

and

$$
\frac{\partial\left(\rho_{\mathrm{g}}\left(1-\overline{\phi_{s}}\right)\right)}{\partial \mathrm{t}}+\nabla \cdot\left[\rho_{\mathrm{g}}\left(1-\overline{\phi_{s}}\right) \overline{\mathbf{u}}\right]=0
$$

as the filtered continuity equations, where we have assumed that the gas density does not vary appreciably over the representative region of the filter. These are identical in form to the microscopic continuity equations in Table 1 . Repeating this analysis with the two microscopic momentum balance equations ( 3 and 4 in Table 1), we obtain the following filtered momentum balances:

$$
\begin{gathered}
{\left[\frac{\partial\left(\rho_{\mathrm{s}} \overline{\phi_{s}} \overline{\mathbf{v}}\right)}{\partial \mathrm{t}}+\nabla \cdot\left(\rho_{\mathrm{s}} \overline{\phi_{s}} \mathbf{\mathbf { v }}\right)\right]=\quad-\nabla \cdot \sum_{s}-\bar{\phi} \nabla \cdot \overline{\boldsymbol{\sigma}}_{g}+\overline{\mathbf{F}}+\rho_{\mathrm{s}} \overline{\phi_{s}} \mathbf{g}} \\
{\left[\frac{\partial\left(\rho_{g}\left(1-\overline{\phi_{s}}\right) \overline{\mathbf{u}}\right)}{\partial \mathrm{t}}+\nabla \cdot\left(\rho_{g}\left(1-\overline{\phi_{s}}\right) \overline{\mathbf{u} \mathbf{u}}\right)\right]=-\left(1-\overline{\phi_{s}}\right) \nabla \cdot \overline{\boldsymbol{\sigma}}_{g}-\nabla \cdot\left(\rho_{g} \overline{\left(1-\phi_{s}\right) \mathbf{u}^{\prime} \mathbf{u}^{\prime}}\right)} \\
-\overline{\mathbf{F}}+\rho_{g}\left(1-\overline{\phi_{s}}\right) \mathbf{g}
\end{gathered}
$$

Here

$$
\begin{aligned}
& \sum_{s}=\overline{\boldsymbol{\sigma}}_{s}+\rho_{s} \overline{\phi_{s} \mathbf{v v}}-\rho_{s} \overline{\phi_{s}} \overline{\mathbf{v}} \overline{\mathbf{v}}=\overline{\boldsymbol{\sigma}}_{s}+\rho_{s} \overline{\phi_{s} \mathbf{v}^{\prime} \mathbf{v}^{\prime}} \\
& \overline{\mathbf{F}}=\overline{\mathbf{f}}-\overline{\phi_{s}^{\prime} \nabla \cdot \boldsymbol{\sigma}_{g}^{\prime}}
\end{aligned}
$$


The filtered momentum balance equations are nearly identical (in form) to the microscopic momentum balances in Table 1. One exception is that the filtered gas phase momentum balance now contains an additional term, $\nabla \cdot\left(\rho_{g} \overline{\left(1-\phi_{s}\right) \mathbf{u}^{\prime} \mathbf{u}^{\prime}}\right)$. In the class of problems we consider here the contribution of this term is much weaker than the first and third terms on the right hand side of the gas phase momentum balance equation (as $\rho_{s} \phi_{s}>>\rho_{g}\left(1-\phi_{s}\right)$ in most of the flow domain in the problem considered here).

The effective particle phase stress, $\Sigma_{s}$, includes the filtered microscopic stress $\overline{\boldsymbol{\sigma}}_{s}$ and a Reynolds stress-like contribution coming from the particle phase velocity fluctuations, see equation (17). As noted by Agrawal et al. ${ }^{30}$ and Andrews et al. ${ }^{31}$, the contribution due to the velocity fluctuations dwarf the microscopic particle phase stress even for modestly large filter sizes and this will be seen clearly in the results presented below. Thus, when realistically large filter sizes (of the order of 100 particle diameters or more) are employed, one can neglect the $\overline{\boldsymbol{\sigma}}_{s}$ contribution for all practical purposes for the particle volume fraction range analyzed in this study. Therefore, at least as a first approximation, it is not necessary to include a filtered granular energy equation in the analysis. ${ }^{31}$ This statement, however, does not imply that the granular energy equation (see (5) in Table 1) is not important in gas-particle flows. The granular energy equation and the parameters (such as the coefficient of restitution) contained in it can certainly be expected to influence the details of the small scale structures, which in turn affect the velocity fluctuation term in the filtered particle phase stress.

The filtered gas-particle interaction force $\overline{\mathbf{F}}$ includes a filtered gas-particle drag force $\overline{\mathbf{f}}$ and a term representing correlated fluctuations in particle volume fraction and the (microscopic two-fluid model) gas phase stress gradient, see equation (18). 
Before one can analyze the filtered two-fluid model equations, constitutive relations are needed for the residual correlations $\overline{\mathbf{F}}, \Sigma_{s}$ and $\overline{\boldsymbol{\sigma}}_{g}$ in terms of filtered particle volume fraction, velocities and pressure. Furthermore, as these are filtered quantities, the constitutive relations capturing them will necessarily depend on the details of the fluctuations being averaged, but these details will depend on the location in the process vessel. For example, one can anticipate that fluctuations in the vicinity of solid boundaries will be different from those away from such boundaries. Accordingly, it is entirely reasonable to expect that the constitutive models for these residual correlations should include some dependence on distance from boundaries. (This is well known in single phase turbulent flows.) In the present study, we do not address the boundary effect, but focus on constitutive models that are applicable in regions away from boundaries as it is an easier first problem to address. It is assumed that the constitutive relations for the residual correlations will depend on local filtered variables and their gradients.

In rapid gas-particle flows with $\rho_{s} \phi_{s} \gg \rho_{g}\left(1-\phi_{s}\right)$, it is invariably the case that $\rho_{g} \overline{\left(1-\phi_{s}\right) \mathbf{u}^{\prime} \mathbf{u}^{\prime}}<<\rho_{s} \overline{\phi_{s} \mathbf{v}^{\prime} \mathbf{v}^{\prime}}$, and we simplify the filtered gas phase stress as:

$$
\overline{\boldsymbol{\sigma}}_{g} \approx-\overline{\mathrm{p}_{\mathrm{g}}} \mathbf{I}
$$

We express $\overline{\mathbf{F}}$ as

$$
\overline{\mathbf{F}}=\overline{\mathbf{f}}-\overline{\phi_{s}^{\prime} \nabla \cdot \boldsymbol{\sigma}_{g}^{\prime}}=\overline{\beta_{e}}(\overline{\mathbf{u}}-\overline{\mathbf{v}})
$$

where $\overline{\beta_{e}}$ is a filtered drag coefficient to be found. The $\overline{\overline{\phi_{s}^{\prime} \nabla \cdot \boldsymbol{\sigma}_{g}^{\prime}}}$ term in equation (20) can also add a dynamic part, resembling an apparent added mass force ${ }^{40-42}$; however, as Andrews ${ }^{43}$ found 
such a dynamic part to be of much smaller than the drag force term in equation (20), we will limit ourselves to equation (20).

We begin our analysis by postulating the following filtered particle phase stress model:

$$
\Sigma_{s}=\overline{p_{s e}} \mathbf{I}-\overline{\mu_{b e}}(\nabla \cdot \overline{\mathbf{v}}) \mathbf{I}-\overline{\mu_{s e}}\left(\nabla \overline{\mathbf{v}}+(\nabla \overline{\mathbf{v}})^{\mathrm{T}}-\frac{2}{3}(\nabla \cdot \overline{\mathbf{v}}) \mathbf{I}\right)
$$

where $\overline{p_{s e}}\left(=\overline{p_{s}}+\frac{1}{3}\left(\rho_{s} \overline{\phi_{s} \mathrm{v}_{\mathrm{x}}^{\prime} \mathrm{v}_{\mathrm{x}}^{\prime}}+\rho_{s} \overline{\phi_{s} \mathrm{v}_{\mathrm{y}}^{\prime} \mathrm{v}_{\mathrm{y}}^{\prime}}+\rho_{s} \overline{\phi_{s} \mathrm{v}_{\mathrm{z}}^{\prime} \mathrm{v}_{\mathrm{z}}^{\prime}}\right)\right)$ is the filtered particle phase pressure; $\overline{\mu_{s e}}$ and $\overline{\mu_{b e}}$ are the filtered particle phase shear and bulk viscosity, respectively. As our simulations described below do not permit an evaluation of $\overline{\mu_{b e}}$, we do not consider this term in the present analysis and we simplify equation (21) as

$$
\sum_{s} \approx \overline{p_{s e}} \mathbf{I}-\overline{\mu_{s e}}\left(\nabla \overline{\mathbf{v}}+(\nabla \overline{\mathbf{v}})^{\mathrm{T}}-\frac{2}{3}(\nabla \cdot \overline{\mathbf{v}}) \mathbf{I}\right)
$$

The filtered particle phase shear viscosity is defined as $\overline{\mu_{s e}}=\rho_{s} \frac{\overline{\phi_{s} \mathrm{v}_{x}^{\prime} \mathrm{v}_{y}^{\prime}}}{\overline{\frac{\partial \mathrm{v}_{x}}{\partial y}}+\frac{\overline{\partial \mathrm{v}_{y}}}{\partial x}}+\overline{\mu_{s}}$.

We now seek closure relations for $\overline{\beta_{e}}, \overline{p_{s e}}$ and $\overline{\mu_{s e}}$ by filtering computational data gathered from highly resolved simulations of the microscopic two-fluid model equations.

\section{Detailed solution of microscopic two-fluid model equations}

As already noted in $\S 1$, we restrict our attention to closures for $\overline{\beta_{e}}, \overline{p_{s e}}$ and $\overline{\mu_{s e}}$ in flow regions far away from solid boundaries. A simple and effective manner by which solid boundaries can be avoided is to consider flows in periodic domains. The filtering operation does 
not require a periodic domain; however, as each location in a periodic domain is statistically equivalent to any other location, statistical averages can be gathered much faster when simulations are done in periodic domains. With this in mind, all the analyses described here have been performed in periodic domains. Agrawal et al. ${ }^{30}$ have already shown that the results obtained from 2-D and 3-D periodic domains are qualitatively similar, but differ somewhat quantitatively; therefore, we have focused first on 2-D simulations in the present study to bring forward the filter size dependence of the closures for the residual correlations, as 2-D simulations are computationally less expensive. We will present several 3-D simulation results at the end to bring forth the differences between 2-D and 3-D closures.

\subsection{Two-dimensional simulations}

We have performed many sets of highly resolved simulations (of the set of microscopic two-fluid model equations for a fluidized suspension of particles presented in Table 1) in large 2D periodic domains. All of our simulations were performed using the open-source software MFIX ${ }^{5}$. These simulations are identical to those described by Agrawal et al. ${ }^{30}$, except that our simulations are now done for much larger domain sizes. Agrawal et al..$^{30}$ averaged the residual correlations over the entire domain (i.e., the filter size is the same as their domain size), but as our simulation domains are much larger, the computationally generated "data" can now be averaged using a range of filter sizes that are smaller than the domain size.

After an initial transient period which depends on the initial conditions, persistent, timedependent and spatially inhomogeneous structures develop. Figure 2 shows an instantaneous snapshot of the particle volume fraction field in one such 2-D simulation and the cells (i.e., fine grids) used in the simulations. One can then select any region of desired size (illustrated in the 
figure as gray squares of different sizes) and average any quantity of interest over all the cells inside that region; we refer to such results as region-average (or filtered) values. (Such region averaging is equivalent to setting the weight function to an appropriate non-zero constant everywhere inside the region and to zero outside.) Note that one can choose a large number of different regions of the same size inside the overall domain and thus many region-averaged values can be extracted from each instantaneous snapshot. When the system is in a statistical steady state, one can construct tens of thousands of such averages by repeating the analysis at various time instants.

Returning to figure 2, note that the averages over different regions at any given time are not equivalent; for example, at the given instant, different regions (even of the same size) will correspond to different region-averaged particle volume fractions, particle and fluid velocities. Thus, one cannot simply lump the results obtained over all the regions; instead, we must group them into bins based on various markers and perform statistical averages within each bin to extract useful information. Our 2-D simulations revealed that the single most important marker for a region is its average particle volume fraction. Therefore, we divided the permissible range of filtered particle volume fraction $\left(0 \leq \bar{\phi}_{s}<\phi_{s, \max }=0.65\right)$ into 1300 bins (so that each bin represented a volume fraction window of 0.0005 ) and classified the filtered data in these bins. (Strictly speaking, one would expect to use two-dimensional bins, involving $\overline{\phi_{s}}$ and a Reynolds number based on slip velocity, to classify the filtered drag coefficient; however, we found the Reynolds number dependence to be rather weak for the cases investigated in this study.) For each snapshot of the flow field in the statistical steady state, we considered a filtering region around each grid point in the domain and determined the filtered particle volume fraction $\bar{\phi}_{s}$, filtered 
slip velocity $(\overline{\mathbf{u}}-\overline{\mathbf{v}})$, filtered fluid-particle interaction force, etc. This combination of filtered quantities represents one realization and it was placed in the appropriate filtered particle volume fraction bin, determined by its volume fraction value. In this manner a large number of realizations could be generated from each snapshot. This procedure was repeated for many snapshots. The many realizations within each bin were then averaged to determine ensembleaveraged values for each filtered quantity. From such bin statistics, we calculated the filtered drag coefficient, the filtered particle phase normal stresses and filtered particle phase viscosity as functions of filtered particle volume fraction. For example, the filtered drag coefficient is taken to be the ratio of the filtered drag force and the filtered slip velocity, each of which has been determined in terms of the volume fraction. All the results are presented as dimensionless variables, with $\rho_{\mathrm{s}}, \mathrm{v}_{\mathrm{t}}$ and $g$ representing characteristic density, velocity and acceleration.

Figure 3 shows the variation of the dimensionless filtered drag coefficient, $\overline{\beta_{e, d}}=\left(\overline{\beta_{e}} \mathrm{v}_{\mathrm{t}} / \rho_{s} g\right)$ as a function of $\overline{\phi_{s}}$ for one particular filter size. Even though all the results are presented in terms of dimensionless units, it is instructive to consider some dimensional quantities to help visualize the physical problem better. Most of the 2-D filtered results presented in this manuscript are based on computational data gathered in a $131.584 \times 131.584$ (dimensionless units) square periodic domain; this domain size translates to $0.64 \mathrm{~m} \times 0.64 \mathrm{~m}$ for the FCC particles (whose physical properties are given in Table 2). The dimensionless filter size of 8.224 used in figure 3 corresponds to a filter size of $0.04 \mathrm{~m}$ for the FCC particles. Thus, one can readily appreciate that this filter size is quite small compared to the macroscopic dimensions of typical process vessels. The various symbols in this figure refer to computational data obtained by solving the microscopic two-fluid model equations at different resolution levels. 
Simulations were performed using different domain-average particle volume fractions so that every (filtered) volume fraction shown here would have many realizations. This figure indicates that at a sufficiently high resolution the results did become nearly independent of the grid size used in the simulations to generate the computational data. Typically, when the grid size was smaller than the filter size by a factor of four or more (so that there were at least 16 grids inside the filtering region in 2-D simulations), the filtered results were found to be essentially independent of the grid resolution.

The effect of (periodic) domain size on the filtered drag coefficient was explored by performing simulations with two different domain sizes. Figure 4 presents the dimensionless filtered drag coefficient for two different filter sizes and two different domain sizes. It is clear that for both filter sizes the results are essentially independent of domain size. In general, we found that the filtered results were independent of the domain size as long as the filter size was smaller than $1 / 4^{\text {th }}$ of the domain size. (The filter size dependence seen in this figure is discussed below.)

The results presented in figures 3 and 4 , and in many of the figures below, were generated by combining results obtained from simulations with many different specified domainaverage particle volume fractions. Figure 5 shows the variation of the filtered drag coefficient with filtered particle volume fraction, with results obtained from simulations with different domain-average particle volume fractions indicated with different symbols. Although the domain-average particle volume fraction affects the filtered drag coefficient slightly (particularly for volume fractions above $\sim 0.20$ ), this effect is clearly much smaller than that of the filter size. Physically, this implies that the filter size dependence manifested in this and other figures largely stems from the inhomogeneous microstructure inside the filtering region and the filtered drag 
coefficients are either independent of or only weakly dependent on the conditions prevailing outside the filtered region (at least over the range of particle volume fractions over which the data were collected).

Figure 6a shows the variation of the (dimensionless) filtered drag coefficient, as a function of filtered particle volume fraction for various filter sizes. The results for the two smallest filter sizes are likely to decrease somewhat if simulations could be done at higher resolutions, but as noted earlier in the context of figure 3 the results for all larger filter sizes are essentially independent of grid size. It is clear that the filtered drag coefficient decreases substantially with increasing filter size, and this can readily be rationalized. As we increase the filter size, averaging is being performed over larger and larger clusters - larger clusters allow greater bypassing of the gas resulting in lower apparent drag coefficient. The uppermost curve in figure 6a is the intrinsic drag law; the filter size here is simply the grid size used in the simulations of the microscopic two-fluid model equations (which is equivalent to no filtering at all). For typical FCC particles (see Table 2), a dimensionless filter size of 2.056 is equivalent to $0.01 \mathrm{~m}$, and so even at small filter sizes (from an engineering viewpoint) an appreciable reduction occurs in the effective drag coefficient.

An alternative presentation of the filtered drag coefficient is given in Figure 6b. It is clear that in figure $6 a$ the variation of the filtered drag coefficient with particle volume fraction is dictated by that of the microscopic drag coefficient. Figure $6 \mathrm{~b}$ shows how the ratio of the filtered drag coefficient to the microscopic drag coefficient changes with particle volume fraction for several filter lengths. Our major observation from the figure is that this ratio is weakly dependent on the particle volume fraction for the range of $0.03<\phi_{s}<0.30$. The ratio within this range can 
be represented as a function of the filter length only. It can be given in a simple algebraic form as

$$
\overline{\beta_{e}}=\frac{32 F r_{f}^{-2}+63.02 F r_{f}^{-1}+129}{F r_{f}^{-3}+133.6 F r_{f}^{-2}+66.61 F r_{f}^{-1}+129} \beta
$$

Intuitively, one can expect that the clusters will not grow beyond some critical size and that at sufficiently large filter sizes the filtered drag coefficient will become essentially independent of the filter size. It is clear from Figure $6 \mathrm{a}$ and $6 \mathrm{~b}$ that this critical filter size is definitely larger than the largest filter size shown there. Simulations using much larger domains are needed to identify this critical size, but we have not pursued this issue in the present study; instead, we have focused on a qualitative understanding as to how the filtered quantities depend on filter size for modest filter sizes.

It was seen earlier, Eq. (18), that the filtered drag force includes contributions from two terms. The second term is essentially equal to $-\overline{\phi^{\prime} \nabla p_{g}^{\prime}}$ as the deviatoric stress in the gas phase is quite small. The contribution from this term to the filtered drag coefficient is presented in Figure 7, while the total contribution due to both terms was shown earlier in Figure 6a. While the overall filtered drag coefficient decreases with increasing filter size (Figure 6a), the contribution from $-\overline{\phi^{\prime} \nabla p_{g}^{\prime}}$ first increases with the filter size and then decreases (Figure 7). However, $-\overline{\phi^{\prime} \nabla p_{g}^{\prime}}$ contributes no more than $25 \%$ of the overall filtered drag coefficient. So, over this range of filter sizes, the primary contribution to $\overline{\mathbf{F}}$ comes from $\overline{\mathbf{f}}$.

The results presented in Figure 6a are plotted in Figure 8 on a logarithmic scale which shows: (a) the typical Richardson-Zaki ${ }^{44}$ form for $\phi_{s}$ not too close to zero, and (b) at small $\bar{\phi}_{s}$ 
values, a clear departure from this trend. The uppermost curve in this figure corresponds to the intrinsic drag expression extracted simply using a filter size equal to the grid size of the simulations. The two obvious regions manifested by this uppermost curve can be traced to a Reynolds number $\left(\operatorname{Re}_{g}\right)$ effect present in the Wen and $\mathrm{Yu}^{6}$ drag expression used in the simulations. The filtered slip velocity in the vertical direction, as a function of $\overline{\phi_{s}}$, is shown in Figure 9 for various filter sizes. Here the bottommost curve is for the case where the filter size is the same as the grid size; the inverse relationship between the local slip velocity and the particle volume fraction is clear. It can be seen from Equation (7) that increases with $\phi_{s}$ and $\mathrm{Re}_{g}$; in the uppermost curve in figure 8, the effect of $\phi_{s}$ dominates at high $\phi_{s}$ values while the $\operatorname{Re}_{g}$ effect leads to a reversal of trend at very small $\phi_{s}$ values. To establish this point, we carried out simulations where the intrinsic drag coefficient expression (see Equation 7) was modified by setting $C_{D}=24 / \operatorname{Re}_{g}$ (so that only the Stokes drag remained). Figure 10 shows the results obtained from these simulations, $c f$. Figure 8. The uppermost curve in Figure 10 does not show the reversal of trend at very small $\phi_{s}$ values, establishing Reynolds number effect as the reason for the difference between the shapes of the uppermost curves in Figures 8 and 10.

Let us now consider the other curves in both Figures 8 and 10, which are for filter sizes larger than grid size. All of these curves exhibit a Richardson-Zaki like behavior at high volume fractions and a reversal of trend at very low particle volume fractions. This behavior is not due to an $\operatorname{Re}_{g}$ effect in the intrinsic drag law, as Figure 10 does not have any such dependence, and so one has to seek an alternate explanation. The results presented in Figure 9 indicate that one cannot capture this effect through a Reynolds number term involving the filtered slip velocity. 
Note that for large filter sizes, the slip velocity manifests a peak at some intermediate $\bar{\phi}_{s}$; for $\bar{\phi}_{s}$ values to the left of this peak, the filtered slip velocity decreases as $\bar{\phi}_{s}$ is decreased, while the quantity plotted in figures 8 and 10 increase with decreasing $\bar{\phi}_{s}$. Thus, if we seek to capture the data in Figures 8 and 10 in the low $\overline{\phi_{s}}$ region through a Reynolds number dependence (based on the filtered slip velocity), it will involve a negative order dependence, which makes no physical sense. Therefore we attribute the trend reversal seen in Figures 8 and 10 at small $\bar{\phi}_{s}$ values to just the inhomogeneous microstructure inside the filter region. At low $\overline{\phi_{s}}$ values, an increase in $\overline{\phi_{s}}$ increases both the cluster size and particle volume fraction in the clusters; the gas flows around these clusters and the resistance offered by these clusters decreases with increasing cluster size. Large filter sizes average over larger clusters and so the extent of drag reduction observed increases with filter size. At sufficiently large $\overline{\phi_{s}}$ values, the clusters begin to interact and hindered drag sets in. This behavior is clearly reflected in the vertical slip velocity corresponding to large filter sizes, see Figure 9 . The slip velocity increases with $\overline{\phi_{s}}$ at small $\overline{\phi_{s}}$ values, consistent with larger and/or denser clusters; it then decreases with increasing $\bar{\phi}_{s}$ when the clusters begin interact with each other.

It is interesting to note in Figure 9 that the dimensionless slip velocity, in the limit of zero particle volume fraction ( $\overline{\phi_{s}} \rightarrow 0$ ), differs from unity. In our simulations with various domainaverage particle volume fractions, regions with $\overline{\phi_{s}} \rightarrow 0$ appeared in the dilute phase surrounding the clusters; here the slip velocity was almost always larger than the terminal velocity. This implies that the gas in the dilute phase was constantly engaged in accelerating the particles 
upward. This can happen only if the clusters are dynamic in nature with active, continual exchange of particles between the clusters and the dilute phase.

Linear fits of the data in Figure 8 over the particle volume fraction range ( $0.10 \leq \bar{\phi}_{s} \leq 0.30$ ) were used to estimate dimensionless apparent terminal velocity $V_{t, a p p}$ and an apparent Richardson-Zaki exponent, $N_{R Z, a p p}$.

$$
\ln \left(\frac{\overline{\beta_{e}} \mathrm{v}_{\mathrm{t}}}{\rho_{s} g \overline{\phi_{s}}\left(1-\bar{\phi}_{s}\right)}\right)=\ln \left(\frac{\overline{\beta_{e, d}}}{\overline{\phi_{s}}\left(1-\overline{\phi_{s}}\right)}\right)=-\left(N_{R Z, a p p}-1\right) \ln \left(1-\overline{\phi_{s}}\right)-\ln \left(V_{t, a p p}\right) .
$$

The variation of $V_{t, a p p}$ and $N_{R Z, a p p}$ with dimensionless filter size, $F r_{f}^{-1}=g \Delta_{f} / \mathrm{v}_{\mathrm{t}}^{2}$, are shown in Figures 11a (diamonds) and 11b, respectively. Here $\Delta_{f}$ denotes the filter size. Both increase with filter size.

Figure 12a shows the variation with $\bar{\phi}_{s}$ of the dimensionless filtered kinetic theory pressure, $\overline{p_{s, d}}=\overline{p_{s}} / \rho_{s} \mathrm{v}_{\mathrm{t}}^{2}$, for the simulations discussed earlier in connection with Figures 4 and 8. At very low $\bar{\phi}_{s}$ values the filtered kinetic theory pressure is essentially independent of filter size, but at larger $\bar{\phi}_{s}$ values distinct filter size dependence becomes clear. Figure $12 \mathrm{~b}$ shows the dimensionless total particle phase pressure $\overline{p_{s e, d}}=\overline{p_{s e}} / \rho_{s} \mathrm{v}_{\mathrm{t}}^{2}$ as a function of $\overline{\phi_{s}}$ for various filter sizes. Here the filtered particle phase pressure includes the pressure arising from the streaming and collisional parts captured by the kinetic theory and the sub-filter-scale Reynolds-stress like velocity fluctuations (see text below Equation 21). Comparing Figures 12a and 12b, we see that the contributions resulting from the sub-filter-scale velocity fluctuations swamp the kinetic theory pressure indicating that, at the coarse-grid scale, one can ignore the kinetic theory 
contributions to the pressure. It is also clear from Figure 12b that the filtered pressure increases with filter size, a direct consequence of the fact that the energy associated with the velocity fluctuations increases with filter length (as in single phase turbulence). Once again, results obtained from simulations with different domain-average particle volume fractions collapse on to the same curves (as earlier in Figures 5 and 6 for the filtered drag coefficient), confirming that the filtered quantities largely depend on quantities inside the filtering region. The data presented in Figure $12 \mathrm{~b}$ could be captured by an expression of the form $a \phi_{s}\left(1-b \phi_{s}\right)$ with $b \sim 1.80$. The parameter $a$ increases with filter size, see Figure 12c.

Figures $13 \mathrm{a}$ and $13 \mathrm{~b}$ show the variation with $\bar{\phi}_{s}$ of dimensionless filtered kinetic theory viscosity, $\overline{\mu_{s, d}}=\overline{\mu_{s}} g / \rho_{s} \mathrm{v}_{\mathrm{t}}^{3}$, and the (dimensionless) filtered particle phase shear viscosity, $\overline{\mu_{s e, d}}=\overline{\mu_{s e}} g / \rho_{s} \mathrm{v}_{\mathrm{t}}^{3}$. The latter includes the streaming and collisional parts captured by the kinetic theory (shown in Figure 13a) and that associated with the sub-filter-scale velocity fluctuations. It is readily seen that for large filter sizes, the contribution from the sub-filter scale velocity fluctuations dominate, and the filtered particle phase viscosity increases appreciably with filter size. Once again, results from simulations with different domain-average particle volume fractions collapse on the same curves, adding further support to the viability of the filtering approach. The data presented in Figure 13b could be captured by an expression of the form $c \phi_{s}\left(1-d \phi_{s}\right)$ with $d \sim 0.86$. The parameter $c$ increases with filter size, see Figure 13c.

It is mentioned in passing that we have studied the robustness of the filtered statistics against small changes in the secondary model parameters (namely, the coefficient of restitution, density ratio, etc.) and found that they are much less important than the dimensionless filter size; 
so, capturing the effect of the dimensionless filter size on the dimensionless filtered drag coefficient is indeed the most important challenge.

Agrawal et $a .^{30}$ and Andrews et al. ${ }^{31}$ determined domain-averaged drag coefficient, particle phase pressure and viscosity by averaging their kinetic theory simulation results over the entire periodic domain. In contrast, we have performed the averaging over regions that are much smaller than the periodic domain, so that the filtered statistics are not affected by the periodic boundary conditions. It is interesting to observe that the filter size dependences of all these filtered quantities obtained in our study are qualitatively identical to those reported in the studies of Agrawal et al. ${ }^{30}$ and Andrews et al. ${ }^{30}$ This further confirms that the robustness of the role played by filter size.

\subsection{Three-dimensional simulations}

Figure 14 shows a snapshot of the particle volume fraction field in a 3-D periodic domain, and the presence of particle-rich strands is readily visible. Figure 15 shows the effect of grid resolution on the filtered drag coefficient. As seen earlier in Figure 3 for 2-D simulations, the dependence of the filtered drag coefficient on grid resolution becomes weaker as the filter size increases. At the lower grid resolution, the filter size of 1.028 is the same as the grid size and when the grid resolution is increased, the filtered drag coefficient changes appreciably. For a filter size of 4.112, there are 512 and 4096 grids inside the filter volume in the two simulations; these are quite large and so the filtered drag coefficient manifests only a weak dependence on resolution. 
Figure 16 displays the variation of filtered drag coefficient with particle volume fraction for different filter sizes. As the grid size used in these simulations is 0.257 dimensionless units, the uppermost curve corresponds to using no filter at all. The next curve corresponding to the filter size of 0.514 has only 8 grids inside the filtering volume and so is likely to change if simulations with greater resolutions are performed. The results for other, larger filter sizes are expected to be nearly independent of grid resolution. It is clear from Figure 16 that the filter size dependence of the filter drag coefficient seen earlier in the 2-D simulations persist in 3-D as well.

As in the case of 2-D simulations, the filtered drag coefficient obtained from 3-D simulations at different domain-average particle volume fractions collapse onto the same curve (over the range of volume fractions displayed), see Figure 17. Furthermore, Figure 18 illustrates the filtered drag coefficient is indeed independent of the domain size. These suggest that the filtered drag coefficient is largely determined by the inhomogeneous microstructure inside the filtering volume. The results presented in Figure 16 are plotted on a natural logarithmic scale in Figure 19. Richardson-Zaki like behavior at high particle volume fractions and a reversal of the trend at lower volume fractions, seen earlier in 2-D simulations (see Figure 8), persist in 3-D as well. Filter size dependence of the apparent terminal velocity and the exponent in the Richardson-Zaki regime, are shown in Figures 11a and 11c. The apparent terminal velocity increases with filter size, just as it did for 2-D simulations; however, the Richardson-Zaki exponent shows a slight decline with increasing filter size, in marked contrast to 2-D simulations (see Figure 11b). Thus, there are definite quantitative differences between 2-D and 3-D results; however, it is clear from Figures 8 and 19 that both 2-D and 3-D results are strikingly similar. 
Figures 20 and 21 present filtered particle phase pressure and viscosity extracted from 3D simulations and can be compared to Figures $12 \mathrm{~b}$ and $13 \mathrm{~b}$, respectively. The strong filter size dependence of these quantities is clearly present in both two- and three- dimensions.

\section{Sample solution of the filtered two-fluid model equations}

Although a fundamentally based, quantitative model for the dependence of $\overline{\beta_{e}}, \overline{p_{s e}}$ and $\overline{\mu_{s e}}$ on filter size and $\overline{\phi_{s}}$ presented above remains elusive at the present time, one can interrogate whether the filtered two-fluid model approach is scientifically sound. Ultimately, models should be validated against experimental data; however, such a comparison is not the correct first test to establish the soundness of the filtering approach. The rigor of the filtered equations approach should be established by comparing the predictions of the filtered model against the underlying microscopic two-fluid model. Only after establishing this fidelity should comparisons with experiments be undertaken, so that one can validate the microscopic two-fluid models (using the filtered equations to facilitate the computations).

Here we consider the first question that one would ask in establishing the usefulness of the filtered model equations. If the filtering process and the general form of the filtered constitutive models are meaningful, one would expect that the filtered model equations should afford considerably coarser structures than the microscopic two-fluid models would, even when we use very high spatial resolution in the simulations. We now demonstrate that this is indeed the case.

It is well known that according to the two-fluid model equations the state of uniform fluidization is most unstable to 1-D (vertical) disturbances that have no horizontal structure. Accordingly, we have focused our initial attention on a 1-D linear stability analysis (LSA). The 
LSA reveals that the state of uniform fluidization is stable to disturbances above a critical wavenumber where a Hopf bifurcation occurs. For wavenumbers smaller than this critical wavenumber $\left(k_{\mathrm{HB}}\right)$, unstable modes that take the form of traveling waves (that rise through the fluidized bed) obtain. Larger the value of $k_{H B}$, finer the structure of the inhomogeneities observed in the gas-particle suspension will be.

Our 1-D LSA with the filtered models revealed the following trend. In Figures 22a and $22 \mathrm{~b}$, it is shown how the growth rates of disturbances vary with the wave number of the disturbances for several filtered two-fluid models. The filtered two-fluid models, given in Table 3, were constructed for the curves presented in Figures $8,12 \mathrm{~b}$ and $13 \mathrm{~b}$. It is readily clear from the figures that the effective $k_{\text {Нв }}$ is a function of the filter size; in particular, the effective $k_{н в}$ decreased as the filter size increased. This is reassuring, as it shows that filtering is indeed erasing the fine structure and only presenting coarser structures. In addition, as the filter size increases the structures get progressively coarser. Thus, the filtering operation has indeed averages over the fine structures and generates equations and constitutive models that are suitable for integration over coarser grids.

\section{Summary}

We have presented a methodology where computational results obtained through highly resolved simulations (in a large periodic domain) of a given microscopic two-fluid model are filtered to deduce closures for the corresponding filtered two-fluid model equations. These filtered closures depend on the filter size and can readily be constructed for a range of filter sizes. We found that, to a good approximation, the dimensionless filtered drag coefficient, particle phase pressure and particle phase viscosity can be treated as functions of only particle volume 
fraction and dimensionless filter size. The effective drag coefficient to describe the inter-phase interaction force in the filtered equations shows two distinct regimes. At particle volume fractions greater than about 0.075 , it follows an effective Richardson-Zaki relationship and the effective R-Z exponent and apparent terminal velocity have an understandable physical interpretation in terms of interactions between particle clusters instead of the individual particles. At low particle volume fractions, the drag coefficient shows an anomalous behavior that is consistent with the formation of larger and denser clusters with increasing particle volume fraction.

The velocity fluctuations associated with the very complicated inhomogeneous structures shown by the microscopic two-fluid simulations dictate the magnitudes of the filtered particle phase pressure and viscosity. The contributions of the kinetic theory pressure and viscosity to these filtered quantities are negligibly small and so, for practically relevant filter sizes, one need not include the filtered granular energy equation in the analysis. This, however, does not mean that the fluctuations at the level of the individual particles, which the kinetic theory strives to model, are not important at all; these fluctuations influence the inhomogeneous microstructure and their velocity fluctuations, and hence the closures for the filtered equations.

The filtered two-fluid models based on seven filter lengths were compared in a 1D linear stability analysis. This analysis showed that filtering is indeed erasing the fine structure and only presenting coarser structures.

It is clear from our simulation results that there is a striking similarity between the 2-D and 3-D results. Although there are quantitative differences between 2-D and 3-D, the following characteristics were found to be common between them: 
a) The filtered drag coefficient decreased with increasing filter size, and

b) The filtered particle phase pressure and viscosity increased with filter size.

It seems reasonable to expect that the clusters will not grow beyond some critical size; if this is indeed the case, the filtered drag coefficient, and particle phase pressure and viscosity will become nearly independent of the filter size beyond some critical value. It is important to understand if such saturation occurs and, if so, at what filter size. It is also important to incorporate the effects of bounding walls on the filtered closures as comparison of the filtered model predictions with experimental data cannot be pursued until this issue is addressed. These are fertile problems for further research.

In the present study, we have absorbed the $-\overline{\phi^{\prime} \nabla p_{g}^{\prime}}$ term into the filtered the drag force. Zhang and VanderHeyden ${ }^{40}$ and de Wilde ${ }^{41,42}$ argue that $-\overline{\phi^{\prime} \nabla p_{g}^{\prime}}$ should also include a dynamic part (namely, an added mass force). Andrews ${ }^{43}$ found in his simulation study that the principal contribution of $-\overline{\phi^{\prime} \nabla p_{g}^{\prime}}$ was to a filtered drag force term, which we have included in our study. A more thorough investigation of the dynamic contribution would also be of interest.

\section{Acknowledgments}

This work was supported by the US Department of Energy (grants: CDE-FC2600NT40971 and DE-PS26-05NT42472-11) and the ExxonMobil Research \& Engineering Company. Andrews and Igci acknowledge summer training on MFIX at the National Energy Technology Laboratory, Morgantown, WV. We thank Madhava Syamlal, Chris Guenther, Ronald Breault and Sofiane Benyahia for their assistance throughout the course of this study. 


\section{Nomenclature}

$C_{D} \quad$ Single particle drag coefficient

$d \quad$ Particle diameter $(\mathrm{m})$

$e_{p} \quad$ Coefficient of restitution for particle-particle collisions

f Inter-phase interaction force per unit volume in the microscopic two-fluid model $\left(\mathrm{kg} / \mathrm{m}^{2} \cdot \mathrm{s}^{2}\right)$

$\overline{\mathbf{f}} \quad$ Filtered value of $\mathbf{f}\left(\mathrm{kg} / \mathrm{m}^{2} \cdot \mathrm{s}^{2}\right)$

$\overline{\mathbf{F}} \quad$ Inter-phase interaction force per unit volume in the filtered two-fluid model $\left(\mathrm{kg} / \mathrm{m}^{2} \cdot \mathrm{s}^{2}\right)$

$\mathrm{Fr}_{f} \quad$ Froude number based on filter size $=\mathrm{v}_{\mathrm{t}}^{2} / g \Delta_{f}$

$g, \mathbf{g} \quad$ Acceleration due to gravity $\left(\mathrm{m} / \mathrm{s}^{2}\right)$

$g_{0} \quad$ value of radial distribution function at contact (see expression in Table 1)

$G(\mathbf{x}, \mathbf{y}) \quad$ Weight function $\left(\mathrm{m}^{-3}\right)$

$\mathrm{J}_{\text {coll }} \quad$ Rate of dissipation of granular energy per unit volume by collisions between particles $\left(\mathrm{kg} / \mathrm{m} \cdot \mathrm{s}^{3}\right)$

$\mathrm{J}_{\mathrm{vis}} \quad$ Rate of dissipation of granular energy per unit volume by the relative motion between gas and particles $\left(\mathrm{kg} / \mathrm{m} \cdot \mathrm{s}^{3}\right)$

$N_{R Z, a p p} \quad$ Apparent Richardson-Zaki exponent

$p_{g}, p_{s} \quad$ Gas and particle phase pressures in the microscopic two-fluid model, respectively $\left(\mathrm{kg} / \mathrm{m} \cdot \mathrm{s}^{2}\right)$

$\overline{p_{g}}, \overline{p_{s}} \quad$ Filtered value of $p_{g}$ and $p_{s}$, respectively $\left(\mathrm{kg} / \mathrm{m} . \mathrm{s}^{2}\right)$

$\overline{p_{s, d}} \quad \overline{p_{s}}$ made dimensionless; $\overline{p_{s, d}}=\overline{p_{s}} / \rho_{s} \mathrm{v}_{\mathrm{t}}^{2}$

$\overline{p_{s e}} \quad$ Filtered particle phase pressure $\left(\mathrm{kg} / \mathrm{m} . \mathrm{s}^{2}\right)$

$\overline{p_{s e, d}} \quad \overline{p_{s e}}$ made dimensionless; $\overline{p_{s e, d}}=\overline{p_{s e}} / \rho_{s} \mathrm{v}_{\mathrm{t}}^{2}$

q Flux of granular energy $\left(\mathrm{kg} / \mathrm{s}^{3}\right)$

$\operatorname{Re}_{g} \quad$ Single particle Reynolds number 
T Granular temperature $\left(\mathrm{m}^{2} / \mathrm{s}^{2}\right)$

$\mathbf{u}, \mathbf{v}$ Gas and particle phase velocities in the microscopic two-fluid model, respectively $(\mathrm{m} / \mathrm{s})$

$\overline{\mathbf{u}}, \overline{\mathbf{v}} \quad$ Filtered gas and particle phase velocities, respectively $(\mathrm{m} / \mathrm{s})$

$\mathbf{u}^{\prime}, \mathbf{v}^{\prime} \quad$ Fluctuations in gas and particle phase velocities, respectively $(\mathrm{m} / \mathrm{s})$

$\mathrm{v}_{\mathrm{t}} \quad$ Terminal settling velocity $(\mathrm{m} / \mathrm{s})$

$V_{t, a p p} \quad$ Dimensionless apparent terminal velocity

$\mathbf{x}, \mathbf{y} \quad$ Position vectors (m)

\section{Greek Symbols}

$\beta \quad$ Drag coefficient in the microscopic two-fluid model $\left(\mathrm{kg} / \mathrm{m}^{3} . \mathrm{s}\right)$

$\overline{\beta_{e}} \quad$ Filtered drag coefficient $\left(\mathrm{kg} / \mathrm{m}^{3} . \mathrm{s}\right)$

$\overline{\beta_{e, d}} \quad$ Dimensionless filtered drag coefficient $=\overline{\beta_{e}} \mathrm{v}_{\mathrm{t}} / \rho_{s} g$

$\phi_{s}, \phi_{g} \quad$ Particle and gas phase volume fractions, respectively

$\overline{\phi_{s}}, \overline{\phi_{g}} \quad$ Filtered particle and gas phase volume fractions, respectively

$\phi_{s}^{\prime} \quad$ Fluctuation in particle phase volume fraction

$\left\langle\phi_{s}\right\rangle, \quad$ Domain-average particle volume fraction

$\phi_{\mathrm{s}, \max } \quad$ Maximum particle volume fraction

$\rho_{s}, \rho_{g} \quad$ Particle and gas densities, respectively $\left(\mathrm{kg} / \mathrm{m}^{3}\right)$

$\Delta_{f} \quad$ Filter size (m)

$\boldsymbol{\sigma}_{s}, \boldsymbol{\sigma}_{g} \quad$ Particle and gas phase stress tensors in the microscopic two-fluid model, respectively $\left(\mathrm{kg} / \mathrm{m} . \mathrm{s}^{2}\right)$

$\overline{\boldsymbol{\sigma}}_{s}, \overline{\boldsymbol{\sigma}}_{g} \quad$ Filtered values of $\boldsymbol{\sigma}_{s}$ and $\boldsymbol{\sigma}_{g}$, respectively $\left(\mathrm{kg} / \mathrm{m} . \mathrm{s}^{2}\right)$

$\Sigma_{s} \quad$ Filtered total particle phase stress $\left(\mathrm{kg} / \mathrm{m} . \mathrm{s}^{2}\right)$

$\Gamma_{\text {slip }} \quad$ Rate of generation of granular energy per unit volume by gas-particle slip $\left(\mathrm{kg} / \mathrm{m} \cdot \mathrm{s}^{3}\right)$ 
$\eta, \alpha, \lambda, \mu \quad$ Quantities defined in Table 1

$\lambda^{*}, \mu^{*} \quad$ Quantities defined in Table 1

$\lambda_{\mathrm{s}} \quad$ Granular thermal conductivity $(\mathrm{kg} / \mathrm{m} . \mathrm{s})$

$\mu_{g} \quad$ Gas phase viscosity $(\mathrm{kg} / \mathrm{m} . \mathrm{s})$

$\hat{\mu}_{g} \quad$ Effective gas phase viscosity appearing in the microscopic two-fluid model (taken to be equal to $\mu_{g}$ itself in our simulations) $(\mathrm{kg} / \mathrm{m} . \mathrm{s})$

$\mu_{b}, \mu_{s} \quad$ Bulk and shear viscosities of the particle phase appearing in the kinetic theory model $(\mathrm{kg} / \mathrm{m} . \mathrm{s})$

$\overline{\mu_{s, d}} \quad \mu_{s}$ made dimensionless; $\overline{\mu_{s, d}}=\overline{\mu_{s}} g / \rho_{s} \mathrm{v}_{\mathrm{t}}^{3}$

$\overline{\mu_{b e}}, \overline{\mu_{s e}} \quad$ Bulk and shear viscosities of the particle phase appearing in the filtered two-fluid model $(\mathrm{kg} / \mathrm{m} . \mathrm{s})$

$\overline{\mu_{s e, d}} \quad \overline{\mu_{s e}}$ made dimensionless; $\overline{\mu_{s e, d}}=\overline{\mu_{s e}} g / \rho_{s} \mathrm{v}_{\mathrm{t}}^{3}$ 


\section{References Cited}

1) Grace JR, Bi H. in Circulating Fluidized Beds, JR Grace, AA Avidan and TM Knowlton (eds.), Blackie Academic \& Professional, New York (1997);1.

2) Gidaspow D. Multiphase Flow and Fluidization, Academic Press, CA.; 1994.

3) Jackson R. The dynamics of fluidized particles. Cambridge University Press; 2000.

4) Syamlal M, Rogers W, O'Brien TJ. MFIX Documentation. U.S. Department of Energy, Federal Energy Technology Center: Morgantown, WV; 1993.

5) Syamlal M. MFIX Documentation: Numerical Techniques. DOE/MC-31346-5824. NTIS/DE98002029; 1998. Also see www.mfix.org.

6) Wen CY, Yu YH. Mechanics of fluidization. Chem. Eng. Prog. Symp. Ser. 1966;62: 100111.

7) Ergun S. Fluid flow through packed columns. Chem. Eng. Prog. 1952;48:89.

8) Hill RJ, Koch DL, Ladd AJC. The first effects of fluid inertia on flow in ordered and random arrays of spheres. J. Fluid. Mech. 2001;448:213-241.

9) Hill RJ, Koch DL, Ladd AJC. Moderate-Reynolds-number flows in ordered and random arrays of spheres. J. Fluid Mech. 2001;448:243-278.

10) Wylie JJ, Koch DL, Ladd AJC. Rheology of suspensions with high particle inertia and moderate fluid inertia. J. Fluid Mech. 2003;480:95-118.

11) Khandai D, Derksen JJ, van den Akker HEA. Interphase drag coefficients in gas-solid flows. AIChE J. 2003;49:1060.

12) Li J, Kuipers JAM. Gas-particle interactions in dense gas-fluidized beds. Chem. Eng. Sci. 2003;58:711-718. 
13) van der Hoef MA, Boetstra R, Kuipers, JAM. Lattice Boltzmann simulations of low Reynolds number flow past mono- and bi-disperse arrays of spheres: results for the permeability and drag forces. J. Fluid Mech. 2005;528:233-254.

14) Benyahia S, Syamlal M, O’Brien TJ. Extension of Hill-Koch-Ladd drag correlation over all ranges of Reynolds number and solids volume fraction. Powder Technol. 2006;162:166-174.

15) Gidaspow D, Jung J, Singh RJ. Hydrodynamics of fluidization using kinetic theory: An emerging paradigm. 2002 Fluor-Daniel Lecture. Powder Technol. 2004;148:123-141.

16) Koch DL, Sangani AS. Particle pressure and marginal stability limits for a homogeneous monodisperse gas fluidized bed: kinetic theory and numerical simulations. J. Fluid Mech. 1999;400:229-263.

17) Huilin L, Yurong H, Gidaspow D, Lidan Y. Yukun Q. Size segregation of binary mixture of solids in bubbling fluidized beds. Powder Technol. 2003;134:86-97.

18) Iddir H, Arastoopour H, Hrenya C M. Analysis of binary and ternary granular mixtures behavior using the kinetic theory approach. Powder Technol. 2005;151:117-125.

19) Arnarson BO, Jenkins JT. Binary mixtures of inelastic spheres: Simplified constitutive theory. Phys. Fluids 2004;16:4543-4550.

20) Jenkins J, Mancini F. Kinetic theory for binary mixtures of smooth, nearly elastic spheres. Phys. Fluids A 1989;1:2050.

21) Benyahia S, Arastoopour H, Knowlton TM. Simulation of particles and gas flow behavior in the riser section of a circulating fluidized bed using the kinetic theory approach for the particulate phase. Powder Technol. 2000;112:24-33. 
22) Ding J, Gidaspow D. A Bubbling fluidization model using kinetic-theory of granular flow. AIChE J. 1990;36:523-538.

23) Goldschmidt MJV, Kuipers JAM. van Swaaij WPM. Hydrodynamic modeling of dense gas-fluidized beds using the kinetic theory of granular flow: Effect of restitution coefficient on bed dynamics. Chem. Eng. Sci.,2001;56:571-578.

24) Neri A, Gidaspow D. Riser hydrodynamics: Simulation using kinetic theory. AIChE J. 2000;46:52-67.

25) Lun CKK, Savage SB, Jeffrey DJ, Chepurniy N. Kinetic theories of granular flows: inelastic particles in Couette flow and slightly inelastic particles in a general flow field. $J$. Fluid Mech. 1984;140:223-256.

26) Sinclair JL, Jackson R. Gas-particle flow in a vertical pipe with particle-particle interaction. AIChE J. 1989;35:1473-1486.

27) Pita JA, Sundaresan S. Gas-solid flow in vertical tubes. AIChE J. 1991;37:1009-1018.

28) Pita JA, Sundaresan S. Developing flow of a gas-particle mixture in a vertical riser. AIChE J. 1993;39:541-552.

29) Louge M, Mastorakos E, Jenkins JT. The role of particle collisions in pneumatic transport. J. Fluid Mech. 1991;231:345-359.

30) Agrawal K, Loezos PN, Syamlal M, Sundaresan S. The Role of meso-scale structures in rapid gas-solid flows. J. Fluid Mech. 2001;445:151-185.

31) Andrews AT IV, Loezos PN, Sundaresan S. Coarse-grid simulation of gas-particle flows in vertical risers. Ind. Eng. Chem. Res. 2005;44:6022-6037.

32) Sundaresan S. Perspective: Modeling the hydrodynamics of multiphase flow reactors: Current status and challenges. AIChE J. 2000;46:1102-1105. 
33) O'Brien TJ, Syamlal M. Particle cluster effects in the numerical simulation of a circulating fluidized bed. In Circulating Fluidized Bed Technology IV, A Avidan (ed), Proceedings of the Fourth International Conference on Circulating Fluidized Beds, Hidden Valley Conference Center and Mountain Resort, Somerset, PA, August 1-5, 1993.

34) Boemer A, Qi H, Hannes J, Renz U. Modelling of solids circulation in a fluidised bed with Eulerian approach. 29th IEA-FBC Meeting in Paris, France, Nov. 24-26, 1994.

35) Heynderickx GJ, Das AK, de Wilde J, Marin GB. Effect of clustering on gas-solid drag in dilute two-phase flow. Ind. Eng. Chem. Res. 2004;43:4635.

36) McKeen T, Pugsley T. Simulation and experimental validation of a freely bubbling bed of FCC catalyst. Powder Technol. 2003;129:139-152.

37) Yang N, Wang W, Ge W, Wang L. Li, J. Simulation of heterogeneous structure in a circulating fluidized bed riser by combining the two-fluid model with EMMS approach. Ind. Eng. Chem. Res. 2004;43:5548-5561.

38) Dasgupta S, Jackson R, Sundaresan S. Turbulent gas-particle flow in vertical risers. AIChE J. 1994;40:215-228.

39) Hrenya CM, Sinclair JL. Effects of particle-phase turbulence in gas-solid flows. AIChE J. 1997;43:853-869.

40) Zhang DZ, VanderHeyden WB. The effects of mesoscale structures on the macroscopic momentum equations for two-phase flows. Int. J. Multiphase Flow 2002;28:805-822.

41) De Wilde J. Reformulating and quantifying the generalized added mass in filtered gassolid flow models. Phys. Fluids 2005;17:113304:1-14.

42) De Wilde J. The generalized added mass revised. Phys. Fluids 2007;19:058103:1-4. 
43) Andrews AT IV. Filtered models for gas-particle hydrodynamics. PhD Dissertation, Princeton University, Princeton, NJ, USA. 2007.

44) Richardson JF, Zaki WN. Sedimentation and fluidization: Part I. Trans. Inst. Chem. Engrs. 1954;32:35-53. 
Table 1: Model equations for gas-particle flows

$$
\begin{gathered}
\frac{\partial \rho_{\mathrm{s}} \phi_{s}}{\partial \mathrm{t}}+\nabla \cdot\left(\rho_{\mathrm{s}} \phi_{s} \mathbf{v}\right)=0 \\
\frac{\partial\left(\rho_{\mathrm{g}}\left(1-\phi_{s}\right)\right)}{\partial \mathrm{t}}+\nabla \cdot\left[\rho_{\mathrm{g}}\left(1-\phi_{s}\right) \mathbf{u}\right]=0 \\
{\left[\frac{\partial\left(\rho_{\mathrm{s}} \phi_{\mathrm{s}} \mathbf{v}\right.}{\partial \mathrm{t}}+\nabla \cdot\left(\rho_{\mathrm{s}} \phi_{s} \mathbf{v v}\right)\right]=-\nabla \cdot \boldsymbol{\sigma}_{s}-\phi_{s} \nabla \cdot \boldsymbol{\sigma}_{g}+\mathbf{f}+\rho_{\mathrm{s}} \phi_{s} \mathbf{g}} \\
{\left[\frac{\partial\left(\rho_{g}\left(1-\phi_{s}\right) \mathbf{u}\right)}{\partial \mathrm{t}}+\nabla \cdot\left(\rho_{g}\left(1-\phi_{s}\right) \mathbf{u u}\right)\right]=-\left(1-\phi_{s}\right) \nabla \cdot \boldsymbol{\sigma}_{g}-\mathbf{f}+\rho_{g}\left(1-\phi_{s}\right) \mathbf{g}} \\
{\left[\frac{\partial\left(\frac{3}{2} \rho_{s} \phi_{s} \mathrm{~T}\right)}{\partial \mathrm{t}}+\nabla \cdot\left(\frac{3}{2} \rho_{s} \phi_{s} \mathrm{~T} \mathbf{v}\right)\right.} \\
{\left[\frac{\mathbf{f}}{2}\right]=-\nabla \cdot \mathbf{q}-\boldsymbol{\sigma}_{s}: \nabla \mathbf{v}+\Gamma_{\text {slip }}-\mathrm{J}_{\mathrm{coll}}-\mathrm{J}_{\mathrm{vis}}}
\end{gathered}
$$

\section{Gas phase stress tensor}

$$
\boldsymbol{\sigma}_{g}=p_{g} \mathbf{I}-\hat{\mu}_{g}\left[\nabla \mathbf{u}+(\nabla \mathbf{u})^{\mathrm{T}}-\frac{2}{3}(\nabla \cdot \mathbf{u}) \mathbf{I}\right]
$$

\section{Gas-Particle Drag (Wen \& $\left.\mathbf{Y u}^{6}\right)$}

$$
\begin{gathered}
\mathbf{f}=\beta(\mathbf{u}-\mathbf{v}) ; \beta=\frac{3}{4} C_{D} \frac{\rho_{g}\left(1-\phi_{s}\right) \phi_{s}|\mathbf{u}-\mathbf{v}|}{d}\left(1-\phi_{s}\right)^{-2.65} \\
\mathrm{C}_{\mathrm{D}}=\left\{\begin{array}{cc}
\frac{24}{\operatorname{Re}_{g}}\left(1+0.15 \operatorname{Re}_{g}^{0.687}\right) & \operatorname{Re}_{g}<1000 \\
0.44 & \operatorname{Re}_{g} \geq 1000
\end{array} \operatorname{Re}_{g}=\frac{\left(1-\phi_{s}\right) \rho_{g} d|\mathbf{u}-\mathbf{v}|}{\mu_{g}}\right.
\end{gathered}
$$




\section{Table 1 - continued}

Kinetic theory model for particle phase stress

$$
\boldsymbol{\sigma}_{s}=\left[p_{s}-\eta \mu_{b}(\nabla \cdot \mathbf{v})\right] \mathbf{I}-2 \mu_{s} \mathbf{S}
$$

where

$$
p_{s}=\rho_{s} \phi_{s}\left(1+4 \eta \phi_{s} g_{o}\right) \mathrm{T}, \mathbf{S}=\frac{1}{2}\left(\nabla \mathbf{v}+(\nabla \mathbf{v})^{\mathrm{T}}\right)-\frac{1}{3}(\nabla \cdot \mathbf{v}) \mathbf{I}
$$

$$
\mu_{s}=\left(\frac{2+\alpha}{3}\right)\left\{\frac{\mu^{*}}{g_{o} \eta(2-\eta)}\left(1+\frac{8}{5} \phi_{s} \eta g_{o}\right)\left(1+\frac{8}{5} \eta(3 \eta-2) \phi_{s} g_{o}\right)+\frac{3}{5} \eta \mu_{b}\right\}
$$

$$
\begin{gathered}
\mu^{*}=\frac{\mu}{1+\frac{2 \beta \mu}{\left(\rho_{s} \phi_{s}\right)^{2} g_{o} \mathrm{~T}}} ; \mu=\frac{5 \rho_{s} d \sqrt{\pi \mathrm{T}}}{96} ; \\
\mu_{b}=\frac{256 \mu \phi_{s}^{2} g_{o}}{5 \pi} ; \eta=\frac{\left(1+e_{p}\right)}{2} ; g_{o}=\frac{1}{1-\left(\phi_{s} / \phi_{s, \max }\right)^{1 / 3}} ; \phi_{\mathrm{s}, \max }=0.65 ; \alpha=1.6
\end{gathered}
$$

\section{$\underline{\text { Kinetic theory model for pseudo-thermal energy flux }}$}

$$
\mathbf{q}=-\lambda_{s} \nabla \mathrm{T}
$$

$$
\begin{gathered}
\text { where } \lambda_{s}=\frac{\lambda^{*}}{g_{o}}\left\{\left(1+\frac{12}{5} \eta \phi_{s} g_{o}\right)\left(1+\frac{12}{5} \eta^{2}(4 \eta-3) \phi_{s} g_{o}\right)+\frac{64}{25 \pi}(41-33 \eta) \eta^{2} \phi_{s}^{2} g_{o}^{2}\right\} \\
\lambda^{*}=\frac{\lambda}{1+\frac{6 \beta \lambda}{5\left(\rho_{s} \phi_{s}\right)^{2} g_{o} \mathrm{~T}}} ; \lambda=\frac{75 \rho_{s} d \sqrt{\pi \mathrm{T}}}{48 \eta(41-33 \eta)}
\end{gathered}
$$




\section{Table 1 - continued}

Kinetic theory model for rate of dissipation of pseudo-thermal energy through collisions

$$
\mathrm{J}_{\text {coll }}=\frac{48}{\sqrt{\pi}} \eta(1-\eta) \frac{\rho_{s} \phi_{s}^{2}}{\mathrm{~d}} g_{o} \mathrm{~T}^{3 / 2}
$$

\section{Effect of fluid on particle phase fluctuation energy (Koch \& Sangani ${ }^{16}$ )}

$$
\begin{aligned}
& \mathrm{J}_{\text {vis }}=\frac{54 \phi_{s} \mu_{\mathrm{g}} \mathrm{T}}{d^{2}} \mathrm{R}_{\text {diss }} \text {, where } \\
& \mathrm{R}_{\mathrm{diss}}=1+\frac{3 \phi_{\mathrm{s}}^{1 / 2}}{\sqrt{2}}+\frac{135}{64} \phi_{s} \ln \phi_{\mathrm{s}} \\
& +11.26 \phi_{s}\left(1-5.1 \phi_{s}+16.57 \phi_{s}^{2}-21.77 \phi_{s}^{3}\right)-\phi_{s} g_{0} \ln (0.01) \\
& \Gamma_{\text {slip }}=\frac{81 \phi_{s} \mu_{\mathrm{g}}^{2}|\mathbf{u}-\mathbf{v}|}{g_{\mathrm{o}} d^{3} \rho_{\mathrm{s}} \sqrt{\pi \mathrm{T}}} \Psi, \quad \text { where } \\
& \Psi=\frac{\mathrm{R}_{\mathrm{d}}^{2}}{\left(1+3.5 \phi_{s}^{1 / 2}+5.9 \phi_{s}\right)}, \\
& \mathrm{R}_{\mathrm{d}}=\left\{\begin{array}{l}
\frac{1+3\left(\phi_{s} / 2\right)^{1 / 2}+(135 / 64) \phi_{s} \ln \phi_{s}+17.14 \phi_{s}}{1+0.681 \phi_{s}-8.48 \phi_{s}^{2}+8.16 \phi_{s}^{3}}, \phi_{s}<0.4 \\
\frac{10 \phi_{s}}{\left(1-\phi_{s}\right)^{3}}+0.7, \phi_{s} \geq 0.4
\end{array}\right.
\end{aligned}
$$


Table 2: Physical properties of gas and solids

\begin{tabular}{lll}
\hline & particle diameter & $7.5 \times 10^{-6} \mathrm{~m}$ \\
$\rho_{\mathrm{s}}$ & particle density & $1500 \mathrm{~kg} / \mathrm{m}^{3}$ \\
$\rho_{\mathrm{g}}$ & Gas density & $1.3 \mathrm{~kg} / \mathrm{m}^{3}$ \\
$\mu_{\mathrm{g}}$ & Gas viscosity & $1.8 \times 10^{-5} \mathrm{~kg} / \mathrm{m} \cdot \mathrm{s}$ \\
$e_{p}$ & Coefficient of restitution & 0.9 \\
$\mathrm{v}_{\mathrm{t}}$ & Terminal settling velocity & $0.2184 \mathrm{~m} / \mathrm{s}$ \\
$\frac{\mathrm{v}_{\mathrm{t}}^{2}}{\mathrm{~g}}$ & Characteristic length & $0.00487 \mathrm{~m}$ \\
$\frac{\mathrm{v}_{\mathrm{t}}}{\mathrm{g}}$ & Characteristic time & \\
$\rho_{\mathrm{s}} \mathrm{v}_{\mathrm{t}}^{2}$ & Characteristic stress & $71.55 \mathrm{~kg} / \mathrm{m} . \mathrm{s}^{2}$ \\
\hline
\end{tabular}


Filter region size: $\mathrm{Fr}_{f}^{-1}=g \Delta_{f} / \mathrm{v}_{t}^{2}=1.028$

$\frac{\bar{\beta}_{e} \mathrm{v}_{\mathrm{t}}}{\rho_{s} \phi_{s}\left(1-\phi_{s}\right) g}= \begin{cases}\frac{3.10296\left(1-\phi_{s}\right)^{3}-17.4801\left(1-\phi_{s}\right)^{3}+34.36\left(1-\phi_{s}\right)^{2}-28.7\left(1-\phi_{s}\right)+8.717}{\left(1-\phi_{s}\right)^{2}-2.0019\left(1-\phi_{s}\right)+1.002}, \phi_{s} \leq 0.04591 \\ \frac{1}{1.4356\left(1-\phi_{s}\right)^{3.513}}, & \phi_{s}>0.04591\end{cases}$

$\frac{\overline{p_{s e}}}{\rho_{s} \mathrm{v}_{\mathrm{t}}^{2}}=0.36 \phi_{s}\left(1-1.80 \phi_{s}\right), \quad \phi_{\mathrm{s}} \leq 0.30$

$\frac{\overline{\mu_{s e}} g}{\rho_{s} \mathrm{v}_{\mathrm{t}}^{3}}=0.24 \phi_{s}\left(1-0.86 \phi_{s}\right), \quad \phi_{\mathrm{s}} \leq 0.30$

Filter region size: $\mathrm{Fr}_{f}^{-1}=g \Delta_{f} / \mathrm{v}_{t}^{2}=2.056$

$\frac{\bar{\beta}_{e} \mathrm{v}_{\mathrm{t}}}{\rho_{s} \phi_{s}\left(1-\phi_{s}\right) g}=\left\{\begin{array}{l}\frac{4.227\left(1-\phi_{s}\right)^{4}-2.363\left(1-\phi_{s}\right)^{3}-4.324\left(1-\phi_{s}\right)^{2}-2.474\left(1-\phi_{s}\right)+4.904}{\left(1-\phi_{s}\right)^{2}-5.411\left(1-\phi_{s}\right)+4.427}, \phi_{s} \leq 0.040828 \\ \frac{1}{2.0747\left(1-\phi_{s}\right)^{3.574}}, \quad \phi_{s}>0.040828\end{array}\right.$

$\frac{\overline{p_{s e}}}{\rho_{s} \mathrm{v}_{\mathrm{t}}^{2}}=0.90 \phi_{s}\left(1-1.80 \phi_{s}\right), \quad \phi_{\mathrm{s}} \leq 0.30$

$\frac{\overline{\mu_{s e}} g}{\rho_{s} \mathrm{v}_{\mathrm{t}}^{3}}=\left\{0.60 \phi_{s}\left(1-0.86 \phi_{s}\right), \quad \phi_{\mathrm{s}} \leq 0.30\right.$ 
Filter region size: $\mathrm{Fr}_{f}^{-1}=g \Delta_{f} / \mathrm{v}_{t}^{2}=4.112$

$\frac{\overline{\beta_{e} v_{t}}}{\rho_{s} \phi_{s}\left(1-\phi_{s}\right) g}=\left\{\begin{array}{l}\frac{-1.779\left(1-\phi_{s}\right)^{4}+9.361\left(1-\phi_{s}\right)^{3}-4.042\left(1-\phi_{s}\right)^{2}-13.97\left(1-\phi_{s}\right)+10.44}{\left(1-\phi_{s}\right)^{2}-6.664\left(1-\phi_{s}\right)+5.676}, \phi_{s} \leq 0.072785 \\ \frac{1}{3.0192\left(1-\phi_{s}\right)^{3.929}}, \quad \phi_{s}>0.072785\end{array}\right.$

$\frac{\overline{p_{s e}}}{\rho_{s} \mathrm{v}_{\mathrm{t}}^{2}}=2.02 \phi_{s}\left(1-1.80 \phi_{s}\right), \quad \phi_{\mathrm{s}} \leq 0.30$

$\frac{\overline{\mu_{s e}} g}{\rho_{s} \mathrm{v}_{\mathrm{t}}^{3}}=1.40 \phi_{s}\left(1-0.86 \phi_{s}\right), \quad \phi_{\mathrm{s}} \leq 0.30$

Filter region size: $\mathrm{Fr}_{f}^{-1}=g \Delta_{f} / \mathrm{v}_{t}^{2}=8.224$

$\frac{\overline{\beta_{e}} \mathrm{v}_{\mathrm{t}}}{\rho_{s} \phi_{s}\left(1-\phi_{s}\right) g}=\left\{\begin{array}{l}\frac{42.7\left(1-\phi_{s}\right)^{4}-102.3\left(1-\phi_{s}\right)^{3}+93.35\left(1-\phi_{s}\right)^{2}-55.93\left(1-\phi_{s}\right)+22.2}{\left(1-\phi_{s}\right)^{2}-27.17\left(1-\phi_{s}\right)+26.2}, \phi_{s} \leq 0.015065 \\ \frac{1}{3.9709\left(1-\phi_{s}\right)^{4.112}}, \quad \phi_{s}>0.015065\end{array}\right.$

$\frac{\overline{p_{s e}}}{\rho_{s} \mathrm{v}_{\mathrm{t}}^{2}}=3.95 \phi_{s}\left(1-1.80 \phi_{s}\right), \quad \phi_{\mathrm{s}} \leq 0.30$

$\frac{\overline{\mu_{s e}} g}{\rho_{s} \mathrm{v}_{\mathrm{t}}^{3}}=3.28 \phi_{s}\left(1-0.86 \phi_{s}\right), \quad \phi_{\mathrm{s}} \leq 0.30$ 


\section{Figures:}
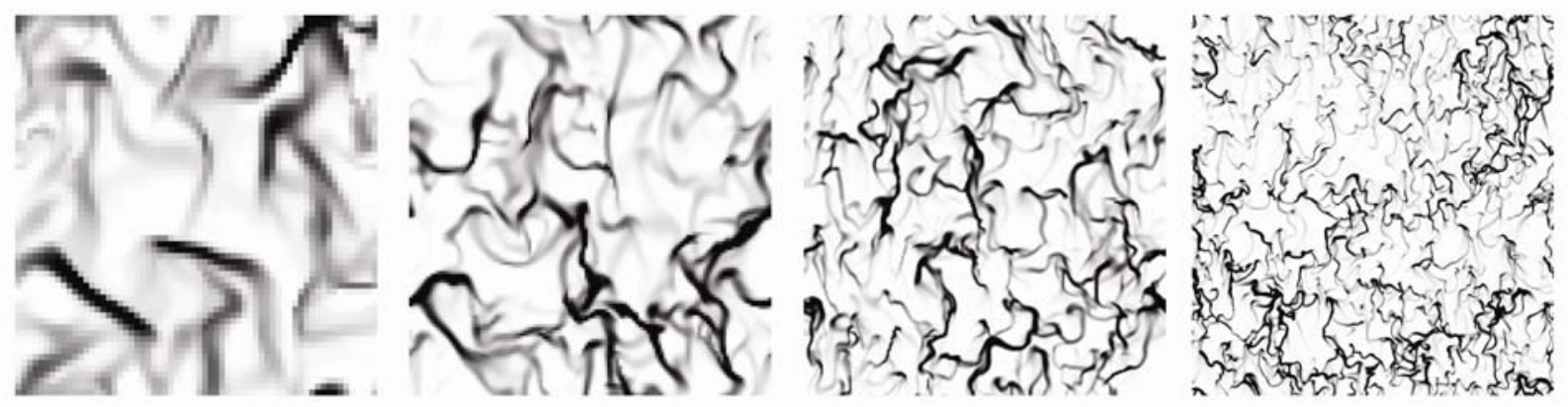

Figure 1: Snapshots of the particle volume fraction field in a large periodic domain of size $131.584 \mathrm{x}$ 131.584 dimensionless units are displayed. The physical conditions corresponding to this simulation are listed in Table 2. The domain-average particle volume fraction, $\left\langle\phi_{s}\right\rangle=0.05$. Simulations were performed with different resolutions: (a) $64 \times 64$ grids; (b) $128 \times 128$ grids; (c) $256 \times 256$ grids; (d) $512 \times 512$ grids. The gray scale axis ranges from $\phi_{s}=0.0$ (white) to $\phi_{s}=0.25$ (black). 


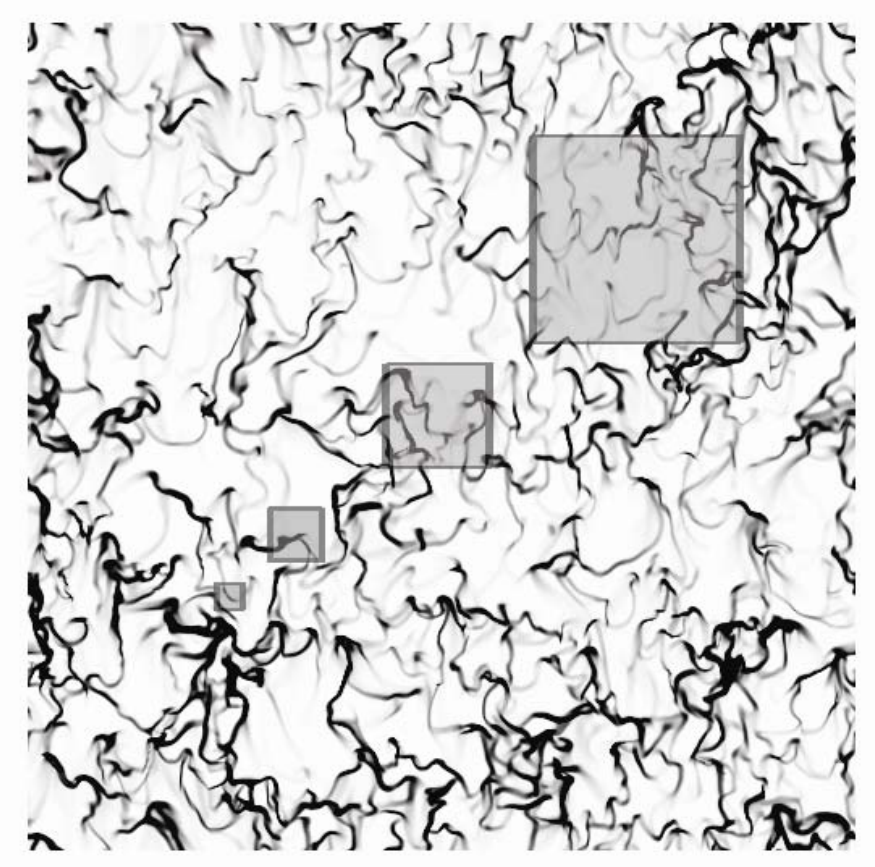

Figure 2: Snapshot of the particle volume fraction field in a large periodic domain of size $131.584 \mathrm{x}$ 131.584 dimensionless units are displayed. Simulations were performed with 512 x 512 grid points. Overlaid is a pictorial representation of region averaging, where regions of varying size are isolated and treated as individual realizations. Regions (filters) having dimensionless lengths of 4.112, 8.224, 16.448, and 32.896 are shown as shaded subsections. 


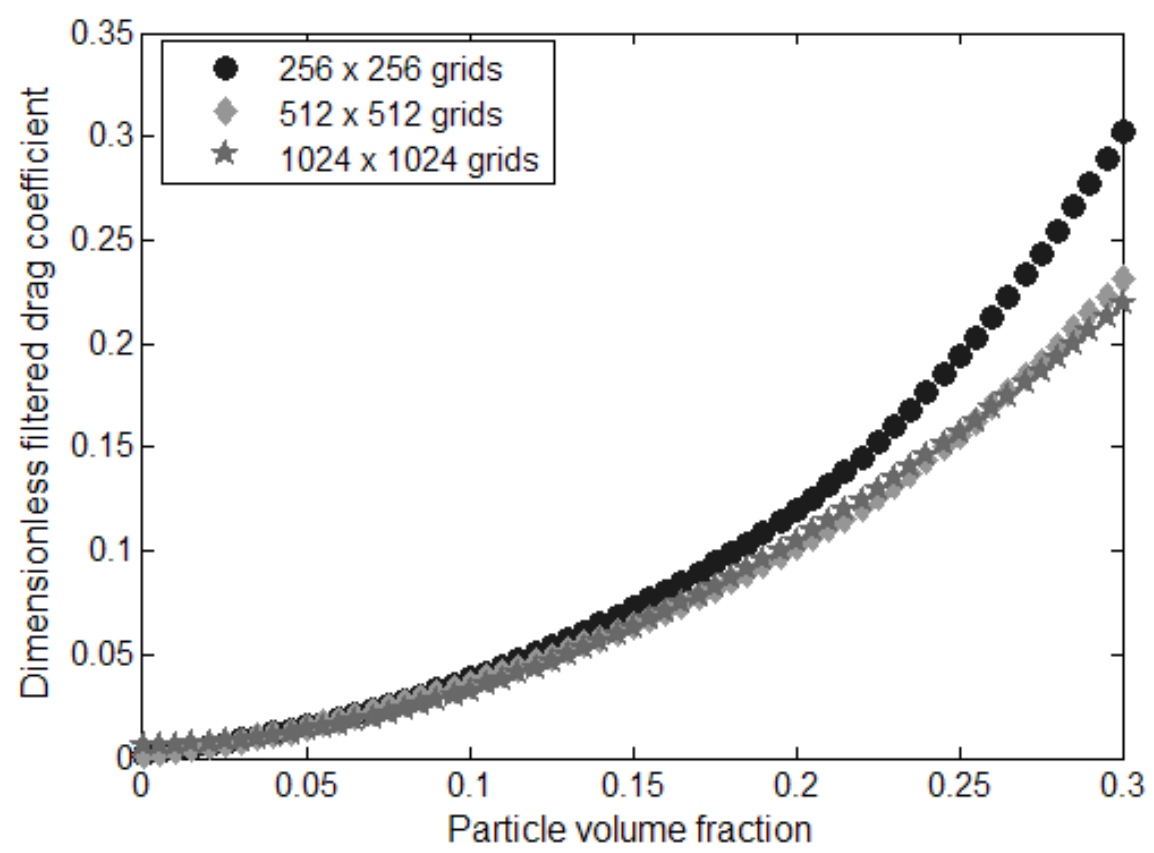

Figure 3: The variation of the dimensionless filtered drag coefficient with particle volume fraction, determined by filtering the computational data gathered from simulations in a large periodic domain of size $131.584 \times 131.584$ dimensionless units, is presented. The dimensionless filter length $=8.224$. The filtered drag coefficient includes contributions from the drag force and the pressure fluctuation force. Data used for filtering were generated by running simulations for domain-average particle volume fractions of $0.05,0.15,0.25$, and 0.35 . The figure shows results obtained by filtering data generated at different grid resolutions as marked in the legend. The top curve corresponds to result obtained with $256 \mathrm{x}$ 256 grids. 


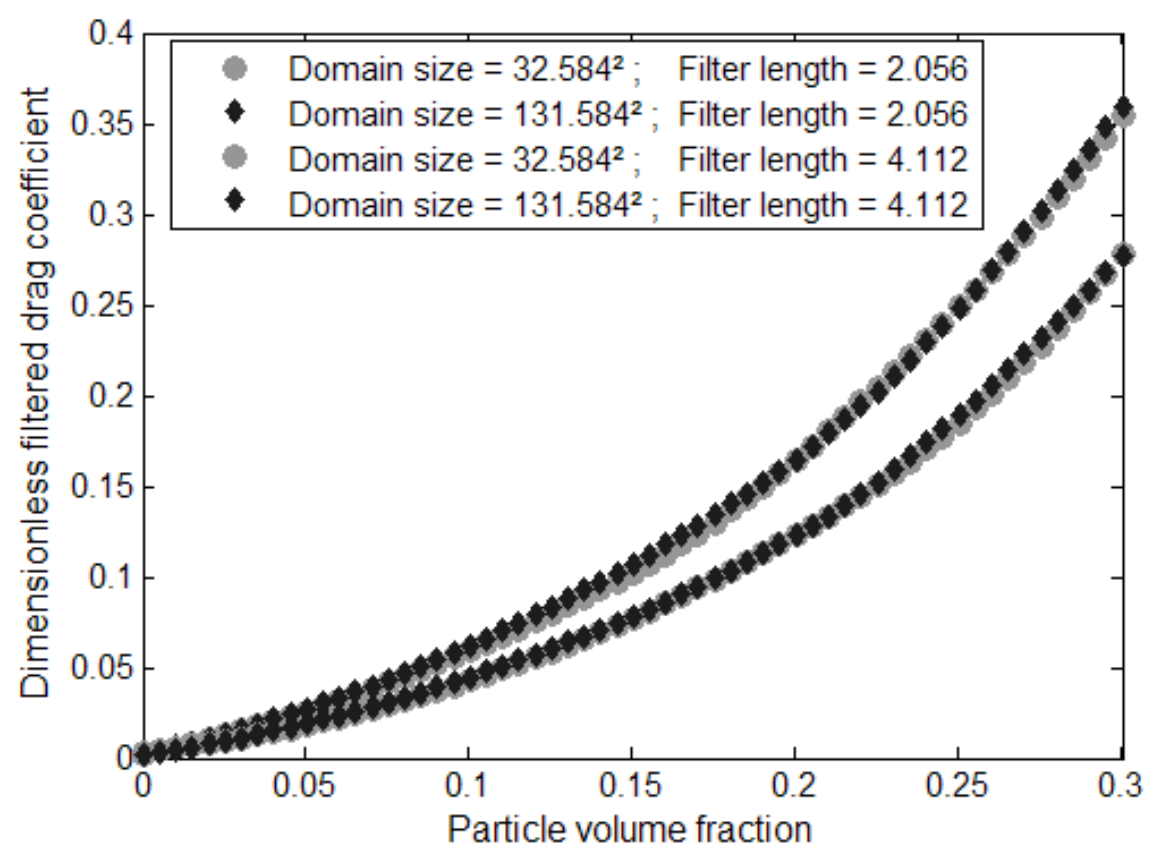

Figure 4: The effect of domain size on the dimensionless filtered drag coefficient is displayed. Data used for filtering were generated by running simulations at domain-average particle volume fractions of 0.02 , $0.05,0.10,0.15,0.20,0.25$, and 0.35 for two different square periodic domains of sizes: $131.584 \mathrm{x}$ 131.584 dimensionless units $(512 \times 512$ grids $)$ and $32.896 \times 32.896$ dimensionless units $(128 \times 128$ grids). The top two curves correspond to a dimensionless filter length of 2.056 , while the bottom two are for a dimensionless filter length of 4.112 . 


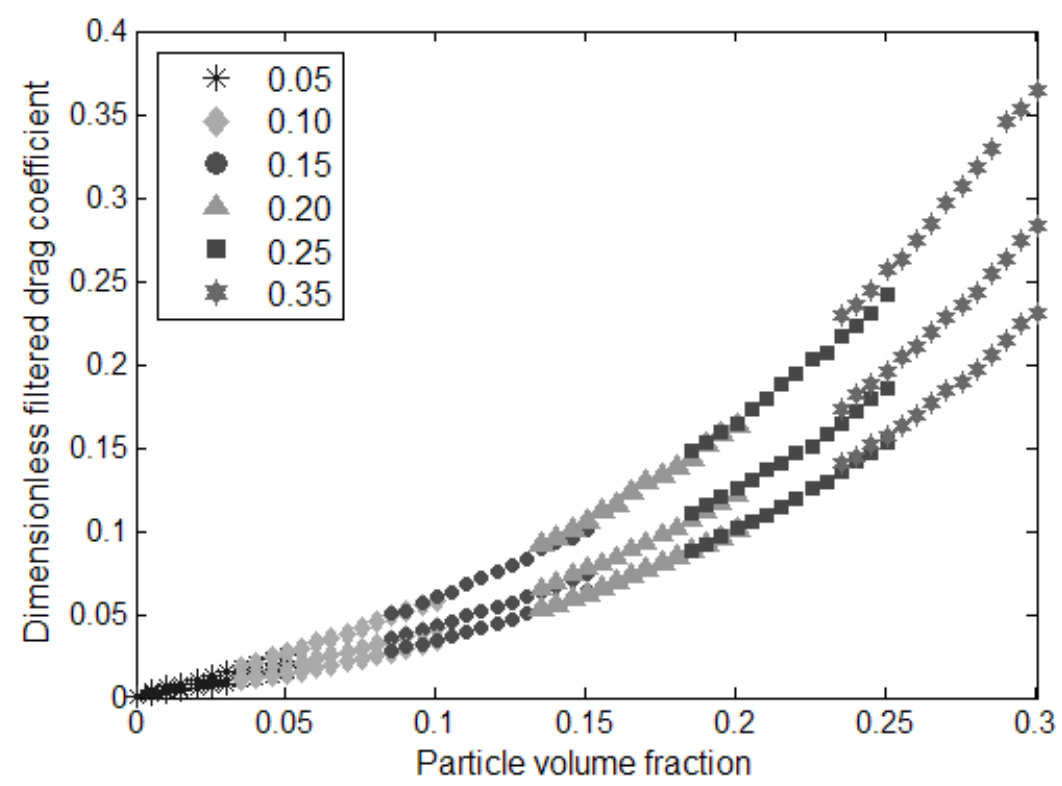

Figure 5: The effect of domain-average particle volume fraction on the dimensionless filtered drag coefficient is displayed. Simulations were performed in a square domain of size $131.584 \mathrm{x} 131.584$ dimensionless units and 512 × 512 grid points and domain-average particle volume fractions of 0.05 , $0.10,0.15,0.20,0.25$, and 0.35 (shown by different symbols in each curve). Results are presented for dimensionless filter lengths of 2.056 (top curve), 4.112 (middle curve) and 8.224 (bottom curve). 


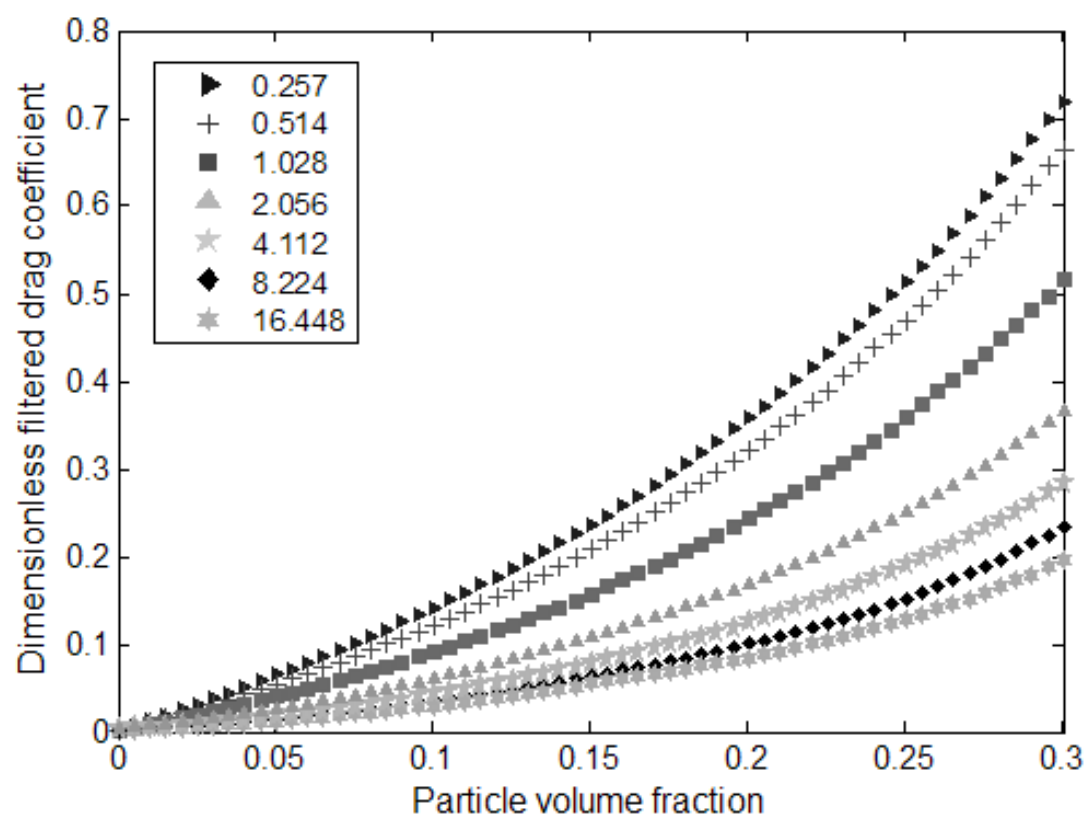

Figure 6a: The variation of the dimensionless filtered drag coefficient with particle volume fraction for various filter sizes (listed in the legend in dimensionless units) is shown. Simulations were performed in a square periodic domain of size 131.584 x 131.584 dimensionless units and using 512 x 512 grid points. Data used for filtering were generated by running simulations for domain-average particle volume fractions of $0.01,0.02,0.03,0.04,0.05,0.10,0.15,0.20,0.25,0.30$ and 0.35 . The dimensionless filter lengths are shown in the legend. 


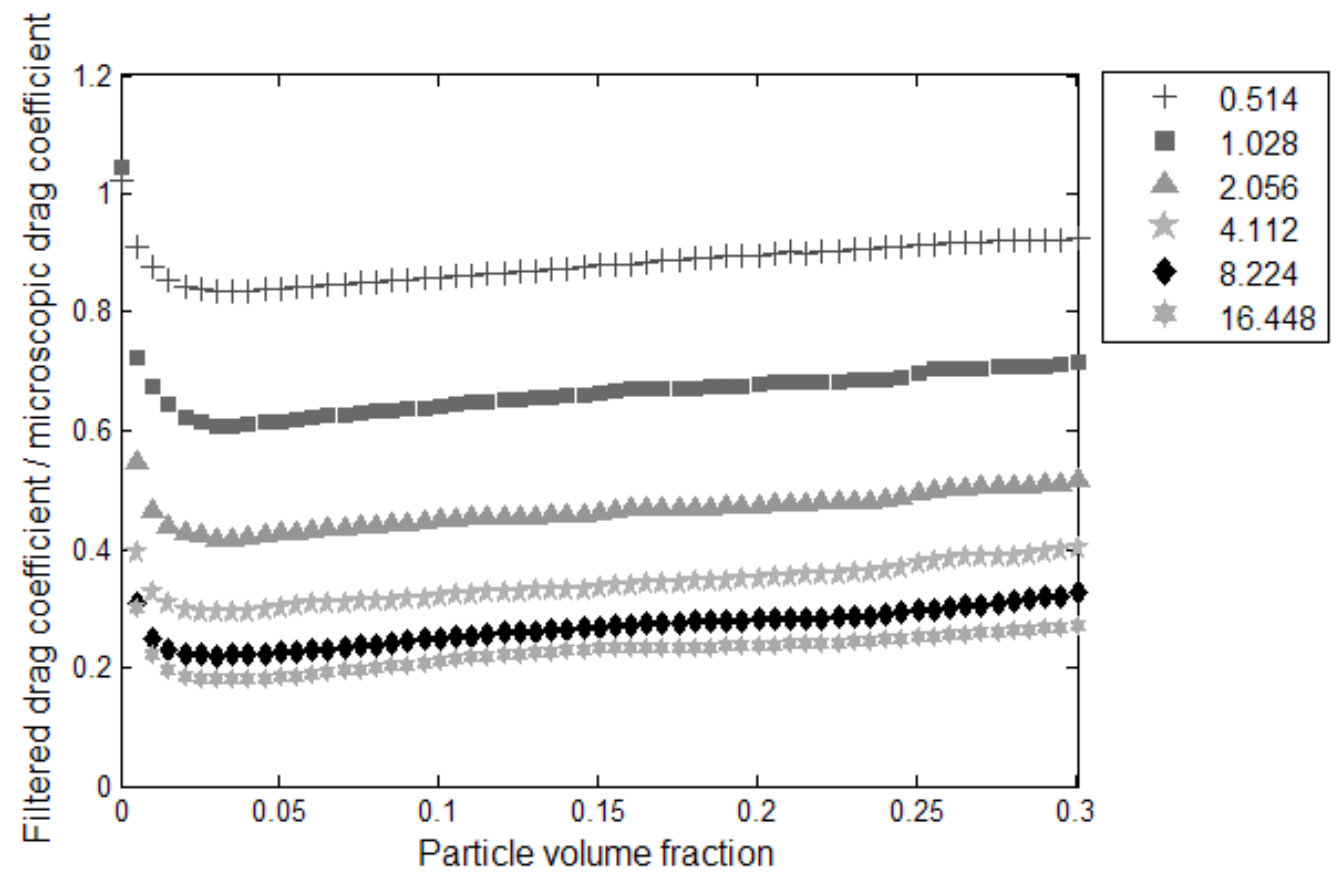

Figure 6b: An alternative representation of the filtered drag coefficient. The variation of the dimensionless filtered drag coefficient with particle volume fraction for various filter sizes (listed in the legend in dimensionless units) is shown. All conditions are as in Figure 6a. 


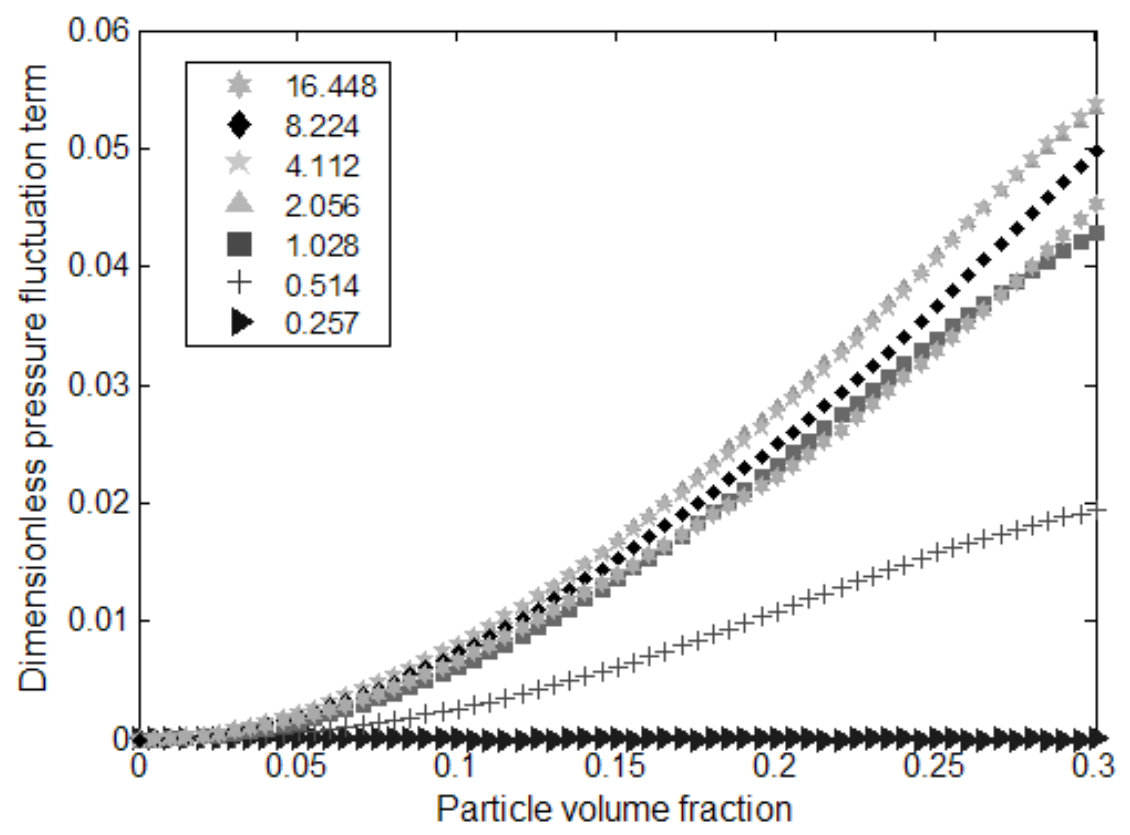

Figure 7: The contribution of the (dimensionless) pressure fluctuation term to the dimensionless filtered drag coefficient shown earlier in Figure 6a is presented. All conditions are as in Figure 6a. The dimensionless filter lengths are shown in the legend. 


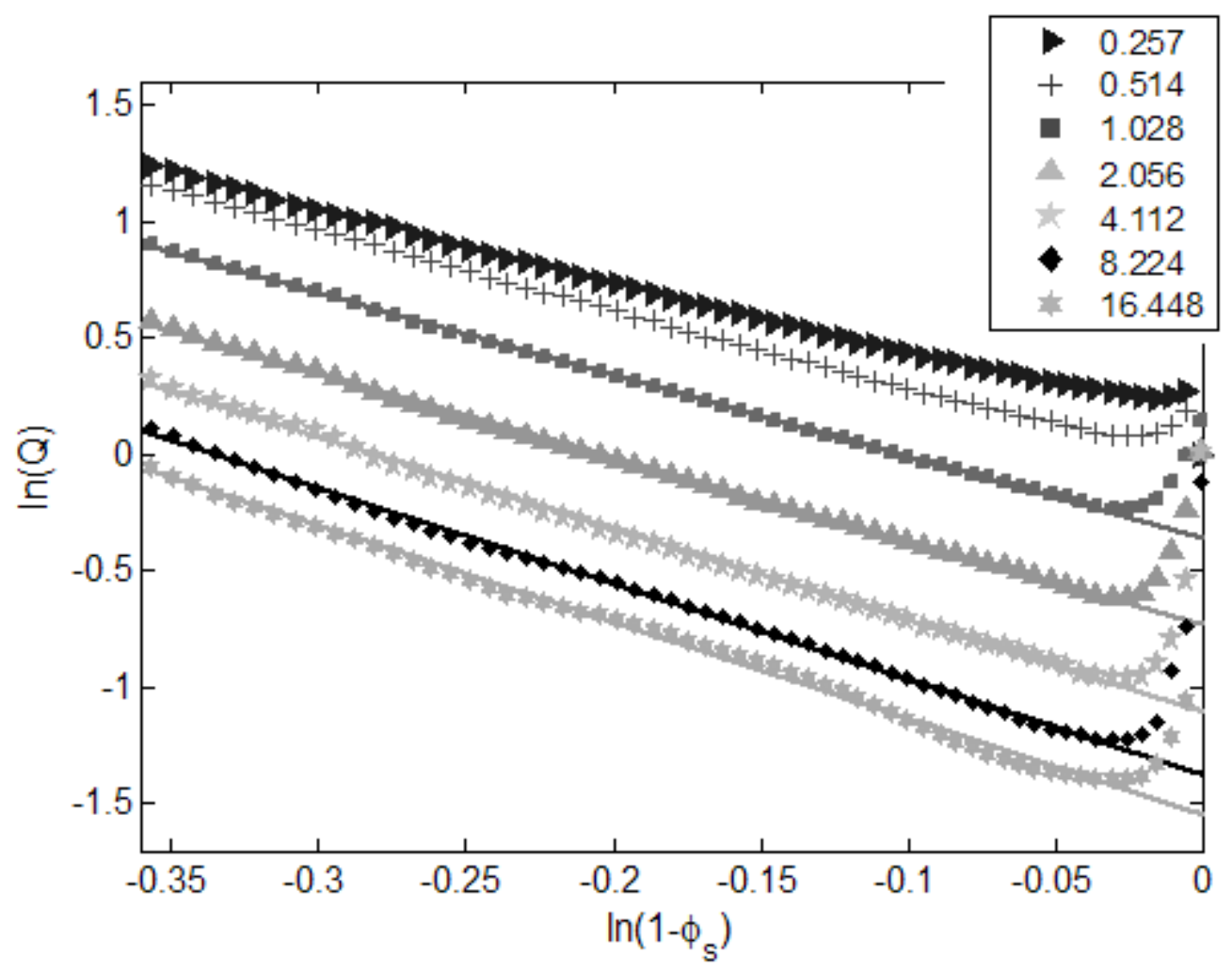

Figure 8: The results shown earlier in Figure 6a are plotted on a natural logarithmic scale. Here $Q=\frac{\beta_{d}}{\phi_{s} \phi_{g}}$ , where $\beta_{d}$ is the dimensionless filtered drag coefficient, $\phi_{s}$ is particle volume fraction, and $\phi_{g}=1-\phi_{s}$ is the gas volume fraction. The dimensionless filter lengths are shown in the legend. 


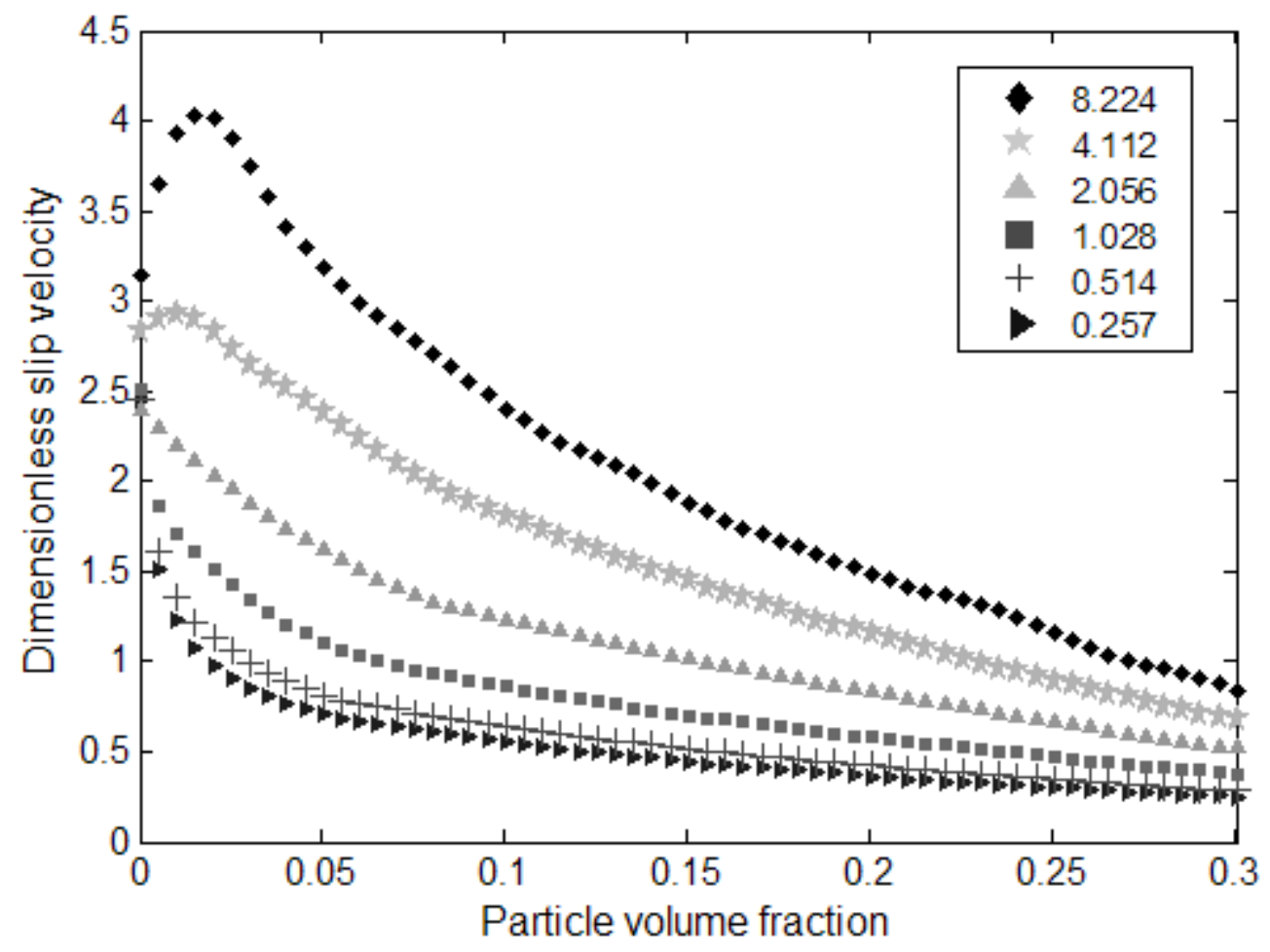

Figure 9: The variation of filtered dimensionless slip velocity with filtered particle volume fraction is shown for various dimensionless filter lengths shown in the legend. These results were generated from the same set of simulation data that led to Figure $6 \mathrm{a}$. 


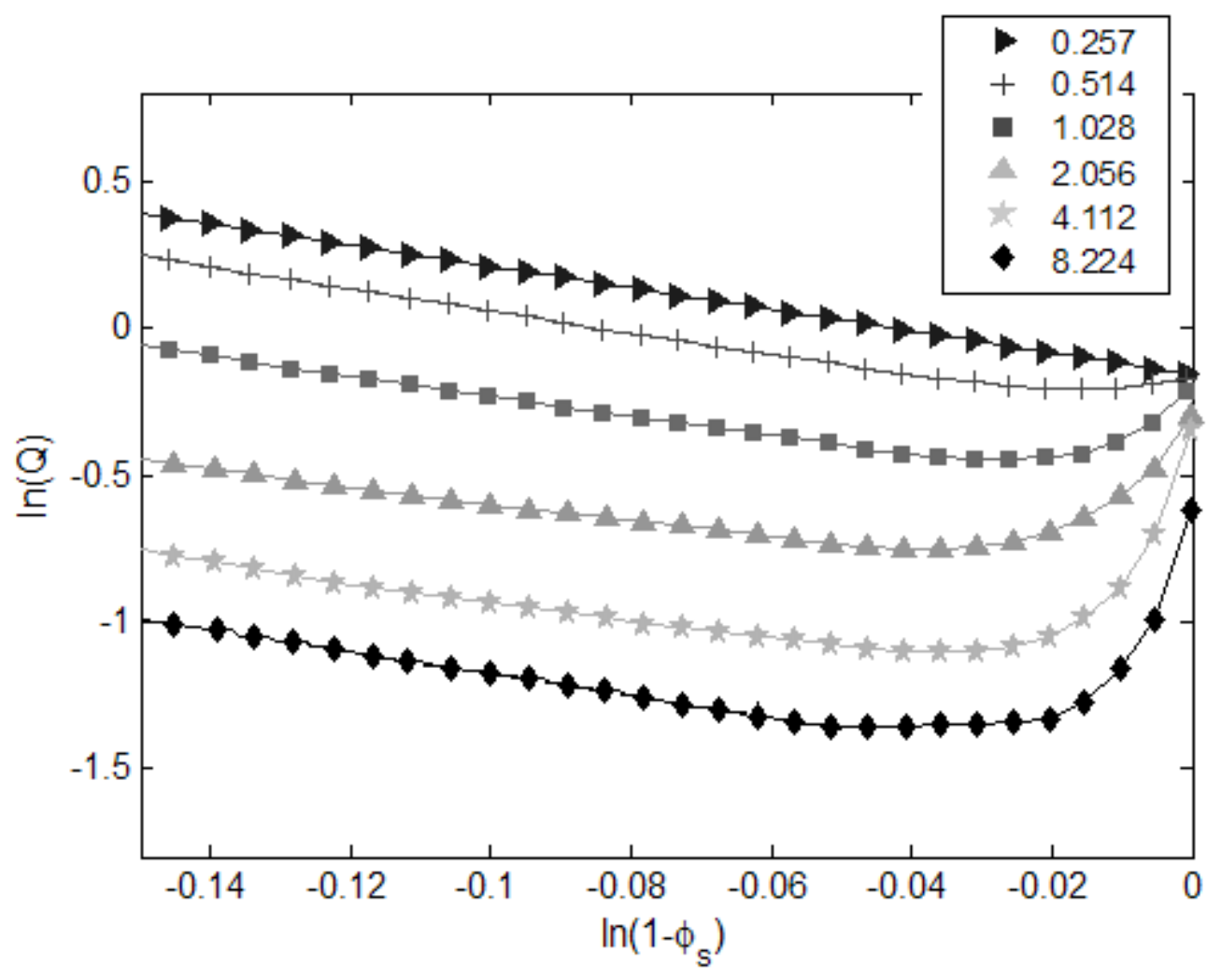

Figure 10: These results are analogous to those shown earlier in Figure 8, with the only difference being that the intrinsic drag force model used in the simulations which led to the present figure did not include a Reynolds number dependence. The dimensionless filter lengths are shown in the legend. 


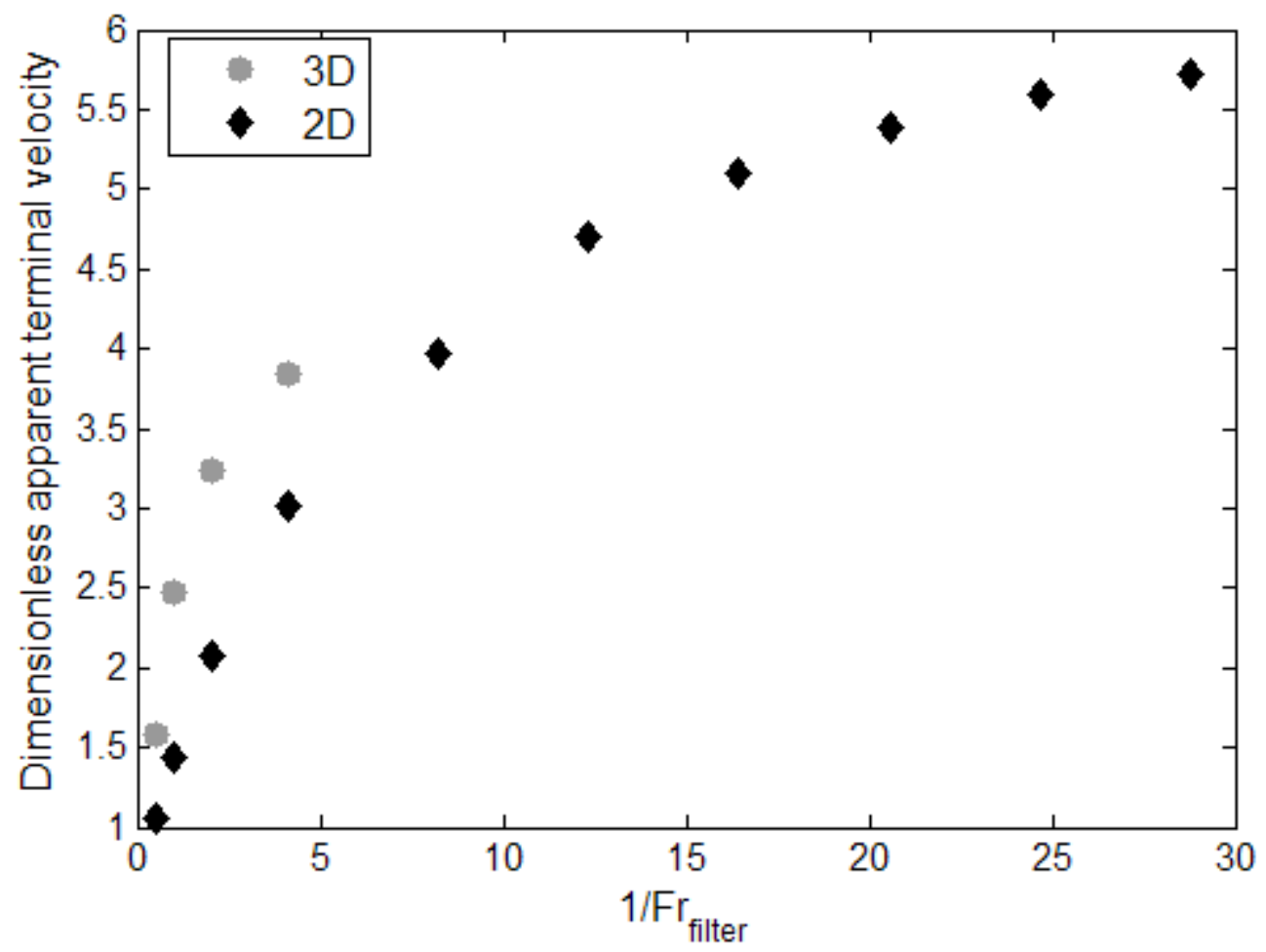

Figure 11a: Dimensionless apparent terminal velocity for different dimensionless filter lengths, extracted from results in Figure 8 (2D) for the range $0.075 \leq \phi_{s} \leq 0.30$ and those extracted from results in Figure 19 (3D) for the range $0.075 \leq \phi_{s} \leq 0.25$. The solid line in Figure 8 for a filter length of 2.056 is based on the apparent terminal velocity shown here and the apparent Richardson-Zaki exponent in Figure 11b. 


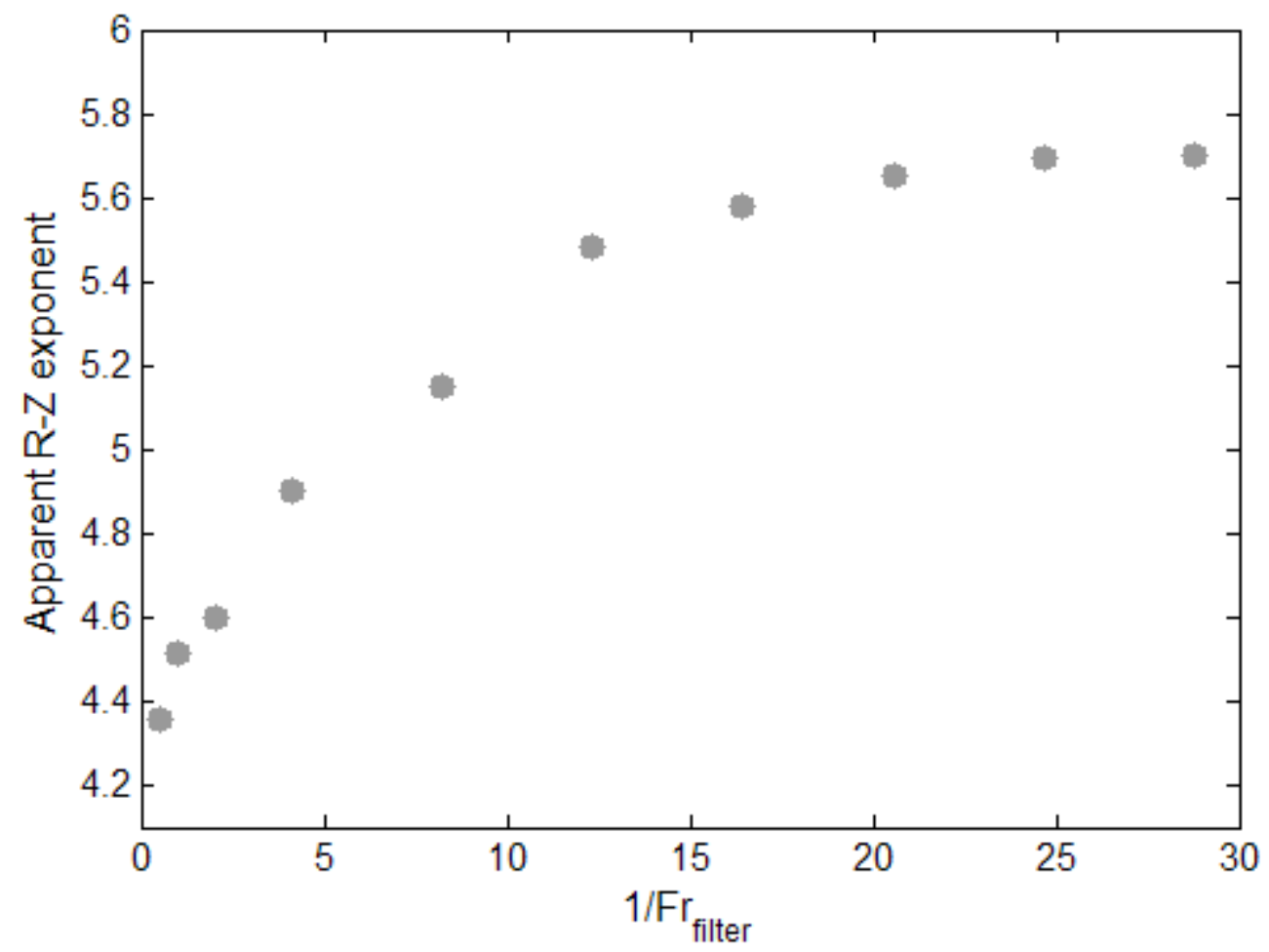

Figure 11b: Apparent Richardson-Zaki exponent for different dimensionless filter lengths, extracted from results in Figure 8 (2D) for the range $0.075 \leq \phi_{s} \leq 0.30$. The solid line in Figure 8 for a filter length of 2.056 is based on this apparent terminal velocity in Figure 11a and the apparent Richardson-Zaki exponent shown here. 


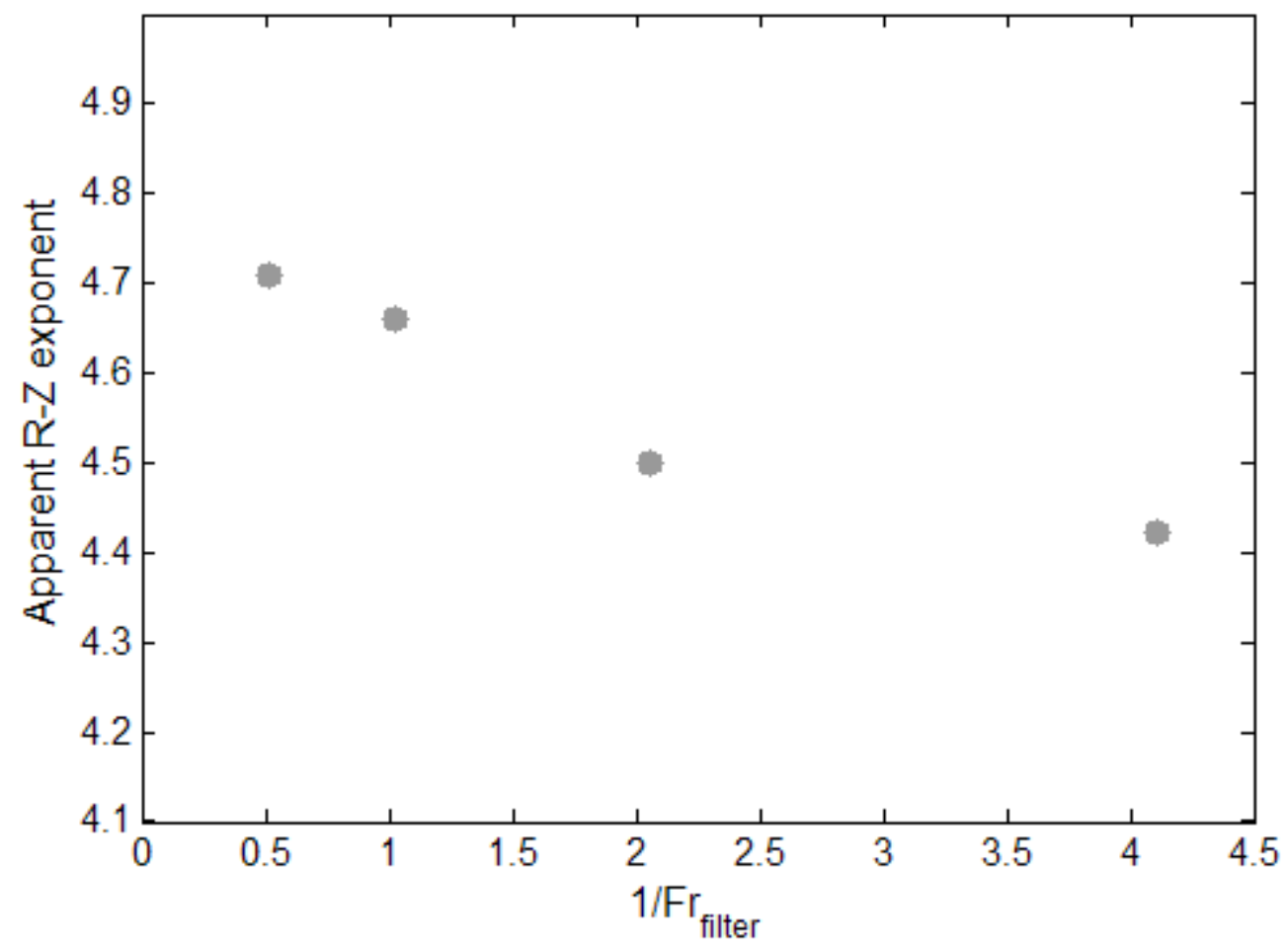

Figure 11c: Apparent Richardson-Zaki exponent for different dimensionless filter lengths, extracted from results in Figure 19 (3D) for the range $0.075 \leq \phi_{s} \leq 0.25$. 


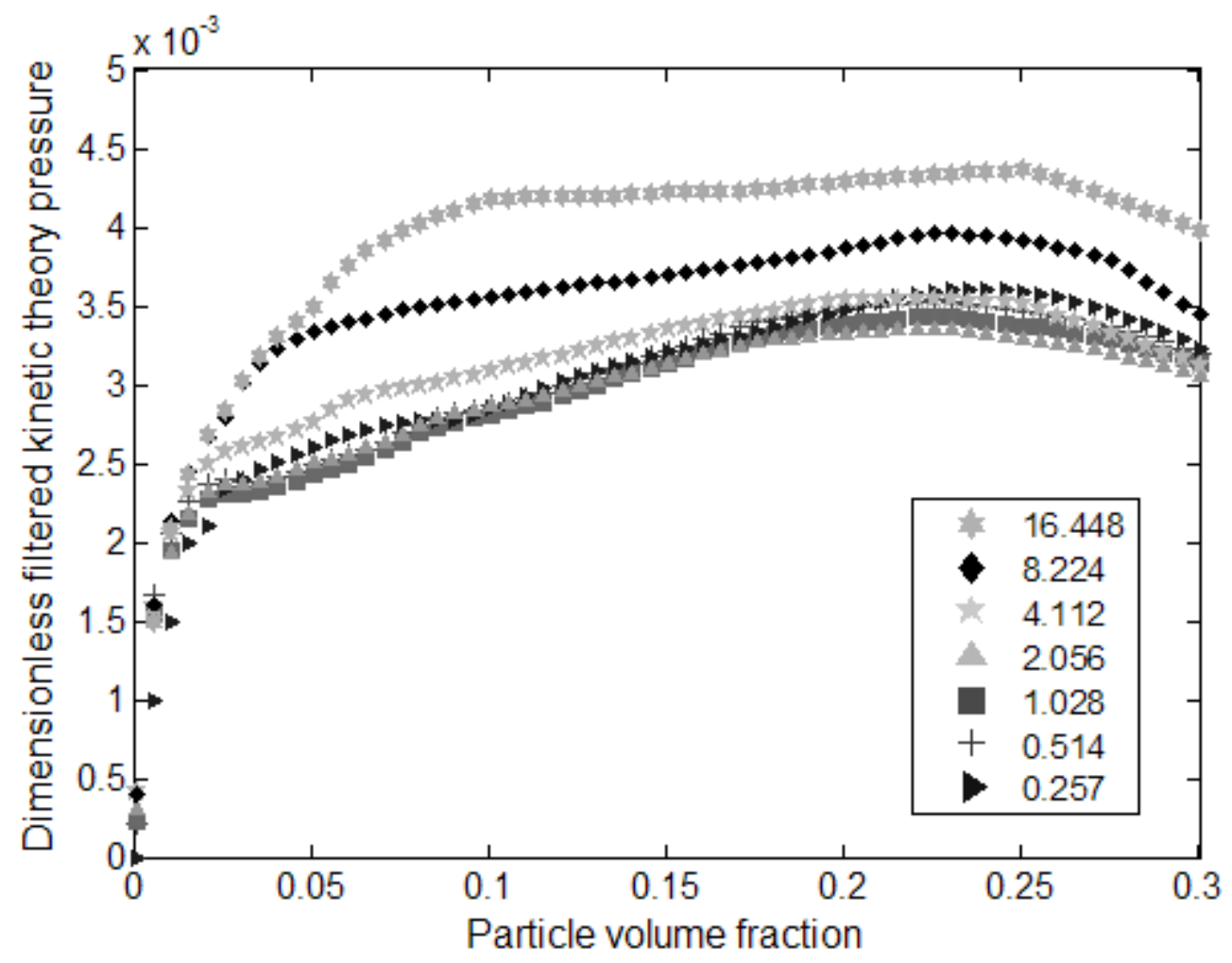

Figure 12a: The variation of the dimensionless filtered kinetic theory pressure with particle volume fraction is presented for different dimensionless filter lengths. The results were extracted from simulations mentioned in the caption for Figure 6a. The dimensionless filter lengths are shown in the legend. 


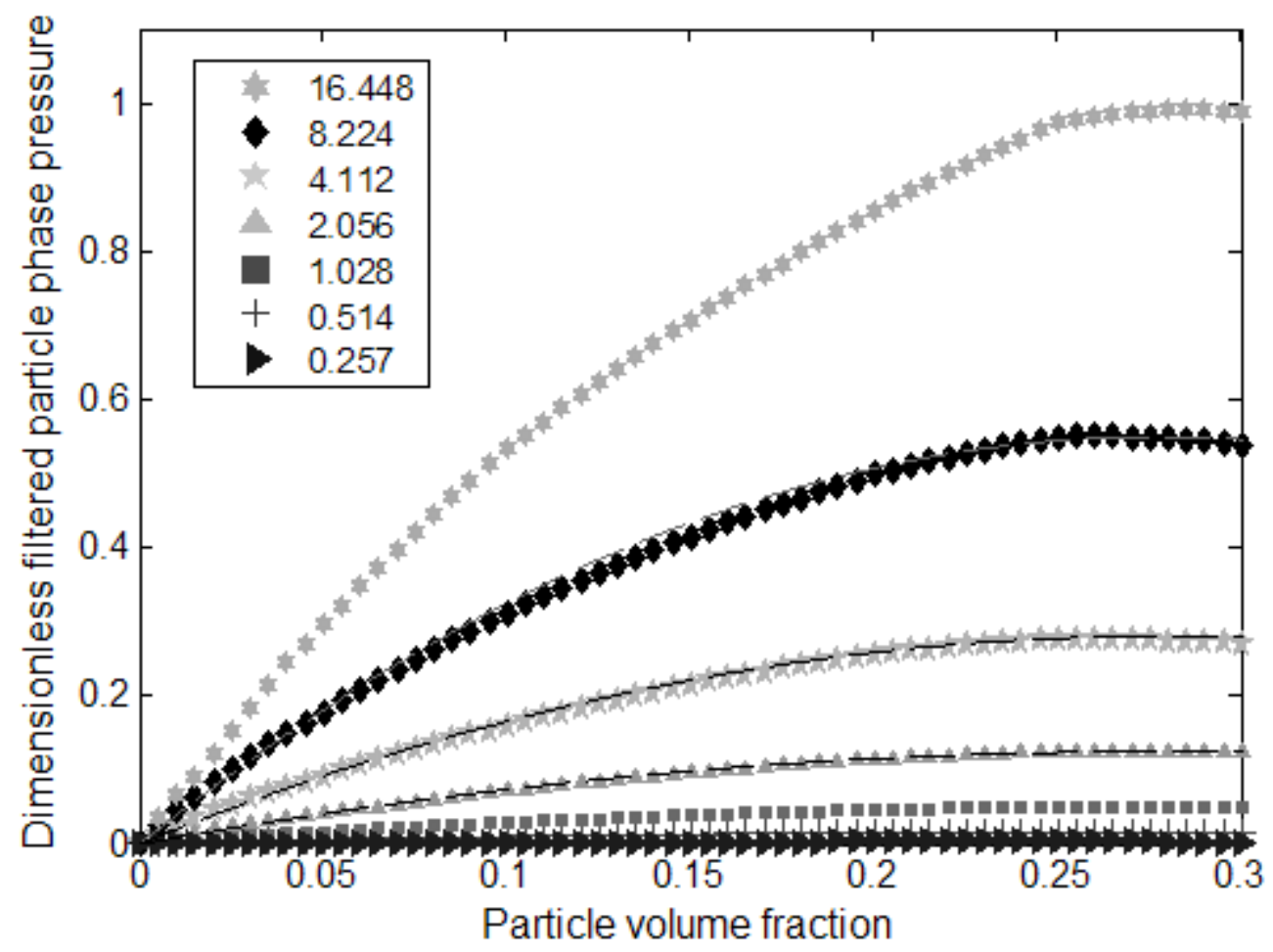

Figure 12b: The variation of the dimensionless filtered particle phase pressure with particle volume fraction is presented for different dimensionless filter lengths. The results were extracted from simulations mentioned in the caption for Figure 6a. The dimensionless filter lengths are shown in the legend. 


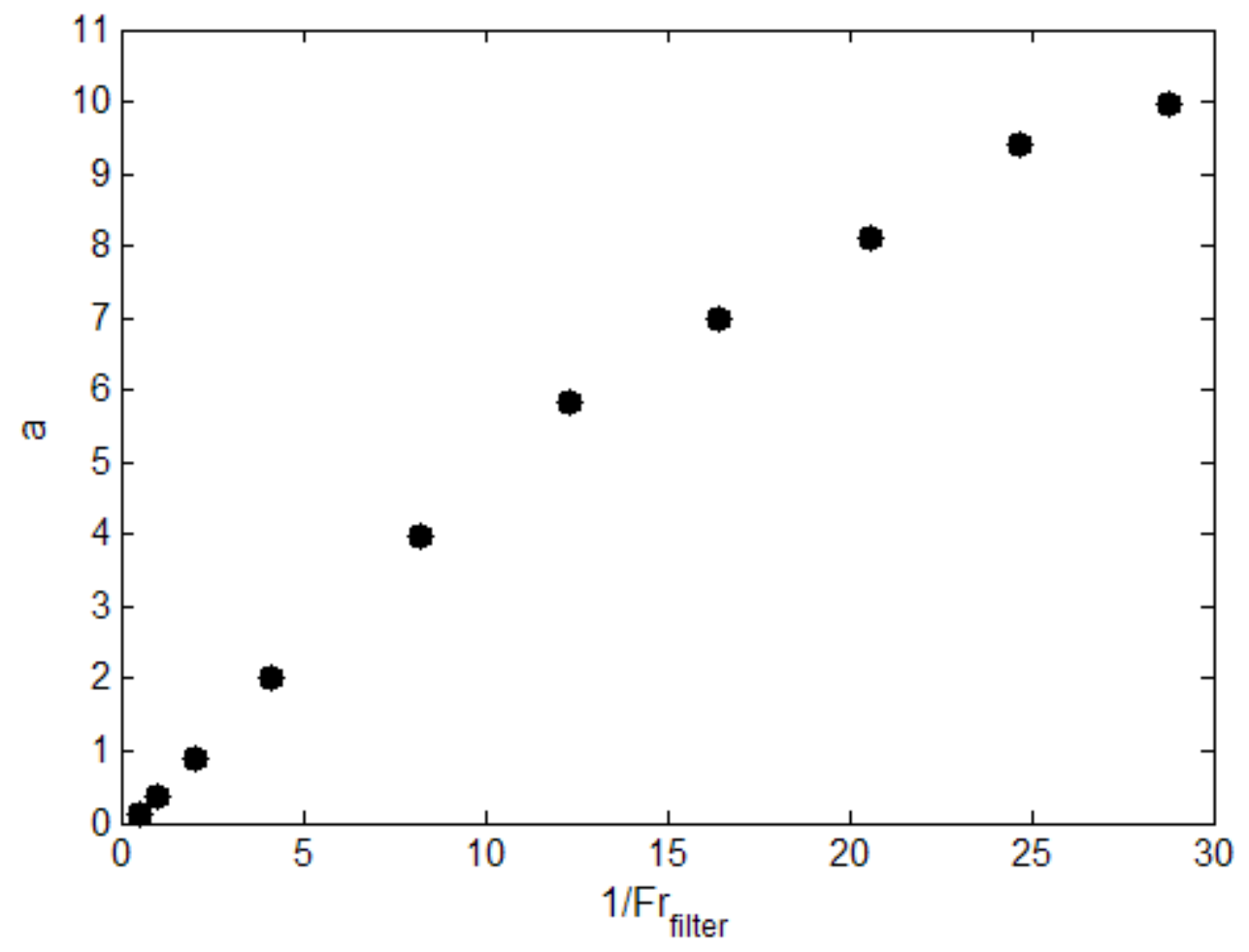

Figure 12c: The coefficient " $a$ " of the dimensionless filtered particle phase pressure in Figure 12b represented as $a \phi_{s}\left(1-b \phi_{s}\right.$ ) (for $\phi_{s} \leq 0.30$ ) is plotted against the dimensionless filter length. $b \sim 1.80$ for all filters. 


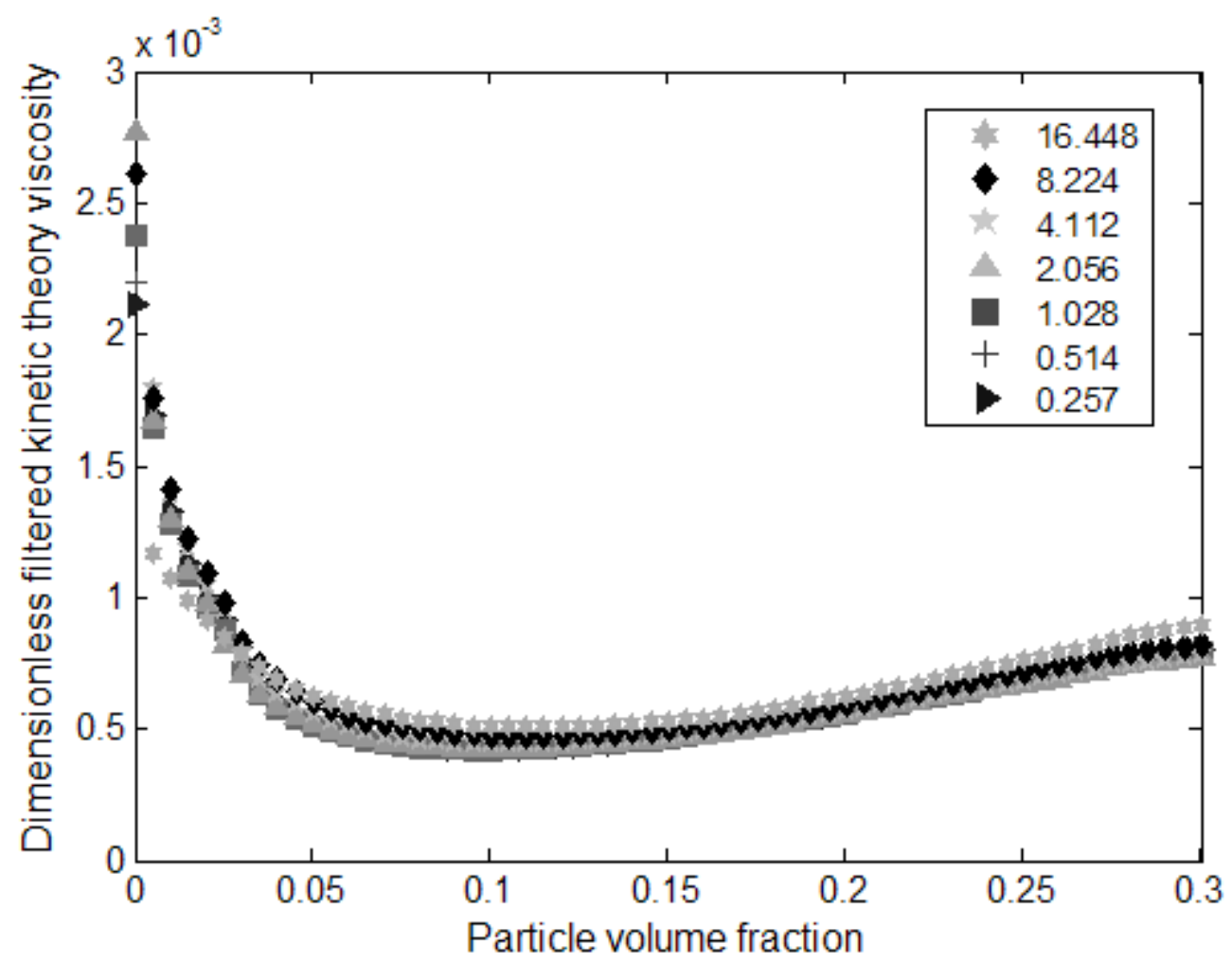

Figure 13a: The variation of the dimensionless filtered kinetic theory viscosity with particle volume fraction is presented for different dimensionless filter lengths. The results were extracted from simulations mentioned in the caption for Figure 6a. The dimensionless filter lengths from the top curve to the bottom curve are shown in the legend. 


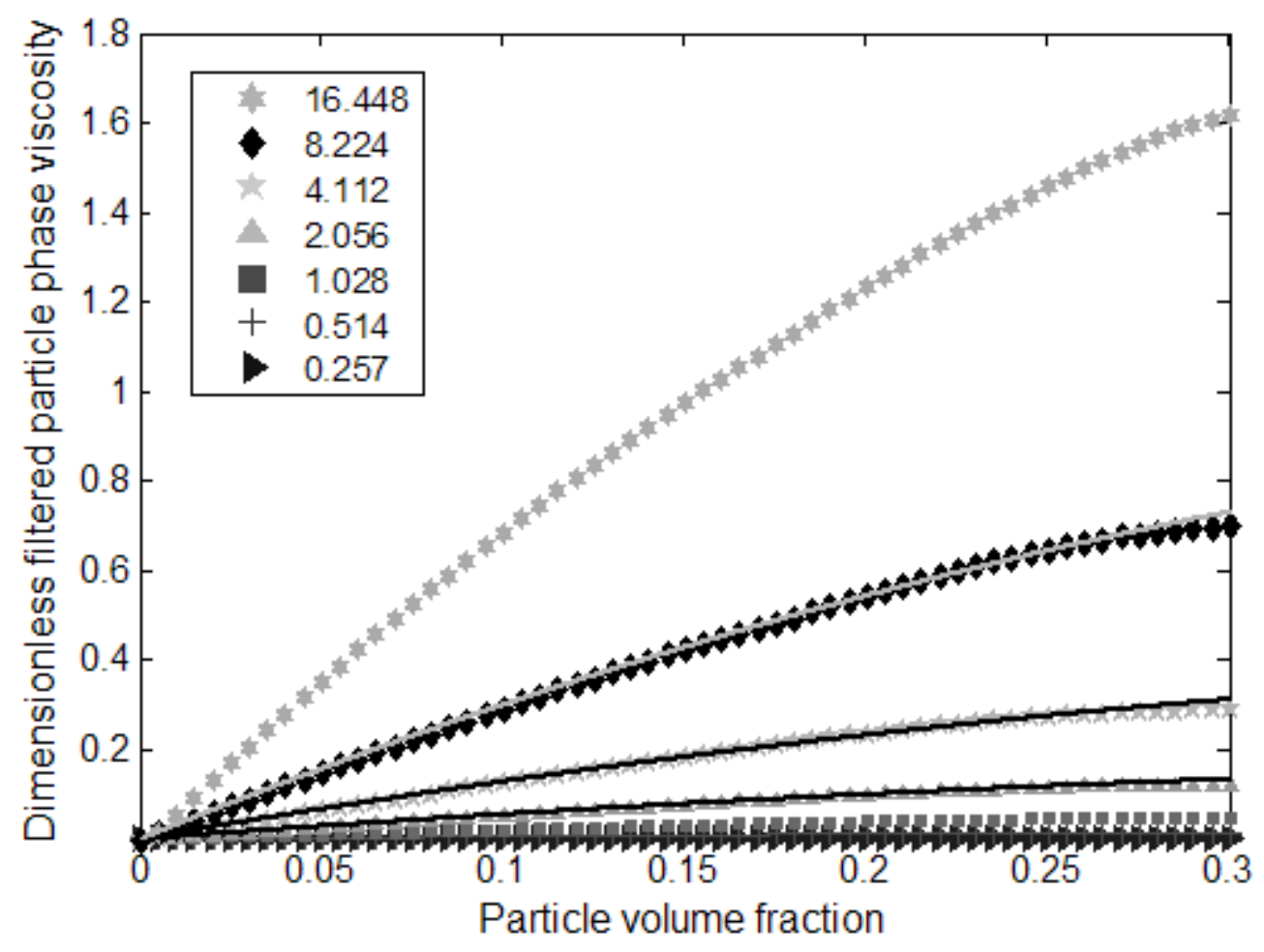

Figure 13b: The variation of the dimensionless filtered particle phase viscosity with particle volume fraction is presented for different dimensionless filter lengths. The results were extracted from simulations mentioned in the caption for Figure 6a. The dimensionless filter lengths are shown in the legend. 


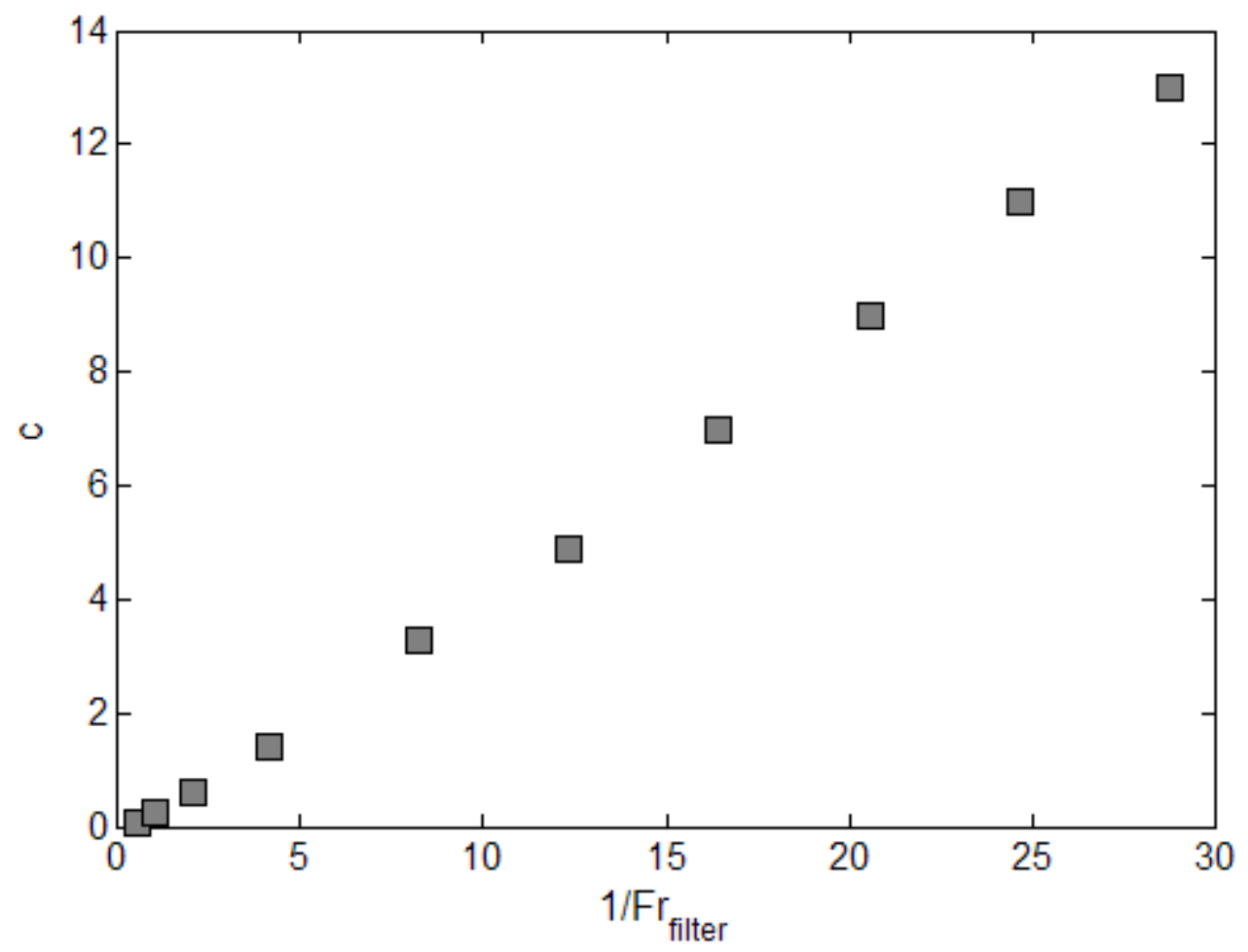

Figure 13c: The coefficient "c" of the dimensionless filtered particle phase viscosity in Figure 13b represented as $c \phi_{s}\left(1-d \phi_{s}\right.$ ) (for $\phi_{s} \leq 0.30$ ) is plotted against the dimensionless filter length. $d \sim 0.86$ for all filters. 

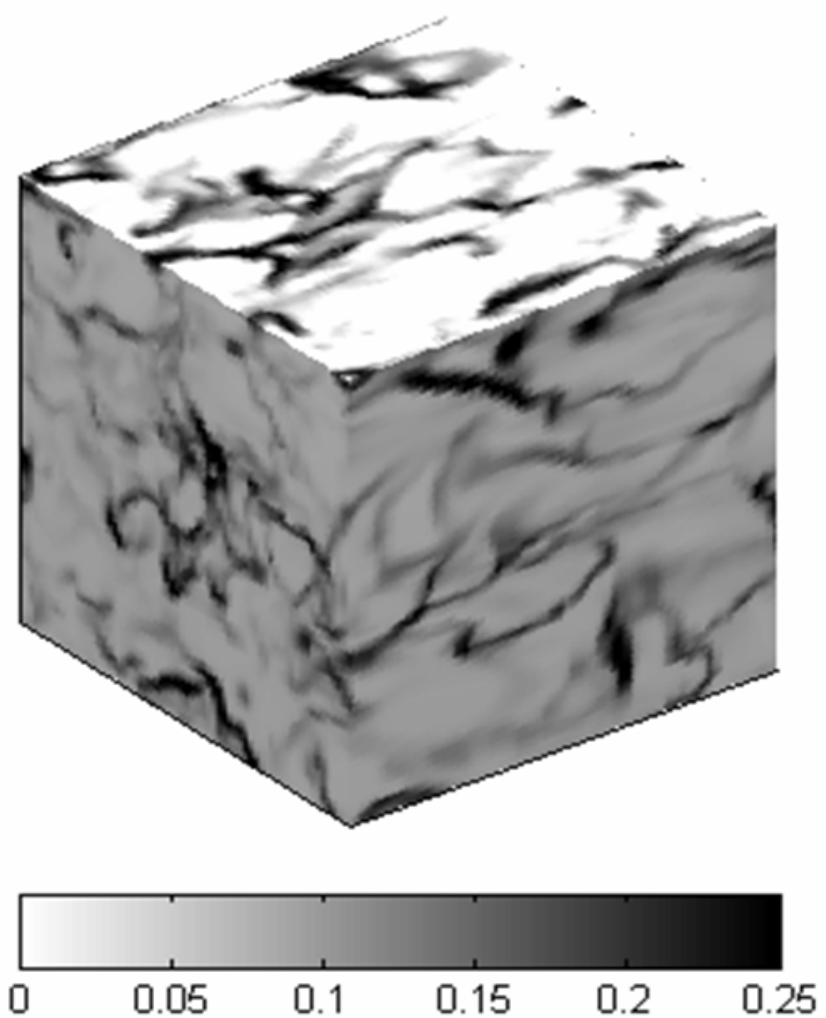

Figure 14: A snapshot of the particle volume fraction field in a large periodic domain of size $16.448 \mathrm{x}$ 16.448 x 16.448 dimensionless units is shown. Simulation was performed using 64 x 64 x 64 grid points. The domain-average particle volume fraction, $\left\langle\phi_{s}\right\rangle=0.05$. 


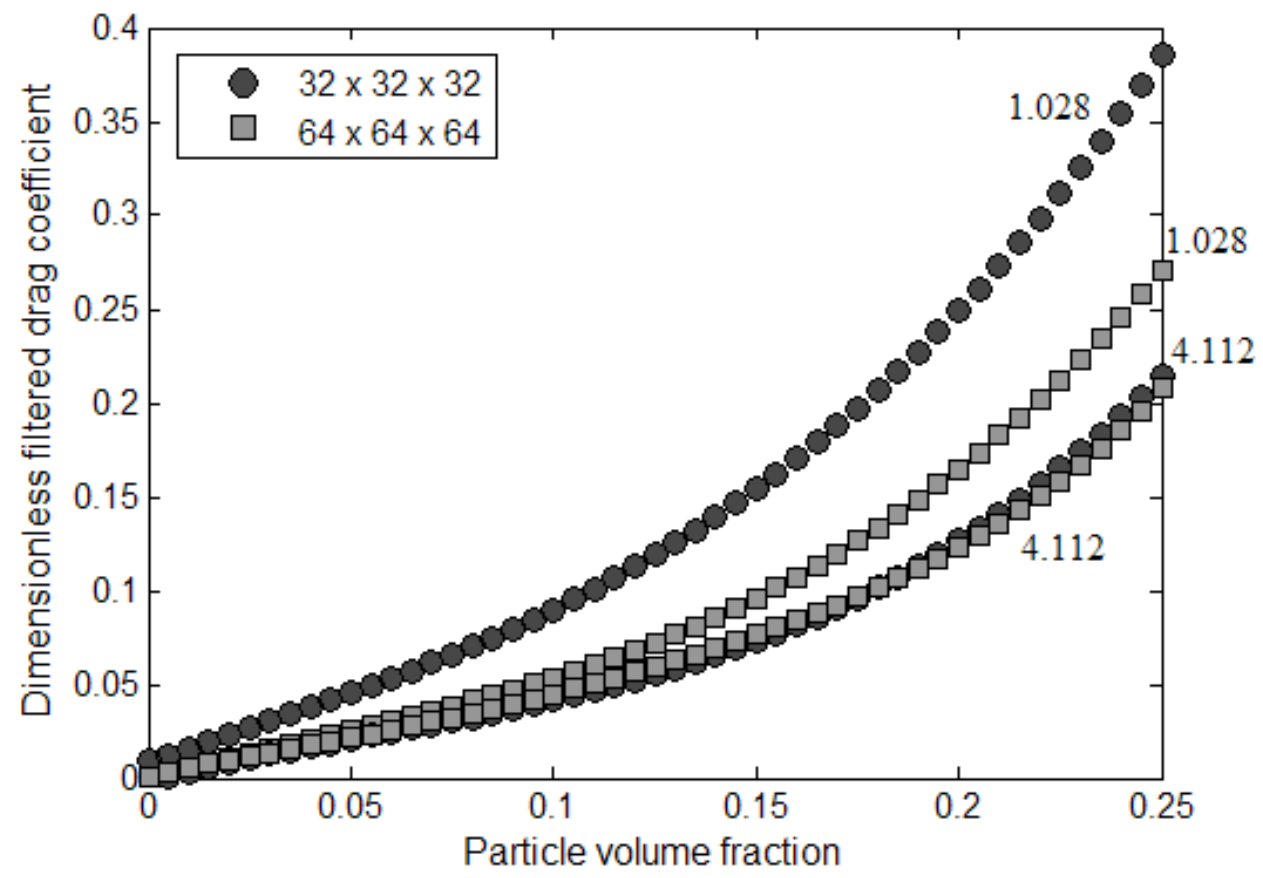

Figure 15: The effect of grid resolution on the dimensionless filtered drag coefficient is presented. Simulations were performed in a cubic periodic domain of size 16.448 x 16.448 x 16.448 dimensionless units and at two different grid resolutions. The filtered drag coefficients were calculated for dimensionless filter lengths of 1.028 and 4.112. Data used for filtering were generated by running simulations for domain-average particle volume fractions of $0.05,0.10,0.15,0.20$ and 0.35 . 


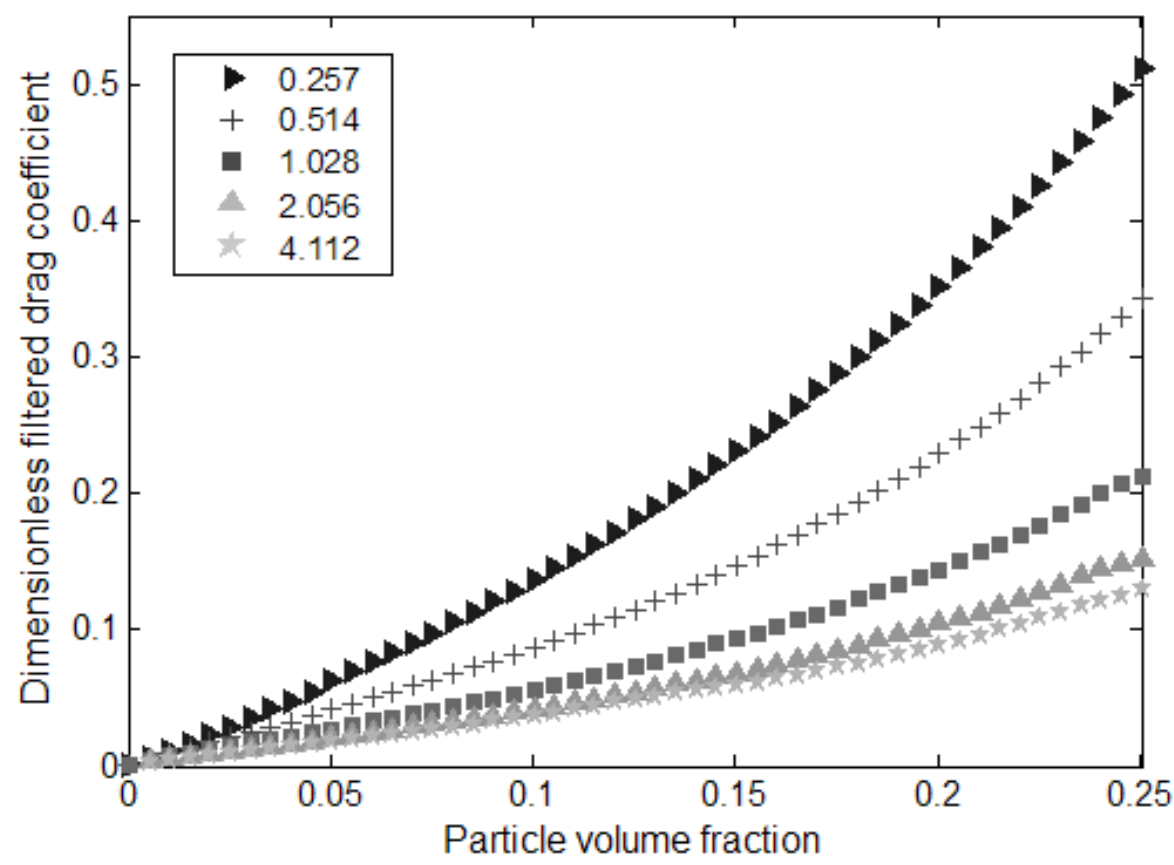

Figure 16: The variation of the dimensionless filtered drag coefficient with particle volume fraction for various filter sizes (listed in the legend in dimensionless units) is shown. Simulations were in a square domain of size 16.448 × 16.448 × 16.448 dimensionless units, using 64 x 64 x 64 grid points. Data used for filtering were generated by running simulations for domain-average particle volume fractions of 0.01 , $0.02,0.05,0.10,0.15,0.20,0.25$ and 0.35 . The dimensionless filter lengths from the top curve to the bottom curve are shown in the legend. 


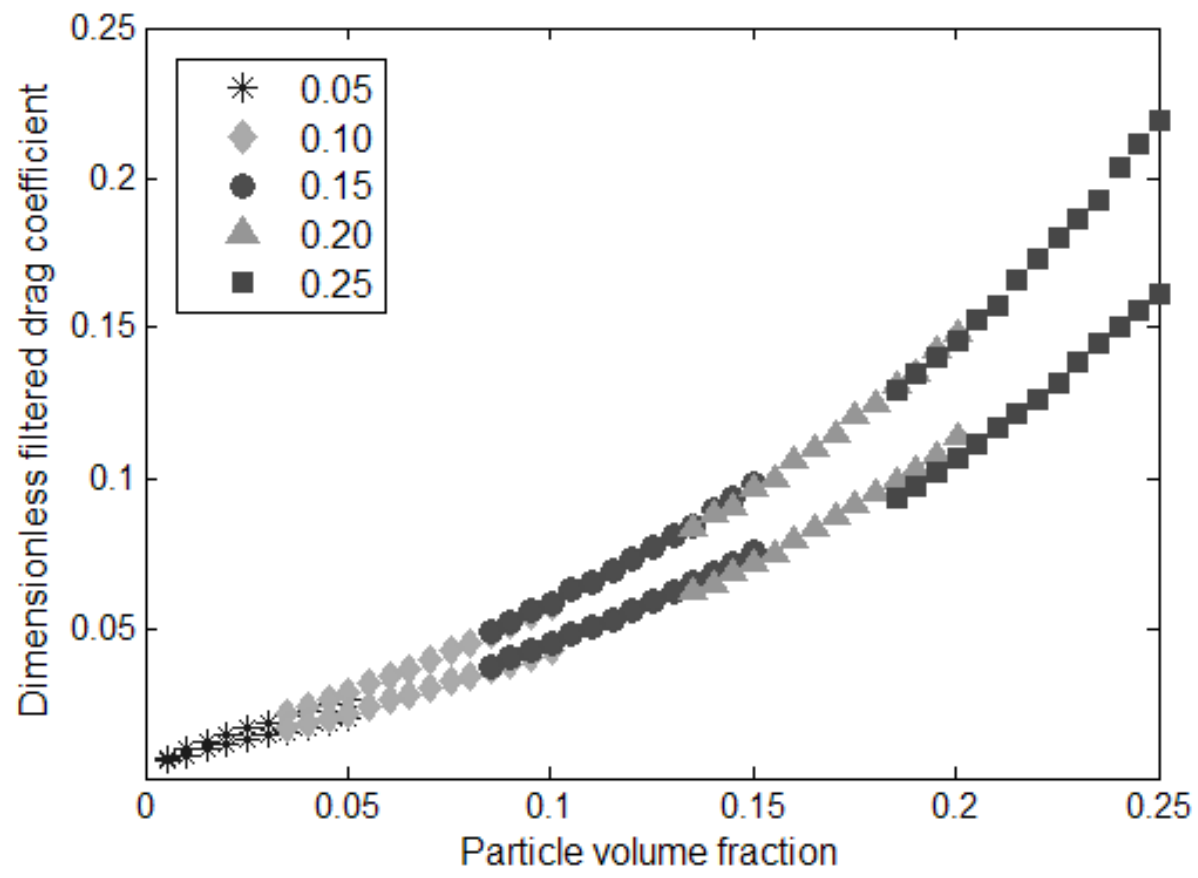

Figure 17: The effect of the domain-average particle volume fraction on the dimensionless filtered drag coefficient is presented. Simulations were performed in a cubic domain of size $16.448 \times 16.448 \times 16.448$ dimensionless units using $64 \times 64 \times 64$ grid points. The filtered drag coefficients were calculated for dimensionless filter lengths of 1.028 (top curve) and 2.056 (bottom curve). Data used for filtering were generated by running simulations for domain-average particle volume fractions of $0.05,0.10,0.15,0.20$ and 0.25 (shown by different symbols in each curve). 


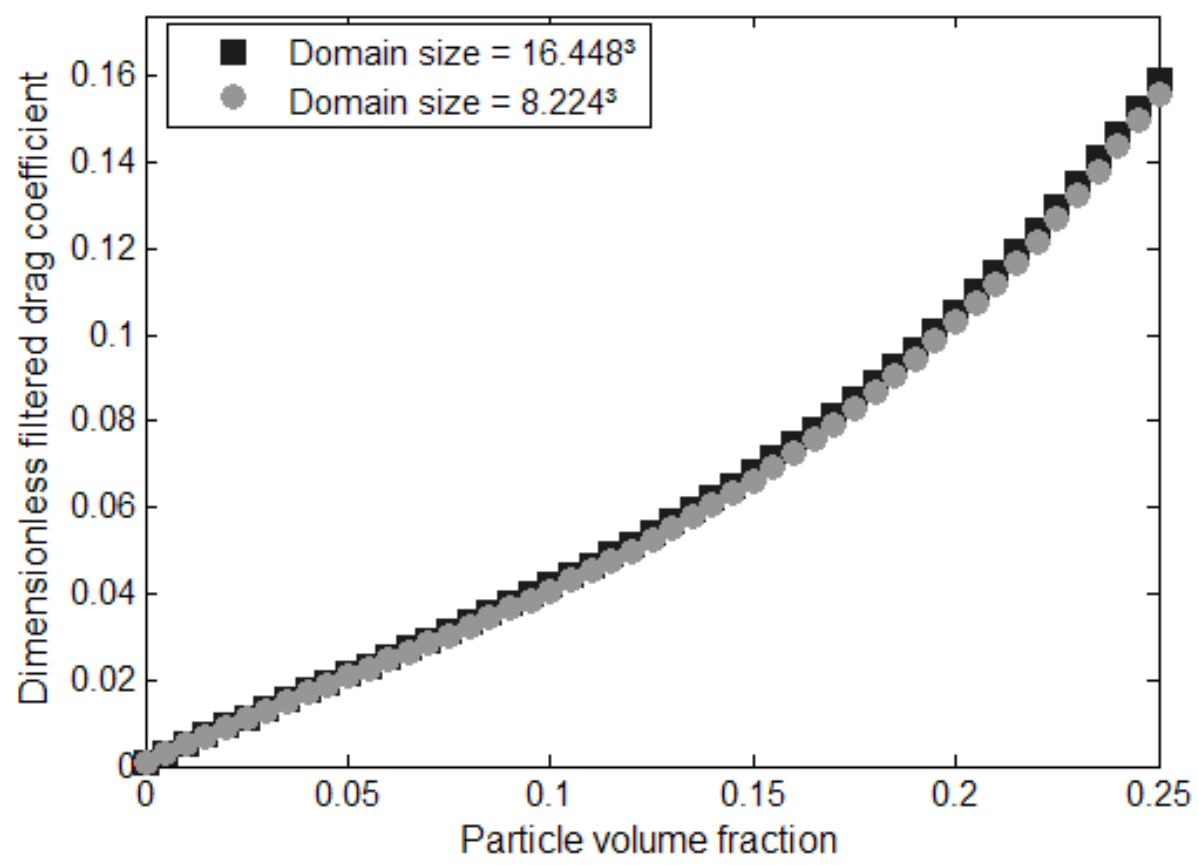

Figure 18: The effect of domain size on the dimensionless filtered drag coefficient is presented for a dimensionless filter length of 2.056. Simulations were performed at domain-average particle volume fractions of $0.05,0.15,0.25$, and 0.35 in two different cubic periodic domains of sizes: $16.448 \times 16.448 \times$ 16.448 dimensionless units ( 64 × 64 × 64 grids) and 8.224 × 8.224 × 8.224 dimensionless units $(32 \times 32 \times$ $32)$. 


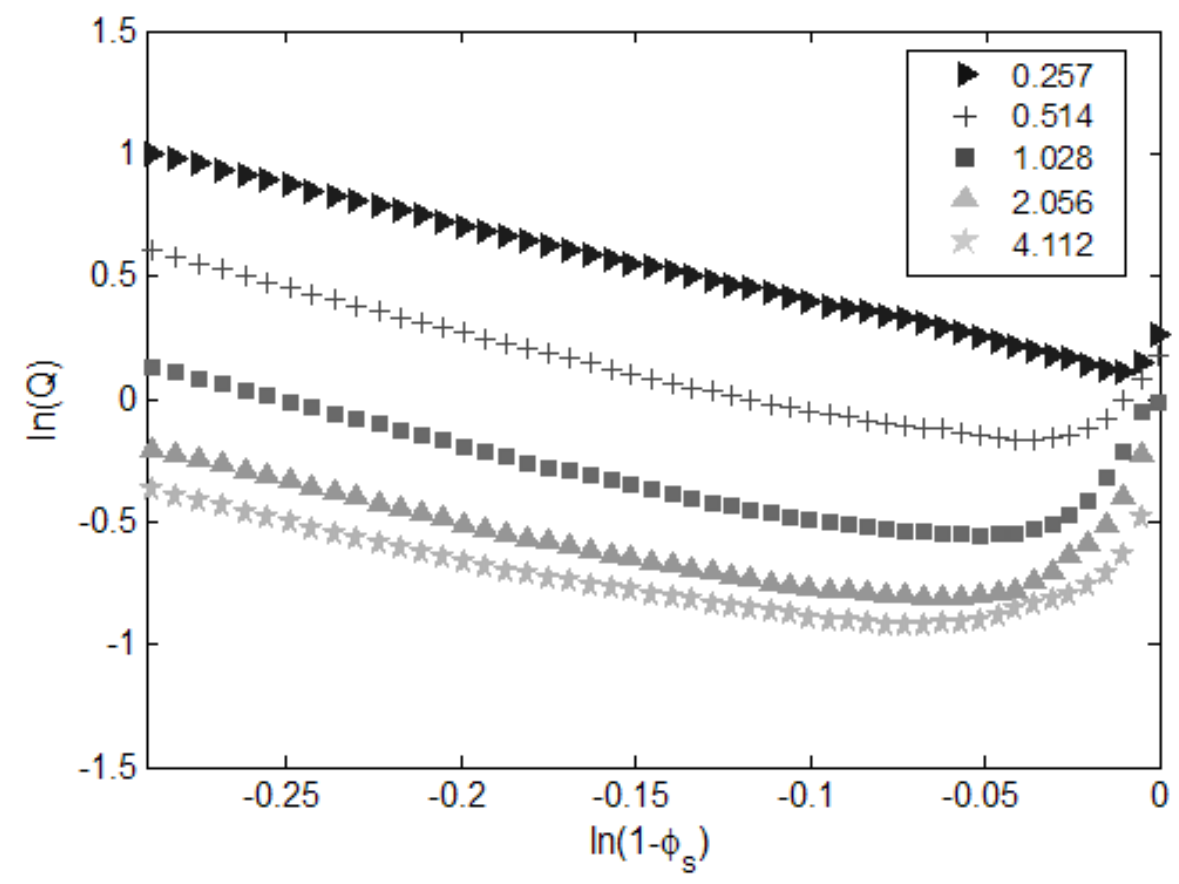

Figure 19: The results shown earlier in Figure 16 are plotted on a natural logarithmic scale. Here $Q=\frac{\beta_{d}}{\phi_{s} \phi_{g}}$, where $\beta_{d}$ is the dimensionless filtered drag coefficient, $\phi_{s}$ is particle volume fraction, and $\phi_{g}$ $=1-\phi_{s}$ is the gas volume fraction. The dimensionless filter lengths are shown in the legend. 


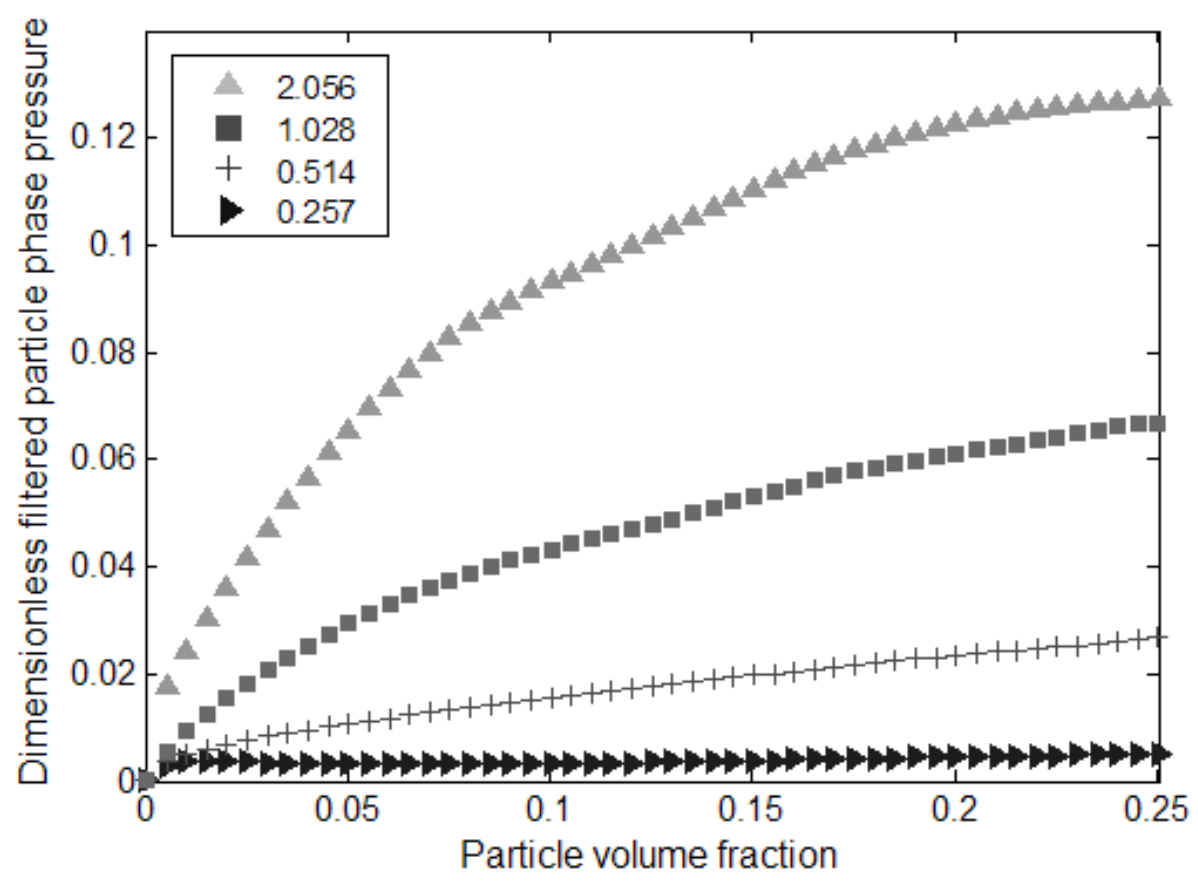

Figure 20: Dimensionless filtered particle phase pressure for different dimensionless filter lengths, extracted from simulations mentioned in the caption for Figure 16. The dimensionless filter lengths are shown in the legend. 


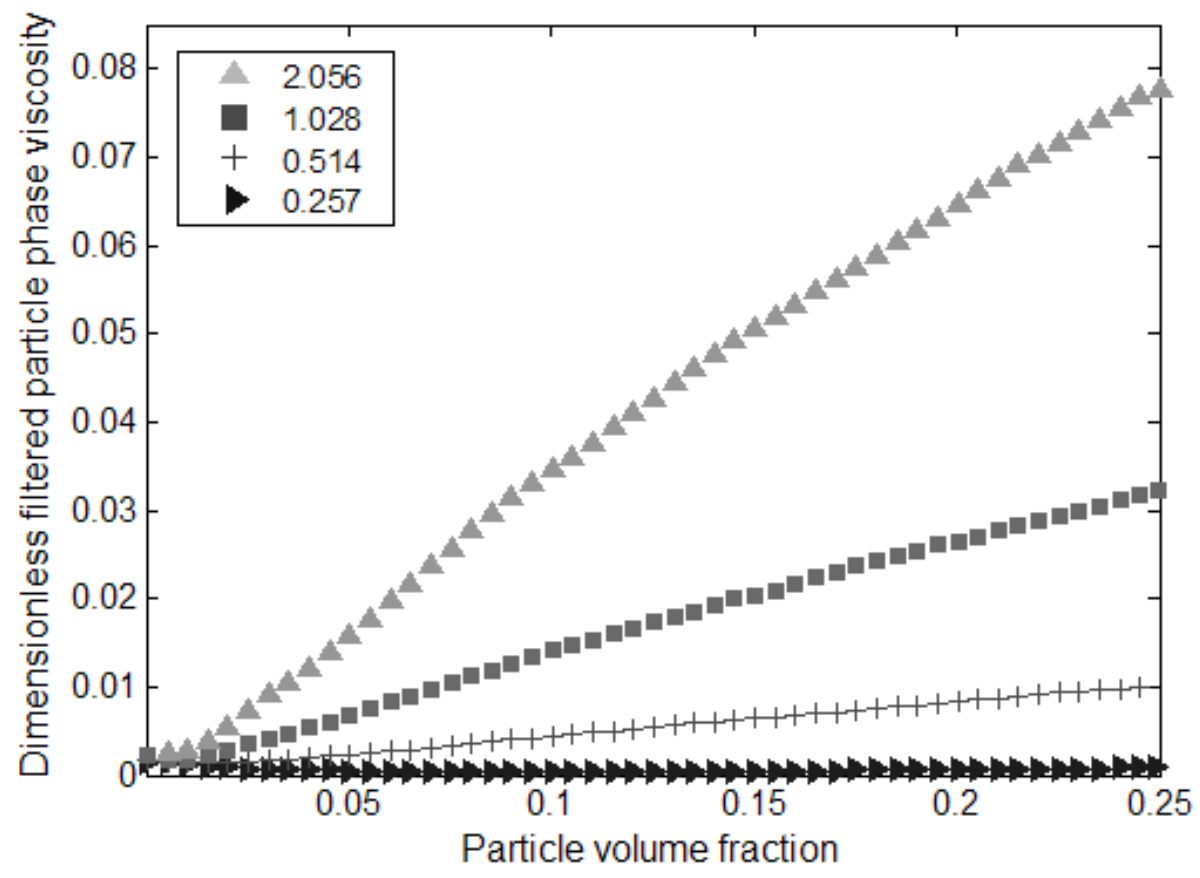

Figure 21: Dimensionless filtered particle phase viscosity for different dimensionless filter lengths, extracted from simulations mentioned in the caption for Figure 16. The dimensionless filter lengths are shown in the legend. 

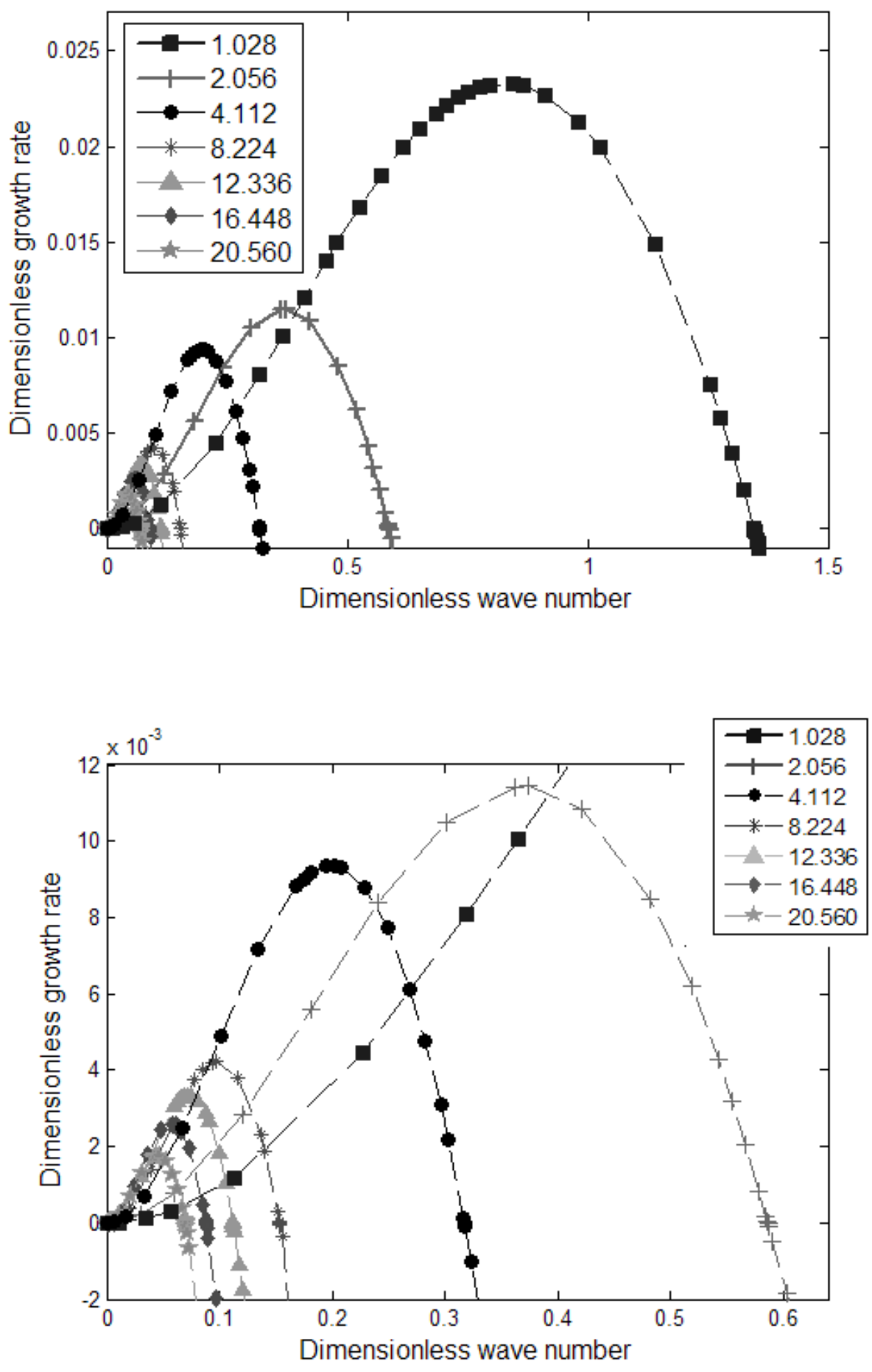

Figure 22a-b: 1D Linear stability analysis (LSA) of the filtered equations extracted from the 2D simulations for various dimensionless filter length shown in the legend. $-\left\langle\phi_{s}\right\rangle=0.15$. Part (b) is a closer snapshot of part (a). 
APPENDIX B 



\title{
Verification of filtered two-fluid models for gas-particle flows in risers
}

\author{
by \\ Yesim Igci and Sankaran Sundaresan* \\ Department of Chemical Engineering, Princeton University, Princeton, NJ 08544
}

Submitted for Publication to: AIChE Journal, June 2010

* Corresponding author: 1(609) 258-4583; sundar@princeton.edu 


\begin{abstract}
:
To investigate the effect of solid boundaries on the closure relationships for the filtered two-fluid models (applicable in the particle volume fraction range typical of riser flows), we have performed a set of the highly-resolved kinetic theory based two-fluid model simulations in 2D channels equipped with bounding walls and inlet and outlet and extracted filtered closures. The closures for the filtered drag coefficient and particle phase stress are found to depend not only on particle volume fraction and the filter length, but also on the distance from the wall. The wall corrections to the filtered closures are nearly independent of the filter length and particle volume fraction. Simulations of filtered model equations were found to yield grid length independent solutions when the grid length is $\sim$ half the filter length or smaller. It is demonstrated that the coarse statistical results obtained by solving the filtered models with different filter lengths were the same and corresponded to those from highly resolved simulations of the kinetic theory model, which was used to construct the filtered models, thus verifying the fidelity of the filtered modeling approach.
\end{abstract}




\section{Introduction}

Gas-particle flows in bubbling and circulating fluidized beds are inherently unstable, and they manifest fluctuations in velocities and local suspension density over a wide range of length and time scales. ${ }^{1,2}$ In riser flows, these fluctuations are associated with the random motion of the individual particles (typically characterized through the granular temperature) and with the chaotic motion of particle clusters, which play a major role in axial dispersions of particles, radial distribution of particles, chemical reaction rates, erosion, and heat transfer at the wall; in short, they affect the overall performance of circulating fluidized beds. ${ }^{3-9}$ Although two-fluid (Euler-Euler) models ${ }^{2,10,11}$ are able to capture these clusters in a robust manner, prohibitively expensive spatial and temporal resolutions are often needed to resolve the clusters at all length scales. ${ }^{12-14}$ Due to computing limitations, the grid sizes used in simulating industrial scale gasparticle flows are invariably much larger than the length scales of the (finer) particle clusters. Such coarse-grid simulations for industrial scale gas-particle flows will clearly not resolve the structures which exist on sub-grid length scales; however, these small-scale unresolved structures are known to affect the resolved flow characteristics. ${ }^{14-16}$

Researchers have approached this problem of treating unresolved structures through various approximate schemes. O’Brien \& Syamlal, ${ }^{17}$ Boemer et al.., ${ }^{18}$ and Heynderickx et al. ${ }^{19}$ noted the need to correct the drag coefficient to account for the consequence of clustering and proposed corrections for the very dilute limit. Some authors have used an apparent cluster size in an effective drag coefficient closure as a tuning parameter; ${ }^{20}$ others have deduced corrections to the drag coefficient using an Energy Minimization Multi-Scale approach (EMMS). ${ }^{21-23}$ Some researchers have modified the drag coefficients for homogeneous systems using a bubbleemulsion model, where the bubble and emulsion phases are described as two interpenetrating 
phases (to capture bed-expansion characteristics in bubbling fluidized beds with coarse grid simulations). ${ }^{24,25}$ The concept of particle phase turbulence has also been explored to introduce the effect of the fluctuations associated with clusters and streamers on the particle phase stresses. $^{26,27}$

Agrawal et al. ${ }^{12}$ performed highly resolved simulations of kinetic-theory based two-fluid model (henceforth referred to as microscopic two fluid model) equations for gas-particles flow in periodic domains, determined the domain-averaged effective drag and effective stresses, and demonstrated that these effective quantities were not only quantitatively very different from those used in the microscopic two-fluid model, but also depended on size of the periodic domain. They also found that both $2 \mathrm{D}$ and $3 \mathrm{D}$ simulations revealed the same qualitative trends. Andrews et al. ${ }^{28}$ performed highly resolved simulations of fluidized gas-particle mixtures in a $2 \mathrm{D}$ periodic domain whose total size coincided with that of the grid size in an anticipated large-scale riser flow simulation and constructed ad hoc sub-grid models for the effects of the fine-scale flow structures on the drag force and the stresses, and examined the consequence of these sub-grid models on the outcome of the coarse-grid simulations of gas-particle flow in a large-scale vertical riser. They found that these sub-grid scale corrections affect the predicted large-scale flow patterns profoundly. ${ }^{28}$ In our earlier work ${ }^{29}$, we presented a systematic filtering approach to construct closure relationships for the drag coefficient and the effective stresses in the gas and particle phases. Briefly, we performed highly resolved simulations of a kinetic theory based two-fluid model with Wen \& Yu drag for uniformly sized particles ${ }^{2,12,28-30}$ in a large periodic domain (considerably larger than the filter length) and filtered the results using different filter lengths. We showed that the closure relationships for the drag coefficient and the effective stresses in the gas and particle phases (that appear in the filtered two-fluid model) manifested a 
definite and systematic dependence on the filter length. However, these filtered closures did not include the possible effect of bounding walls and are therefore likely to be restricted to flow regions far away from solid boundaries.

An alternate approach based on the aforementioned EMMS method has been developed by $\mathrm{Li}$, Kwauk, and coworkers. ${ }^{31-35}$ Such an approach is reported to have good success in capturing experimental data. ${ }^{22,36}$ Unlike the present study where the corrections to the drag force depend on filter size, the EMMS model prescribes a fixed modification to the drag force and it may perhaps be viewed as the large filter length limit. Some authors ${ }^{13,37-42}$ have combined EMMS model (which assumes that some of the particles reside in a clustered state) for drag with kinetic theory model for stresses (which assumes that individual particles move chaotically); in contrast, the approach pursued in our studies mentioned above and in the present study filters the stresses and the drag in a consistent manner.

It is now generally accepted that clusters and streamers are formed in gas-particle flows in vertical risers and they are found more frequently near the tube walls so that on an average, particle volume fraction is larger near the wall region. A consequence of such segregation is that the average velocity of particles and gas in the wall region can be downward even though the net flow is in the upward direction - a dilute rising core and a dense descending annular region., 5,9 , 15, 43, 44 Clusters at the wall of a riser have been observed to form, descend, break-up, travel laterally from the annulus to the core and then be re-entrained in the upward flowing core. In this manner, they contribute to the internal solids mixing process within a riser. ${ }^{7,8}$ Consequently, it is important to incorporate the effects of bounding walls on the filtered closures before attempting a comparison of the filtered model predictions with experimental data. 
The first objective of the present study is to investigate of the effect of the bounding walls on the closure relationships for the filtered two-fluid model equations and to incorporate these effects as wall corrections to the filtered drag coefficient and particle phase stresses that are appropriate for coarse-grid simulations of gas-particle flows in risers (of circulating fluidized beds). To address this point, we have performed a set of highly resolved simulations of a kinetic-theory based two-fluid model in 2D channels equipped with bounding walls and inlet and outlet regions, and analyzed the variation of the filtered closures with distance from the bounding walls using different filter lengths. The present analysis reveals that the filtered quantities must be allowed to depend not only on particle volume fraction, but also on the distance from the wall.

The second objective of the present study is to demonstrate the fidelity of the filtered twofluid models. This entails several tests: (a) the filtered two-fluid models must yield grid-length independent coarse statistical quantities once the grid length has become sufficiently smaller than the filter length; (b) these coarse statistical quantities obtained with different filter lengths should be the same, at least for a range of filter lengths; and (c) the coarse statistical quantities obtained by solving the filtered model must match those obtained by through highly resolved solution of the microscopic two-fluid model (which was used to generate the filtered two-fluid model). It will be demonstrated in this study that all of these requirements are indeed met satisfactorily, thus establishing the viability of this approach. Finally, we will also present some results on the CPU requirements for computations using the filtered model and contrast it with those for highly resolved simulations of the microscopic two-fluid model. 


\section{The effect of bounding walls on closures for filtered two-fluid model equations}

To probe the effect of solid boundaries on the closure relations, we simulated the flow of a mixture of uniformly sized particles and gas through vertical 2D channels equipped with bounding walls and inlet and outlet regions (see Figure 1). The kinetic theory based two-fluid model equations and the associated constitutive relations used in these simulations can be found in Agrawal et al. ${ }^{12}$ Partial-slip boundary conditions developed by Johnson and Jackson ${ }^{45}$ were used for the tangential velocities and granular temperature $\left(\Theta_{s}\right)$ of the uniformly-sized particle phase at all walls. These boundary conditions are as follows:

$$
\begin{aligned}
& \underline{\mathbf{n} \cdot \sigma_{s}} \cdot \mathbf{t}+\frac{\pi}{2 \sqrt{3} \phi_{s, \max }} \varphi \rho_{s} g_{o} \phi_{s} \sqrt{\Theta_{s}} \mathbf{v}_{\mathrm{sl}}=0, \quad \mathbf{v}_{\mathrm{sl}}=\mathbf{v}-\mathbf{v}_{\mathrm{w}} \\
& \mathbf{n} \cdot \mathbf{q}=\frac{\sqrt{3} \pi}{6 \phi_{s, \max }} \varphi \rho_{s} \phi_{s} g_{o} \Theta_{s}^{1 / 2}\left|\mathbf{v}_{\mathrm{sl}}\right|^{2}-\frac{\sqrt{3} \pi}{4 \phi_{s, \max }}\left(1-e_{\mathrm{w}}^{2}\right) \rho_{s} \phi_{s} g_{o} \Theta_{s}^{3 / 2}, \quad \mathbf{q}=-\lambda_{s} \nabla \Theta_{s}
\end{aligned}
$$

Here, $\varphi$ represents the specularity coefficient, which is a measure of the fraction of collisions transferring tangential momentum to the wall and varies between zero (for smooth walls) and unity (for rough walls). ${ }^{46,47}$ (Values ranging between 0.0001 and 0.6 have been reported to capture the macroscopic flow patterns in the literature ${ }^{48-51}$.) In this study, we have performed simulations for a number of different specularity coefficients between zero and one. For the gas phase, free-slip boundary condition was used at all walls. (It has already been shown that the gas/particle flow patterns in a riser are only weakly dependent on the gas phase boundary conditions. $^{48}$ ) All the simulations were done using the open-domain software MFIX. ${ }^{11}$

Although all the results will be presented as dimensionless variables, with $\rho_{s}, \mathrm{v}_{\mathrm{t}}$, and $g$ as the characteristic density, velocity, and acceleration, it is instructive to consider a typical set of 
dimensional quantities to help visualize a representative physical system better. (See Table 1 and the nomenclature for the non-dimensionalization.) Most of the $2 \mathrm{D}$ filtered results with wall corrections presented in this manuscript are based on computational data gathered in a $2 \mathrm{D}$ channel with width and height of 102.8 and 1028 dimensionless units, respectively. This domain size corresponds to $0.5 \mathrm{~m} \times 5.0 \mathrm{~m}$ for the $7.5 \times 10^{-5} \mathrm{~m}$ FCC particles and ambient air (whose properties are given in Table 1). (Henceforth "dimensionless units" will be referred to as "du".) In our simulations, the gas and particles enter the channel uniformly at the bottom. The dimensionless (dimensional) inlet gas and particle phase superficial velocities are 4.259 (0.93 $\mathrm{m} / \mathrm{s})$ and $0.109(0.0238 \mathrm{~m} / \mathrm{s})$, respectively. The inlet particle phase volume fraction is 0.07 . A splash plate is located over the entire width at the top. The gas and particles are allowed to leave through exit regions located on either side (just below the splash plate). The height of this opening is one-half the width of the system. This geometry is similar to the one employed in Andrews et $a .^{28}$, although the dimensions of the channel are now smaller to make highly resolved simulations affordable.

In each simulation, after an initial transient period that depended on the initial conditions, persistent, time-dependent and spatially inhomogeneous structures developed (henceforth referred to as statistical steady state, SSS). Figures $2 \mathrm{a}$ and $2 \mathrm{~b}$ show instantaneous snapshots of the particle phase volume fraction field in the SSS for simulations with wall specularity coefficients of 0.0001 and 0.6, respectively. By analyzing thousands of such snapshots, the closure relations for the filtered models were obtained with various filter lengths. The procedure to extract the filtered closure relations is similar to that used in our earlier study of flows in periodic domains, but also has an important difference. When simulations are done in a periodic domain, all the cells are statistically equivalent, and therefore no distinction needs to be made 
about the location; the same is not true in a riser flow simulation. Therefore, the filtered results extracted by post-processing a riser flow simulation were classified in terms of the distance from the boundaries as well as in terms of the average particle phase volume fraction inside the filtering region. Further discussion of the procedure to extract filtered closure relations can be found in our earlier study, ${ }^{29}$ Agrawal et al. ${ }^{12}$ and Andrews et al. ${ }^{28}$

In the present study, these kinetic-theory based two-fluid model simulations were performed with a grid length of $0.514 d u$ as anything finer was beyond our resources. Therefore, we used the data obtained at this resolution to do the filtering and all the filtered closure relations presented here are based on this grid resolution; there are minor quantitative, but not qualitative, differences between the closures obtained with $0.514 d u$ grids and that presented in our earlier manuscript ${ }^{29}$ for $0.257 d u$, but this difference is insignificant when the filter length is considerably larger than the grid length.

Figures $3 \mathrm{a}$ to $3 \mathrm{c}$ show the effect of the bounding walls on the filtered drag coefficient, particle phase horizontal normal stress and the particle phase shear viscosity, respectively, for 4 different particle volume fractions inside the filtering region and a filter length of $2.056 \mathrm{du}$. (We examine the horizontal normal stress as it is the most relevant normal stress component for the development of lateral segregation of particles in riser flows. The horizontal normal stress includes both the kinetic theory contribution and that arising from the mesoscale fluctuations.) All the quantities in these figures have been scaled with the corresponding values extracted from the core region (plateau values) of the channel. The filtered drag coefficient and horizontal normal stress extracted in the core were found to be very nearly the same as those obtained in periodic domain simulations at the same grid resolution, while the shear viscosity in the core was about $15 \%$ higher than that estimated in periodic domain simulations. These confirm that 
periodic domain simulations capture to a good accuracy the mesoscale structures in the core. In these figures, the results are shown for one-half of the channel width, and the distance is measured from the wall. It is readily seen that all 3 filtered quantities are significantly different in the core and the wall regions; for example, the filtered drag coefficient from the core region of the suspension is about $\sim 5$ times larger than the filtered drag coefficient at the wall. The error bars showing the uncertainty in wall corrections are larger than the difference between the curves corresponding to four different particle concentrations (inside filtering regions); therefore, we can conclude that the pattern seen in this figure is essentially independent of the mean particle phase volume fraction in a filtering region. It can also be inferred that conditions outside the filtering region do not affect the statistical averages of consequences resulting from the mesoscale structures inside the filtering region; as a result, simple algebraic closure models for the drag coefficient, particle phase horizontal normal stress and shear viscosity suffice.

The dependence of the filtered closures on the distance from the walls may be rationalized as follows: it is entirely reasonable that fluctuations will be dampened in the vicinity of solid boundaries, and as a result the filtered horizontal normal stress should be diminished near the boundaries (just as in single phase turbulent flows ${ }^{52}$ ). (As the fluctuations associated with the mesoscale structures contribute to breakup of clusters, diminished fluctuations near the boundaries result in larger clusters and hence lower drag coefficient. The wall effect seen in the filtered horizontal normal stress and drag coefficient appear to extend to approximately the same distance away from the wall, while that for the shear viscosity effect is seen to persist for a little further away from the wall.

Having ascertained that the wall corrections to the filtered closures are nearly independent of the mean volume fraction in the filtering region, we performed the rest of the 
analysis by aggregating the results obtained at various filtered particle phase volume fractions. The effect of the filter length on the wall corrections to the filtered drag coefficient, filtered particle phase horizontal normal stress and shear viscosity are shown in Figures 4a-c, respectively, for three filter lengths $(2.056,4.112$, and $8.224 d u)$. All three quantities have been scaled by the respective values in the core region, which depends systematically on filter length; however, the scaled quantities presented in these figures are nearly independent of filter length (to within the error bars shown). In other words, the filter length dependence of the filtered closures is essentially independent of the distance from the wall.

Figure $5 \mathrm{a}$ to $5 \mathrm{c}$ display the variation of the filtered drag coefficient, particle phase horizontal normal stress and shear viscosity with the distance from the wall, extracted from the kinetic theory model simulations for three different channel widths, given in the figure legend. (For each width, the results are shown for one-half of the channel width.) All the filtered quantities have been scaled with the corresponding quantities extracted from the core region (which were found to be nearly the same for all three channel widths). The results obtained with different channel widths nearly collapse (to within the confidence limit indicated by the error bars), clearly indicating that the wall effect should not be measured as a fraction of the channel width; instead it should be viewed in terms of actual distance (or made dimensionless using a length scale other than the channel width) from the wall.

In the figures presented above, we scaled the distance with $\mathrm{v}_{t}^{2} / g$, which is the same scale we used to make filter length dimensionless. To ascertain that this scaling is appropriate, we performed simulations with two different particle diameters $(75$ and $100 \mu \mathrm{m})$. The lines in Figure $5 \mathrm{a}$ to $5 \mathrm{c}$ were generated using the $75 \mu \mathrm{m}$ particles, while the squares were obtained for 
$100 \mu \mathrm{m}$ particles. For both sets, the variation of the filtered closures with distance from the channel wall collapsed onto the same curves confirming that the scaling is indeed appropriate.

We then investigated the effect of flow conditions, such as particle mass flux and gas velocity, and the wall boundary conditions (particle-wall restitution coefficient and specularity coefficient for the Johnson and Jackson boundary condition) on the wall corrections to the filtered quantities. Among these, only wall-particle specularity coefficient had a measurably significant effect (in the 2D system studied here).

To study the sensitivity of the wall corrections to the specularity coefficient $\varphi$, we carried out simulations for 5 different values of $\varphi: 0,0.0001,0.3,0.6$ and 1 in a channel with a width of $102.8 \mathrm{du}$. Snapshots of the particle phase volume fraction field extracted from two of these simulations (for $\varphi=0.0001$ and 0.6 ) are shown in Figure 2. It has been reported that $\varphi$ has a significant effect on the particle concentration near the wall; more specifically, a lower specularity coefficient yields higher particle concentration near the wall. ${ }^{46,47,49}$ This is indeed what we see in these snapshots; particles tend to accumulate more and more as the specularity coefficient reaches free slip limit. It is also apparent that strands and clusters in the wall vicinity orient themselves in the vertical direction more for the lower specularity coefficient values. Figures 6a to $6 \mathrm{c}$ display the variation of scaled filtered drag coefficient, particle phase horizontal normal stress and shear viscosity with the distance from the wall for various $\varphi$ values. It is clear that the value of $\varphi$ has a quantitative effect on the wall corrections, but the dependence on $\varphi$ nearly vanishes for $\varphi>0.6$.

The correlations summarized in Table 2 capture the results on wall corrections to the filtered model closures and the specularity coefficient dependence described above. 


\section{Wall boundary conditions for the filtered equations}

It is generally believed that in high-velocity flows of densely loaded gas-particle mixtures through large risers the vertical pressure gradient is largely due to the particle hold-up and the wall shear is only weakly relevant. ${ }^{53}$ To test this, we calculated wall shear stress per unit height (under SSS conditions) from highly-resolved kinetic theory based two-fluid model simulations (with $\varphi=0.6$ ) for two different channel widths, 61.68 and $102.8 \mathrm{du}$ and found that wall shear supported $\sim 3 \%$ and $\sim 1 \%$ of the weight of the particles, respectively. When $\varphi$ was lowered to 0.0001 in the $61.68 \mathrm{du}$ wide channel, the wall shear stress supported only $0.003 \%$ of the weight of the particles. These comparisons confirm that the wall shear stress is considerably small compared to the weight of the particles in the channel and that the primary role of the wall is the "no penetration condition." With this in mind, we have set in the remainder of this study the wall boundary condition (BC) for the filtered equations at the bounding walls in $2 \mathrm{D}$ systems as free slip for the particle phase. The observation from Figure $4 \mathrm{c}$ that the filtered shear viscosity in the immediate vicinity of the walls is considerably smaller than that for the bulk region (at a comparable particle volume fraction) provides further support for such simple BCs.

One can readily extrapolate the above analysis for the particle phase to the gas phase. We simply note here that the effective BC for the gas phase approaches free slip as the filter length increases, just as in the case of the particle phase. Thus, for modestly large filters (of the order of a few cms for the FCC particles mentioned in Table 1), one can use free slip BC for both phases as a good first approximation in 2D simulations. Here, it should be noted that if the tube diameter (or channel width) is small, the wall effect is expected to become more important and free slip BC may not be a good choice for wall boundaries. However, the filtered models will not be necessary for these computationally affordable simulations. 


\section{Grid resolution dependence of channel flow simulations}

Figures 7a to 7c show snapshots in the SSS for kinetic theory simulations (of gas-particle flows in a 2D channel as in Figure 1) at three different grid resolutions, while figures $7 \mathrm{~d}$ to $7 \mathrm{f}$ show simulations with a filtered model corresponding to a filter length of $4.112 d u$ (and including wall corrections). It is readily apparent that finer and finer structures got resolved with increasing grid resolution for the kinetic theory cases, while this was not the case with filtered model simulations.

Figure 8 shows the variation of the time-averaged (scaled) particle phase mass inventory with grid resolution, determined from the simulations with the kinetic theory and the filtered model with and without wall corrections. (Although we had established that wall corrections to the closures in filtered model are appreciable, we performed filtered model simulations with and without wall corrections to learn more about the extent of influence brought about by the wall corrections.) The particle mass inventory was scaled with the product of the particle density and the volume of the bed. The grid resolution increases from left to right. It is readily seen that the particle mass inventory predicted by the kinetic theory model, indicated with light gray squares, became only grid resolution independent when the grid length was smaller than $1.028 \mathrm{du}$. However, an inspection of the lateral particle volume fraction and mass flux profiles (not shown) revealed that this grid length was not sufficient to obtain grid resolution independent results and that the kinetic theory model did not show any concrete evidence of converging even at the highest resolution affordable.

In the case of filtered model simulations with wall corrections, represented by black diamond-shaped symbols in Figure 8, as the grid resolution was increased, the particle phase 
mass inventory increased initially, but essentially became grid resolution independent once the grid length became smaller or equal to $\sim 2.056 \mathrm{du}$, which is one-half of the filter length.

Figure 9a compares the variation of temporally and laterally averaged particle volume fraction (in the SSS) with the elevation in the 2D channel obtained from simulations of the filtered model (including wall corrections) for four grid resolutions. At the bottom of the riser and near the top, the particle volume fraction changes rapidly, indicating strong entrance and end effects; however, in the middle region, the variation is gradual; the average particle phase volume fractions decreases with increasing elevation. The variation pattern is similar for all grid resolutions at all elevations. It is clear from the figure that the two uppermost curves, representing the average particle volume fraction profile determined with grid lengths of 1.028 and $2.056 \mathrm{du}$, essentially overlap. Figures $9 \mathrm{~b}$ to $9 \mathrm{~d}$ show the corresponding variations of the time-averaged particle phase volume fraction, particle mass flux and gas velocity (respectively) with dimensionless distance from the bounding wall at an elevation of $616.8 \mathrm{du}$ for three different dimensionless grid lengths. At all three resolutions, particles accumulate in the vicinity of the walls, and a dilute core region is observed. It is clear from these figures that lateral profiles are nearly identical for grid lengths of 1.028 and $2.056 \mathrm{du}$. Similar results were obtained at other elevations away from the entrance and exit effects. Figure 9c includes the time-averaged particle mass flux results obtained from the most highly resolved kinetic theory model simulations done in this study. The agreement between the filtered and kinetic theory models is good, clearly indicating the filtered model solutions do indeed correspond to the kinetic theory model from which they were developed. This is required of a successful filtered model and it serves as a verification of the filtered model approach. Additional such verifications are provided later in this manuscript. 
In the above examples, we employed free slip BCs for the gas and particle phases at all bounding walls. If one replaces the free slip conditions with no-slip for both phases, the mass holdup predicted by the filtered model decreased by $\sim 30 \%$ (see figure 8 ). These two extremes (free slip and no slip) serve as bounds and thus give an idea about the extent of the changes in the mean flow characteristics that can come about altering the boundary conditions. In this example, the free slip boundary conditions led to better match with the kinetic theory results, for reasons discussed earlier.

Figure 8 also shows the results obtained when the wall corrections were turned off in the filtered model (while using free slip BCs). Nearly grid resolution independent solution was still achieved as when the wall corrections were included; however, a much smaller mass inventory was predicted without wall corrections leading to quantitatively very different results from that obtained with the highly resolved kinetic theory model simulation. This illustrates that wall corrections are important for quantitative accuracy. (We also explored the effect of including the wall corrections to the filtered drag coefficient, particle phase horizontal normal stress and shear viscosity one at a time or two at a time on the predictions of the filtered model equations. This analysis revealed that the corrections affected the results predicted by the filtered model simulations quantitatively - namely, increase or decrease particle phase mass inventory in the channel, but did not affect the grid resolution independence of these results. Quantitative comparison with the kinetic theory results were obtained only when all three corrections were included.) 


\section{Grid resolution requirement for filtered models}

As the closures for the filtered model were derived by averaging over structures smaller than a chosen filter length, $\Delta_{f}$, it is reasonable to demand that solution of the filtered model should not yield fine structures much smaller than the filter length; if it did, it suggests weakness in the model formulation. Thus, for a satisfactorily constructed filtered model, the grid length, $\Delta_{g}$, required for grid-independent solutions should scale with the filter length. The results

presented in Figures 8 and 9 suggest that $\Delta_{g} \sim 0.5 \Delta_{f}$ is essentially adequate. With this in mind, we propose that the grid length be set as $\Delta_{g} \sim 0.3-0.5 \Delta_{f}$ in filtered model simulations

Addition of a particle phase bulk viscosity term to the filtered model

In the filtered model, the particle phase stress was modeled as follows:

$$
\Sigma_{s}=\overline{p_{s, \text { fittered }}} \mathbf{I}-\overline{\mu_{b, \text { filtered }}}(\nabla \cdot \overline{\mathbf{v}}) \mathbf{I}-\overline{\mu_{s, \text { filtered }}}\left(\nabla \overline{\mathbf{v}}+(\nabla \overline{\mathbf{v}})^{\mathrm{T}}-\frac{2}{3}(\nabla \cdot \overline{\mathbf{v}}) \mathbf{I}\right)
$$

where $\overline{\mu_{s, \text { filtered }}}$ and $\overline{\mu_{b, \text { filtered }}}$ are the shear and bulk viscosities, respectively. As we did not determine $\overline{\mu_{b, \text { fitered }}}$ by filtering the results from highly resolved kinetic theory model simulations, we had set it to zero in the examples presented above. We repeated the simulations in Figure 9 while setting the filtered bulk viscosity to be the same as the filtered shear viscosity, and found virtually no change. It is therefore reasonable to conclude that a bulk viscosity term in the filtered model is not important for channel flows. We also studied the effect of adding $a$ mesoscale viscosity term to the gas phase filtered model equations and found it to have a very weak effect (if any) on the channel flow results predicted by the filtered model simulations. 


\section{The effect of filter size on predictions of the filtered models}

Snapshots of particle volume fraction fields in the SSS for gas-particle flow in vertical 2D channel (whose width is $102.8 \mathrm{du}$ ) obtained by solving filtered models with filter lengths of 4.112 and $8.224 d u$ are shown in Figures 10a and 10b, respectively. The simulation with coarser filter yielded slightly coarser structures, as expected.

The variation of temporally and laterally averaged particle volume fraction with elevation in the channel, obtained in the SSS of these two simulations is presented in Figure 11(a). It is apparent that both filtered models yielded nearly identical results. The average particle phase mass inventory scaled with the product of the channel volume and particle density was computed to be 0.135 and 0.136 for the filtered models with filter lengths of 4.112 and $8.224 d u$, respectively.

Figures $11 \mathrm{~b}$ to $11 \mathrm{~d}$ present the variation of the time-averaged particle phase volume fraction, axial particle phase mass flux, and axial superficial gas velocity with the distance from the wall at two elevations, obtained from the two filtered models; once again the agreement is good. In all the results presented in Figures 10 and 11 we have used the same grid length of $2.056 \mathrm{du}$, which is one half the size of the smaller filter used in the comparison. We simply note that when the filtered model with the larger filter was repeated using a grid length of $4.112 \mathrm{du}$, virtually the same results were obtained; so the grid length recommendation made earlier still applies.

These observations that filtered models corresponding to two different filter lengths (4.112 and $8.224 \mathrm{du}$ ) yielded comparable results provide support for the soundness of the approach. 


\section{Further verification of the filtered model}

Earlier in this manuscript, we provided one comparison between the results obtained with a filtered two-fluid model and (highly resolved simulations of) the kinetic theory based two-fluid model, for the case of a very bumpy wall (with $\varphi=0.6$ ). We now consider the case of a much smoother wall with $\varphi=0.0001$. Snapshots of the particle volume fraction field (in the SSS) from the kinetic model simulations with grid lengths of $0.514,1.028$, and $4.112 d u$ and the filtered model simulation (with filter and grid lengths of $4.112 \mathrm{du}$ and $1.028 \mathrm{du}$, respectively) are shown in Figures 12a to 12d. As shown earlier in Figure 7, the kinetic theory model simulations yielded finer and finer structures as the grid length decreased.

Figure 13 compares the variation of temporally and laterally averaged particle volume fraction with the elevation in the $2 \mathrm{D}$ channel obtained from simulations of the kinetic theory model for four grid resolutions and that from the filtered model simulations with a grid length of $1.028 \mathrm{du}$. The grid resolution dependence of the kinetic theory model is clearly seen in the figure. The filtered model profile is slightly above the kinetic model simulation with $0.514(d u)$ grids and appears to provide an asymptotic value for the results that would be predicted by the kinetic theory model (at an even finer resolution).

The variation of the time-averaged particle phase volume fraction, dimensionless axial particle phase mass flux and superficial gas velocity (predicted by both models) with the dimensionless distance from one of the vertical bounding walls are presented in Figures 14 to 16, respectively, with panels a and $\mathrm{b}$ in each figure corresponding to two different elevations. These figures clearly show the grid resolution dependence of the results predicted by the kinetic theory model. It is also apparent in the figures that the results predicted by the filtered model and the kinetic model (with $0.514 \mathrm{du}$ grids) are comparable. (The results obtained with the filtered model 
for a grid length of $2.056 \mathrm{du}$ are very close to that reported in these figures and hence are not shown.) These figures lend further credence to the filtered model approach to obtaining solutions for such flow problems.

\section{CPU time comparison}

Figure 17 shows the CPU times required to compute one second of flow for the kinetic theory based two-fluid (gray squares) and the filtered model with a filter length of $4.112 \mathrm{du}$ (black circles) at various grid resolutions. All the simulation conditions are described in the figure caption (and in Figure 7). The simulations reported in this were performed on Dual quad core Intel Xeon E5420 processors running at $2.50 \mathrm{GHz}$. The filtered-model based simulation with a grid length of $1.028 d u$ ran $\sim 4$ times faster than the kinetic theory based simulation at the same resolution. This can be attributed to the finer structures contained in the kinetic theory model, see Figure 7. Note that one does not need to use such a small $1.028 \mathrm{du}$ grid length with the filtered model; when the grid length in the filtered model simulation is increased to $2.056 \mathrm{du}$, it ran $\sim 30$ and 300 times faster than the kinetic theory based two-fluid model with grid lengths of 1.028 and $0.514 d u$, respectively. When the specularity coefficient was changed to 0.0001 , the filtered-model simulation with filter and grid lengths of 4.112 and $1.028 d u$ (respectively) ran $\sim 5$ and 40 times faster than the kinetic theory based simulation with grid lengths of $1.028 \mathrm{du}$ and $0.514 d u$, respectively. (Once again, a grid length of $2.056 d u$ would have sufficed for this filtered model, which would have lowered the computational time for the filtered model by a factor of $\sim 7.5$.)

Next, we compared the simulation times (for flow in the $102.8 \mathrm{du}$-wide channel) for filtered models with two different filter lengths and a grid size of $2.056 \mathrm{du}$. The model with a filter length of $8.224 d u$ ran 3 times faster than that with a filter length of $4.112 \mathrm{du}$. This can be 
attributed to the finer structures contained in the latter model. Note that one does not need to use a grid length of $2.056 \mathrm{du}$ for a model with a filter length of $8.224 \mathrm{du}$; the filtered model with a filter length of $8.224 d u$ and grid lengths of 2.570 and 4.112 ran $\sim 7$ and 25 times faster than the filtered model with a filter length of $4.112 \mathrm{du}$ and a grid length of $2.056 \mathrm{du}$. Combining this example with the one in the previous paragraph, we project that a filtered model with a filter length of $8.224 d u$ (and $4.112 d u$ grids) will yield solution $\sim 7500$ times faster than the kinetic theory model with a grid length of $0.514 d u$ (which was seen to be necessary to get nearly grid independent solution).

For the $75 \mu \mathrm{m}$ particles in Table 1, a filter length of $8.224 d u$ translates to a filter length of $4 \mathrm{~cm}$. In large scale processes, one typically uses even larger grids and so one would use even larger filters and correspondingly faster simulations (when compared to highly resolved kinetic theory model simulations whose required grid resolution is not likely to change with process vessel size). Finally, when one considers 3D simulations, the difference between filtered and well-resolved kinetic theory models is expected to be even larger.

In summary, filtered models allow us to study the large structures in gas-particle flows without having to resolve smaller structures and in a computationally affordable and faster manner.

\section{Summary}

We have investigated the effect of the bounding walls on the closure relationships for the filtered two-fluid model equations through a set of 2D flow simulations in a channel equipped with bounding walls and inlet and outlet regions. The present study, which is valid for the particle volume fraction range typical of gas-particle flows in risers, reveals that: 
a. Closures for the filtered drag coefficient and particle phase stress depend not only on particle volume fraction and the filter length, but also on the distance from the wall.

b. The wall effect should not be measured as a fraction of the channel width; instead, it should be viewed in terms of the actual distance from the wall. The characteristic length to scale the distance from the wall is the same as that used to scale the filter length.

c. The wall corrections to the filtered closures are nearly independent of the mean particle volume fraction in the filtering region.

d. The wall corrections to the filtered closures are nearly independent of the filter lengths considered in this study.

e. The simplest effective BC for the filtered equations at the bounding walls is free slip BC.

Filtered model simulations performed in this study revealed the following:

a. Grid resolution independent solutions resulted when the grid length is $\sim$ half the filter length or smaller.

b. Filtered models did indeed yield the same coarse statistical results as highly resolved simulations of kinetic theory based two-fluid model (which was used to derive the filtered models).

c. Filtered models with two different filter lengths were shown to afford the same coarse statistical results.

d. Filtered model simulations required significantly less computational time when compared to highly resolved kinetic theory model simulations. 
The present study establishes the fidelity of the filtered model, but two important tasks remain: (a) It would be useful to establish general closures for the filtered model in terms of particle volume fraction and filter size so that they can be broadly used by researchers; (b) The filtered model predictions should be compared against experimental data. These will be described in future publications.

\section{Acknowledgments}

This work was supported by the US Department of Energy (grants: CDE-FC2600NT40971 and DE-PS26-05NT42472-11) and the ExxonMobil Research \& Engineering Company. Igci acknowledges summer training on MFIX at the National Energy Technology Laboratory, Morgantown, WV. We thank Sreekanth Pannala, Madhava Syamlal, Chris Guenther, Ronald Breault and Sofiane Benyahia for their assistance throughout the course of this study. 


\section{Nomenclature}

$d_{p} \quad$ Particle diameter (m)

$e_{p} \quad$ Coefficient of restitution for particle-particle collisions

$e_{w} \quad$ Coefficient of restitution for wall-particle collisions

$\mathrm{Fr}_{f} \quad$ Froude number based on filter length $=\mathrm{v}_{\mathrm{t}}^{2} / g \Delta_{f}$

$\mathrm{Fr}_{g} \quad$ Froude number based on grid length $=\mathrm{v}_{\mathrm{t}}^{2} / g \Delta_{g}$

$g, \mathbf{g} \quad$ Acceleration due to gravity $\left(\mathrm{m} / \mathrm{s}^{2}\right)$

$g_{o} \quad$ Value of radial distribution function at contact

$\overline{p_{s, \text { filtered }}} \quad$ Filtered particle phase pressure $\left(\mathrm{kg} / \mathrm{m} \cdot \mathrm{s}^{2}\right)$

$\overline{p_{s, \text { filtered }, d}} \quad \overline{p_{s, \text { filtered }, d}}$ made dimensionless; $\overline{p_{s, \text { filtered }, d}}=\overline{p_{s, \text { filtered }}} / \rho_{s} \mathrm{v}_{\mathrm{t}}^{2}$

$\overline{p_{s, \text { filtered }, \mathrm{xx}}} \quad$ Filtered particle phase horizontal normal stress $\left(\mathrm{kg} / \mathrm{m} . \mathrm{s}^{2}\right)$

$\overline{p_{s, \text { filtered, } \mathrm{xx}, \text { core }}} \quad \overline{p_{s, \text { filtered }, \mathrm{xx}}}$ extracted from the core region of the $2 \mathrm{D}$ channel $\left(\mathrm{kg} / \mathrm{m} . \mathrm{s}^{2}\right)$

$\overline{p_{s, \text { filtered }, \mathrm{xx}, \text { periodic }}} \overline{p_{s, \text { filtered,xx }}}$ extracted from periodic BC simulations $\left(\mathrm{kg} / \mathrm{m} . \mathrm{s}^{2}\right)$

$\overline{p_{s, \text { filtered }, \mathrm{xx}, d}} \quad \overline{p_{s, \text { filtered }, \mathrm{xx}, d}}$ made dimensionless; $\overline{p_{s, \text { filtered }, \mathrm{xx}, d}}=\overline{p_{s, \text { filtered }, \mathrm{xx}}} / \rho_{s} \mathrm{v}_{\mathrm{t}}^{2}$

$\overline{p_{s, \text { filtered, } \mathrm{xx}, \text { scaled }}} \quad \overline{p_{s, \text { filtered } \mathrm{xx}}}$ scaled with $\overline{p_{s, \text { filtered }, \mathrm{xx}, \text { core }}} ; \overline{p_{s, \text { filtered, } \mathrm{xx}, \text { scaled }}}=\overline{p_{s, \text { filtered, } \mathrm{xx}}} / \overline{p_{s, \text { filtered, } \mathrm{xx}, \text { core }}}$

$\overline{p_{s, k i n e t i c}} \quad$ Filtered value of particle phase pressures in the kinetic theory model $\left(\mathrm{kg} / \mathrm{m} . \mathrm{s}^{2}\right)$

q Flux of granular energy $\left(\mathrm{kg} / \mathrm{s}^{3}\right)$

$\mathrm{v}_{\mathrm{t}} \quad$ Terminal settling velocity $(\mathrm{m} / \mathrm{s})$

V Particle phase velocity in the microscopic two-fluid model (m/s)

$\overline{\mathbf{v}} \quad$ Filtered particle phase velocity $(\mathrm{m} / \mathrm{s})$ 
Greek Symbols

$\overline{\beta_{\text {filtered }}}$

$\overline{\beta_{\text {filtered,core }}}$

$\overline{\beta_{\text {filtered, periodic }}}$

$\overline{\beta_{\text {filtered }, d}}$

$\overline{\beta_{\text {filtered,scaled }}}$

$\phi_{s}, \phi_{g}$

$\phi_{\mathrm{s}, \max }$

$\overline{\phi_{s}}, \overline{\phi_{g}}$

$\rho_{s}, \rho_{g}$

$\Delta_{f}$

$\Delta_{g}$

$\underline{\underline{\sigma_{s}}}$

$\Sigma_{\text {s }}$
Filtered drag coefficient $\left(\mathrm{kg} / \mathrm{m}^{3} . \mathrm{s}\right)$

$\overline{\beta_{\text {filtered }}}$ extracted from the core region of the $2 \mathrm{D}$ channel $\left(\mathrm{kg} / \mathrm{m}^{3} . \mathrm{s}\right)$

$\overline{\beta_{\text {filtered }}}$ extracted from periodic BC simulations $\left(\mathrm{kg} / \mathrm{m}^{3} . \mathrm{s}\right)$

Dimensionless filtered drag coefficient $=\overline{\beta_{\text {filtered }}} \mathrm{V}_{\mathrm{t}} / \rho_{s} g$

$\overline{\beta_{\text {filtered }}}$ scaled with $\overline{\beta_{\text {filtered,core }}} ; \overline{\beta_{\text {filtered,scaled }}}=\overline{\beta_{\text {filtered }}} / \overline{\beta_{\text {filtered,core }}}$

Particle and gas phase volume fractions, respectively

Maximum particle volume fraction

Filtered particle and gas phase volume fractions, respectively

Particle and gas densities, respectively $\left(\mathrm{kg} / \mathrm{m}^{3}\right)$

Filter length (m)

Grid length (m)

Particle phase stress tensor in the kinetic theory model $\left(\mathrm{kg} / \mathrm{m} . \mathrm{s}^{2}\right)$

Filtered total particle phase stress $\left(\mathrm{kg} / \mathrm{m} . \mathrm{s}^{2}\right)$ 
$\mu_{s}$

$\mu_{b, \text { filtered }}, \mu_{s, \text { filtered }}$

$\mu_{s, \text { filtered }, d}$

$\mu_{s, \text { filtered,core }}$

$\mu_{\text {s, filtered,periodic }}$

$\mu_{\text {s, filtered,scaled }}$

$\Theta_{s}$

$\varphi$
Granular thermal conductivity (kg/m.s)

Gas phase viscosity (kg/m.s)

Shear viscosity of the particle phase appearing in the kinetic theory model $(\mathrm{kg} / \mathrm{m} . \mathrm{s})$

Bulk and shear viscosities of the particle phase appearing in the filtered two-fluid model (kg/m.s)

$\overline{\mu_{s, \text { filtered }}}$ made dimensionless; $\overline{\mu_{s, \text { filtered }, d}}=\overline{\mu_{s, \text { filtered }}} g / \rho_{\mathrm{s}} \mathrm{v}_{\mathrm{t}}^{3}$

$\overline{\mu_{s, \text { filtered }}}$ extracted from the core region of the $2 \mathrm{D}$ channel $(\mathrm{kg} / \mathrm{m} . \mathrm{s})$

$\overline{\mu_{s, \text { filtered }}}$ extracted from periodic BC simulations ( $\left.\mathrm{kg} / \mathrm{m} . \mathrm{s}\right)$

$\overline{\mu_{s, \text { filtered }}}$ scaled with $\overline{\mu_{s, \text { filtered,core }}} ; \overline{\mu_{s, \text { filtered,scaled }}}=\overline{\mu_{s, \text { filtered }}} / \overline{\mu_{s, \text { filtered,core }}}$

Granular temperature $\left(\mathrm{m}^{2} / \mathrm{s}^{2}\right)$

Wall specularity coefficient 


\section{Literature Cited}

1. Schnitzlein MG, Weinstein H. Flow Characterization in High-Velocity Fluidized Beds Using Pressure Fluctuations. Chem Eng Sci. 1988;43:2605-2614.

2. Gidaspow D. Multiphase Flow and Fluidization. San Diego, CA, CA: Academic Press; 1994.

3. Nieuwland JJ, Annaland MV, Kuipers JAM, van Swaaij WPM. Hydrodynamic Modeling of Gas/Particle Flows in Riser Reactors. AIChE J. 1996;42:1569-1582.

4. Neri A, Gidaspow D. Riser hydrodynamics: Simulation using kinetic theory. AIChE J. 2000;46:52-67.

5. Weinstein H, Shao M, Schnitzlein M. Radial variation in solid density in high velocity fluidization. In: Basu P, ed. Circulating Fluidized Bed Technology. Elmsford, NY: Pergamon; 1986:201.

6. Bader R, Findlay J, Knowlton T. Gas/Solid flow patterns in a 30.5-cm diameter circulating fluidized bed. In: Basu P, Large JF, eds. Circulating Fluidized Bed Technology. Vol II. Elmsford, NY: Pergamon; 1988:123-137.

7. Chen JC. Clusters. AIChE Sym S. 1996;92:1-5.

8. Sharma AK, Tuzla K, Matsen J, Chen JC. Parametric effects of particle size and gas velocity on cluster characteristics in fast fluidized beds. Powder Technol. 2000;111:114122.

9. Harris AT, Davidson JF, Thorpe RB. The prediction of particle cluster properties in the near wall region of a vertical riser (200157). Powder Technol. 2002;127:128-143.

10. Jackson R. The Dynamics of Fluidized Particles: Cambridge University Press; 2000. 
11. Syamlal M, Rogers W, O'Brien TJ. MFIX Documentation. Morgantown, WV: U.S. Department of Energy, Federal Energy Technology Center;1993.

12. Agrawal K, Loezos PN, Syamlal M, Sundaresan S. The role of meso-scale structures in rapid gas-solid flows. J Fluid Mech. 2001;445:151-185.

13. Wang J. High-resolution Eulerian simulation of RMS of solid volume fraction fluctuation and particle clustering characteristics in a CFB riser. Chem Eng Sci. 2008;63:3341-3347.

14. Wang J, van der Hoef MA, Kuipers JAM. Why the two-fluid model fails to predict the bed expansion characteristics of Geldart A particles in gas-fluidized beds: A tentative answer. Chem Eng Sci. 2009;64:622-625.

15. Sundaresan S. Modeling the Hydrodynamics of Multiphase Flow Reactors: Current Status and Challenges. AIChE J. 2000;46:1102-1105.

16. Qi H, Li F, You C. Modeling of drag with the Eulerian approach and EMMS theory for heterogeneous gas-solid two-phase flow. Chem Eng Sci. 2007;62:1670-1681.

17. O'Brien TJ, Syamlal M. Particle cluster effects in the numerical simulation of a circulating fluidized bed. In: Avidan A, ed. Circulating Fluidized Bed Technology. IV. Proceedings of the Fourth International Conference on Circulating Fluidized Beds. Hidden Valley Conference Center, Somerset, PAAugust 1-5, 1993.

18. Boemer A, Qi H, Hannes J, Renz U. Modelling of solids circulation in a fluidized bed with Eulerian approach. Paper presented at: 29th IEA-FBC MeetingNov. 24-26, 1994; Paris, France.

19. Heynderickx GJ, Das AK, De Wilde J, Marin GB. Effect of Clustering on Gas-Solid Drag in Dilute Two-Phase Flow. Ind Eng Chem Res. 2004;43:4635-4646. 
20. McKeen T, Pugsley T. Simulation and experimental validation of a freely bubbling bed of FCC catalyst. Powder Technol. 2003;129:139-152.

21. Yang N, Wang W, Ge W, Wang L, Li J. Simulation of Heterogeneous Structure in a Circulating Fluidized-Bed Riser by Combining the Two-Fluid with the EMMS Approach. Ind Eng Chem Res. 2004;43:5548-5561.

22. Yang N, Wang W, Ge W, Li J. CFD simulation of concurrent-up gas-solid flow in circulating fluidized beds with structure dependent drag coefficient. Chem Eng J. 2003;96:71-80.

23. Li J, Kwauk M. Exploring complex systems in chemical engineering - the multi-scale methodology. Chem Eng Sci. 2003;58:521-535.

24. Wang J, van der Hoef MA, Kuipers JAM. Coarse grid simulation of bed expansion characteristics of industrial-scale gas-solid bubbling fluidized beds. Chem Eng Sci. 2010;65:2125-2131.

25. Krishna R, van Baten JM. Using CFD for scaling up gas-solid bubbling fluidized bed reactors with Geldart A powders. Chem Eng J. 2001;82:247-257.

26. Dasgupta S, Jackson R, Sundaresan S. Turbulent gas-particle flow in vertical risers. AIChE J. 1994;40:215-228.

27. Hrenya CM, Sinclair JL. Effects of Particle-Phase Turbulence in Gas-Solid Flows. AIChE J. 1997;43:853-869.

28. Andrews IV AT, Loezos PN, Sundaresan S. Coarse-Grid Simulation of Gas-Particle Flows in Vertical Risers. Ind Eng Chem Res. 2005;44:6022-6037.

29. Igci Y, Andrews IV AT, Sundaresan S, Pannala S, O'Brien T. Filtered Two-Fluid Models for Fluidized Gas-Particle Suspensions. AIChE J. 2008;54:1431-1448. 
30. Zhang DZ, VanderHeyden WB. The effects of mesoscale structures on the macroscopic momentum equations for two-phase flows. Int J Multiphas Flow. 2002;28:805-822.

31. Li J. Multi-scale modeling and method of energy minimization. Beijing1987.

32. Li J, Tung Y, Kwauk M. Energy Transport and regime transition of particle-fluid twophase flow. In: Basu P, Large JF, eds. Circulating fluidized bed technology II. Oxford: Pergamon Press; 1988:75.

33. Li J, Cheng C, Zhang Z, Yuan J. The EMMS model, its application, development and updated concepts. Chem Eng Sci. 1999;54:5409-5425.

34. Li J, Zhang Z, Ge W, Sun Q, Yuan J. A simple variation criterion for turbulent flow in pipe. Chem Eng Sci. 1999;54:1151-1154.

35. Li J, Kwauk M. Particle-fluid two-phase flow energy-minimization multi-scale method. Beijing: Metallurgical Industry Press; 1994.

36. Wang J, Ge W. Multi-scale analysis on particle-phase stresses of coarse particles in bubbling fluidized beds. Chem Eng Sci. 2006;61:2736-2741.

37. Lu B, Wang W, Li J, et al. Multi-scale CFD simulation of gas-solid flow in MIP reactors with a structure-dependent drag model. Chem Eng Sci. 2007;62:5487-5494.

38. Wang W, Li J. Simulation of gas-solid two-phase flow by a multi-scale CFD approachExtension of the EMMS model to the sub-grid level. Chem Eng Sci. 2007;62:208-231.

39. Chalermsinsuwan B, Piumsomboon P, Gidaspow D. Kinetic theory based computation of PSRI riser: Part I—Estimate of mass transfer coefficient. Chem Eng Sci. 2009;64:11951211.

40. Lu B, Wang W, Li J. Searching for a mesh-independent sub-grid model for CFD simulation of gas-solid riser flows. Chem Eng Sci. 2009;64:3437-3447. 
41. Wang $\mathrm{W}, \mathrm{Lu}$ B, Li J. Choking and flow regime transitions: Simulation by a multi-scale CFD approach. Chem Eng Sci. 2007;62:814-819.

42. Benyahia S. On the Effect of Subgrid Drag Closures. Ind Eng Chem Res. 2010;49:51225131.

43. Zou B, Li H, Xia Y, Ma X. Cluster structure in a circulating fluidized bed. Powder Technol. 1994;78:173-178.

44. Miller A, Gidaspow D. Dense, Vertical Gas-Solid Flow in a Pipe. AIChE J. 1992;38:1801-1815.

45. Johnson P, Jackson R. Frictional-collisonal constitutive relations for granular materials, with application to plane shearing. J Fluid Mech. 1987;176:67-93.

46. Behyahia S, Syamlal M, O'Brien T. Evaluation of boundary conditions used to model dilute, turbulent gas/solids flows in a pipe. Powder Technol. 2005;156:62-72.

47. Almuttar A, Taghipour F. Computational fluid dynamics of a circulating fluidized bed under various fluidization conditions. Chem Eng Sci. 2008;63:1696-1709.

48. Benyahia S, Syamlal M, O'Brien TJ. Study of the Ability of Multiphase Continuum Models to Predict Core-Annulus Flow. AIChE J. 2007;53:2549-2568.

49. He J, Simonin O. Non-equilibrium prediction of particle-phase stress tensor in vertical pneumatic conveying gas-solids flows. ASME FED. 1993;166:253-263.

50. Jiradilok V, Gidaspow D, Damronglerd S, Koves WJ, Mostofi R. Kinetic theory based CFD simulation of turbulent fluidization of FCC particles in a riser. Chem Eng Sci. 2006;61:5544-5559. 
51. Wang X, Jin B, Zhong W, Xiao R. Modeling on the Hydrodynamics of a High-Flux Circulating Fluidized Bed with Geldart Group A Particles by Kinetic Theory of Granular Flow. Energ Fuel. 2010;24:1242-1259.

52. Piomelli U, Balaras E. Wall-Layer Models for Large-Eddy Simulations. Annu Rev Fluid Mech. 2002;34:349-374.

53. Chang H, Louge M. Fluid Dynamic Similarity of Circulating Fluidized-Beds. Powder Technol. 1992;17:1293-1313. 


\section{Figures and Captions:}

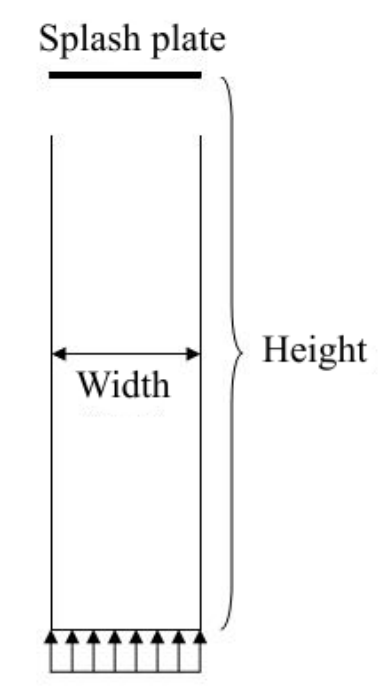

Particles in air

Figure 1: Schematic diagram of the 2D computational domain. In this set-up, the gas and particles enter the domain uniformly at the bottom. A splash plate is located over the entire width at the top. The gas and particles are allowed to leave through exit regions located on either side (just below the splash plate). The height of this opening is one-half the width of the system. 
(a)

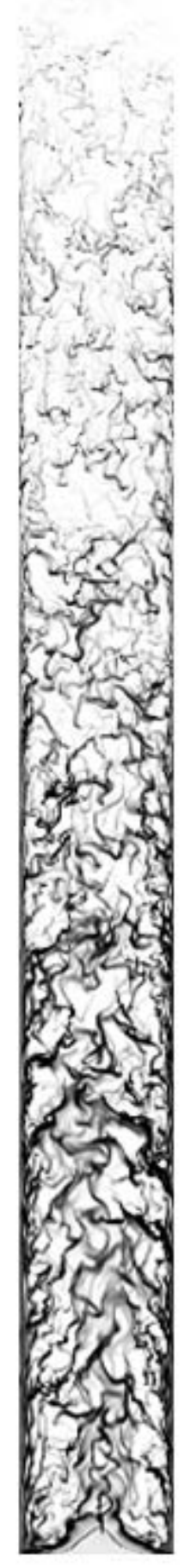

(b)

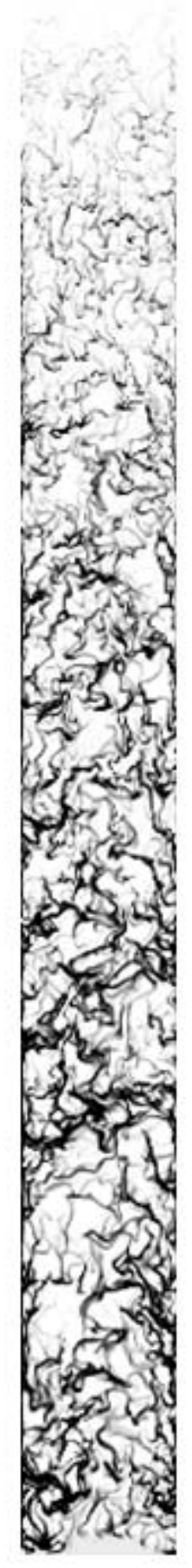


Figure 2: Snapshots of the particle phase volume fraction field extracted from the 2D simulation of the kinetic theory model equations in a 2-D domain with partial slip (Johnson and Jackson ${ }^{45}$ ) BC with (a) $\varphi=0.0001$ and (b) $\varphi=0.6$ for the particle phase and free slip BC for the gas phase at all walls. Simulation conditions: The dimensionless inlet gas and particle phase superficial velocities are 4.259 and 0.109 , respectively. The physical conditions corresponding to this simulation are listed in Table 1. The inlet particle phase volume fraction is 0.07 . Channel width and height are 102.8 and $1028 \mathrm{du}$, respectively. The gray scale axis ranges from $\phi_{s}=0.00$ (white) to $\phi_{\mathrm{s}}=0.45$ (black). 
a)

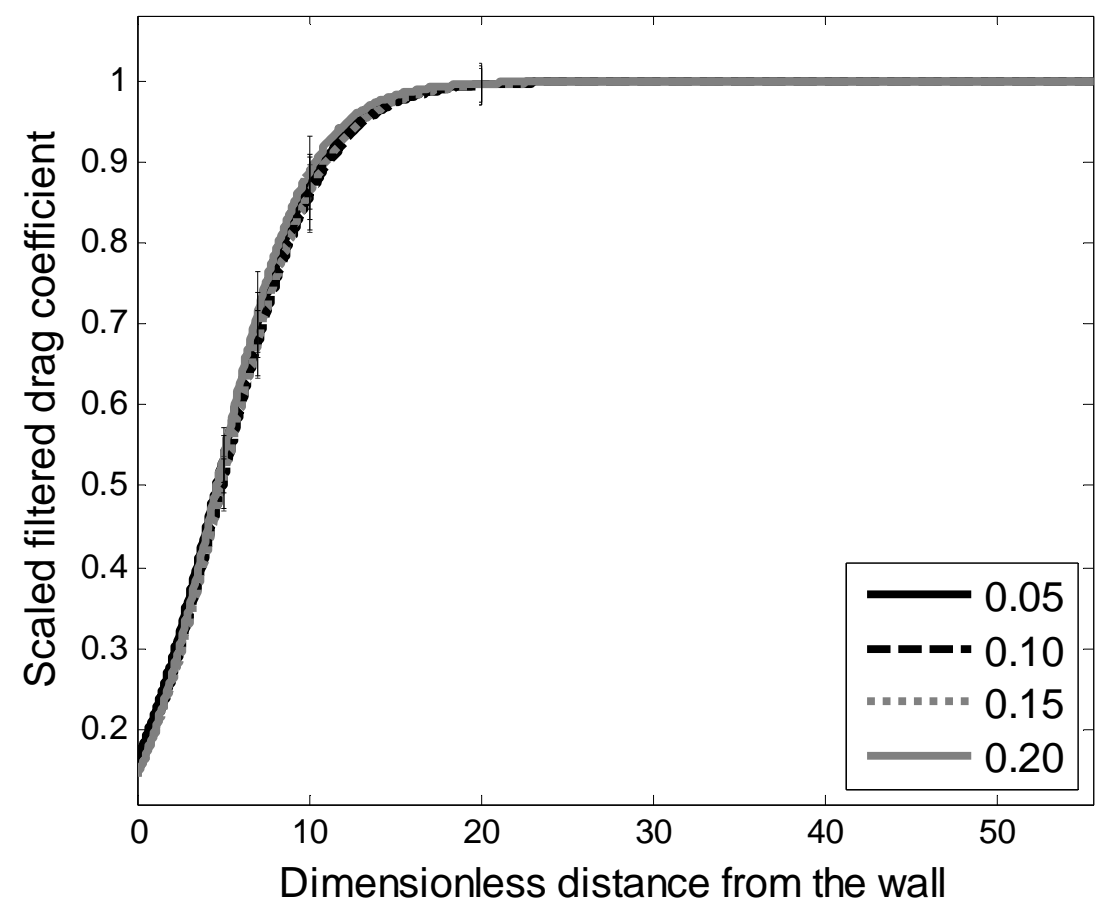

b)

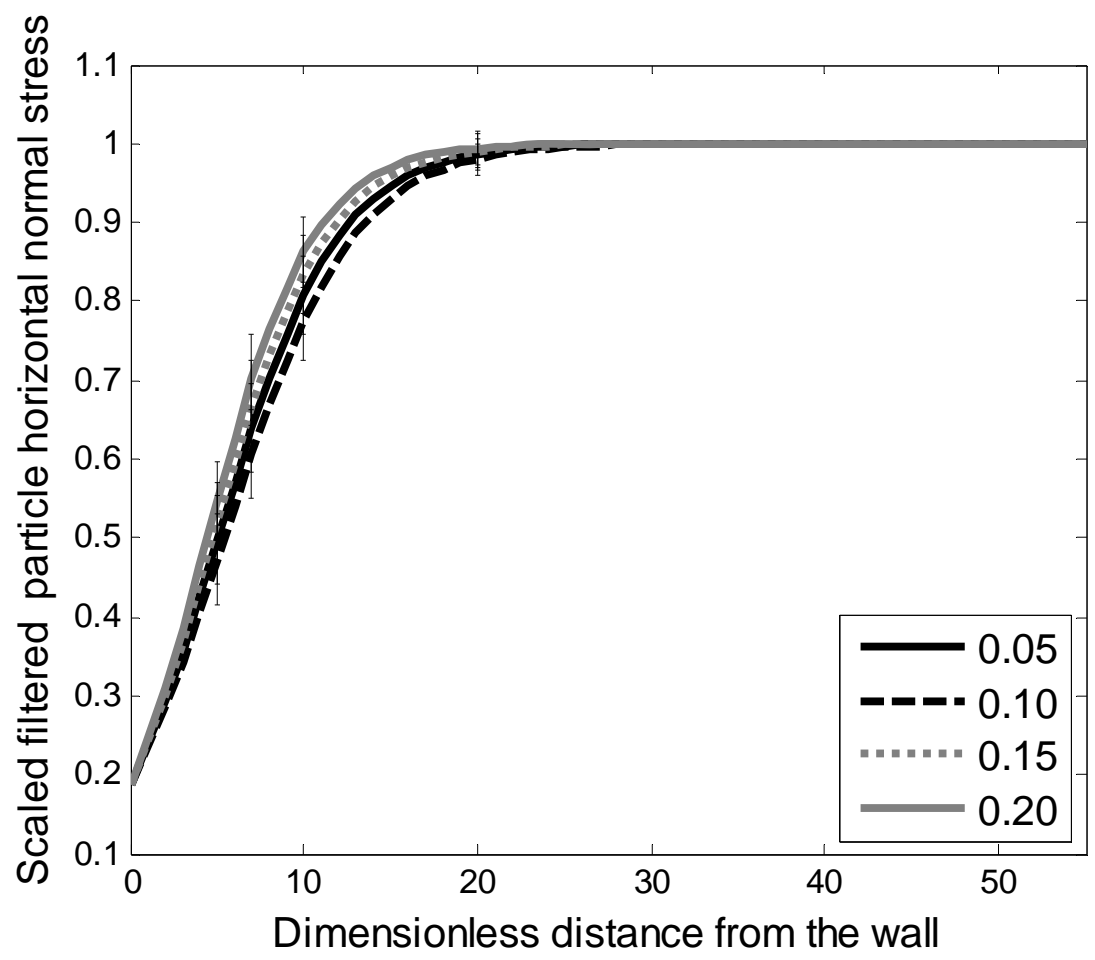


c)

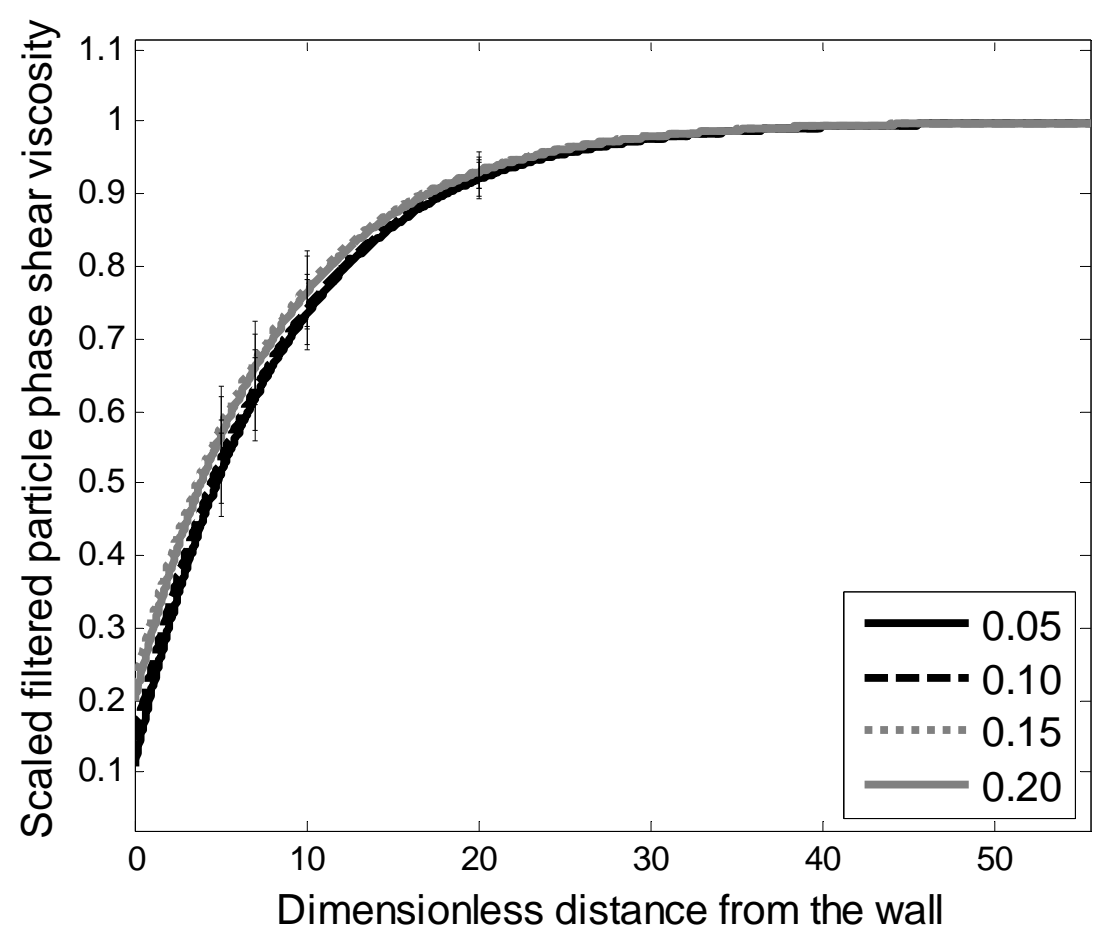

Figure 3: (a) The filtered drag coefficient, (b) the filtered horizontal particle phase normal stress, and (c) the filtered particle phase viscosity extracted from kinetic theory model based simulations in a channel shown in Figure 1. Error bars at four locations are also included. Results are shown for four different particle phase volume fractions inside the filtered region, illustrating that the wall correction is essentially independent of particle volume fraction. Grid length: 0.514 $d u$. Filter length: $2.056 \mathrm{du}$. The filtered drag coefficient is scaled with that extracted from the core region. The results are shown for one-half of the channel width and the distance is measured from the wall. Channel width and height are 102.80 and $1028 \mathrm{du}$, respectively. The dimensionless inlet gas and particle phase superficial velocities are 4.259 and 0.109 , respectively. The inlet particle phase volume fraction is $0.07 . \varphi: 0.6$. The remaining physical conditions corresponding to this simulation are listed in Table 1. 
a)

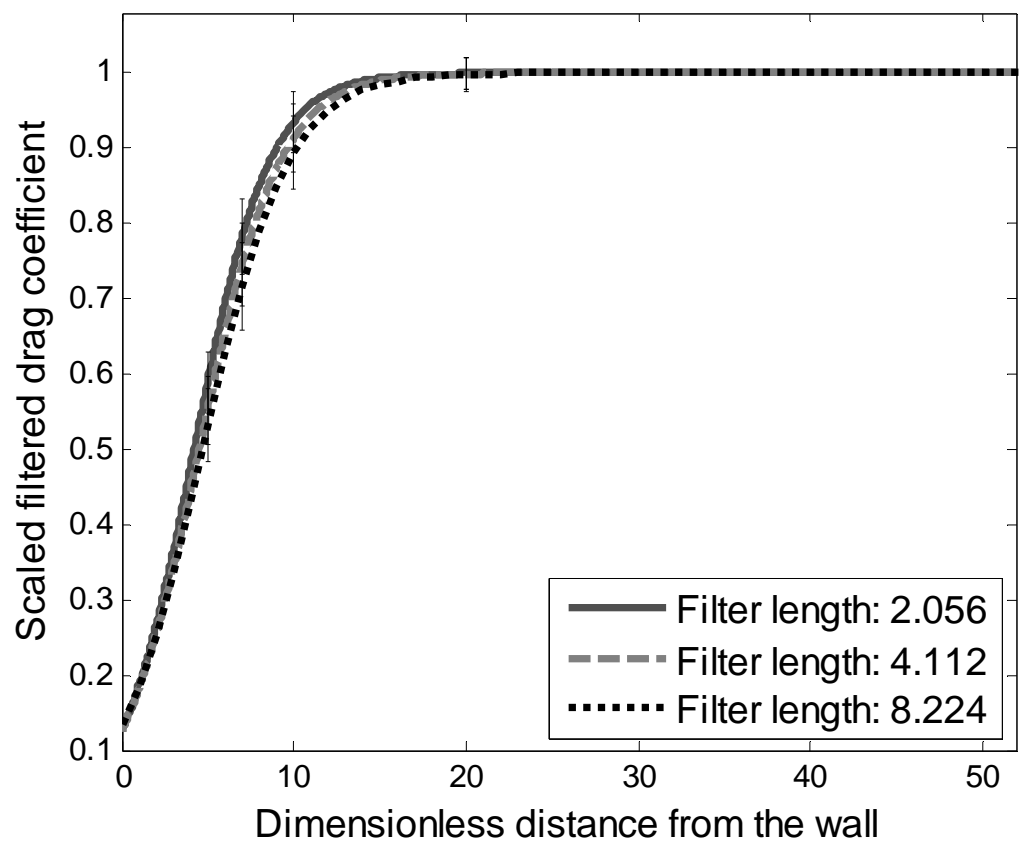

b)

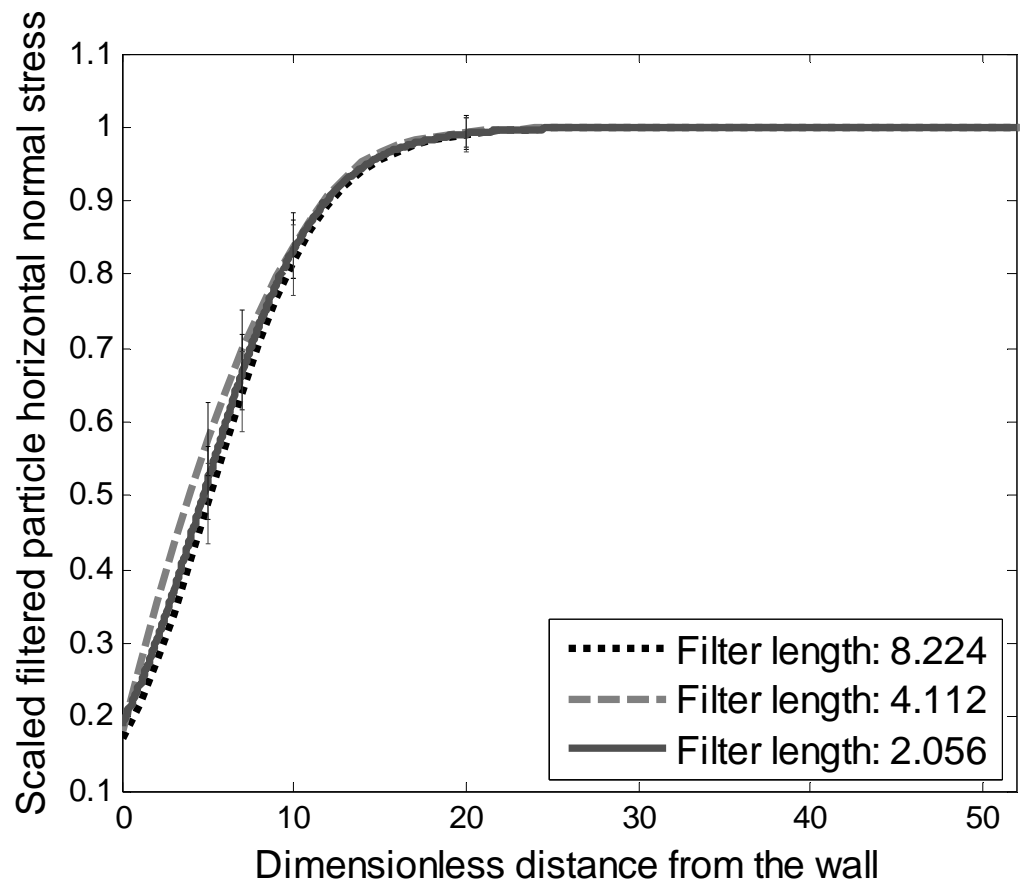


c)

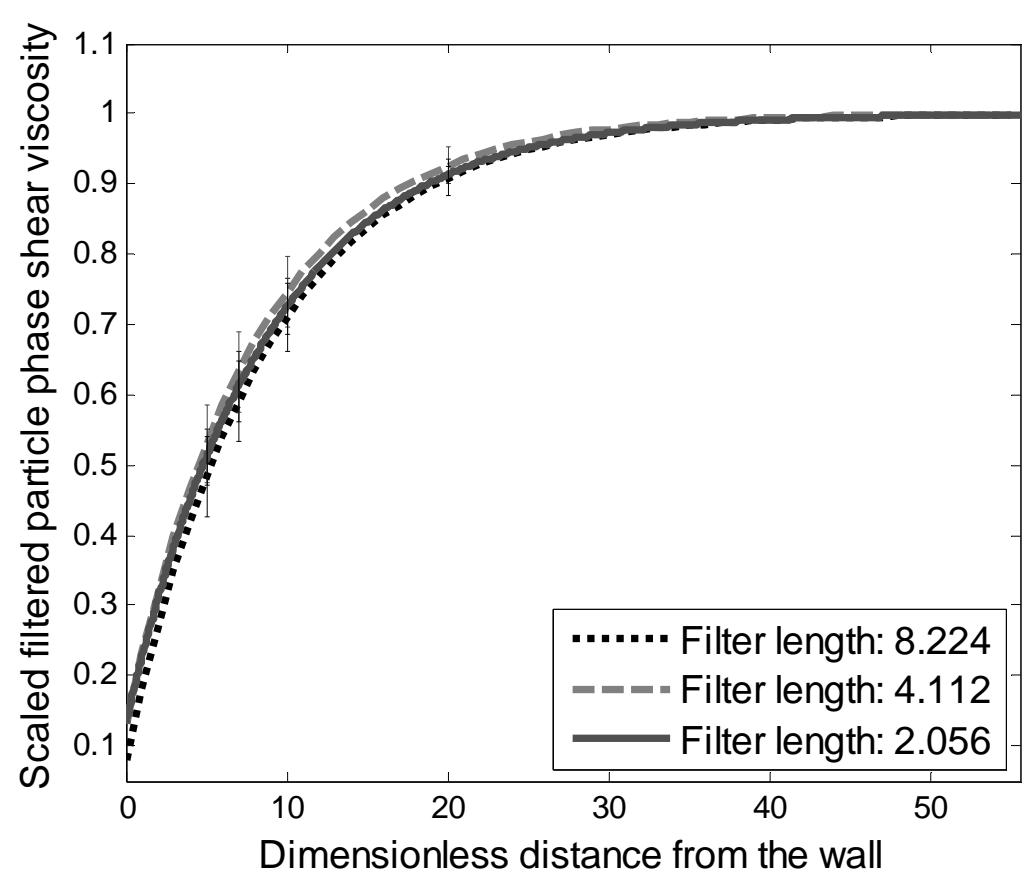

Figure 4: (a) Filtered drag coefficient, (b) filtered horizontal particle phase normal stress, and (c) filtered particle phase viscosity as functions of distance from the wall, extracted from kinetic theory model based simulations in the channel shown in Figure 1 for three filter lengths. Error bars at four locations are also included. Grid length: $0.514 d u$. The filtered drag coefficient is scaled with that extracted from the core region. The results are shown for one-half of the channel width and the distance is measured from the wall. See caption for Figure 3 for additional details. 
a)

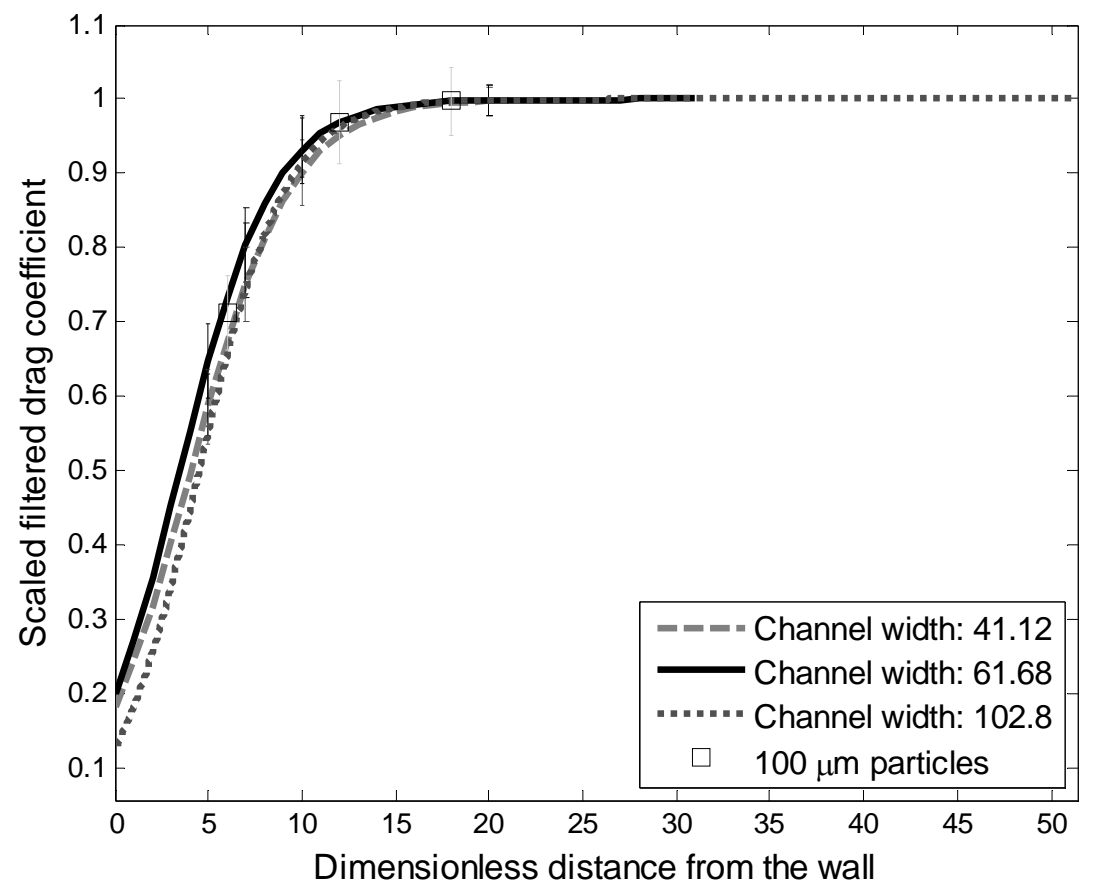

b)

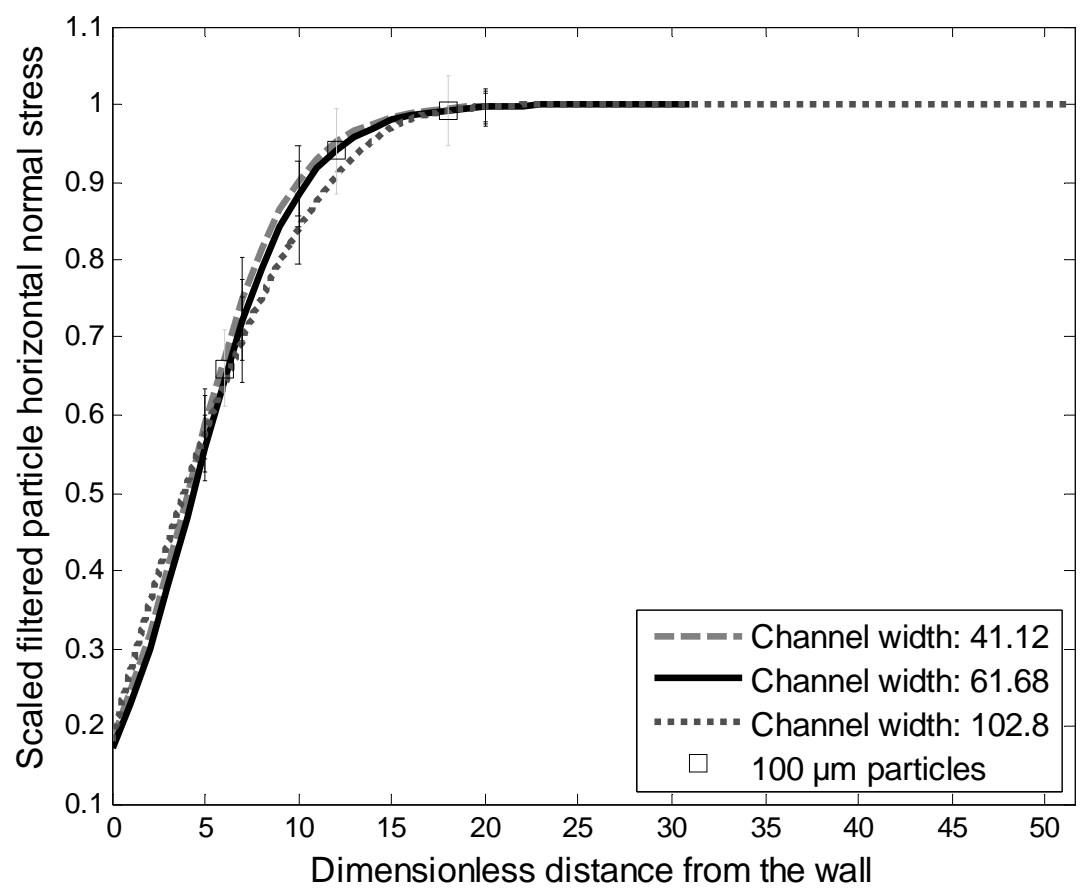


c)

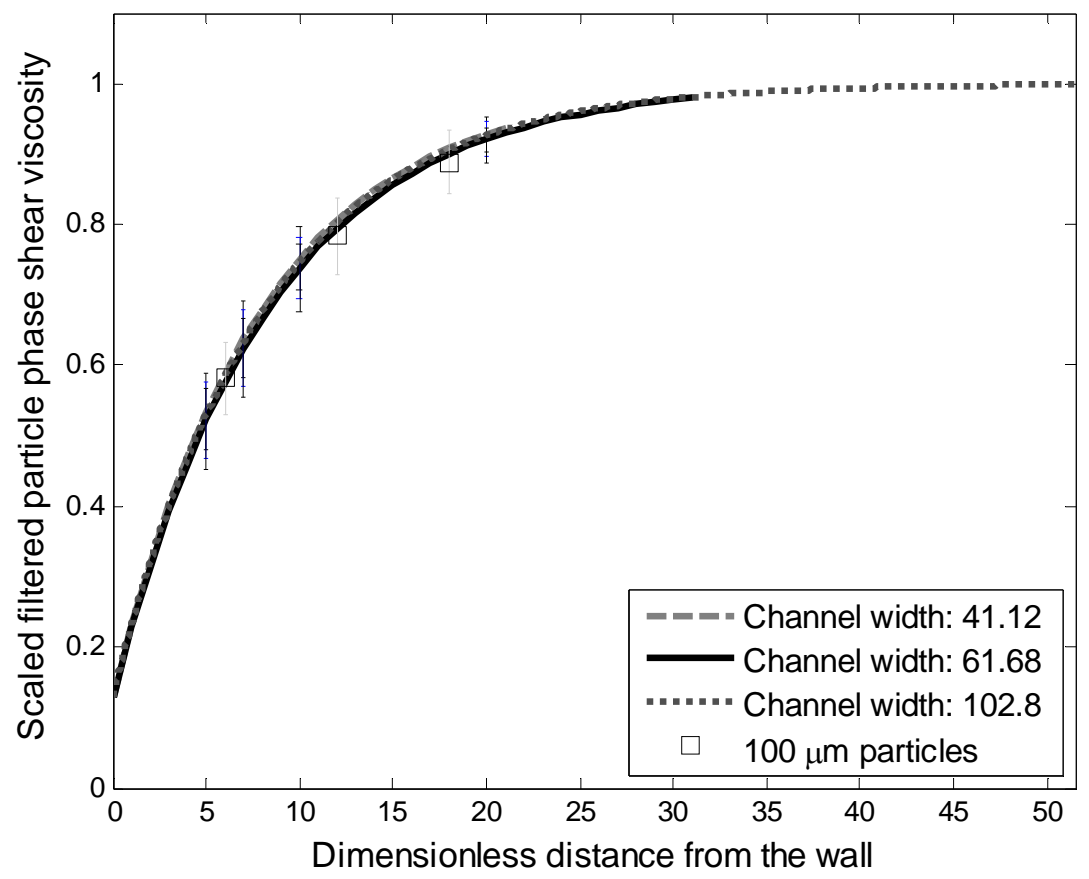

Figure 5: (a) Filtered drag coefficient, (b) filtered horizontal particle phase normal stress, and (c) filtered particle phase viscosity as functions of distance from the wall, extracted from kinetic theory model based simulations in the channel shown in Figure 1 for three different channel widths. Error bars at four locations are also included. Filter length: $2.056 \mathrm{du}$. Grid length: 0.514 $d u$. All filtered quantities were scaled with the corresponding quantities extracted from the core region (which were found to be the same for all three channel widths). See caption for Figure 3 for additional details. The figure also includes three data points (with error bars) from simulations with $100 \mu \mathrm{m}$ diameter particles. 
a)

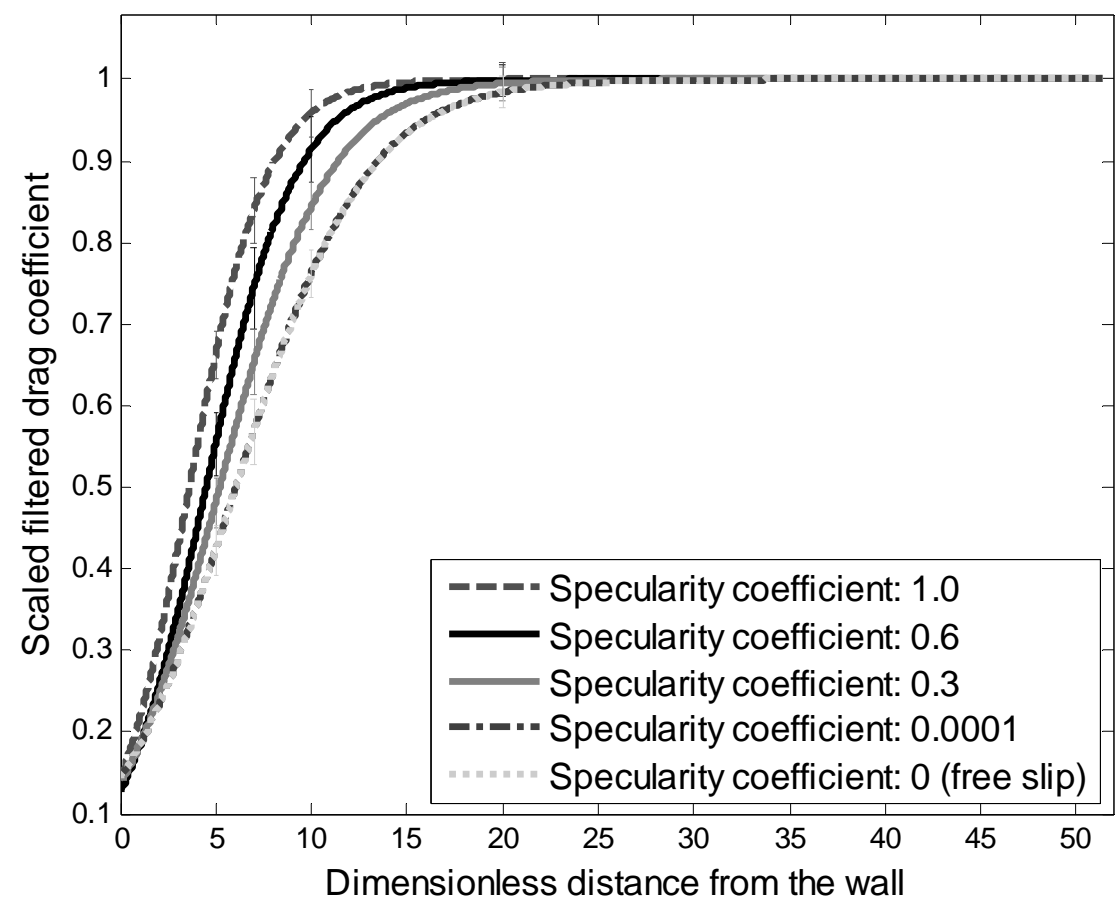

b)

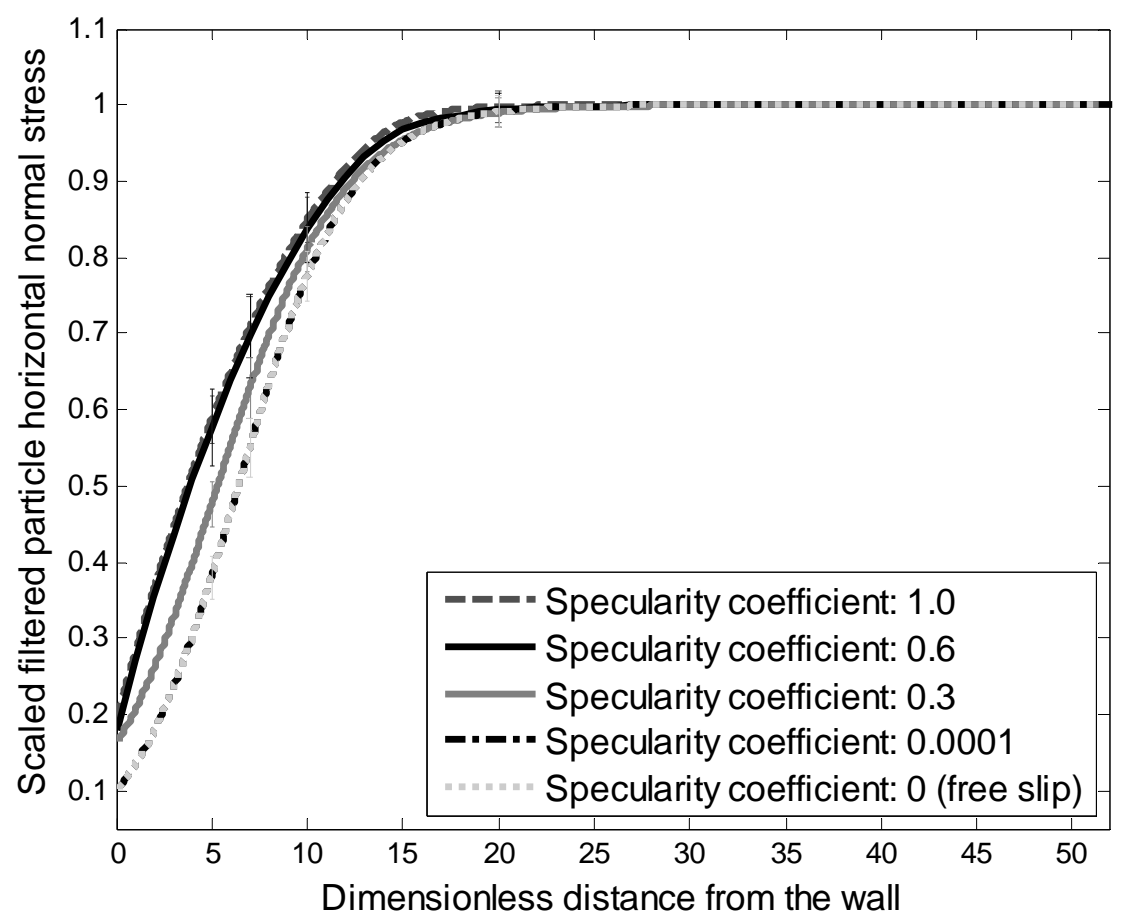


c)

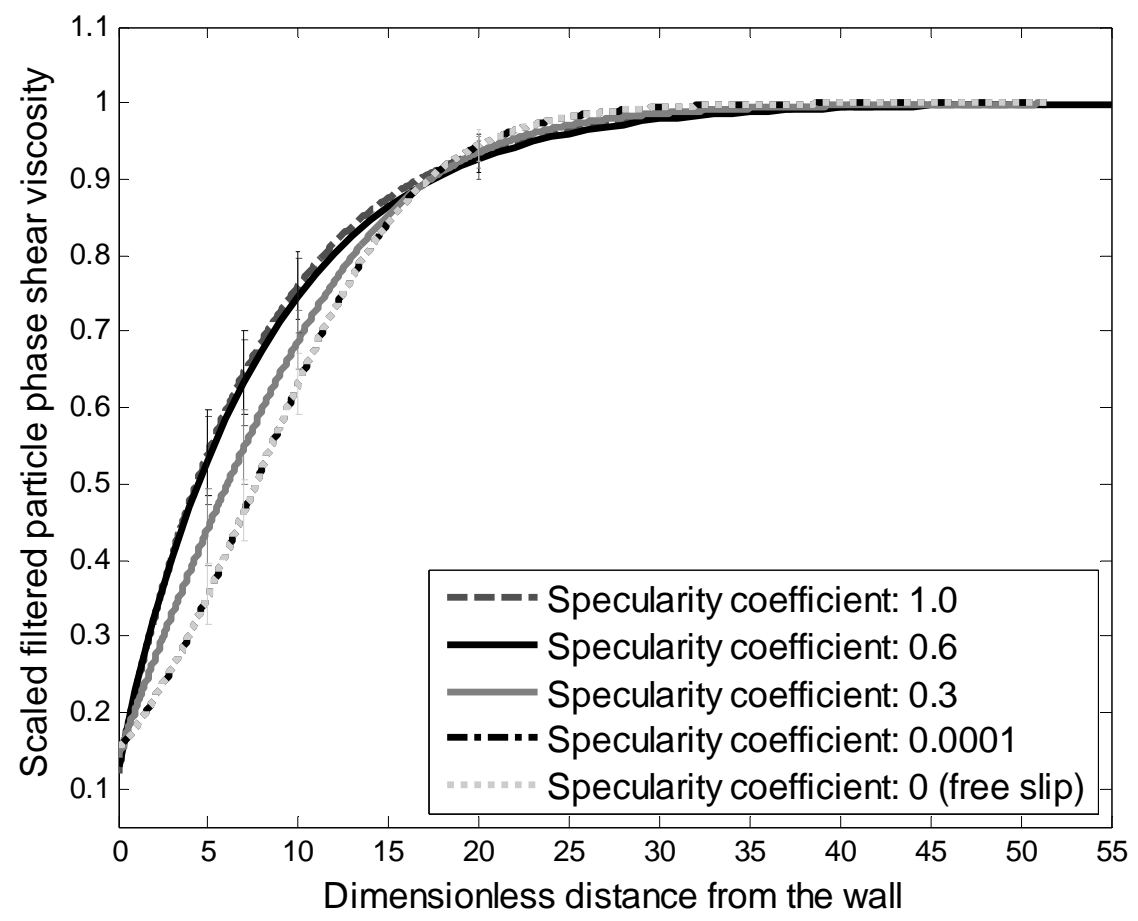

Figure 6: (a) Filtered drag coefficient, (b) filtered horizontal particle phase normal stress, and (c) filtered particle phase viscosity as functions of distance from the wall, extracted from kinetic theory model based simulations in the channel shown in Figure 1 for specularity coefficients of 0, 0.0001, 0.3, 0.6 and 1. Error bars at four locations are also included. Filter length: $4.112 \mathrm{du}$. Grid length: $0.514 \mathrm{du}$. Channel height: $1028 \mathrm{du}$. All filtered quantities were scaled with the corresponding quantities extracted from the core region. See caption for Figure 3 for additional details. 
(a)

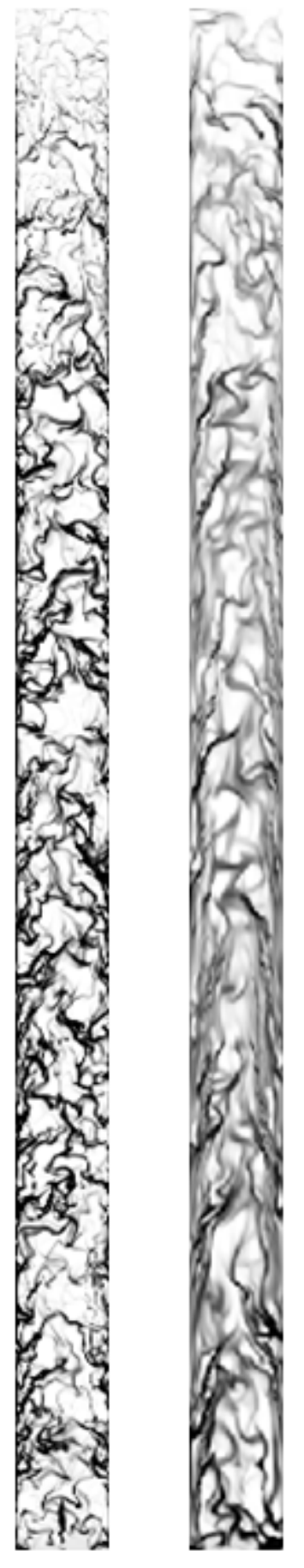

(c)

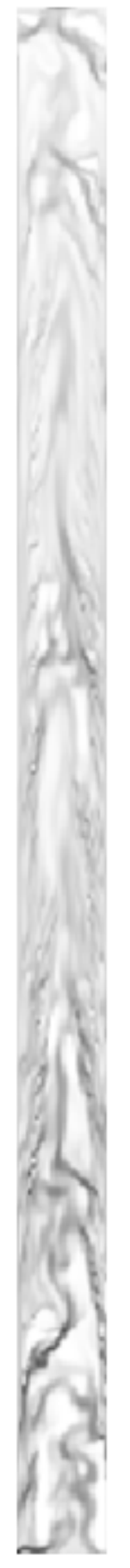

(d)

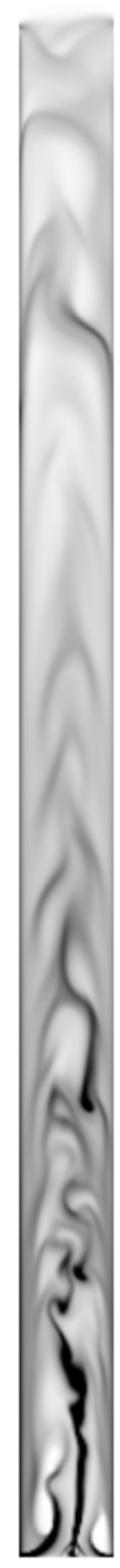

(e)

(f)

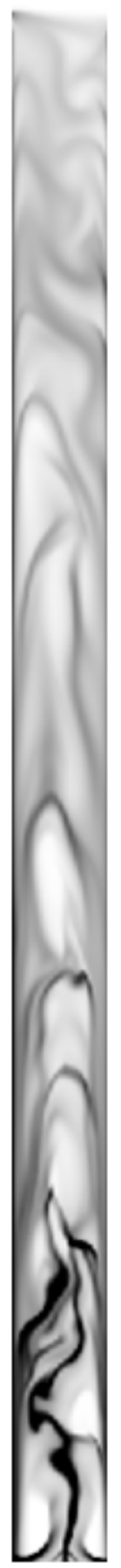

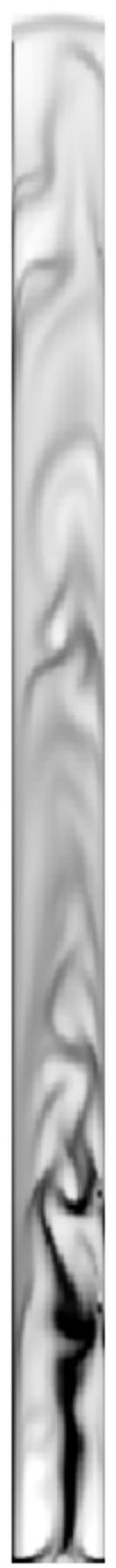


Figure 7: Snapshots of the particle phase volume fraction field extracted from the 2D simulation of (a) the kinetic theory model equations with $0.514 d u$ grids, (b) the kinetic theory model equations with $1.028 \mathrm{du}$ grids, (c) the kinetic theory model equations with $2.056 \mathrm{du}$ grids, (d) the filtered model equation with $0.514 d u$ grids, (e) the filtered model equation with $1.028 d u$ grids, (f) the filtered model equation with $2.056 \mathrm{du}$ grids. Wall corrections (extracted from kinetic theory simulations with $\varphi=0.6$ ) were included in the closures for the filtered model equations. Simulation conditions: The dimensionless inlet gas and particle phase superficial velocities are 4.259 and 0.109 , respectively. The physical conditions corresponding to this simulation are listed in Table 1. The inlet particle phase volume fraction is 0.07 . Free slip BC was imposed for both phases at all walls for simulations of the filtered models, whereas in the kinetic theory-based simulations, partial slip BC for the particle phase $(\varphi=0.6)$ and free slip BC for the gas phase were used. Channel width and height are 61.68 and $1028 \mathrm{du}$, respectively. The gray scale axis ranges from $\phi_{s}=0.00$ (white) to $\phi_{s}=0.45$ (black). 


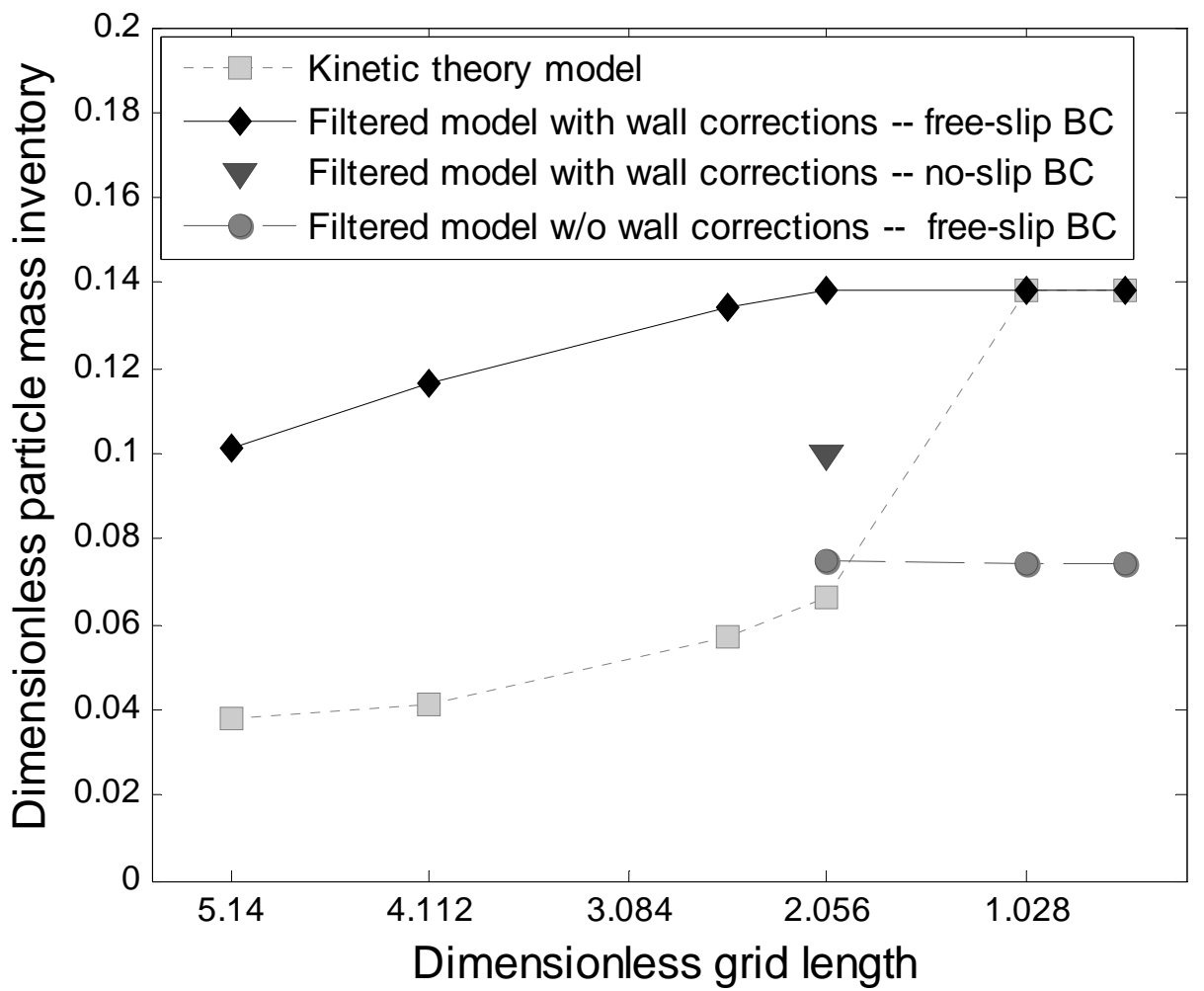

Figure 8: The variation of the particle mass inventory with grid resolution in the channel, determined from simulations of the kinetic theory based model and the filtered model (corresponding to a filter length of $4.112 \mathrm{du}$ ) with and without wall corrections. The particle mass inventory was scaled by the particle density times the volume of the bed. Note that the resolution increases from left to right. One data point (for a grid length of $2.056 \mathrm{du}$ ) obtained from the filtered model simulations with wall corrections and no slip BC for both phases was also included. See caption of Figure 7 for the simulation conditions. 
a)

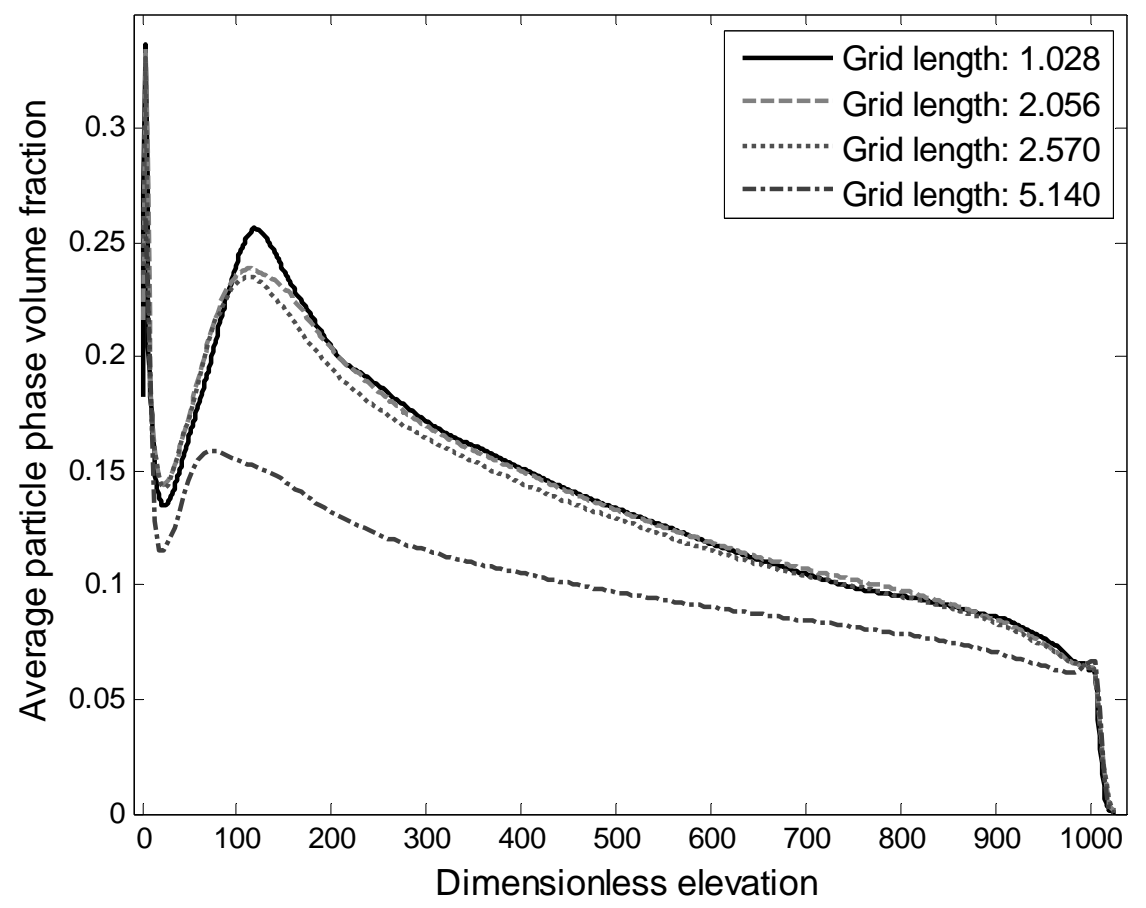

b)

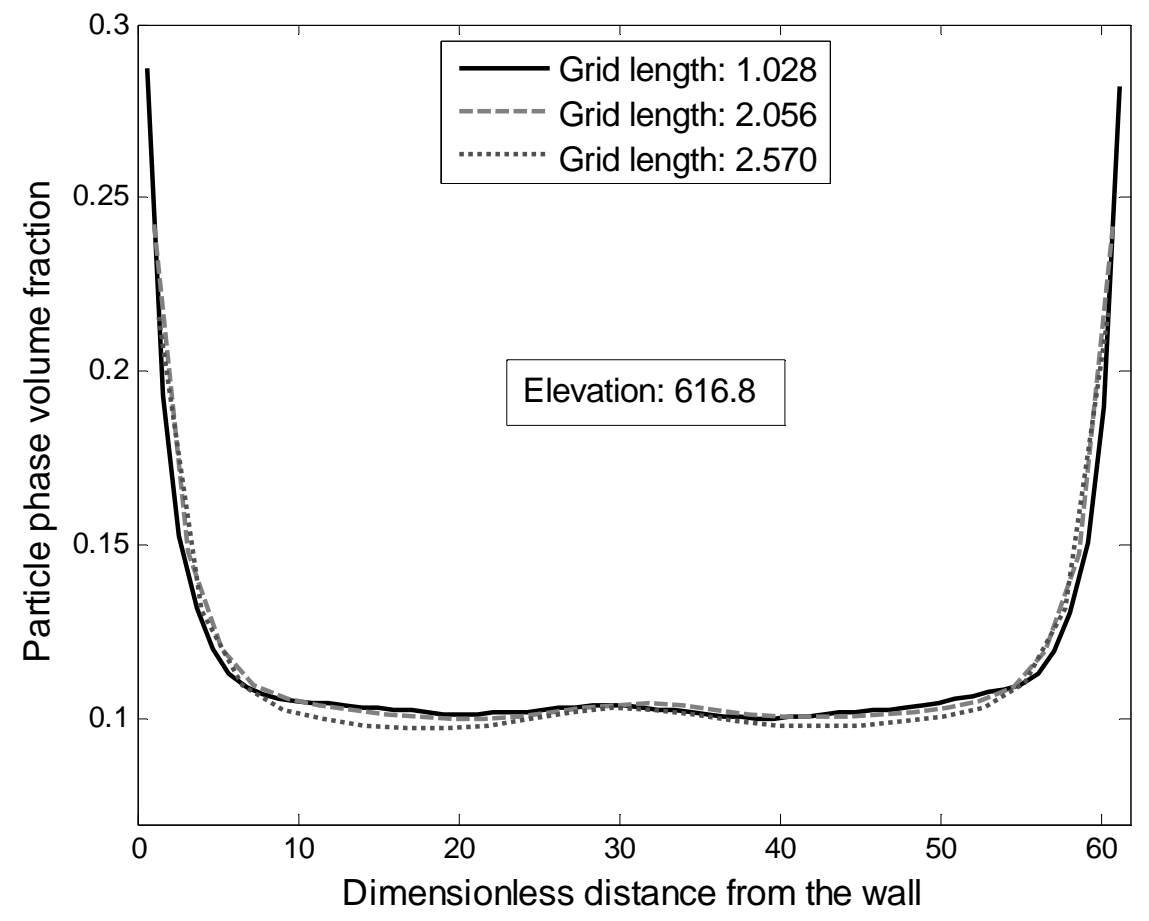


c)

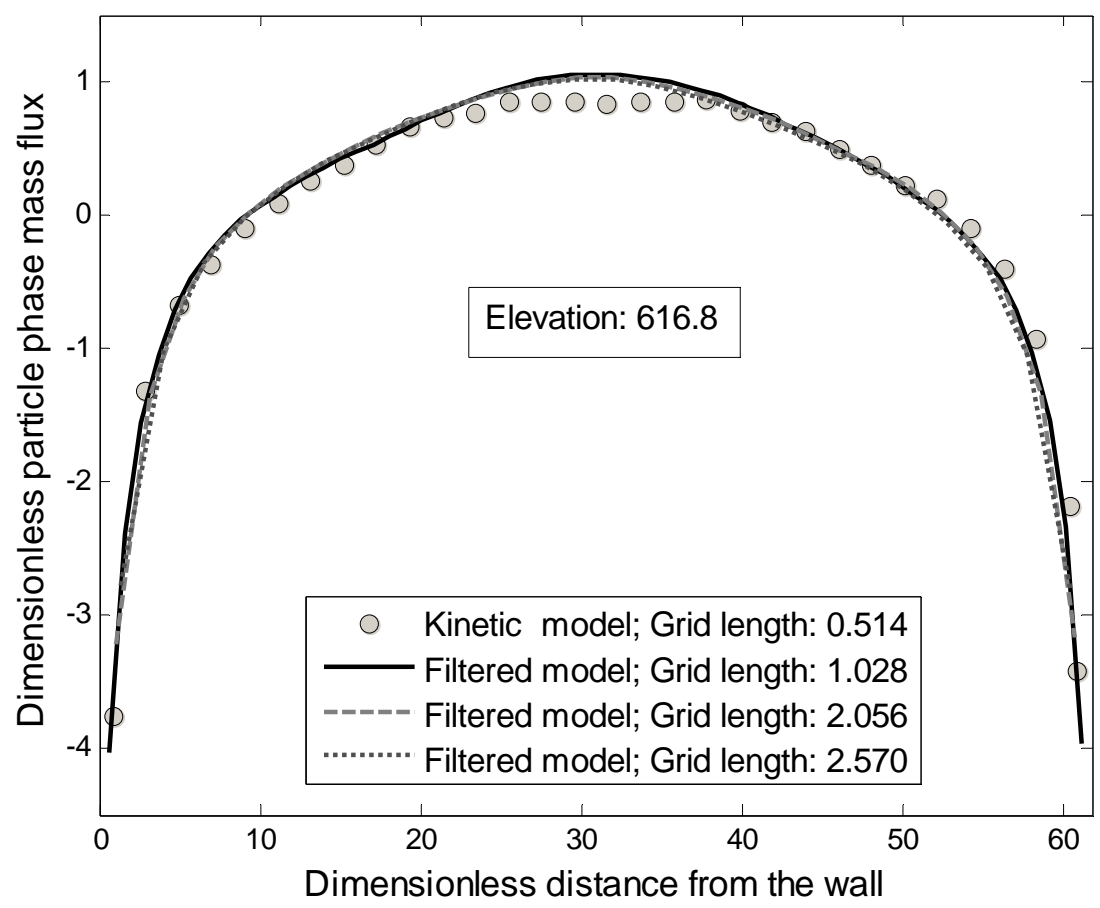

d)

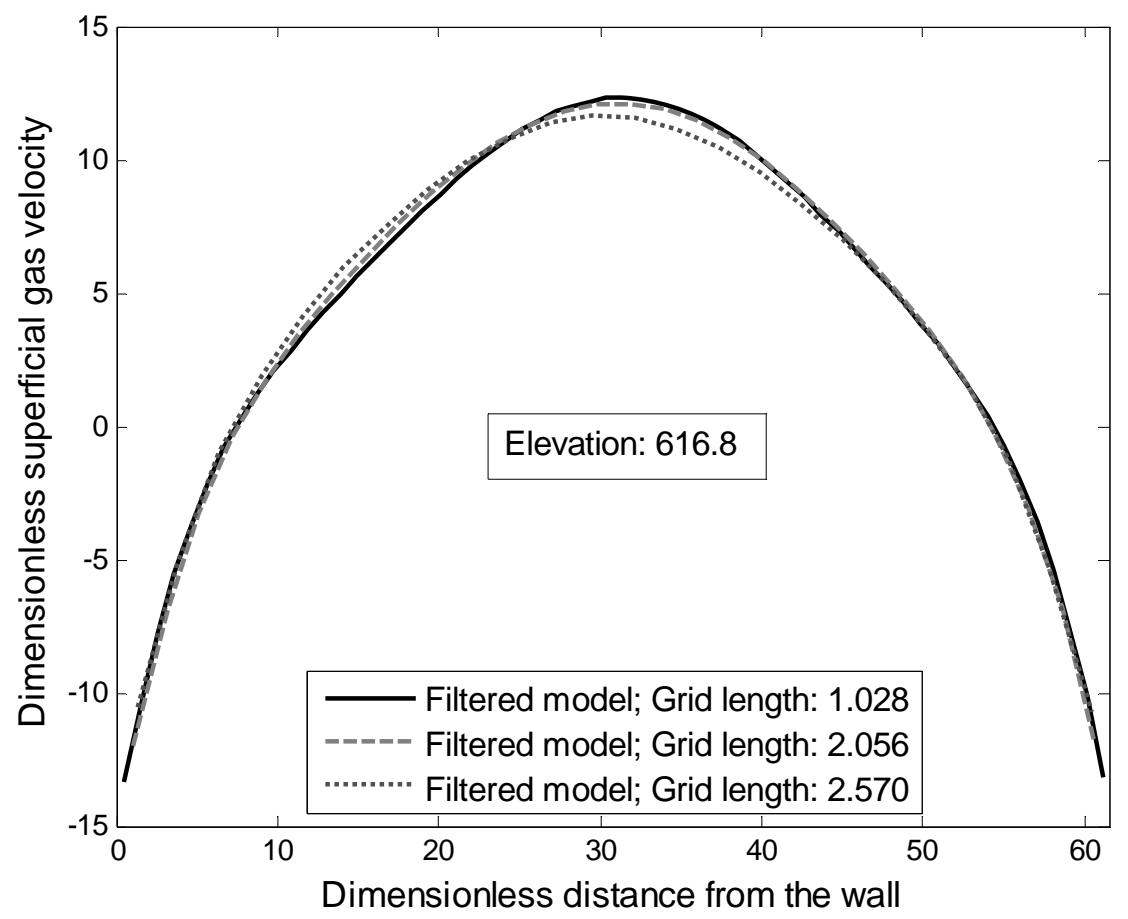


Figure 9: (a) The variation of temporally and laterally averaged particle phase volume fraction with the elevation in the 2D channel. The variation of time-averaged (b) particle phase volume fraction, (c) axial particle phase mass flux, and (c) axial superficial gas velocity with the dimensionless distance from the wall at an elevation of $616.6 \mathrm{du}$. Results were obtained from simulations of a filtered model with a filter length of $4.112 d u$ (including wall corrections) and different grid lengths, shown in the figure legends. See caption of Figure 7 for the simulation conditions. The circles in Figure 9(b) were obtained by solving the kinetic theory based model using a grid length of $0.514 \mathrm{du}$. 
(a)

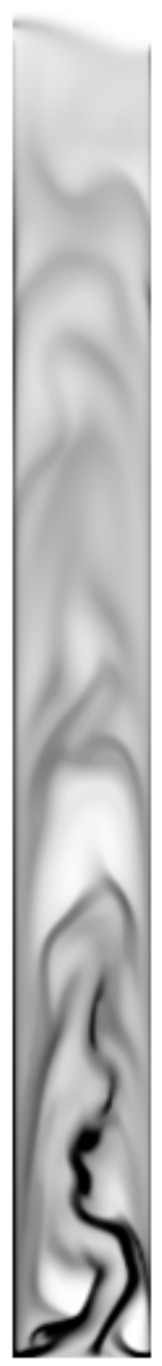

(b)

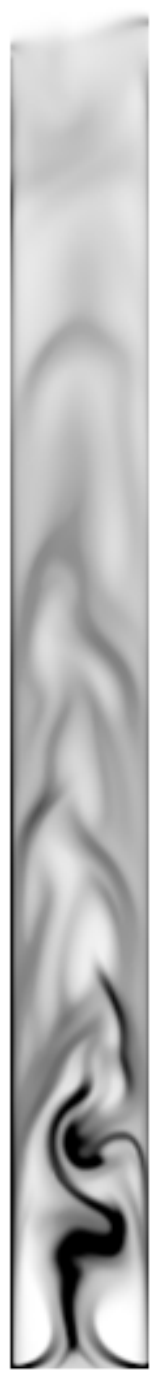

Figure 10: Snapshots of the particle phase volume fraction field extracted from the 2D simulation of the filtered model equations extracted with a filter length of (a) $4.112 d u$ and (b) $8.224 d u$. Simulation conditions: The dimensionless inlet gas and particle phase superficial velocities are 4.259 and 0.109 , respectively. The physical conditions corresponding to this simulation are listed in Table 1. The inlet particle phase volume fraction is 0.07. Grid length: $2.056 \mathrm{du}$. Free slip BC was used for both phases at all walls. Wall corrections (extracted from kinetic theory simulations with $\varphi=0.6$ ) are included. Channel width and height are 102.8 and $1028 d u$, respectively. The gray scale axis ranges from $\phi_{s}=0.00$ (white) to $\phi_{s}=0.45$ (black). 
a)

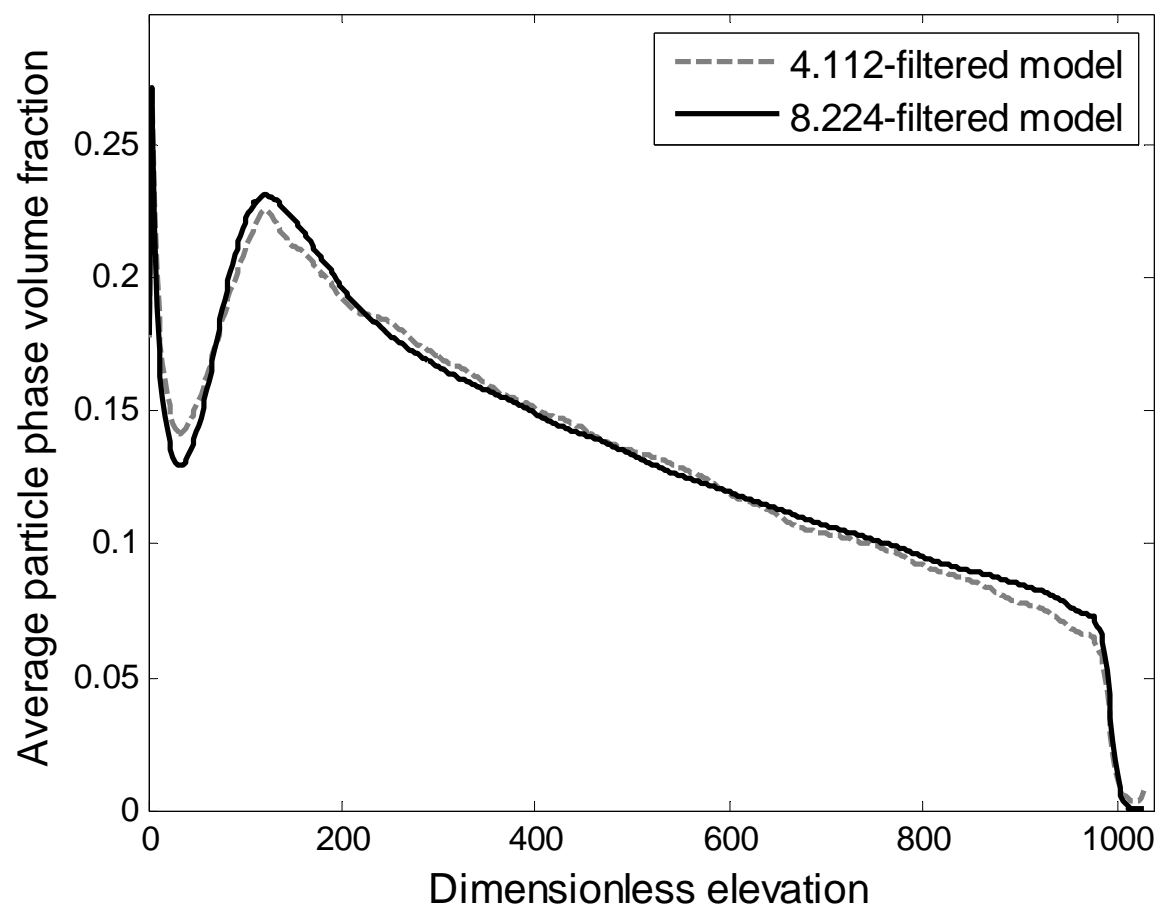

b)

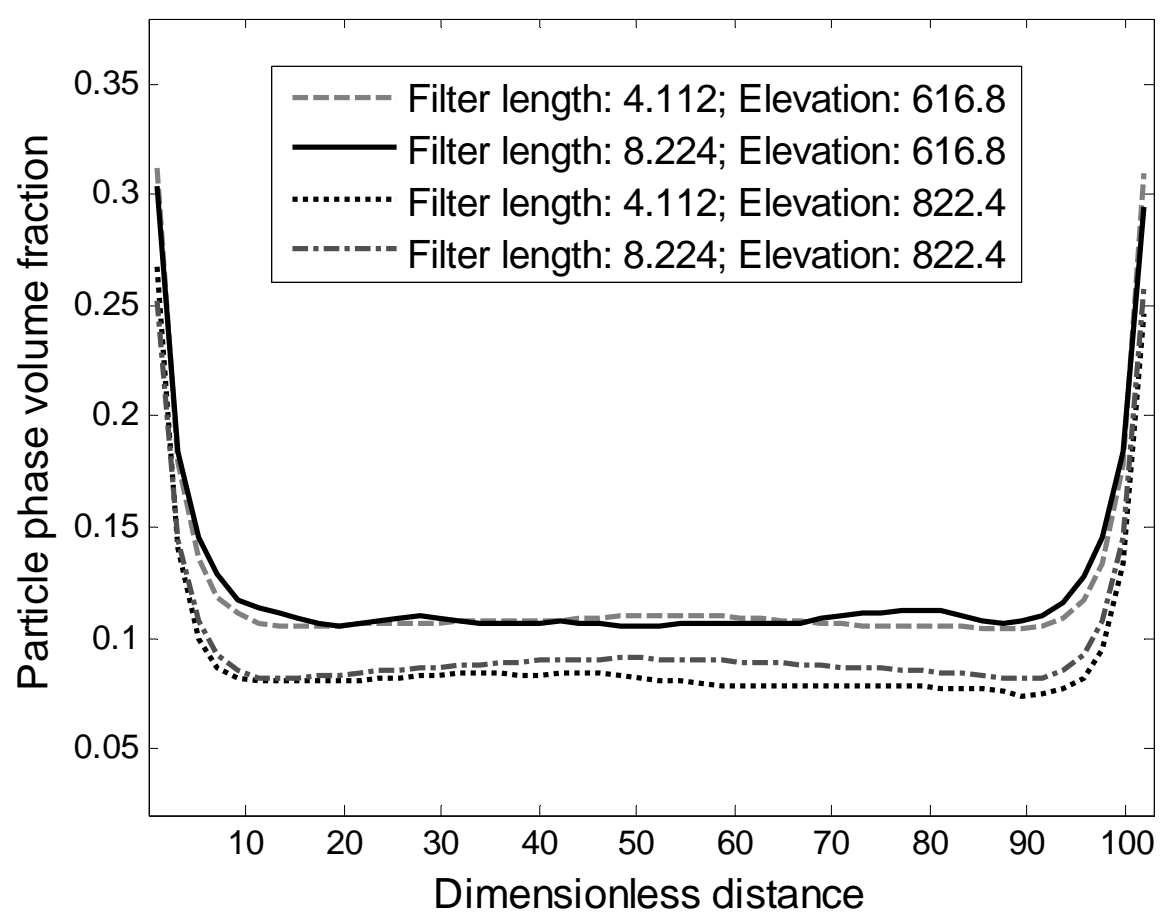


c)

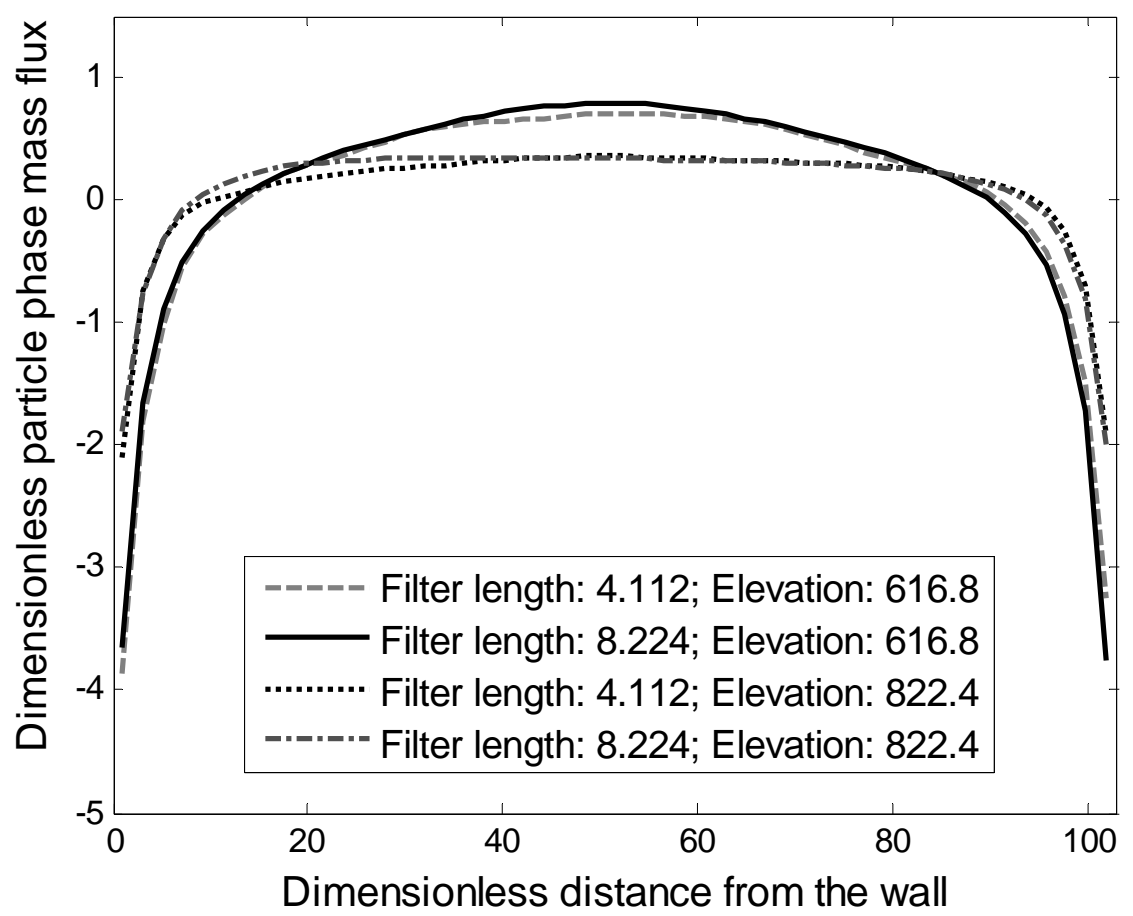

d)

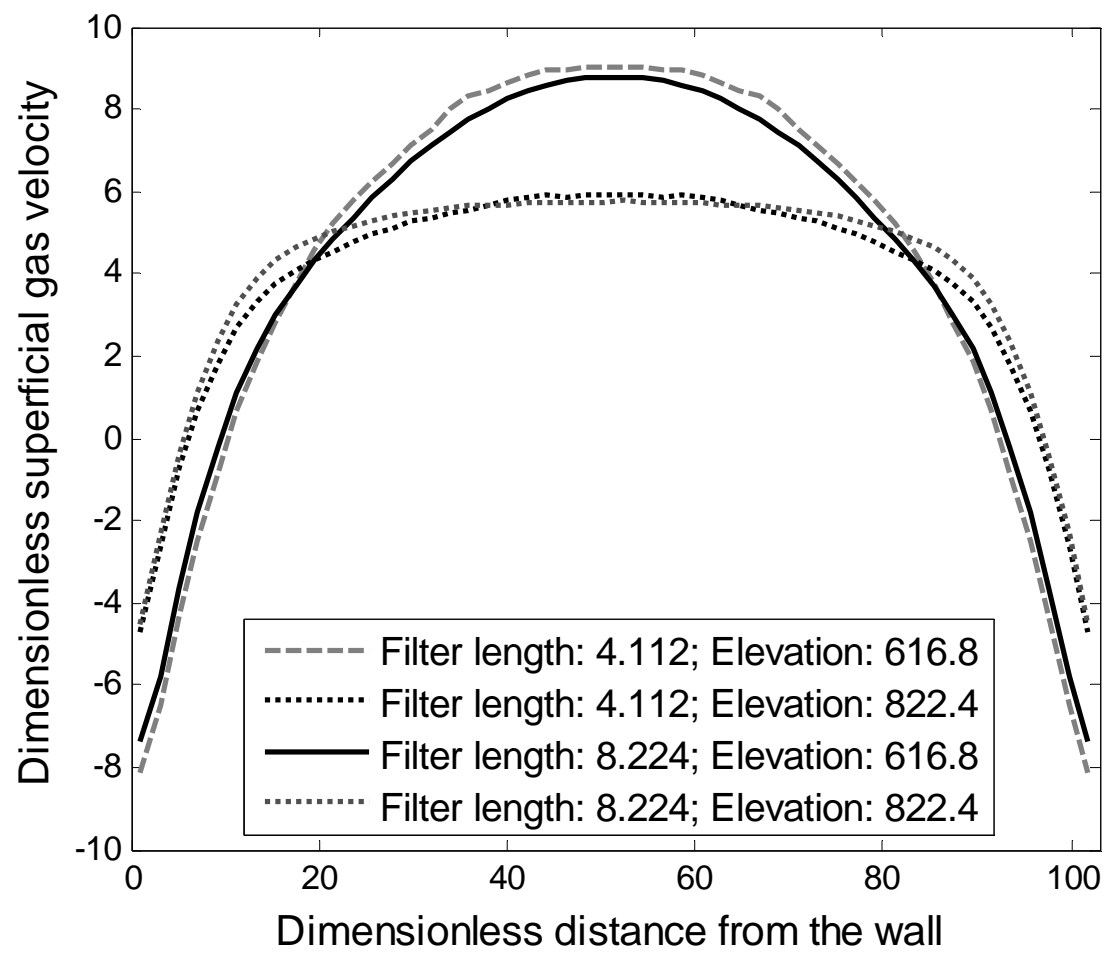


Figure 11: (a) The variation of temporally and laterally averaged particle volume fraction with the elevation in the $2 \mathrm{D}$ channel, determined from simulations of filtered models with filter lengths of 4.112- and $8.224 \mathrm{du}$. The variation of time-averaged (b) particle phase volume fraction, (c) axial particle phase mass flux, and (c) axial superficial gas velocity with the dimensionless distance from the wall at two elevations (616.8 and $822.4 \mathrm{du}$ ). Grid length: 2.056 $d u$. See caption of Figure 10 for the simulation conditions. 
(a)

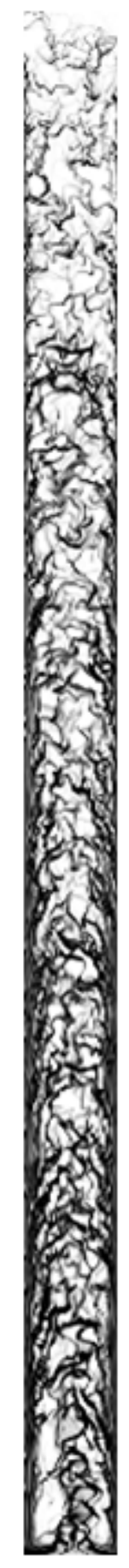

(b)

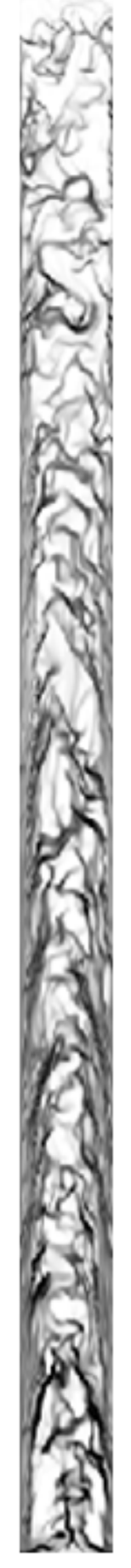

(c)

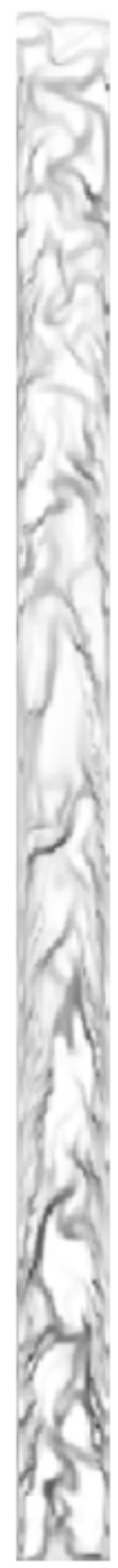

(d)

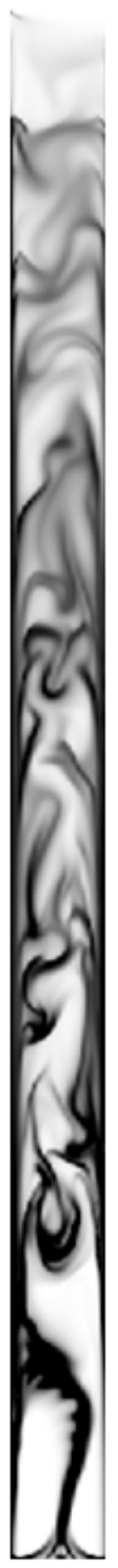


Figure 12: Snapshot of the particle phase volume fraction field extracted from the 2D simulation of (a) the kinetic theory model equations with $0.514 d u$ grids, (b) the kinetic theory model equations with $1.028 d u$ grids, (c) the kinetic theory model equations with $2.056 \mathrm{du}$ grids, (d) the filtered model equation with $1.028 d u$ grids. Wall corrections (extracted from kinetic theory simulations with $\varphi=0.0001$ ) were included in the closures for the filtered model equations. Simulation conditions: The dimensionless inlet gas and particle phase superficial velocities are 4.259 and 0.109 , respectively. The physical conditions corresponding to this simulation are listed in Table 1. The inlet particle phase volume fraction is 0.07 . Free slip BC was imposed for both phases at all walls for simulations of the filtered models, whereas in the kinetic theory-based simulations, partial slip BC for the particle phase $(\varphi=0.0001)$ and free slip $\mathrm{BC}$ for the gas phase were used. Channel width and height are 61.68 and $1028 d u$, respectively. The gray scale axis ranges from $\phi_{s}=0.00$ (white) to $\phi_{s}=0.45$ (black). 


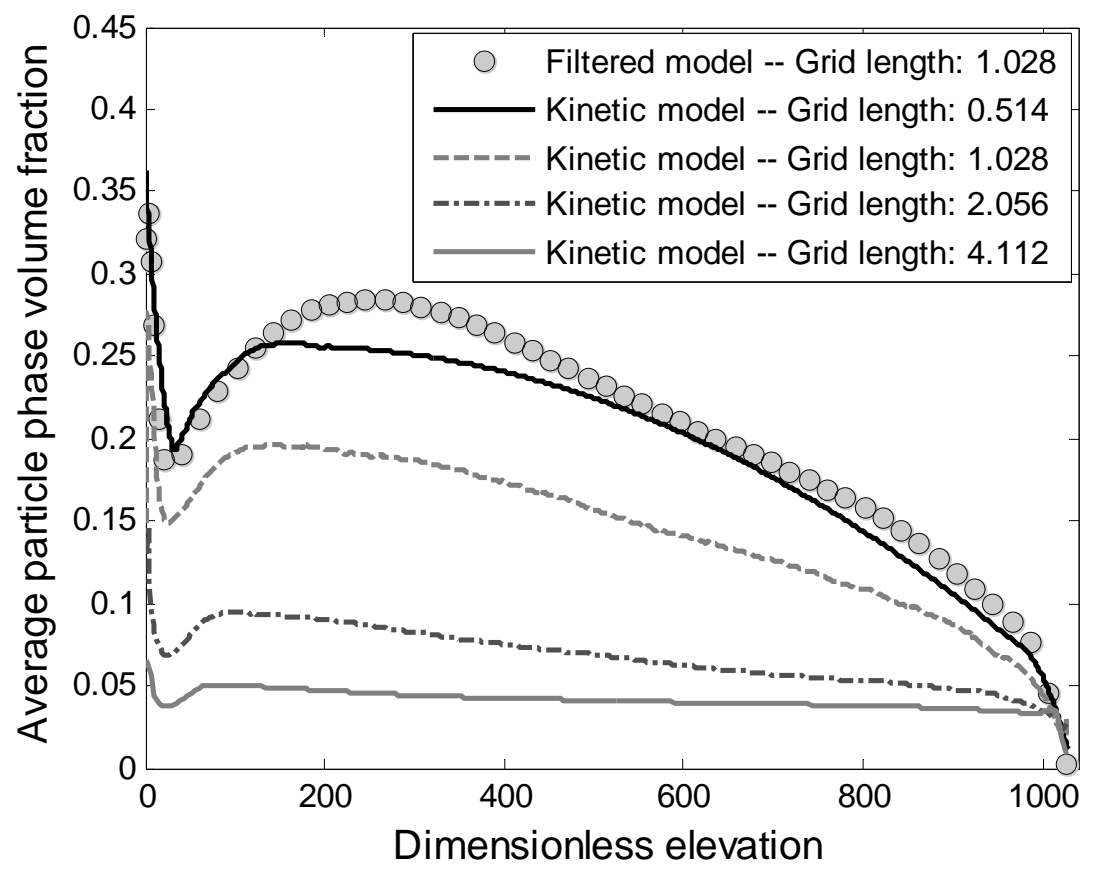

Figure 13: The variation of temporally and radially averaged particle volume fraction with the elevation in the 2D channel, whose snapshots are shown in Figure 12. Results were obtained from simulations of a filtered model with a filter length of $4.112 \mathrm{du}$ (including wall corrections) and different grid sizes. Results shown as lines were obtained from simulations of kinetic theory based model with different grid lengths, shown in the figure legend. The circles were obtained by solving the filtered model (filter length: $4.112 \mathrm{du}$ ) using a grid length of $1.028 \mathrm{du}$. See caption of Figure 12 for the simulation conditions. 
a)
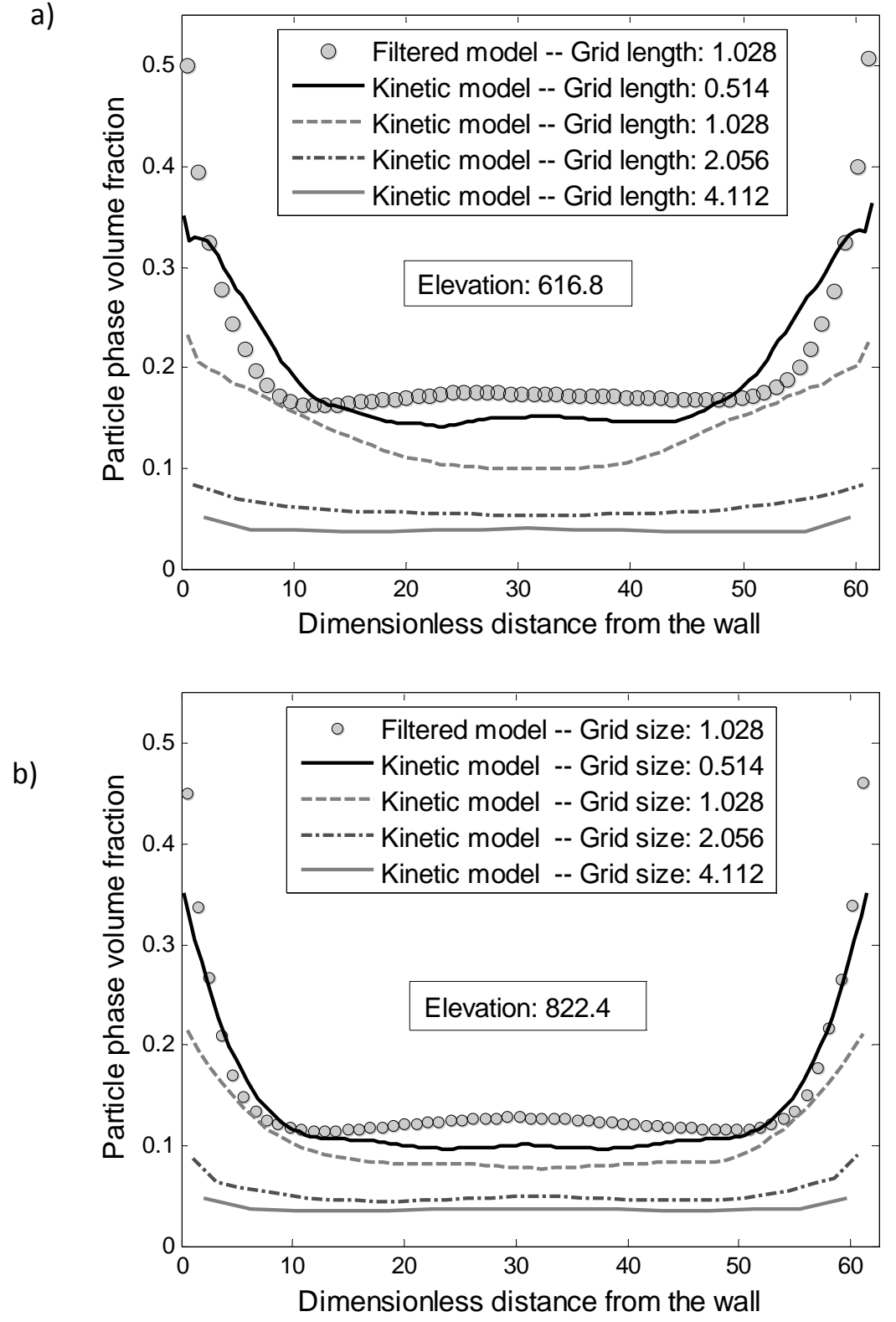

Figure 14: The variation of dimensionless time-averaged particle phase volume fraction with the dimensionless distance from the west (left) wall at two elevations (a) $616.8 d u$; (b) $822.4 d u$. Results shown as lines were obtained from simulations of kinetic theory based model with different grid lengths, shown in the figure legends. See caption of Figure 12 for the simulation conditions. The circles were obtained by solving the filtered model (filter length: $4.112 \mathrm{du}$ ) using a grid length of $1.028 \mathrm{du}$. 
a)

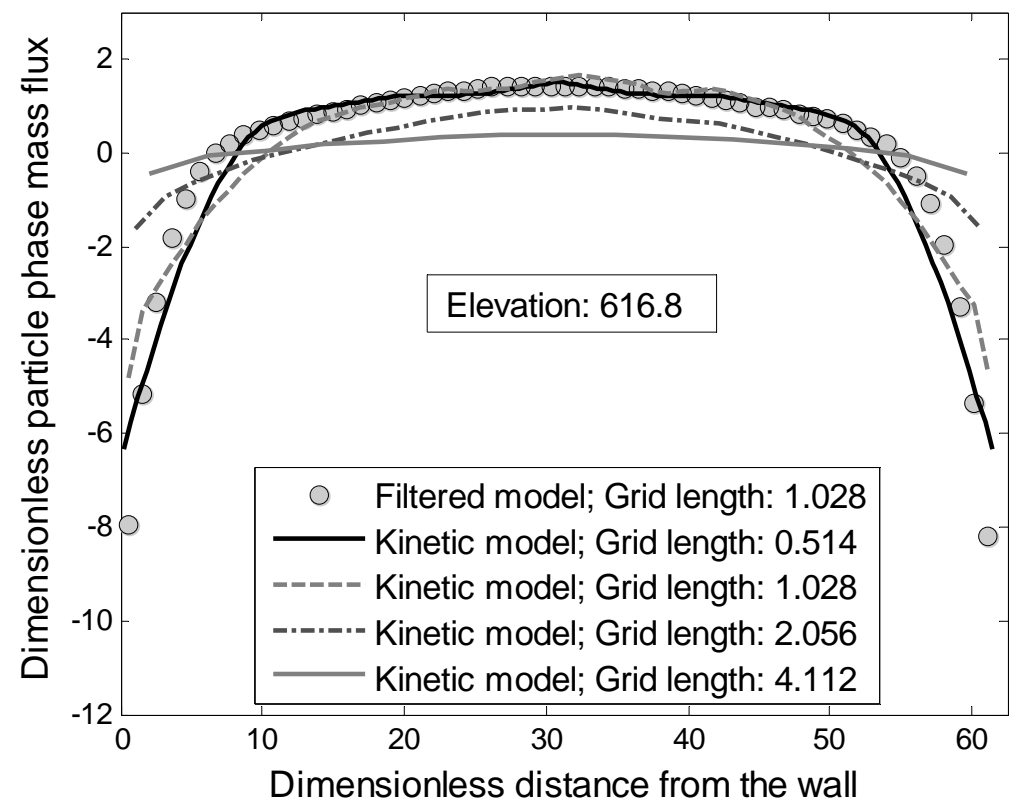

b)

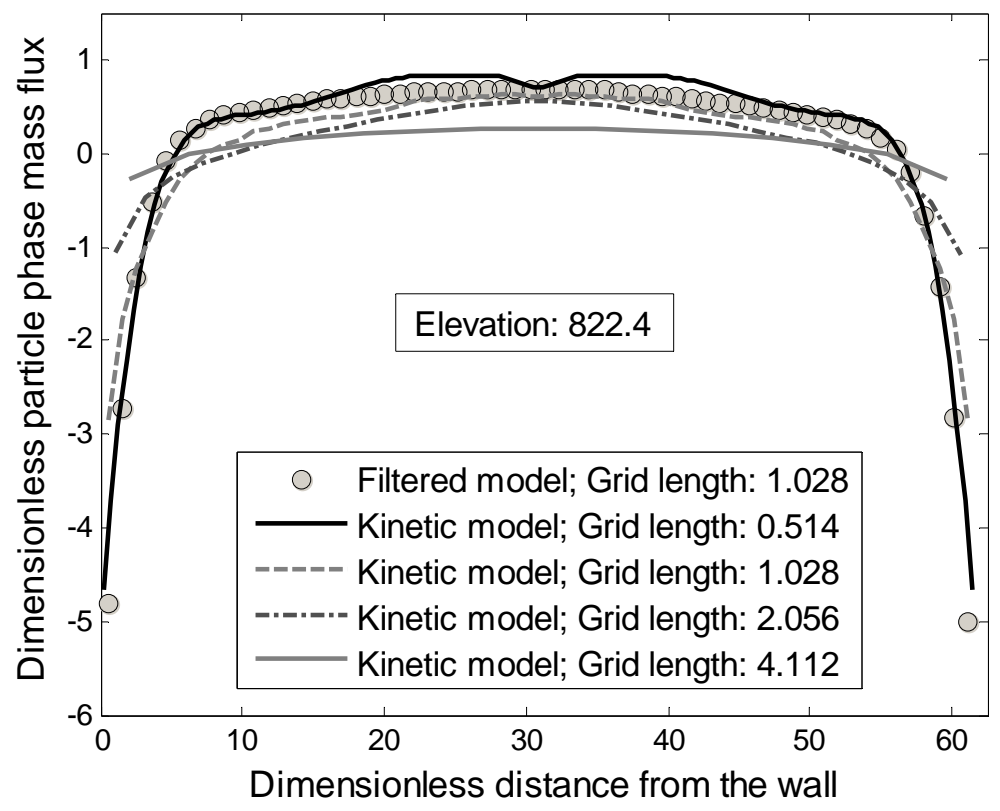

Figure 15: The variation of dimensionless time-averaged particle phase mass flux with the dimensionless distance from the west (left) wall at two elevations (a) $616.8 \mathrm{du}$; (b) $822.4 \mathrm{du}$. Results shown as lines were obtained from simulations of kinetic theory based model with different grid lengths, shown in the figure legends. See caption of Figure 12 for the simulation conditions. The circles were obtained by solving the filtered model (filter length: $4.112 \mathrm{du}$ ) using a grid length of $1.028 \mathrm{du}$. 
a)

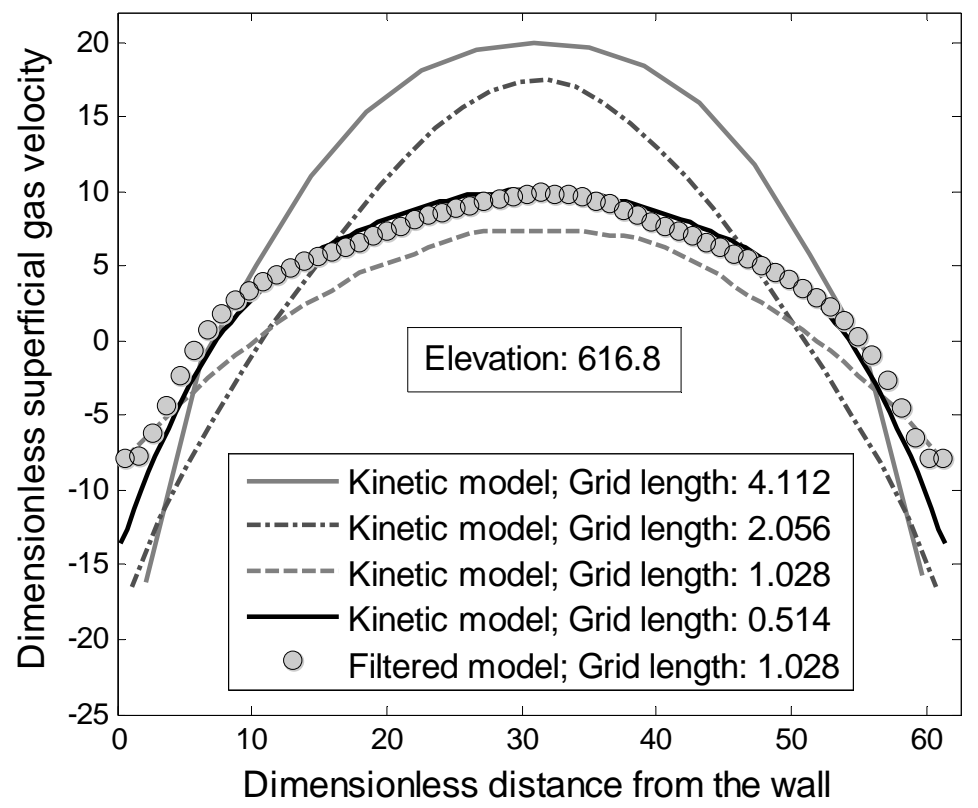

b)

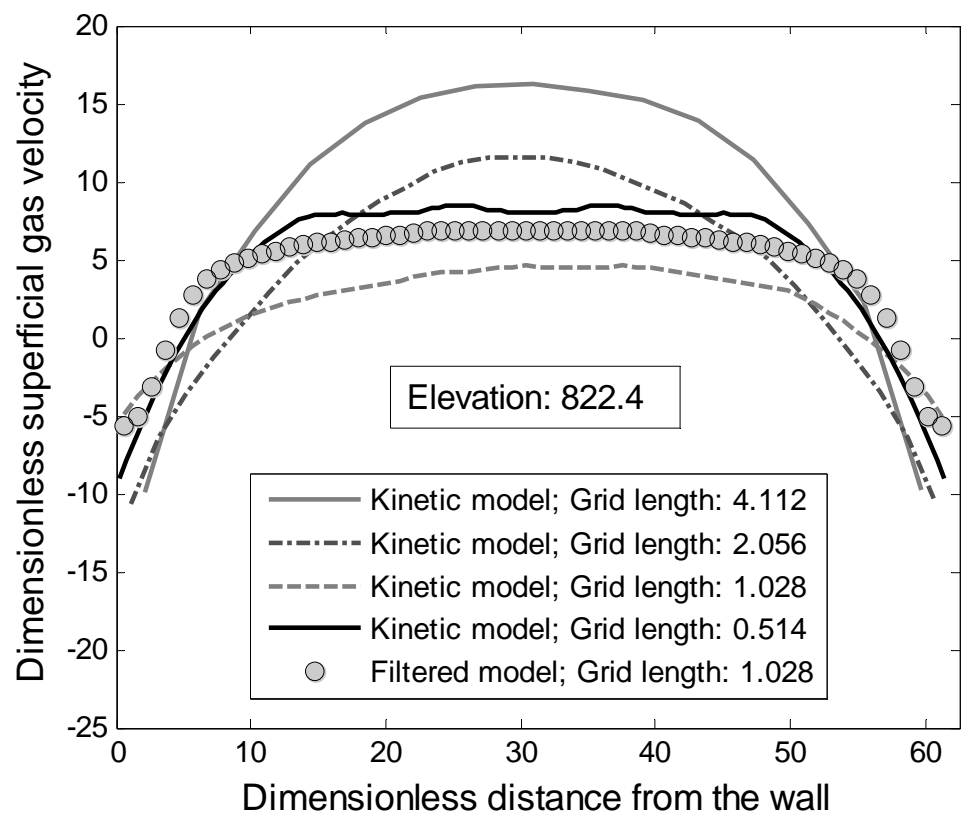

Figure 16: The variation of dimensionless time-averaged axial superficial gas velocity with the dimensionless distance from the west (left) wall at two elevations (a) $616.8 d u$; (b) $822.4 d u$. Results shown as lines were obtained from simulations of kinetic theory based model with different grid lengths, shown in the figure legends. See caption of Figure 12 for the simulation conditions. The circles were obtained by solving the filtered model (filter length: $4.112 \mathrm{du}$ ) using a grid length of $1.028 \mathrm{du}$. 


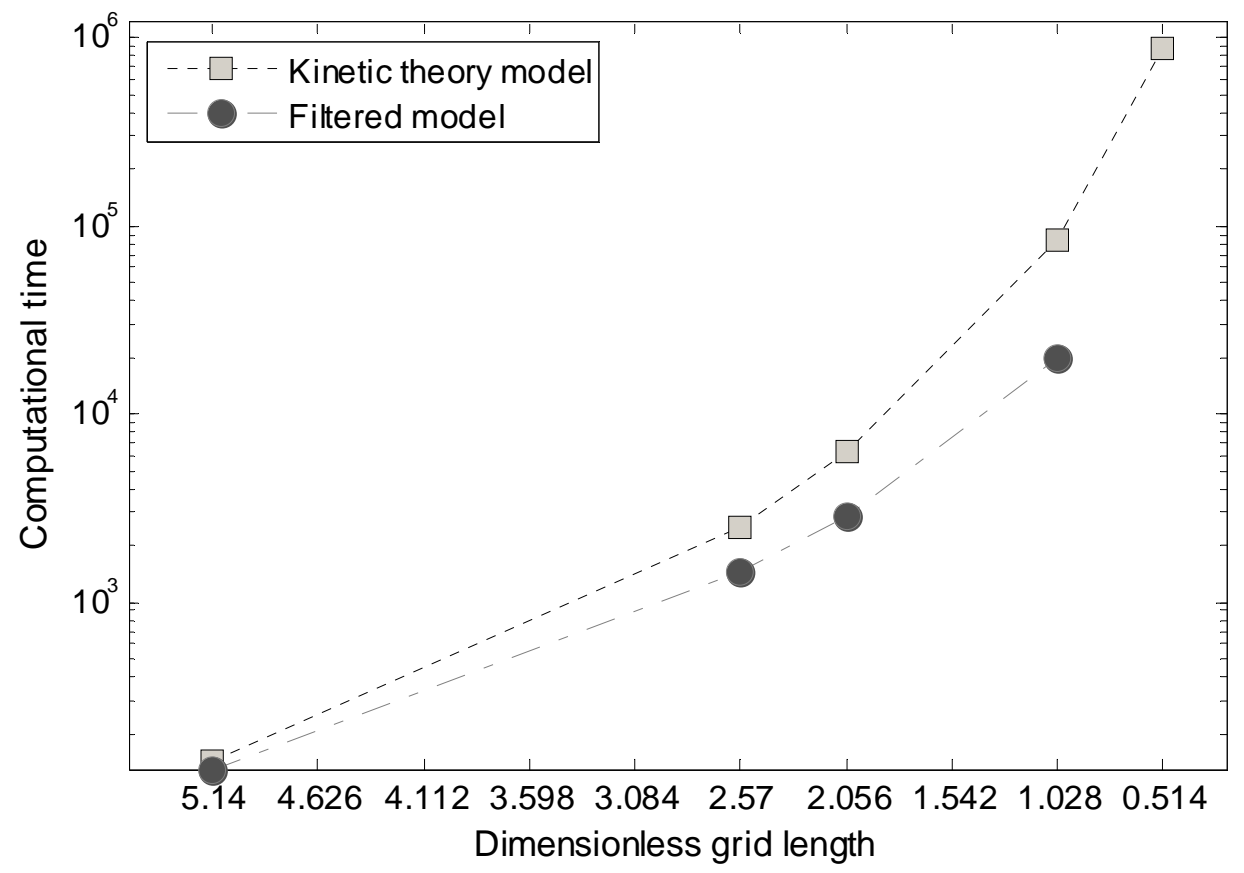

Figure 17: CPU times required to run one simulation second for the kinetic theory based twofluid model and a filtered model with a filter length of $4.112 d u$ at different grid resolutions. Note that the resolution increases from left to right. See caption of Figure 7 for the simulation conditions. The simulations were performed on Dual quad core Intel Xeon E5420 processors running at $2.50 \mathrm{GHz}$. 


\section{TABLES:}

Table 1: Sample physical properties of the gas and particles

\begin{tabular}{lll}
\hline$d_{p}$ & Particle diameter & $7.5 \times 10^{-6} \mathrm{~m}$ \\
$\rho_{\mathrm{s}}$ & Particle density & $1500 \mathrm{~kg} / \mathrm{m}^{3}$ \\
$\rho_{\mathrm{g}}$ & Gas density & $1.3 \mathrm{~kg} / \mathrm{m}^{3}$ \\
$\mu_{\mathrm{g}}$ & Gas viscosity & $1.8 \times 10^{-5} \mathrm{~kg} / \mathrm{m} \cdot \mathrm{s}$ \\
$e_{p}$ & Coefficient of restitution & 0.9 \\
$e_{w}$ & $e$ for particle-wall collisions & 0.9 \\
$\mathrm{~g}$ & Gravitational acceleration & $9.80665 \mathrm{~m} / \mathrm{s}^{2}$ \\
$\mathrm{v}_{\mathrm{t}}$ & Terminal settling velocity & $0.2184 \mathrm{~m} / \mathrm{s}$ \\
$\frac{\mathrm{v}_{\mathrm{t}}^{2}}{\mathrm{~g}}$ & Characteristic length & $0.00487 \mathrm{~m}$ \\
$\frac{\mathrm{v}_{\mathrm{t}}}{\mathrm{g}}$ & Characteristic time & \\
$\rho_{\mathrm{s}} \mathrm{v}_{\mathrm{t}}^{2}$ & Characteristic stress & $0.0223 \mathrm{~s}$ \\
$\rho_{\mathrm{s}} \mathrm{v}_{\mathrm{t}}$ & Characteristic mass flux & $71.55 \mathrm{~kg} / \mathrm{m} \cdot \mathrm{s}^{2}$ \\
\hline
\end{tabular}


Table 2: Wall corrections to the filtered drag coefficient, horizontal particle phase normal stress, and shear viscosity:

Dimensionless distance from the wall (see Figure 1): $\mathrm{x}_{d}=g \mathrm{x} / \mathrm{v}_{t}^{2}$

\section{Filtered drag coefficient:}

$\overline{\beta_{\text {filtered, scaled }}}=\frac{\overline{\beta_{\text {filtered }}}\left(\bar{\phi}_{s}, \mathrm{x}_{d}\right)}{\overline{\beta_{\text {filtered,core }}}\left(\bar{\phi}_{s}\right)}=\frac{1}{1+6.00 \exp \left(-a \mathrm{x}_{d}\right)}, \mathrm{x}_{d} \leq \frac{\text { channel width }}{2}$

$\overline{\beta_{\text {filtered,core }}}\left(\overline{\phi_{s}}\right)=\overline{\beta_{\text {filtered, periodic }}}\left(\overline{\phi_{s}}\right)$

where "a" is a function of the wall specularity coefficient $(\varphi)$ and is defined as:

$a(\varphi)=0.036 \varphi^{2}+0.162 \varphi+0.295$

\section{Filtered particle phase horizontal normal stress:}

For $\varphi=0.0$ (free slip):

$$
\overline{p_{s, \text { filtered }, \mathrm{xx}, \text { scaled }}}=\frac{\overline{p_{s, \text { filtered }, \mathrm{xx}}}\left(\overline{\phi_{s}}, \mathrm{x}_{d}\right)}{\overline{p_{s, \text { filtered } \mathrm{xx}, \text { core }}}\left(\overline{\phi_{s}}\right)}=\frac{1}{1+9.14 \exp \left(-0.345 \mathrm{x}_{d}\right)}, \mathrm{x}_{d} \leq \frac{\text { channel width }}{2}
$$

For $\varphi=0.6$ :

$$
\begin{aligned}
& \overline{p_{s, \text { filtered }, \mathrm{xx}, \text { scaled }}}=\frac{\overline{p_{s, \text { filtered } \mathrm{xx}}}\left(\overline{\phi_{s}}, \mathrm{x}_{d}\right)}{\overline{p_{s, \text { filtered }, \mathrm{xx}, \text { core }}}\left(\overline{\phi_{s}}\right)} \\
& =\left\{\begin{array}{l}
-0.00267 \mathrm{x}_{d}{ }^{2}+0.0926 \mathrm{x}_{d}+0.180, \mathrm{x}_{d} \leq 14.5 \\
(1+25.4)\left(1-\exp \left(-0.450 \mathrm{x}_{d}\right)\right)-25.4,14.5<\mathrm{x}_{d} \leq \frac{\text { channel width }}{2}
\end{array}\right. \\
& \overline{p_{s, \text { filtered }, \mathrm{xx}, \text { core }}}\left(\overline{\phi_{s}}\right)=\overline{p_{s, \text { filtered, } \mathrm{xx}, \text { periodic }}}\left(\overline{\phi_{s}}\right)
\end{aligned}
$$

For $0<\varphi \leq 1$, we can capture the effect of $\varphi$ on the wall corrections with the correlation given below:

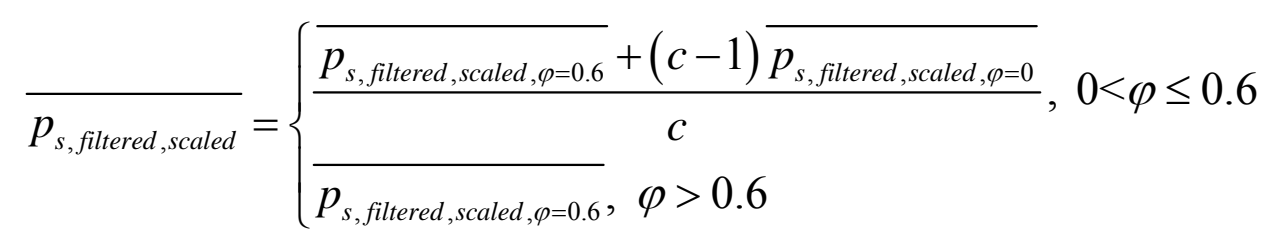

Here, "c" is defined as $0.6 / \varphi$. For instance, $\mathrm{c}=2$ for $\varphi=0.3$. 


\section{Filtered particle phase shear viscosity:}

For $\varphi=0.0001$ (free slip):

$$
\overline{\mu_{s, \text { filtered,scaled }}}=\frac{\overline{\mu_{s, \text { filtered }}}\left(\overline{\phi_{s}}, \mathrm{x}_{d}\right)}{\overline{\mu_{s, \text { filtered,core }}\left(\overline{\phi_{s}}\right)}}=\frac{1}{1+5.69 \exp \left(-0.228 \mathrm{x}_{d}\right)}, \mathrm{x}_{d} \leq \frac{\text { channel width }}{2}
$$

For $\varphi=0.6$ :

$\overline{\mu_{s, \text { filtered,scaled }}}=\frac{\overline{\mu_{s, \text { filtered }}}\left(\overline{\phi_{s}}, \mathrm{x}_{d}\right)}{\overline{\mu_{s, \text { filtered }, \text { core }}}\left(\overline{\phi_{s}}\right)}=(1-0.130)\left(1-\exp \left(-0.123 \mathrm{x}_{d}\right)\right)+0.130, \mathrm{x}_{d} \leq \frac{\text { channel width }}{2}$

$\overline{\mu_{\text {s, filtered,core }}}\left(\overline{\phi_{s}}\right)=1.15 \overline{\mu_{\text {s, filtered,periodic }}}\left(\overline{\phi_{s}}\right)$

For $0<\varphi \leq 1$, we can capture the effect of $\varphi$ on the wall corrections with the correlation given below:

$$
\overline{\mu_{s, \text { filtered ,scaled }}}=\left\{\begin{array}{l}
\overline{\overline{\mu_{s, \text { filtered }, \text { scaled }, \varphi=0.6}}+(c-1) \overline{\mu_{s, \text { filtered }, \text { scaled }, \varphi=0}}}, 0<\varphi \leq 0.6 \\
\overline{\mu_{s, \text { filtered,scaled }, \varphi=0.6}}, \quad \varphi>0.6
\end{array}\right.
$$

Here, "c" is defined as $0.6 / \varphi$. For instance, $\mathrm{c}=2$ for $\varphi=0.3$. 
APPENDIX C 
Closures for filtered two-fluid models for fluidized gas-particle suspensions

(draft manuscript)

by

Yesim Igci and Sankaran Sundaresan*

Department of Chemical Engineering, Princeton University, Princeton, NJ 08544

* Corresponding author: 1(609) 258-4583; SUNDAR@PRINCETON.EDU 


\begin{abstract}
In our earlier work, we have presented a methodology where computational results obtained through highly resolved simulations (in a large periodic domain) of a given microscopic two-fluid model are filtered to deduce closures for the corresponding filtered two-fluid model equations that are appropriate for coarse-grid simulations of gas-particle flows of moderate particle volume fractions. We have found that, to a good approximation, the dimensionless filtered drag coefficient, particle phase pressure and particle phase viscosity extracted from twoand three- dimensional simulations can be treated as functions of only particle volume fraction and dimensionless filter size.
\end{abstract}

However, the closure relationships for the drag coefficient and the effective stresses presented in our earlier work did not capture the behavior in regions of very low concentrations $\left(\phi_{s}<0.01\right)$ well and did not include high particle concentrations $\left(\phi_{s}>0.30\right)$ at all. In this study, we have investigated the region-averaged statistics in these regimes as well. We have also analyzed the extended data obtained over a wide range of filter sizes and scaled them into correlations, thereby shedding some light on the asymptotic behavior at large filters . 


\section{Introduction:}

In our earlier studies ${ }^{40}$, we outlined a systemic filtering approach and constructed closure relationships for the drag coefficient and the effective stresses in the gas and particle phases that are appropriate for coarse-grid simulations of gas-particle flows of moderate particle volume fractions. Briefly, we performed highly resolved simulations of a kinetic theory based two-fluid model with Wen \& Yu drag for uniformly sized particles ${ }^{2,30,31,40,50}$ in a large periodic domain (considerably larger than the filter size) and analyzed the results -obtained through a filtering operation which amounts to spatial averaging over some chosen filter length scale- using different filter lengths. In these filtered (a.k.a. coarse-grained) equations, the consequences of the flow structures occurring on a scale smaller than a chosen filter size appeared through residual correlations for which one must derive or postulate closure relations. We showed that the closure relationships for the drag coefficient and the effective stresses in the gas and particle phases manifested a definite and systematic dependence on the filter length (considerably smaller than the periodic domain size). Moreover, through a linear stability analysis of the filtered twofluid model equations, we showed that filtering did indeed remove small scale structures that were afforded by the highly resolved simulations of the kinetic theory based two-fluid model. However, the closure relationships for the drag coefficient and the effective stresses presented in our earlier work did not capture the regions of very low concentrations $\left(\phi_{s}<0.01\right)$ well and did not include high particle concentrations $\left(\phi_{s}>0.30\right)$ at all. In this study, we have investigated the region-averaged statistics in these phases as well. We have also analyzed the extended data obtained over a wide range of filter sizes and scaled them into correlations, thereby shedding some light on the asymptotic behavior at large filters . 
As noted in our earlier work ${ }^{40,}$ we restrict our attention to closures for $\overline{\beta_{\text {filtered }}}, \overline{p_{s, \text { filtered }}}$ and

$\overline{\mu_{s, \text { filtered }}}$ in flow regions far away from solid boundaries. A simple and effective manner by which solid boundaries can be avoided is to consider flows in periodic domains. The filtering operation does not require a periodic domain; however, as each location in a periodic domain is statistically equivalent to any other location, statistical averages can be gathered much faster when simulations are done in periodic domains. With this in mind, all the analyses described here have been performed in periodic domains. In our earlier work, we have already have already shown that the results obtained from 2-D and 3-D periodic domains are qualitatively similar, but differ somewhat quantitatively; therefore, we have focused first on 2-D simulations in the present study to bring forward the filter size dependence of the closures for the residual correlations, as 2-D simulations are computationally less expensive. We will present several 3-D simulation results at the end to bring forth the differences between 2-D and 3-D closures for the entire particle volume fraction range $\left(0<\phi_{s}<\phi_{s, \max }\right)$.

\section{Two-dimensional (2-D) simulations:}

To extract the constitutive relationships for 2-D filtered model equations for various filter lengths, we have performed many sets of highly resolved simulations of the set of microscopic two-fluid model equations ${ }^{30,40}$ in a $131.584 \times 131.584$ (dimensionless units) square periodic domain using the open-source software MFIX ${ }^{5}$; this domain size translates $0.64 \mathrm{~m} \mathrm{x} 0.64 \mathrm{~m}$ for the FCC particles (whose physical properties are given Table1). (Also, note that a dimensionless filter length of 2.056 corresponds to a filter length of $0.01 \mathrm{~m}$. In this case, the filter size is 2.056 x 2.056 (dimensionless units).) 


\section{2-D Filtered drag coefficient:}

Figure 1(a) shows the variation of the dimensionless filtered drag coefficient, $\overline{\beta_{\text {filtered }, d}}=\left(\overline{\beta_{\text {filtered }}} \mathrm{v}_{\mathrm{t}} / \rho_{s} g\right)$ as a function of particle volume fraction, $\phi_{s}$ for various filter lengths for the entire particle volume fraction range $\left(0<\phi_{s}<\phi_{s, \max }\right)$. (In all of our studies, $\phi_{s, \max }=0.65$.) The uppermost curve in Figure 1(a) is the intrinsic drag law; the filter length here is simply the grid length used in the simulations of the microscopic two-fluid model equations (which is equivalent to no filtering at all). For typical FCC particles (whose physical properties are given in Table 1), a dimensionless filter length of 2.056 is equivalent to $0.01 \mathrm{~m}$. It is apparent in Figure 1(a) that the filter length (or size) effect on the filtered drag coefficient depends on the particle concentration of a region.

To illustrate this effect more clearly, the results presented in Figure 1(a) are plotted in Figure 2(a) on a natural logarithmic scale. The figure clearly shows the effect of filter size on the filtered drag coefficient (from right to left) (a) at small $\phi_{s}$ values, (b) the typical RichardsonZaki $^{44}$ form for $\phi_{s}$ not too close to zero, presented in our earlier work ${ }^{40,}$ (c) at high $\phi_{s}$ values, a clear departure from this trend, and (d) at extremely high $\phi_{s}$ values, where filter size dependence disappears. (The uppermost curve in this figure corresponds to the intrinsic drag expression as in Figure 2(a). (We will turn back to this figure later for more discussion.)

To recap the results presented in our earlier manuscript, let us first focus our attention on the regions with $\phi_{s}<0.30$, shown in Figure 1(b) (also in Figure 2 (b) on a natural logarithmic scale). In this region, the filtered drag coefficient decreases substantially with increasing filter size, even at small filter sizes (from an engineering viewpoint). Here, we should note that the 
results for the two smallest filter sizes are likely to decrease somewhat if simulations could be done at higher resolutions, but as noted in our previous studies ${ }^{40}$; the results for all larger filter sizes are essentially independent of grid size.

The reduction in filtered drag coefficient with increasing filter size can be readily rationalized. As the filter size is increased, the averaging is being performed over larger and larger clusters - larger clusters allow greater bypassing of the gas resulting in lower apparent drag coefficient.

Turning our attention back to Figures $2(\mathrm{~b})$, we can readily see that all of the curves exhibit a Richardson-Zaki like behavior at moderate volume fractions and a reversal of trend at very low particle volume fractions. Linear fits of the data in Figure 2 (b) over the "moderate" particle volume fraction range $\left(0.05<\phi_{s}<0.30\right)$ were used to estimate dimensionless apparent terminal velocity $\mathrm{V}_{t, a p p}$ and an apparent Richardson-Zaki exponent, $N_{R Z, a p p} \cdot \mathrm{V}_{t, a p p}$ and $N_{R Z, a p p}$ are estimated from the y-intercept (at abscissa $=0$ ) and the slope of the straight line fit of the data on Figure 2 (a-b) (between abscissa values of -0.35 and -0.05$)$, respectively.

$$
\ln \left(\frac{\overline{\beta_{\text {filtered }}} \mathrm{V}_{\mathrm{t}}}{\rho_{s} g \phi_{s}\left(1-\phi_{s}\right)}\right)=\ln \left(\frac{\overline{\beta_{\text {filtered }, d}}}{\phi_{s}\left(1-\phi_{s}\right)}\right)=-\left(N_{R Z, a p p}-1\right) \ln \left(1-\phi_{s}\right)-\ln \left(\mathrm{V}_{t, a p p}\right)
$$

The variation of $\mathrm{V}_{t, a p p}$ and $N_{R Z, a p p}$ with dimensionless filter length, $F r_{f}^{-1}\left(=g \Delta_{f} / \mathrm{v}_{\mathrm{t}}^{2}\right)$, are shown in Figure 3 (a) (diamonds) and 3(b), respectively. Here $\Delta_{f}$ denotes the filter length. An identical analysis was presented in our earlier work and details will not be repeated here. However, it is important to note that there are some qualitative differences between the results presented in our earlier work and the current one. These differences result from the fact that we 
have collected more data in an extended particle concentration range and improved the constitutive relationships for the filtered quantities in this study.

Now focusing our attention on low particle concentration regions (this corresponds to the right-most region in the figure), we can readily notice that the Richarson-Zaki like model (represented with solid lines in Figure 2(b)) for the filtered drag coefficient does not capture the region of low particle concentration (typical of freeboard region a turbulent bed) and a qualitatively different dependence of the filtered drag coefficient on particle volume fraction is obtained. The variation of the (dimensionless) filtered drag coefficient as a function of filtered (i.e. region-averaged) particle volume fraction for various filter lengths in the dilute region is illustrated in Figure 1(c). This figure reveals two interesting trends:

a) At extremely low particle volume fractions all the curves corresponding to various filter sizes approach the same asymptotic behavior indicating that the filtered drag coefficient is essentially independent of filter size in this region.

b) As the particle volume fraction increases, the filtered drag coefficient picks up a definite dependence on the filter size, manifesting a systematic decrease with increasing filter size.

It is apparent from the figure that one has to go down to extremely low particle volume fractions $\left(\sim 10^{-4}\right)$ before the filter size dependence becomes really weak. Thus, for practical purposes, the filter size dependence can be taken as persistent at regions of low particle concentration as well.

In our earlier work ${ }^{40,}$ we have investigated the reason behind the qualitatively different dependence of the filtered drag coefficient on particle volume fraction in the regions with low 
particle concentrations. We have concluded that this behavior is not due to an $\operatorname{Re}_{g}$ effect in the intrinsic drag law and have sought an alternate explanation. We have attributed the trend reversal seen in Figures 2(a) and 2(b) at small $\phi_{s}$ values to just the inhomogeneous microstructure inside the filter region. At low $\phi_{s}$ values, an increase in $\phi_{s}$ increases both the cluster size and particle volume fraction in the clusters; the gas flows around these clusters and the resistance offered by these clusters decreases with increasing cluster size. Large filter sizes average over larger clusters and so the extent of drag reduction observed increases with filter size. At sufficiently large $\phi_{s}$ values, the clusters begin to interact and hindered drag sets in. This behavior is clearly reflected in the vertical slip velocity corresponding to large filter sizes, see Figure 4 . The slip velocity increases with $\phi_{s}$ at small $\phi_{s}$ values, consistent with larger and/or denser clusters; it then decreases with increasing $\phi_{s}$ when the clusters begin interact with each other.

Next, focusing our attention on the region of high particle volume fraction $\left(\phi_{s}>0.30\right)$ in Figure 2(a) (this corresponds to the left-most region in the figure), we can readily see that the filtered drag coefficient in this region also shows a clear departure from the Richardson-Zaki like behavior. (The 2-D dimensionless filtered drag coefficient is presented in Figure 1(a)). Figure 2(a) reveals some interesting trends:

a) For $\phi_{s}>0.30$, the 2-D filtered drag coefficient cannot be captured with a Richarson-Zaki like model. However, the dependence of the filtered closures on filter length is still clearly observed for $0.30<\phi_{s}<0.59$. Note that $\phi_{s} \square 0.30$ value coincides with the transition of the meso-scale structures from clusters and strands (elongated structures of high particle concentration) to voids (bubbles). 
b) At extremely high particle volume fractions (for $\phi_{s}>0.59$ ) all the curves corresponding to various filter sizes approach the same value indicating that the filtered closures are essentially independent of filter length in this region.

c) The filtered drag coefficient (for all filter lengths) value at extremely high particle concentrations correspond to those used in the microscopic model ${ }^{30,40 .}$

As noted earlier, at sufficiently large $\phi_{s}$ values, the clusters begin to interact and hindered drag sets in. At $\phi_{s} \approx 0.30$, the interaction between the meso-scale structures leads to a transition from clusters and strands (elongated structures of high particle concentration) to voids (bubbles). This turbulent state is suspected to cause the departure from the Richarson-Zaki like behavior. The effect of filtering remains unchanged for $0.30<\phi_{s}<0.40$. However, for $\phi_{s}>0.40$, the effect of filtering starts to decrease as particle concentration increases (leading to more interaction between structures). Finally, at very high $\phi_{s}(>0.59)$ values (close to $\phi_{s}$ at maximum packing), most regions with such high particle concentrations are devoid of voids (bubbles) and behave as nearly homogeneous systems close to maximum packing limit, which are captured by the microscopic model. This behavior is also reflected in the vertical slip velocity corresponding to high $\phi_{s}$ values, see Figure 4. (At at high $\phi_{s}$ values, all filtered vertical slip velocity curves overlap with the curve obtained from a filter length equal to the grid length used in the highlyresolved kinetic theory simulations.)

Before we proceed any further with modeling the filtered drag coefficient, we should note that we have extracted various scaling characteristics through an analysis of the data (for $0 \leq \phi_{s}<0.65$ ) obtained over a wide range of filter sizes; such an analysis has shed some light on the asymptotic behavior at large filter sizes (as discussed below). 


\section{Scaling behavior of the filtered drag coefficient:}

From the dimensionless results presented in Figures 1(a-c), one can extract various scaling characteristics. In all our studies, the dimensionless filter length is defined as $F r_{f}^{-1}=g \Delta_{f} / v_{t}^{2}$. For $F r_{f}^{-1} \geq 4.112$, the filtered drag coefficient was found to vary roughly as $\left(F r_{f}\right)^{m\left(F r_{f}^{n}\right)}$. Here, "m" and "n" are functions of particle volume fraction and are given in Figure 5. Using the 2-D filtered drag coefficient for a filter length of 4.112 and this scaling correlation, we have obtained the filtered drag coefficients for various large filter lengths which cannot be obtained with the simulation domain used in these studies. For instance, to obtain the filtered drag coefficient for a dimensionless filter length of $16.448\left(\overline{\beta_{\text {filtered,Fr-1 }}=16.448}\right)$, we have used the following relationship:

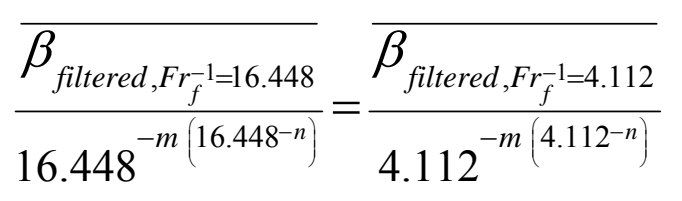

One can expect that the clusters will not grow beyond some critical size and that at sufficiently large filter sizes the filtered drag coefficient will become essentially independent of the filter size. The current scaling analysis predicts the asymptotic value of the filtered drag coefficient at large filter sizes. We will use this asymptotic value in modeling the filtered drag coefficient in an internally consistent manner. Details will be given later.

Similarly, we have also found that for $\mathrm{Fr}_{f}^{-1} \geq 4.112$, the filtered particle phase pressure and viscosity varied as $\left(F r_{f}\right)^{p}$ and $\left(F r_{f}\right)^{q}$, respectively. The variation of exponents "p" and "q" 
with particle volume fraction are shown in Figure 6. Using these scaling correlations and the filtered stress terms extracted with a filtered size of 4.112, we obtained the 2-D filtered stress terms for various large filter sizes that cannot be obtained with the simulation domain used in these studies. We will use these results in the next section, where we will discuss the 2-D filtered particle phase stresses.

One weakness of this type of scaling is that it cannot capture the 2-D filtered model closures for $\mathrm{Fr}_{f}^{-1}<4.112$. In addition, to be internally consistent, the filtered model closures must approach the microscopic model closures as $\mathrm{Fr}_{f}^{-1} \rightarrow 0$. In order to remedy this weakness, we have sought a slightly different scaling. This new scaling utilizes the results obtained from the scaling described above for large filter sizes.

Turning our attention to the filtered drag coefficient in the regions with $0.01<\phi_{s}<0.30$, we have sought a scaling correlation in the form of an arithmetic weighted sum of the models capturing the asymptotic value of R-Z like region (Function ${ }_{\text {asymptotic }}$ ) and that used in the

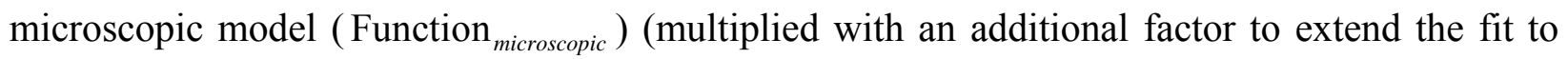
low volume fraction region):

Function $_{\text {moderate }}=\left(\right.$ a Function $_{\text {asymptotic }}+(1-a)$ Function $\left._{\text {microscopic }}\right)\left(1-0.606\left(1-\phi_{s}\right)^{125}\right)$

Function $_{\text {asymptotic }}=\ln \left(\frac{\overline{\beta_{\text {asymptotic }}} \mathrm{V}_{\mathrm{t}}}{\rho_{s} g \phi_{s}\left(1-\phi_{s}\right)}\right)=-4.19 \ln \left(1-\phi_{s}\right)-1.73$ for $0.05<\phi_{s}<0.30$

where " $a$ " is the weighting function and is shown in Figure 7 (a), as a function of dimensionless filter length. "a" takes values between zero and one. When "a" is equal to zero, the model 
captures the microscopic model, and when it is equal to one, it captures the asymptotic model for large filters. The asymptotic value was obtained through the scaling studies described earlier. (It should be noted that the value of "a" is expected to increase slightly with resolution for filter sizes smaller than 1.028 dimensionless units.) As noted earlier, the filtered drag coefficient decreases with increasing filter size; this behavior is captured with filter size dependence of "a", which can be modeled as:

$$
a=\left\{\begin{array}{l}
-0.0350 F r_{f}^{-2}+0.300 F r_{f}^{-1}, F_{f}^{-1}<4.00 \\
-1.44 F r_{f}+1.00, F_{f}^{-1} \geq 4.00
\end{array}\right.
$$

Function $_{\text {microscopic }}$ corresponds to natural logarithmic value of the filtered drag coefficient extracted with a filter size equal to the grid size used in the 2D kinetic theory based simulations with Wen-Yu drag; the grid length is 0.257 dimensionless units. The equations given below are the simplest fits that capture the curve reasonably well for the rest of our studies.

$$
\begin{aligned}
\text { Function }_{\text {microscopic }}= & \ln \left(\frac{\overline{\beta_{\text {microscopic }}} \mathrm{v}_{\mathrm{t}}}{\rho_{s} g \phi_{s}\left(1-\phi_{s}\right)}\right) \\
= & \left\{\begin{array}{l}
0.230\left(1-\phi_{s}\right)^{76.6}+0.0914\left(1-\phi_{s}\right)^{9770}, \phi_{s}<0.001 \\
0.180\left(1-\phi_{s}\right)^{-10.6}+0.0720\left(1-\phi_{s}\right)^{800}, 0.001 \leq \phi_{s}<0.03 \\
0.550\left(\ln \left(1-\phi_{s}\right)\right)^{2}-2.82 \ln \left(1-\phi_{s}\right)+0.162, \phi_{s} \geq 0.03
\end{array}\right.
\end{aligned}
$$

Next, we will focus on modeling the dense region $\left(\phi_{s}>0.30\right)$ in the form of a function which combines the moderate and high particle volume fraction regions (in Figure 2 (a)). Here, we have modeled $\ln (\mathrm{Q})\left(Q=\frac{\overline{\beta_{\text {filtered } d}}}{\phi_{s}\left(1-\phi_{s}\right)}\right)$ as an arithmetic weighted sum of the models capturing 


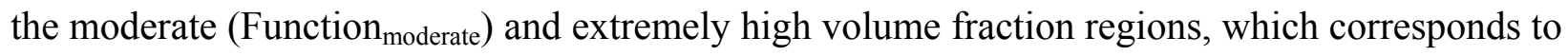
that used in the microscopic model (Function microscopic $_{\text {): }}$

Function $_{\text {moderate-dense }}=$ Function $_{\text {moderate }}$ factor $_{\text {dense }}+$ Function $_{\text {microscopic }}\left(1-\right.$ factor $\left._{\text {dense }}\right)$

where factor $_{\text {dense }}$ is the weighting function and is defined as:

$$
\text { factor }_{\text {dense }}=\frac{1}{1+1.23 \times 10^{-4}\left(1-\phi_{s}\right)^{-15.5}}
$$

Lastly, we have investigated a model for the filtered drag coefficient in the very low volume fraction region (which was discussed earlier) and tied it to the rest through a function of the form:

$$
\begin{aligned}
\text { Function }_{\text {total }} & =\ln \left(\overline{\frac{\beta_{\text {filtered,d }}}{\phi_{s}\left(1-\phi_{s}\right)}}\right) \\
& =\text { Function }_{\text {dense-moderate }}\left(1-\text { factor }_{\text {dilute }}\right)+\text { Function }_{\text {microscopic }} \text { factor }_{\text {dilute }}
\end{aligned}
$$

Function $_{\text {total }}$ is an arithmetic weighted sum of the functions capturing the Richardson-Zaki like region and that for the microscopic model (Function $\left._{\text {microscopic }}\right)$. factor $_{\text {dilute }}$ is the weighting function:

$$
\text { factor }_{\text {dilute }}=\left(1-\phi_{s}\right)^{b}
$$

A function in this form would ensure that as $\phi_{s} \rightarrow 0$, all the curves would tend to the

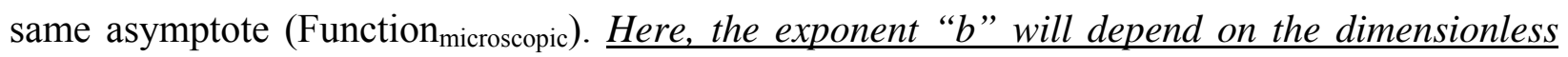
filter length. The systematic variation of the exponent " $b$ " with filter length is shown in Figure 7(b). It is clearly seen that "b" first increases with the filter length and then reaches an 
asymptotic value of 1440 at large filters. It is also important to note that Function total $_{\text {in }}$ is equal to Function $_{\text {microscopic }}$ when "b" is zero. "b" can be modeled with the function given below (represented by a dashed line in Figure 7(b)):

$$
b=\left\{\begin{array}{l}
292 F r_{f}^{-1}, F r_{f}^{-1}<0.500 \\
\frac{1440}{1+\exp \left(-0.560 F r_{f}^{-1}+2.47\right)}, F r_{f}^{-1} \geq 0.500
\end{array}\right.
$$

Figures 8 (a) and 8(b) present comparisons of the fits in the form of Function total $_{\text {with }}$ the computational data on a logarithmic scale for the entire particle volume fraction range and only in the dilute region, respectively. It is apparent from the figures expression of the form given by Function $_{\text {total }}$ does indeed capture the filtered drag coefficient data reasonably well.

The proposed constitutive relationships for the 2-D filtered drag coefficient that cover the entire range of particle volume fractions observed are summarized in Table 2. Using these constitutive relationships, one can easily obtain the filtered drag coefficient for any filter size as defined below:

$$
\overline{\beta_{\text {filtered }}}=\frac{\left(\exp \left(\text { Function }_{\text {total }}\right)\right) \rho_{s} \phi_{s}\left(1-\phi_{s}\right)}{\mathrm{v}_{\mathrm{t}}}
$$

\section{2-D Filtered particle phase pressure and viscosity:}

The filtered particle phase pressure $\left(\overline{p_{s, f i t e r e d}}\right)$ and shear viscosity $\left(\overline{\mu_{s, \text { filtered }}}\right)$ include the stress terms arising from the streaming and collisional parts captured by the kinetic theory and the subfilter-scale Reynolds-stress like velocity fluctuations . In this section, we will seek closure relations for $\overline{p_{s, \text { filtered }}}$ and $\overline{\mu_{s, \text { filtered }}}$ as a function of particle volume fraction and filter length. 


\section{Modeling the filtered particle phase pressure:}

The dimensionless filtered particle phase pressure was defined as $\overline{p_{s, \text { filtered }, d}}\left(=\left(\overline{p_{s, \text { kinetic }}}+\frac{1}{3}\left(\rho_{\mathrm{s}} \overline{\phi_{s} \mathrm{v}_{\mathrm{x}}^{\prime} \mathrm{v}_{\mathrm{x}}^{\prime}}+\rho_{\mathrm{s}} \overline{\phi_{\mathrm{s}} \mathrm{v}_{\mathrm{y}}^{\prime} \mathrm{v}_{\mathrm{y}}^{\prime}}+\rho_{\mathrm{s}} \overline{\phi_{\mathrm{s}} \mathrm{v}_{\mathrm{z}}^{\prime} \mathrm{v}_{\mathrm{z}}^{\prime}}\right)\right) / \rho_{\mathrm{s}} \mathrm{v}_{\mathrm{t}}^{2}\right)$. Here, $\overline{p_{s, \text { kinetic }}}$ is the filtered pressure arising from the streaming and collisional parts captured by the kinetic theory. Figure 9 (a) shows the variation with $\phi_{s}$ of $\overline{p_{s, k i n e t i c, d}}\left(=\overline{p_{s, k i n e t i c}} / \rho_{s} v_{t}^{2}\right)$ over the entire particle volume fraction range $\left(0<\phi_{s}<0.65\right)$, for the simulations discussed earlier in connection with Figure 1(a-c). To show the effect of filtering more clearly in the regions with moderate particle concentration, we have also included Figure 9(b), showing the same plot for $\phi_{s}<0.59$. At very low $\phi_{S}$ values the filtered kinetic theory pressure is essentially independent of filter length, but at moderate $\phi_{s}$ values filter length dependence becomes clear. However, the filter size dependence disappears at high $\phi_{s}$ values $\left(\phi_{s}>0.50\right)$.

It is important to note that filtered kinetic theory model pressure initially increases with particle concentration and reaches a local maximum value (at $\left.\phi_{s} \approx 0.30\right)$. Then, it starts to decrease with increasing particle concentration until it reaches a local minimum value at $\phi_{s} \approx 0.59$ After this point, the kinetic theory pressure increases rapidly because of the radial distribution term for the particle phase, which diverges as $\phi_{s}$ increases.

Figure 10 (a) shows the dimensionless total filtered particle phase pressure as a function of $\phi_{s}$ for various filter sizes over the entire particle volume fraction range $\left(0<\phi_{s}<0.65\right)$. Here the filtered particle phase pressure includes the filtered kinetic theory model pressure and the subfilter-scale Reynolds-stress like velocity fluctuations. (The filtered particle phase pressure for a 
filter length equal to the grid length corresponds to the kinetic theory pressure.) Comparing Figures 9 (a) and Figure 10(a), it readily becomes apparent that the contributions resulting from the sub-filter-scale velocity fluctuations swamp the kinetic theory pressure for $\phi_{s}<0.59$ indicating that, at the coarse-grid scale, one can ignore the kinetic theory contributions to the filtered particle phase pressure for $\phi_{s}<0.59$. At higher $\phi_{s}$ values, the filtered kinetic theory contributions becomes the dominant term. We will explain this behavior at high $\phi_{S}$ values later in this section.

It is readily seen in Figure 10 (a) that the filtered particle phase pressure increases with filter length for $\phi_{s}<0.59$, a direct consequence of the fact that the energy associated with the velocity fluctuations increases with filter length (as in single phase turbulence). And, for a given filter length, the filtered particle phase pressure increases with particle volume fraction within the particle volume fraction range of $0 \leq \phi_{s}<0.28$. The physical explanation for this trend is that that the greater the solid concentration is the larger the velocity fluctuations are. As discussed earlier, the contributions resulting from the sub-filter-scale velocity fluctuations swamp the kinetic theory pressure in this region. For $\phi_{s}>0.28$, the behavior of the 2-D filtered particle phase pressure as a function of particle volume fraction and filter size can be divided into two main regions. In the first region, for $0.28<\phi_{s}<0.59$, the filtered particle phase pressure increases with filter length. However, for a given filter length, it decreases as the particle volume fraction in a region increases. This trend is the opposite of what occurs in regions with $0 \leq \phi_{s}<0.28$. The physical explanation for this behavior is that, over a certain limit of particle concentration (about 0.28), greater particle concentration starts to restrict velocity fluctuations in a region instead of increasing it. Eventually, for $\phi_{s}>0.59$, the physical oscillations of clusters get restricted to the 
point that both meso-scale stress terms disappear, and only the kinetic model particle stress terms remain. Consequently, for $\phi_{s}>0.59$, both filtered quantities are nearly independent of the filtered length.

We have sought an internally consistent model that combines the three regions, namely the region with low, moderate, and high particle volume fractions, in a compact manner (as a function of particle volume fraction and filter length). We have modeled the 2-D dimensionless filtered particle phase pressure as:

$$
\frac{\overline{p_{s, \text { fitered }}}}{\rho_{s} \mathrm{v}_{\mathrm{t}}^{2}}=\left\{\begin{array}{l}
\frac{\overline{p_{s, \text { kinetic }}}}{\rho_{s} \mathrm{v}_{\mathrm{t}}^{2}}+\text { Factor }_{p s} \phi_{s}\left(1+\phi_{s}-11 \phi_{s}^{2}+10.9 \phi_{s}^{3}\right), \phi_{s} \leq \phi_{s, \text { rritical_l }} \\
\frac{p_{s, \text { kinetic }}}{\rho_{s} \mathrm{v}_{\mathrm{t}}^{2}}, \phi_{s}>\phi_{s, \text { rritical } \_1}
\end{array}\right.
$$

where $\phi_{s, \text {,ritical__l }}(\approx 0.59)$ is defined as:

$$
\text { Factor }_{p s} \phi_{s}\left(1+\phi_{s}-11 \phi_{s}^{2}+10.9 \phi_{s}^{3}\right)=0 \text { at } \phi_{s}=\phi_{s, \text { critical } \_1}
$$

Figure 10(b) shows the variation of Factor ${ }_{p s}$ as a function of dimensionless filter size. It is readily seen that Factor ${ }_{p s}$ increases with filter size and does not reach any asymptotic values. The data presented in Figure 10(b) can be curve-fitted as

$$
\text { Factor }_{p s}=\left\{\begin{array}{l}
0.0300 F r_{f}^{-2}+0.250 F r_{f}^{-1}, F r_{f}^{-1}<4.80 \\
0.438 F r_{f}^{-0.881}+0.138, F r_{f}^{-1} \geq 4.80
\end{array}\right.
$$

This model is internally consistent; it will approach the kinetic model particle phase stress terms as $\mathrm{Fr}_{f}^{-1} \rightarrow 0$. The kinetic model term in these expressions can be taken care of in two ways:

1. Also solve the granular energy equation (which is granular temperature dependent). 
2. Simply add the curve fit (given below) for the kinetic particle phase pressure.

$$
\frac{\overline{p_{s, \text { kinetic }} \mathrm{v}^{2}}}{\rho_{\mathrm{s}} \mathrm{v}_{\mathrm{t}}^{2}}=\left\{\begin{array}{l}
-10.4 \phi_{s}^{2}+0.310 \phi_{s}, \phi_{s} \leq 0.0131 \\
-0.185 \phi_{s}^{3}+0.0660 \phi_{s}^{2}-0.000183 \phi_{s}+0.00232,0.0131<\phi_{s} \leq 0.290 \\
-0.00978 \phi_{s}+0.00615,0.290<\phi_{s} \leq 0.594 \\
-6.62 \phi_{s}^{3}+49.5 \phi_{s}^{2}-50.3 \phi_{s}+13.8, \phi_{s}>0.594
\end{array}\right.
$$

This curve-fit model for the kinetic theory pressure is a granular temperature independent approximation and does not require solving the full kinetic theory based two-fluid model. In these expressions, we have ignored the effect of filtering on the kinetic model pressure, which is negligible compared to the effect on the Reynolds-like terms and used the filtered kinetic theory pressure obtained with a filter length equal to the grid length.

As noted earlier, using these correlations, one can easily obtain the filtered particle phase pressure for any filter length. Figure 10 (a) also includes the model described above for various filter lengths. A comparison of these fits with the computational data reveals that the model captures the filtered particle phase pressure reasonably well. (The proposed constitutive relationships that cover the entire range of particle volume fractions observed are summarized in Table 2).

Modeling the filtered particle phase shear viscosity:

Analogous to the modeling studies of the filtered particle phase pressure, we have defined that the dimensionless (total) filtered particle phase shear viscosity as $\overline{\mu_{s, \text { filtered }}}=\overline{\mu_{s, \text { kinetic }}}+\rho_{\mathrm{s}} \overline{\phi_{\mathrm{s}} \mathrm{v}_{\mathrm{x}}^{\prime} \mathrm{v}_{\mathrm{x}}^{\prime}} /\left(\frac{\overline{\partial \mathrm{v}_{\mathrm{x}}}}{\partial y}+\frac{\overline{\partial \mathrm{v}_{\mathrm{y}}}}{\partial x}\right)$. Here $\overline{\mu_{s, k i n e t i c}}$ is the filtered viscosity arising from the streaming and collisional parts captured by the kinetic theory. Figure 11 (a) shows the variation 
with $\phi_{s}$ of $\overline{\mu_{s, k i n e t i c, d}}\left(=\overline{\mu_{s, k i n e t i c}} / \rho_{s} v_{t}^{3}\right)$ over the entire particle volume fraction range ( $0<\phi_{s}<0.65$ ), for the simulations discussed earlier in connection with Figure 1(a-c). To show the effect of filtering more clearly in the regions with moderate particle concentration, we have also included a figure showing the same plot for $\phi_{s}<0.30$. See Figure 11 (b). At all $\phi_{s}$ values $\overline{\mu_{s, k i n e t i c, d}}$ can be considered to be independent of filter length although a slight dependence on $\phi_{s}$ is observed at moderate $\phi_{s}$ values. As noted previously in the results for the kinetic theory pressure term, the kinetic theory viscosity increases rapidly at high $\phi_{s}$ values because of the radial distribution term for the particle phase, which diverges as $\phi_{s}$ increases.

Figure 12 (a) shows the dimensionless total filtered particle phase shear viscosity, $\overline{\mu_{s, \text { filtered }, d}}\left(=\overline{\mu_{s, \text { filtered }}} / \rho_{s} \mathrm{v}_{\mathrm{t}}^{3}\right)$, as a function of $\phi_{s}$ for various filter sizes over the entire particle volume fraction range $\left(0 \leq \phi_{s}<0.65\right)$. Here the filtered particle phase viscosity includes the filtered kinetic theory model viscosity and the sub-filter-scale Reynolds-stress like velocity fluctuations. (The filtered particle phase viscosity for a filter size equal to the grid size corresponds to the kinetic theory viscosity.) Analogous to what we have discussed in modeling the filtered particle phase pressure, a comparison of Figure 11 (a) and Figure 12 (a) reveals that the contributions resulting from the sub-filter-scale velocity fluctuations dominate and the filtered particle phase viscosity increases appreciably with filter size for $\phi_{s}<0.59$ indicating that, at the coarse-grid scale, one can ignore the kinetic theory contributions to the filtered particle phase viscosity for $\phi_{s}<0.59$. At higher $\phi_{s}$ values, the sub-filter-scale velocity fluctuations disappear (the physical oscillations of clusters get restricted), and only the kinetic model 
viscosity remains. As a result, the filtered particle phase viscosity becomes independent of the filtered size at extremely high $\phi_{s}$ values.

The variation of the filtered particle phase viscosity with increasing $\phi_{s}$ for a given filter size (larger than the grid size) is essentially the same as that of the filtered particle phase pressure. Therefore, we will not repeat it here. Based on this similarity, we have used similar constitutive relationships to capture the filtered particle phase shear viscosity and the filtered particle phase pressure. The constitutive relationships for the filtered particle phase pressure were discussed earlier, and those for the filtered particle phase shear viscosity are summarized in Table 2. A comparison of the fits for various filter lengths with the computational data reveals that the model captures the filtered particle phase pressure reasonably well; see Figure 12 (a).

\section{Three-dimensional (3-D) simulations:}

The analysis presented so far was restricted to two dimensions. To be of practical value, this type of analysis must be carried out in three dimensions. The 3-D filtered results presented in this section are based on computational data gathered in a $16.448 \times 16.448 \times 16.448$ (dimensionless units) cubic periodic domain; this domain size translates to $0.08 \mathrm{~m} \mathrm{x} 0.08 \mathrm{~m} \times 0.08 \mathrm{~m}$ for the FCC particles (whose physical properties are given Table1). Although the length of the domain is much smaller than that used in 2-D simulations (due to computational limitations), as we will show later, the domain size did not affect the filtered quantities as long as the filter length is much smaller than the domain dimensions. A dimensionless filter length of 2.056 corresponds to a filter length of $0.01 \mathrm{~m}$ for the $75 \mu \mathrm{m}$ FCC particles in ambient air.

\section{3-D Filtered Drag Coefficient:}


Figure 13(a) presents the variation of the (dimensionless) filtered drag coefficient, as a function of filtered particle volume fraction for various filter lengths over the entire particle volume fraction range $\left(0<\phi_{s}<0.65\right)$ observed in the simulations. As in 2-D simulations, it is clearly seen that the filter size effect on the filtered drag coefficient depends on the particle concentration of a region.

To illustrate this effect more clearly, as in the 2-D case, the results presented in Figures 13 (a-b) are plotted on a logarithmic scale in Figure 14 (a-b). The figures clearly show that the overall trend is qualitatively similar to that in 2-D. To illustrate the difference and similarities between the filtered drag coefficients extracted from the 2-D and 3-D simulations more explicitly, we have compared them for a filter size of 2.056 (dimensionless units) on the same figure. Figure 15 (a) compares $Q\left(=\overline{\beta_{\text {filtered }, d}} / \phi_{s}\left(1-\phi_{s}\right)\right)$ (on a logarithmic scale) extracted from 2D simulations (represented by grey filled triangles) with that extracted from 3-D simulations (represented by black stars). The overall trend in both 2-D and 3-D simulations is qualitatively similar. However, there are definite quantitative differences between 2-D and 3-D results. At moderate $\phi_{s}$ values, the filtered drag coefficient from the 3-D simulations is lower than that extracted from the 2-D simulations. This result was expected since 3-D inhomogeneous structures, in the form of 3-D globular and filaments allow the gas bypass these structures more than the 2-D clusters and streamers, which take the form of cylinders and sheets if extended to 3 D. However, the difference diminishes at very low and high particle volume fractions; the 2-D and 3-D filtered drag coefficients appear to converge.

Scaling behavior of the 3-D filtered drag coefficient: 
We have extracted various scaling characteristics for the 3-D filtered quantities through an analysis of the data (for $0 \leq \phi_{s}<0.65$ ) obtained over a wide range of filter sizes. This analysis is analogous to that described earlier in 2-D simulation results. Here, we will not get into details, but briefly state that such an analysis has shed some light on the asymptotic behavior at large filters. Figure 15 (b) presents a comparison of the asymptotic value of $Q\left(=\overline{\beta_{\text {filtered }, d}} / \phi_{s}\left(1-\phi_{s}\right)\right)$ (on a logarithmic scale) (for large filter sizes) extracted from 2-D simulations (represented with a solid grey line) with that extracted from 3-D simulations (represented with a dashed black line). The overall trend in both 2-D and 3-D simulations is qualitatively similar. However, the 2-D asymptotic value filtered drag coefficient is lower than the 3 -D one for $\phi_{s}<0.20$. This is exactly opposite of what we have observed with smaller filter sizes. We suspect that this change in behavior stems from that, at large filter sizes, the 3-D large meso-scale structures interact and hinder the gas bypassing the structures more than the 2-D clusters (consider one large cluster being surrounded by larger clusters like itself in three directions rather than in two directions.)

Utilizing the asymptotic value for the filtered drag coefficient, we have modeled the dimensionless 3-D filtered drag coefficient in the same way that we have modeled the 2-D filtered drag coefficient. The proposed constitutive models for the 3-D filtered drag coefficient are summarized in Table 3. Figures 16 (a) and (b) show the weighting function for the dense region, "a", and the exponential of the weighting function for the dilute region, "b", respectively.

Using these constitutive relationships, one can easily obtain the filtered drag coefficient for any filter size. Figures 17(a) and (b) present comparisons of the fits in the form of Function $_{\text {total }}$ with the computational data on a logarithmic scale for the entire particle volume fraction range and only in the dilute region, respectively. It is readily apparent from the figures 
that a constitutive relationship of the form given by Function $_{\text {total }}$ does indeed capture the 3-D filtered drag coefficient data reasonably well; see Table 3.

An alternate approach based on the aforementioned Energy Minimization Multi-Scale (EMMS) model (which assumes that the particles reside in a clustered state) has been developed by Li, Kwauk, and coworkers. ${ }^{51-55}$ The EMMS model and its extension (to cope with transient flow), EMMS/matrix ${ }^{56}$ are reported to have good success in capturing experimental data. ${ }^{57,58}$ Unlike the present study where the corrections to the drag force depend on filter length, the EMMS and EMMS/matrix models prescribe a fixed modification to the drag force, and it may perhaps be viewed as the large filter length limit. To illustrate this, we have compared the heterogeneity index, $H_{D}\left(=\beta_{\text {effective }} / \beta_{\text {Wen \& Yu }}\right)$, from EMMS/matrix model and from the asymptotic value for 3-D filtered drag coefficient (for very large filters). See Figure 18. Here, we have used Figure 1 from Lu et al. ${ }^{59}$, which shows the projected area plot of $H_{D}$ (a function of $\phi_{s}$ and $v_{\text {slip }}$ ) from the EMMS/matrix model (Model M), $H_{D}$ from the previous version of EMMS model (Model Y), and that from the Ergun/Wen-Yu (Model G) and incorporated our filtered 3$\mathrm{D}$ model in the figure for comparison. Wen and $\mathrm{Yu}$ drag coefficient was used as the standard drag coefficient, and the remaining models were scaled with it to get the corresponding $H_{D}$. Lu et al. ${ }^{59}$ state that $H_{D}$ from the EMMS/matrix model is a function of a function of $\phi_{s}$ and $v_{\text {slip }}$, and higher slip velocity generally results in higher values of $H_{D}$. The area plot shown in Figure 18 covers a range of $0.001<V_{\text {slip }}<15.2 \mathrm{~m} / \mathrm{s}$. In our 3-D simulations, the vertical slip velocity decreases from $1.0 \mathrm{~m} / \mathrm{s}$ to $0.40 \mathrm{~m} / \mathrm{s}$ as the particle volume fraction increases from 0.01 to 0.30 . Within this particle concentration and slip velocity range, the particle volume fraction 
dependence of $H_{D}$ from the EMMS/matrix model is qualitatively similar to that from 3-D filtered drag coefficient.

Some authors ${ }^{56,59-64}$ have combined EMMS model and its derivative EMMS/matrix model for drag with kinetic theory model for stresses ${ }^{65}$ (which assumes that individual particles

move chaotically), ${ }^{2,15}$ which appear to us to be incompatible; in contrast, the approach pursued in our studies filters the stresses and the drag in a systematic and consistent manner.

\section{3-D Filtered particle phase pressure and viscosity:}

We will now present the effect of the filter size and particle volume fraction on the 3-D filtered particle phase pressure and shear viscosity and derive constitutive relationships which capture these effects. The effect of filtering on the dimensionless filtered particle phase pressure and shear viscosity in the particle volume fraction range of $0<\phi_{s}<0.65$ are illustrated in Figure 19 (a) and Figure 20 (a), respectively. The trends are qualitatively similar to those obtained from the 2-D simulations.

To illustrate the difference and similarities between the 2-D and 3-D results more explicitly, we have compared them for a filter length of 2.056 (dimensionless units) on the same figure. Figure 21 (a) compares the filtered particle phase pressure extracted from 2-D simulations (represented with grey filled triangles) with that extracted from 3-D simulations (represented with black stars). As noted earlier, the strong filter size dependence of these quantities is clearly present in both 2-D and 3-D. However, for $\phi_{s}<0.2$, the filtered drag coefficient from the 3-D simulations is slightly larger than that extracted from the 2-D simulations. This trend is reversed at higher particle concentrations, which means the 2-D pressure is larger than that extracted from 
3-D simulations. This reversal in difference originates from the fact that 3-D vertical pressure is much larger than that in 2-D at $\phi_{s}<0.2$. As for the horizontal components, the 3-D lateral pressure is noticeably lower than that in 2-D for the entire particle volume range. The difference is more pronounced for larger filters.

Similarly, Figure 21 (b) compares the filtered particle phase shear viscosity extracted from 2-D simulations (represented by grey filled triangles) with that extracted from 3-D simulations (represented by black stars). It is clearly seen that the 2-D result is much higher than that from 3-D simulations. The difference is pronounced for larger filters. Here, it is important to note that the sub-filter-scale velocity fluctuations disappear at a lower $\phi_{s}$ value in 3-D simulations than they did in 2-D simulations. We attribute the difference between the filtered stress terms extracted from the 2-D and 3-D simulations to that the physical oscillations of clusters get more restricted in 3-D systems, particularly at higher particle volume concentrations.

Finally, we have sought internally consistent constitutive relationships for the 3-D filtered particle phase pressure and shear viscosity (as a function of particle volume fraction and filter size) which capture the trends discussed above. We have followed the same methodology that we used for the 2-D simulation results and modeled the stress terms in a similar way. The resulting constitutive relationships for the filtered particle phase pressure and shear viscosity are summarized in Table 3 and are included in Figure 19 (a) and Figure 20 (a), respectively. It is clearly seen that the proposed relationships capture the computational data reasonably well.

\section{Summary:}

In our earlier work, we have presented a methodology where computational results obtained through highly resolved simulations (in a large periodic domain) of a given microscopic 
two-fluid model are filtered to deduce closures for the corresponding filtered two-fluid model equations. We have found that, to a good approximation, the dimensionless filtered drag coefficient, particle phase pressure and particle phase (shear) viscosity can be treated as functions of only particle volume fraction and dimensionless filter size.

Previously, the filtered drag coefficient to describe the inter-phase interaction force in the filtered equations was studied for $\phi_{s}<0.30$ and shown to exhibit two distinct regimes. For $0.075<\phi_{s}<0.30$, it follows an effective Richardson-Zaki relationship and the effective R-Z exponent and apparent terminal velocity have an understandable physical interpretation in terms of interactions between particle clusters instead of the individual particles. At low particle volume fractions, the drag coefficient shows an anomalous behavior that is consistent with the formation of larger and denser clusters with increasing particle volume fraction. In both regimes, the filtered drag coefficient decreased with increasing filter size.

However, the closure relationships for the drag coefficient presented in our earlier work did not capture the regions of very low concentrations $\left(\phi_{s}<0.01\right)$ well and did not include high particle concentrations $\left(\phi_{s}>0.30\right)$ at all. In this study, we have investigated the region-averaged statistics in these regimes as well.

At extremely low particle volume fractions $\left(\sim 10^{-4}\right)$ all the curves corresponding to various filter lengths approach the same value indicating that the filtered drag coefficient is essentially independent of filter length in this regime. As the particle volume fraction increases, the filtered drag coefficient picks up a definite dependence on the filter size, manifesting a systematic decrease with increasing filter size. 
In the dense regions, we have seen the filtered drag coefficient cannot be captured with a Richardson-Zaki like model reasonably well. However, the dependence of the filtered closures on filter length is still clearly observed for $0.30<\phi_{s}<0.59$. At extremely high particle volume fractions (for $\phi_{s}>0.59$ ) all the curves corresponding to various filter lengths approach the same value indicating that the filtered closures are essentially independent of filter length in this regime. Furthermore, the filtered drag coefficient (for all filter sizes) at extremely high particle concentrations correspond to those used in the microscopic model ${ }^{30,40 .}$

We have sought an internally consistent model that combines all these regimes in a compact manner (as a function of particle volume fraction and filter length). To do this, we have analyzed the extended data obtained over a wide range of filter lengths and scaled them into correlations, thereby shedding some light on the asymptotic behavior at large filters. (See Table 2.)

We have presented several 3-D simulation results at the end to bring forth the differences between 2-D and 3-D closures flow and concluded that they are qualitatively similar. Therefore, we have modeled the 3-D filtered drag coefficient with constitutive relationships similar to those used for the 2-D filtered drag coefficient, see Table 3.

We have also closely studied the effect of filtering on the filtered particle phase pressure and shear viscosity, whose magnitudes are dictated by the velocity fluctuations associated with the very complicated inhomogeneous structures shown by the 2-D and 3-D microscopic twofluid model simulations, and have sought internally consistent constitutive relationships for these terms (as a function of particle volume fraction and filter length) for the entire particle phase 
volume fraction range. The filtering effect on these terms depends on the particle concentration in the filtering region.

The filtered particle phase pressure and shear viscosity increase with filter length for $\phi_{s}<0.59$, a direct consequence of the fact that the energy associated with the velocity fluctuations increases with filter length (as in single phase turbulence). At higher $\phi_{s}$ values, the sub-filter-scale velocity fluctuations disappear (the physical oscillations of clusters get restricted), and only the microscopic (kinetic) model particle phase pressure and shear viscosity remains. Consequently, the filtered particle phase viscosity becomes independent of the filtered size at extremely high $\phi_{s}$ values. The proposed 2-D and 3-D constitutive relationships capturing the particle volume fraction and filter size dependence are summarized in Table 2 and 3, respectively.

\section{Acknowledgments:}

This work was supported by the US Department of Energy (grants: CDE-FC2600NT40971 and DE-PS26-05NT42472-11) and the ExxonMobil Research \& Engineering Company. Igci acknowledges summer training on MFIX at the National Energy Technology Laboratory, Morgantown, WV.

\section{Nomenclature}

$a \quad$ Weighting function (for filtered drag coefficient modeling)

$b \quad$ Exponent of the weighting function for the dilute region (for filtered drag coefficient modeling)

$d_{p} \quad$ Particle diameter $(\mathrm{m})$

$e_{p} \quad$ Coefficient of restitution for particle-particle collisions 
factor $_{\text {dense }} \quad$ Weighting function for the dense region (for filtered drag coefficient modeling)

factor $_{\text {dilute }} \quad$ Weighting function for the dilute region (for filtered drag coefficient modeling)

Factor $_{p s} \quad$ Coefficient used in modeling the filtered particle phase pressure

Factor $_{\mu \mathrm{s}} \quad$ Coefficient used in modeling the filtered particle phase shear viscosity

$\mathrm{Fr}_{f} \quad$ Froude number based on filter length $=\mathrm{v}_{\mathrm{t}}^{2} / g \Delta_{f}$

$\mathrm{Fr}_{g} \quad$ Froude number based on grid length $=\mathrm{v}_{\mathrm{t}}^{2} / g \Delta_{g}$

Function $_{\text {total }} \quad$ An arithmetic weighted sum of the functions capturing the R-Z like region and that for the microscopic model

Function $_{\text {microscopic }}$ Natural logarithmic value of the microscopic drag coefficient

Function $_{\text {asymptotic }}$ The asymptotic value of R-Z like region

$g, \mathbf{g} \quad$ Acceleration due to gravity $\left(\mathrm{m} / \mathrm{s}^{2}\right)$

$H_{D} \quad$ The heterogeneity index

$m, n \quad$ Exponential coefficients used for scaling the filtered drag coefficient

$N_{R Z, a p p} \quad$ Apparent Richardson-Zaki exponent

$p, q \quad$ Exponents used for scaling the filtered particle stress terms

$\overline{p_{s, \text { filtered }}} \quad$ Filtered particle phase pressure $\left(\mathrm{kg} / \mathrm{m} . \mathrm{s}^{2}\right)$

$\overline{p_{s, \text { filtered }, d}} \quad \overline{p_{s, \text { filtered }, d}}$ made dimensionless; $\overline{p_{s, \text { fitered }, d}}=\overline{p_{s, \text { filtered }}} / \rho_{s} v_{t}^{2}$

$\overline{p_{s, k i n e t i c}} \quad$ Filtered value of particle phase pressures in the kinetic theory model $\left(\mathrm{kg} / \mathrm{m} . \mathrm{s}^{2}\right)$

$\mathrm{v}_{\mathrm{t}} \quad$ Terminal settling velocity $(\mathrm{m} / \mathrm{s})$

$\mathbf{v} \quad$ Particle phase velocity in the microscopic two-fluid model $(\mathrm{m} / \mathrm{s})$

$\overline{\mathbf{v}} \quad$ Filtered particle phase velocity $(\mathrm{m} / \mathrm{s})$ 

$\mathbf{v}^{\prime}$
Fluctuations in particle phase velocity $(\mathrm{m} / \mathrm{s})$
$\mathbf{x}, \mathbf{y}$
Position vectors (m)
$V_{\text {slip }} \quad$ Vertical slip velocity $(\mathrm{m} / \mathrm{s})$
$V_{t, a p p} \quad$ Dimensionless apparent terminal velocity

\section{Greek Symbols}

$\overline{\beta_{\text {filtered }}}$

$\overline{\beta_{\text {asymptotic }}}$

$\overline{\beta_{\text {microscopic }}}$

$\overline{\beta_{\text {fitered }, d}}$

$\phi_{s}, \phi_{g}$

$\phi_{\mathrm{s}, \max }$

$\overline{\phi_{s}}, \overline{\phi_{g}}$

$\rho_{s}, \rho_{g}$

$\Delta_{f}$

$\Delta_{g}$

$\underline{\underline{\sigma_{s}}}$

$\mu_{g}$
Filtered drag coefficient $\left(\mathrm{kg} / \mathrm{m}^{3} . \mathrm{s}\right)$

Asymptotic value of the filtered drag coefficient $\left(\mathrm{kg} / \mathrm{m}^{3} . \mathrm{s}\right)$

Microscopic filtered drag coefficient $\left(\mathrm{kg} / \mathrm{m}^{3} . \mathrm{s}\right)$

Dimensionless filtered drag coefficient $=\overline{\beta_{\text {filtered }}} \mathrm{V}_{\mathrm{t}} / \rho_{\mathrm{s}} g$

Particle and gas phase volume fractions, respectively

Maximum particle volume fraction

Filtered particle and gas phase volume fractions, respectively

Particle and gas densities, respectively $\left(\mathrm{kg} / \mathrm{m}^{3}\right)$

Filter length (m)

Grid length (m)

Particle phase stress tensor in the kinetic theory model $\left(\mathrm{kg} / \mathrm{m} . \mathrm{s}^{2}\right)$

Gas phase viscosity $(\mathrm{kg} / \mathrm{m} . \mathrm{s})$ 
$\mu_{s}$ Shear viscosity of the particle phase appearing in the kinetic theory model $(\mathrm{kg} / \mathrm{m} . \mathrm{s})$

$\overline{\mu_{s, \text { filtered }}}$ Shear viscosity of the particle phase appearing in the filtered two-fluid $\operatorname{model}(\mathrm{kg} / \mathrm{m} . \mathrm{s})$

$\overline{\mu_{s, \text { filtered,d }}}$

$\overline{\mu_{s, \text { filtered }}}$ made dimensionless; $\overline{\mu_{s, \text { filtered }, d}}=\overline{\mu_{s, \text { filtered }}} g / \rho_{\mathrm{s}} \mathrm{v}_{\mathrm{t}}^{3}$.

\section{References:}

See the references section of the main text of this report for the references cited in Appendix C. 
Table 2: Sample physical properties of the gas and particles

\begin{tabular}{lll}
\hline$d_{p}$ & Particle diameter & $7.5 \times 10^{-6} \mathrm{~m}$ \\
$\rho_{\mathrm{s}}$ & Particle density & $1500 \mathrm{~kg} / \mathrm{m}^{3}$ \\
$\rho_{\mathrm{g}}$ & Gas density & $1.3 \mathrm{~kg} / \mathrm{m}^{3}$ \\
$\mu_{\mathrm{g}}$ & Gas viscosity & $1.8 \times 10^{-5} \mathrm{~kg} / \mathrm{m} \cdot \mathrm{s}$ \\
$e_{p}$ & Coefficient of restitution & 0.9 \\
$\mathrm{~g}$ & Gravitational acceleration & $9.80665 \mathrm{~m} / \mathrm{s}^{2}$ \\
$\mathrm{v}_{\mathrm{t}}$ & Terminal settling velocity & $0.2184 \mathrm{~m} / \mathrm{s}$ \\
$\frac{\mathrm{v}_{\mathrm{t}}^{2}}{\mathrm{~g}}$ & Characteristic length & $0.00487 \mathrm{~m}$ \\
$\frac{\mathrm{v}_{\mathrm{t}}}{\mathrm{g}}$ & Characteristic time & $0.0223 \mathrm{~s}$ \\
$\rho_{\mathrm{s}} \mathrm{v}_{\mathrm{t}}^{2}$ & Characteristic stress & $71.55 \mathrm{~kg} / \mathrm{m} \cdot \mathrm{s}^{2}$ \\
\hline
\end{tabular}

Table 2: 2-D Filtered closures

See Table 2 in the main text of this report.

Table 3: 3-D Filtered closures

See Table 3 in the main text of this report. 
FIGURES and CAPTIONS:

a)

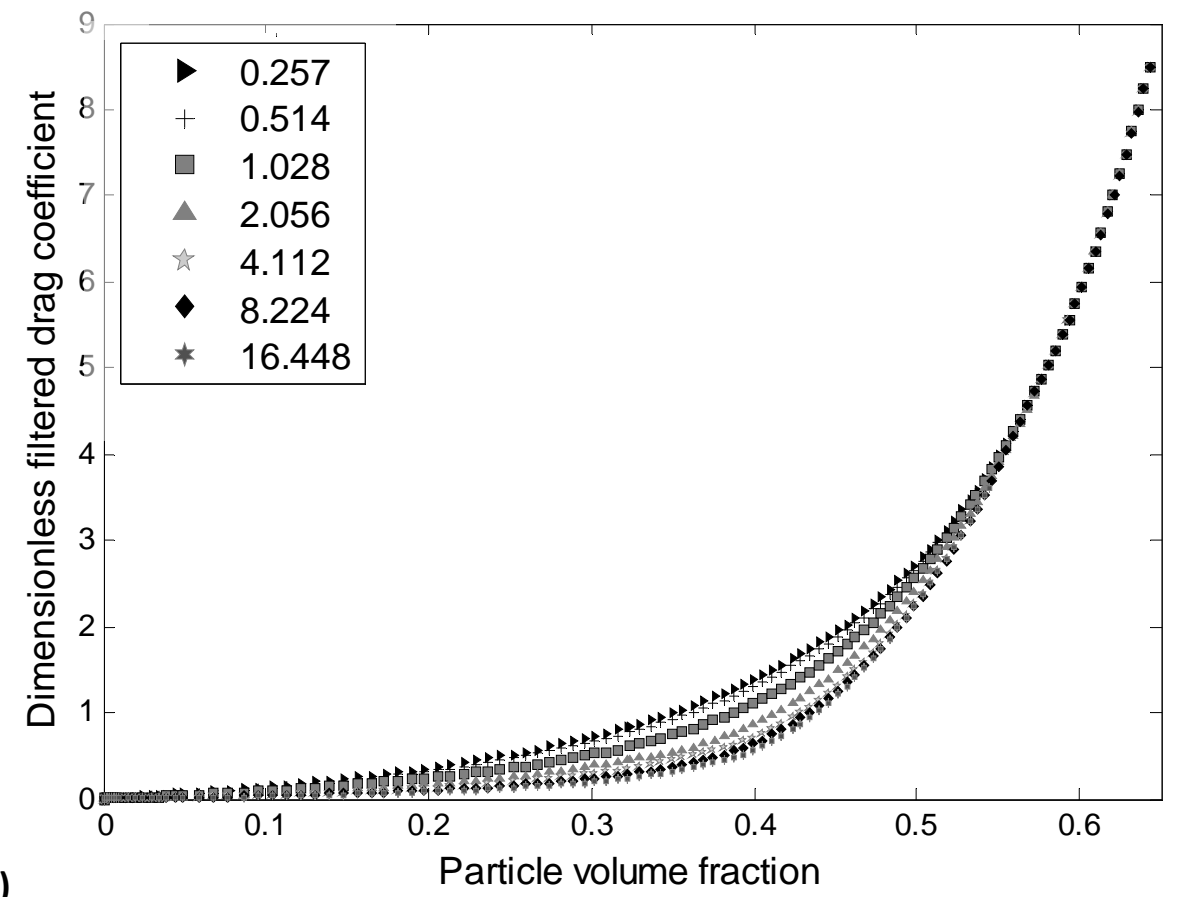

b)

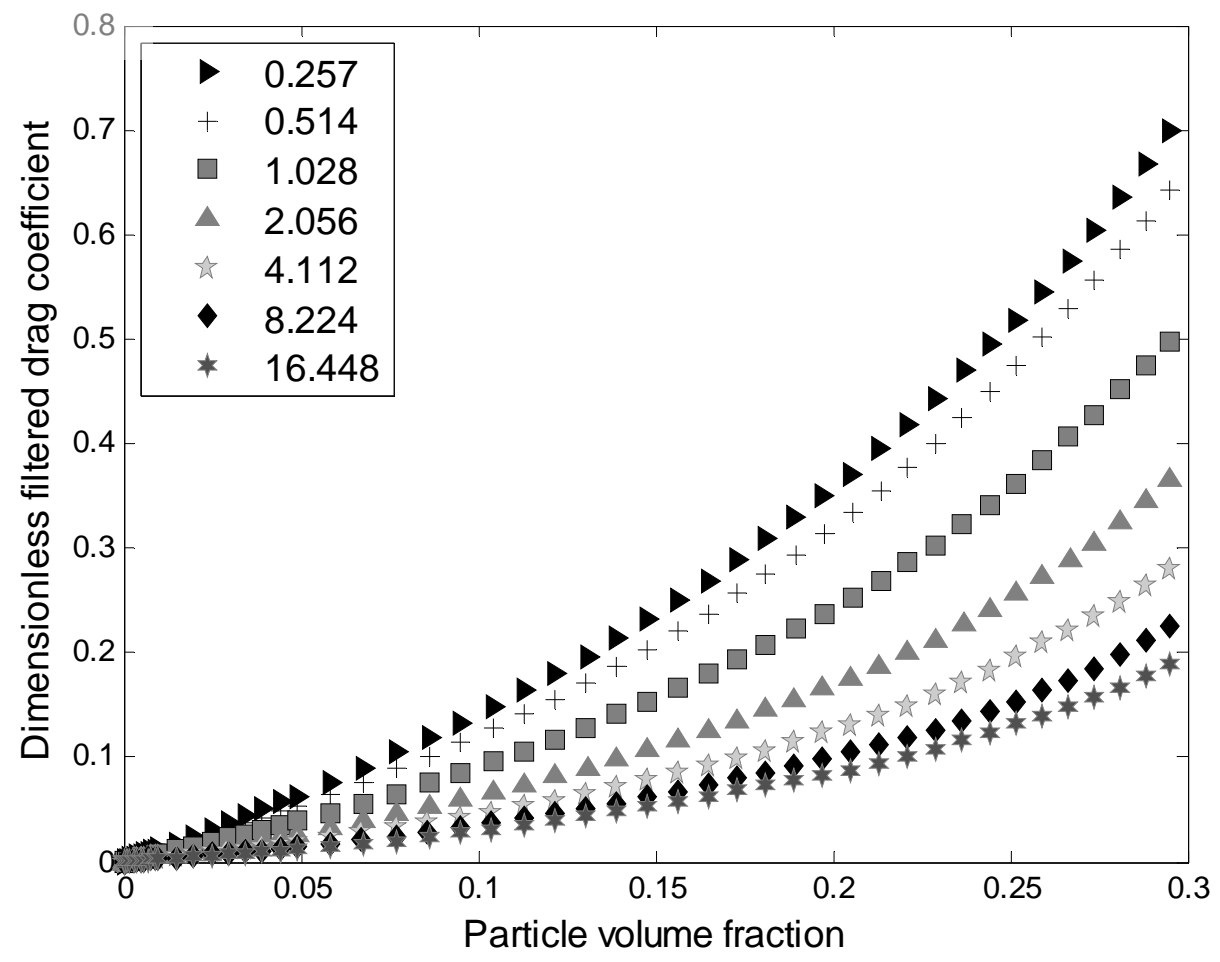




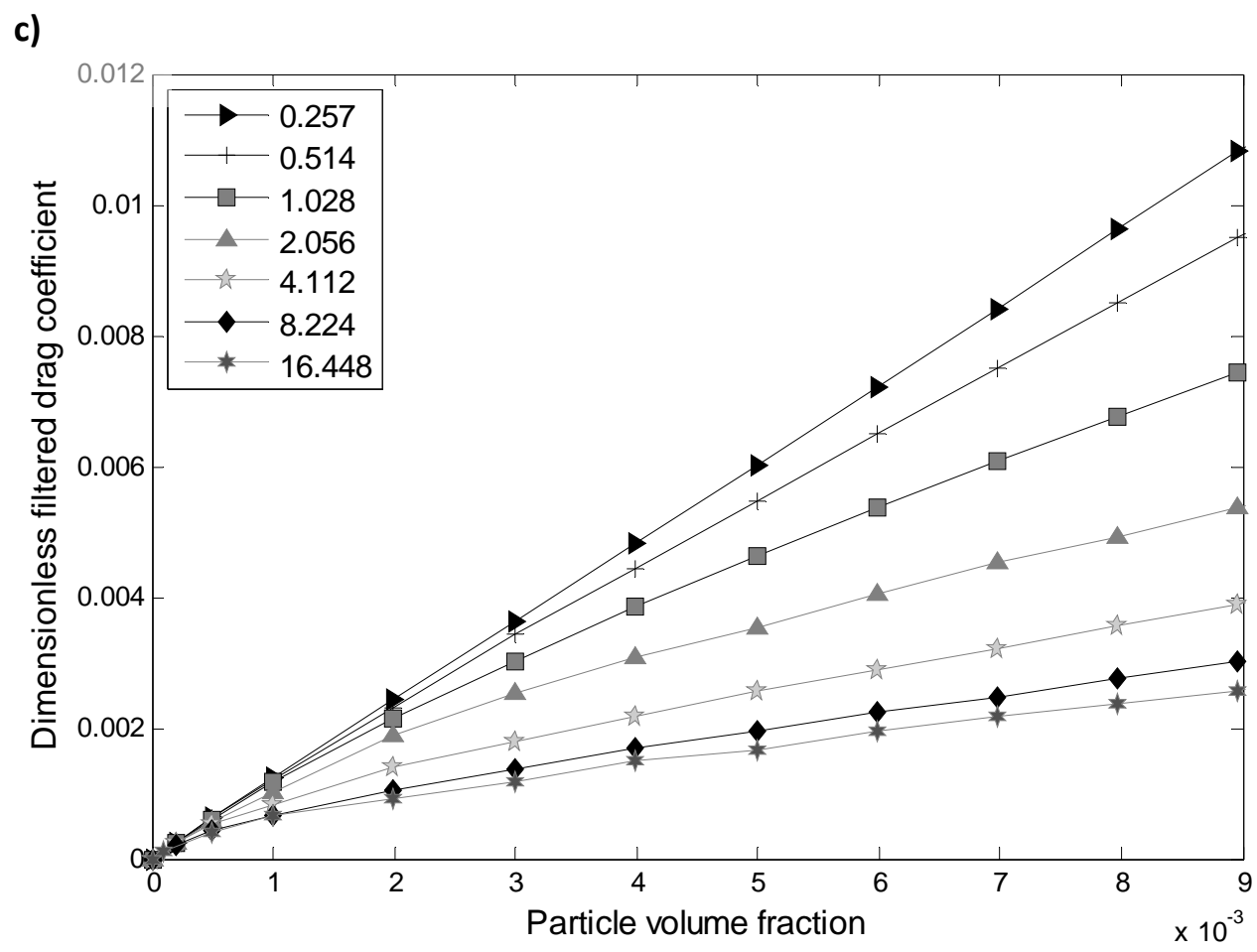

Figure 1: The variation of the dimensionless filtered drag coefficient with particle volume fraction for various filter lengths (listed in the legend in dimensionless units) is shown. Simulations were performed in a square periodic domain of size $131.584 \times 131.584$ dimensionless units and using 512 × 512 grid points. Data used for filtering were generated by running simulations for domain-average particle volume fractions of $.01,0.02,0.05,0.10,0.15,0.20,0.25,30,0.35,0.40,0.45,0.50,0.55$ and 0.58 . The dimensionless filter lengths are shown in the legend. (a) The entire particle volume fraction range ( $0 \leq \phi_{s}<0.65$ ); (b) for $0 \leq \phi_{s}<0.30$ (to show the filtering effect more clearly in the regions of moderate particle concentration); (c) for the dilute volume fraction region $\left(0 \leq \phi_{s}<0.009\right)$. 
a)

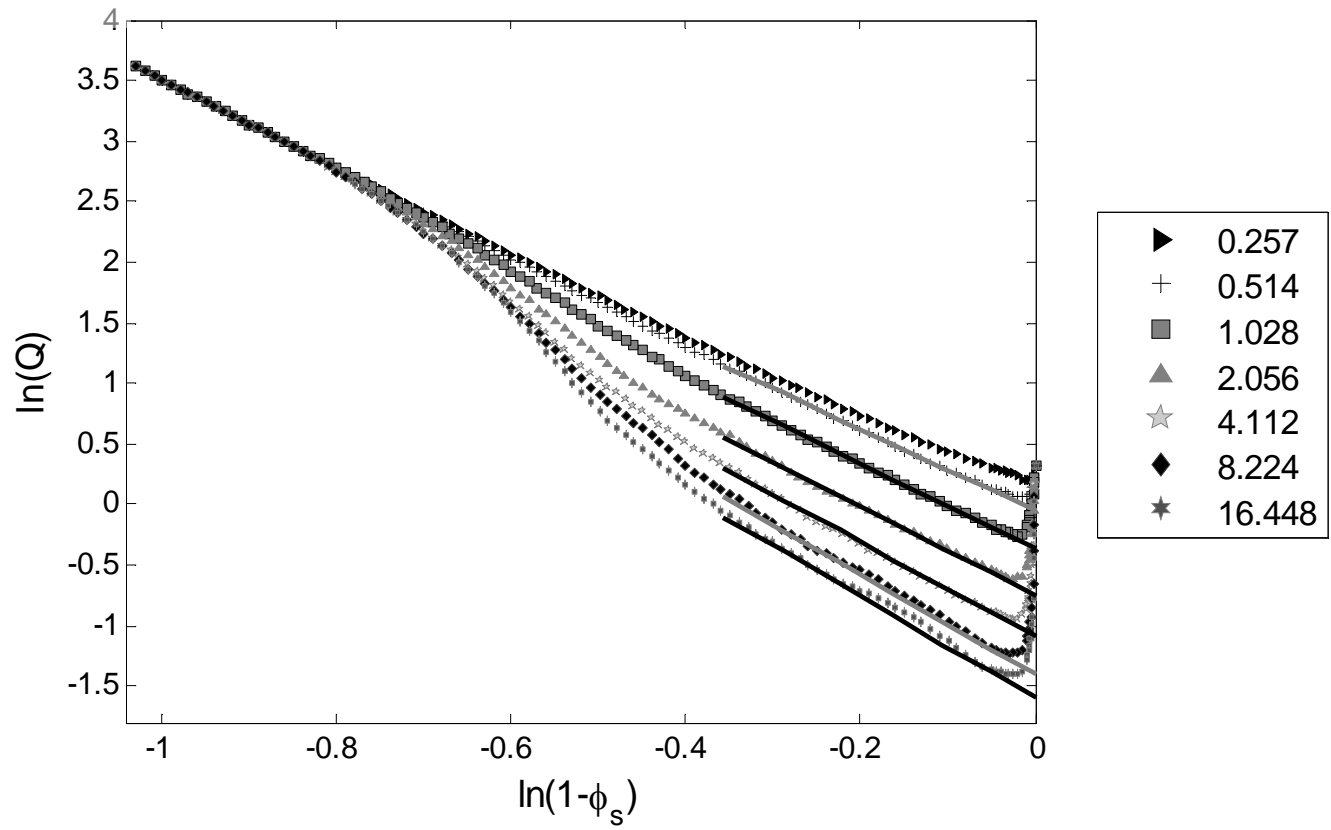

b)

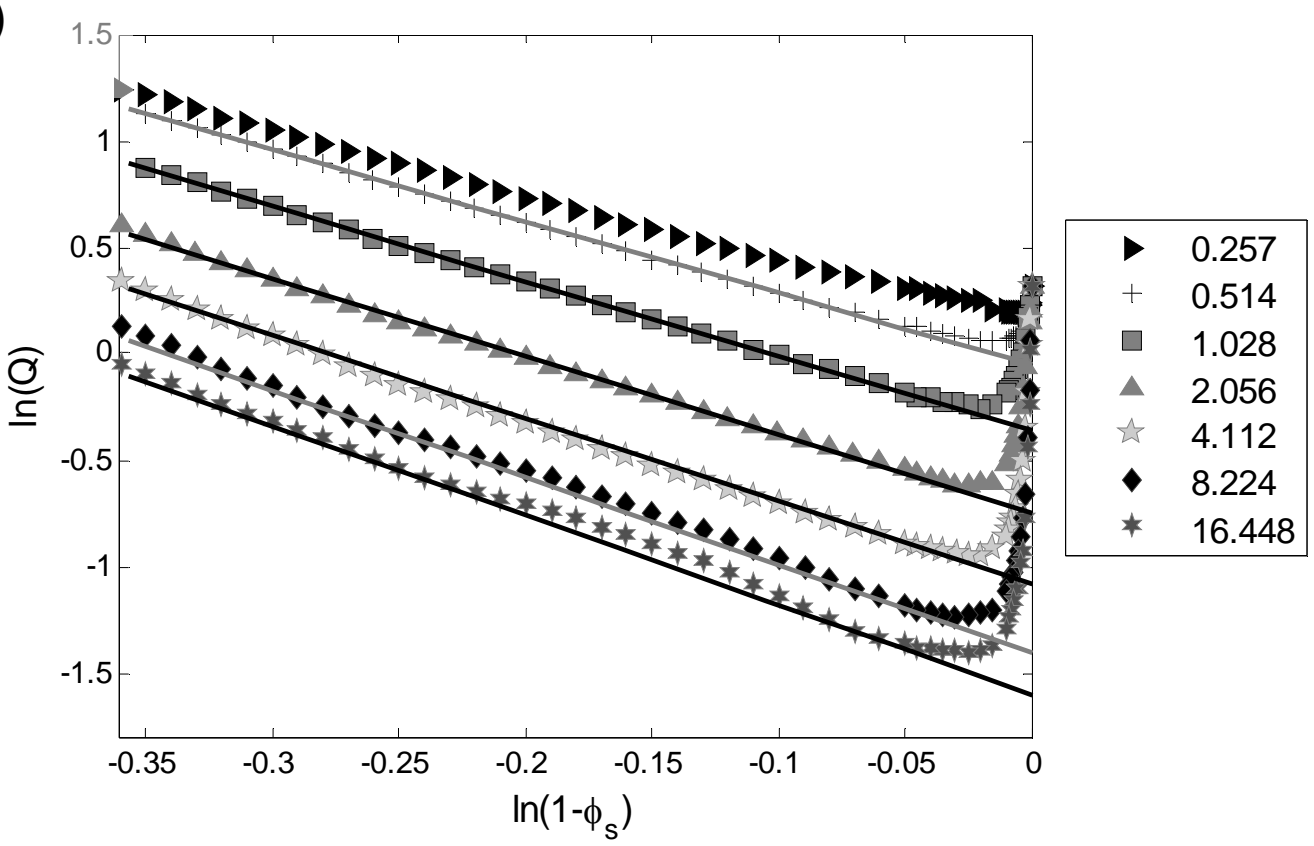

Figure 2: The results shown earlier in Figures $1(a-b)$ are plotted on a natural logarithmic scale. Here $Q=\frac{\overline{\beta_{\text {filtered }, d}}}{\phi_{s}\left(1-\phi_{s}\right)}$, where $\overline{\beta_{\text {fitered }, d}}$ is the dimensionless filtered drag coefficient, $\phi_{s}$ is particle phase volume fraction, The dimensionless filter lengths are shown in the legend. (a) For $0 \leq \phi_{s}<0.65$ (the entire particle volume fraction range); (b) for $0 \leq \phi_{s}<0.30$ (to show the filtering effect more clearly in the regions of moderate particle concentration). 
a)

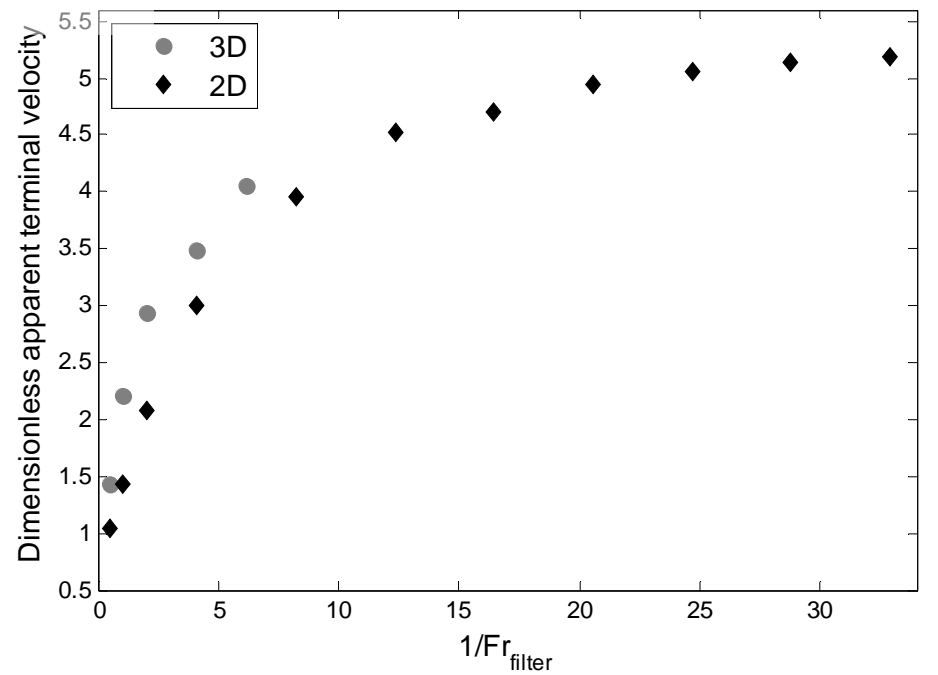

b)
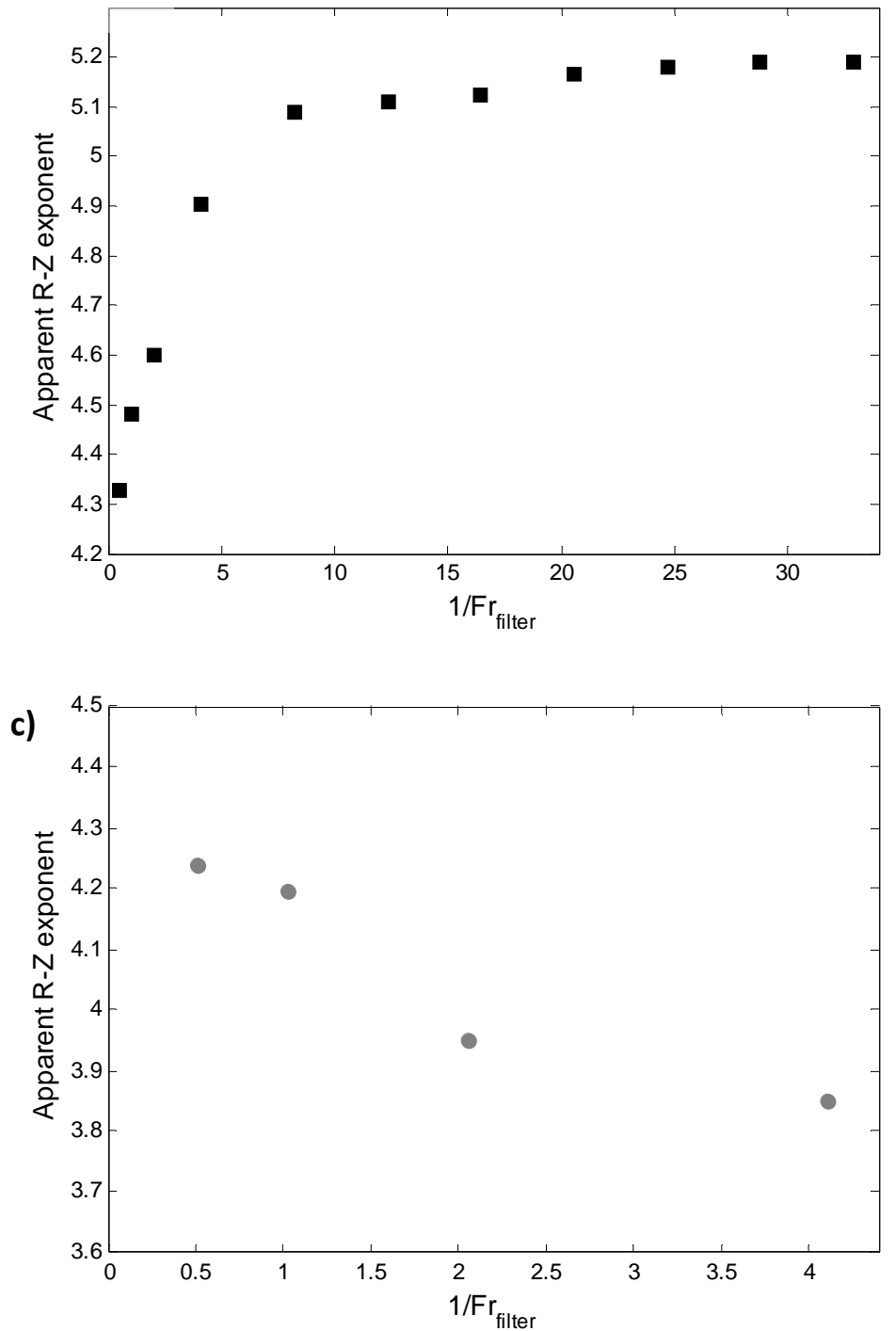
Figure 3: (a) Dimensionless apparent terminal velocity $\left(V_{t, a p p}\right)$ for various dimensionless filter lengths, extracted from results in Figure 2 (a-b) (2D) for the particle volume fraction range $0.05<\phi_{s}<0.30$ and those extracted from results in Figure $2(\mathrm{a}-\mathrm{b})(3 \mathrm{D})$ for the range $0.075<\phi_{s}<0.25$. The solid lines in Figure 2 (a-b) for a filter length of 2.056 is based on $V_{t, a p p}$ shown here and the apparent Richardson-Zaki exponent ( $N_{R Z, a p p}$ ) in Figure 3 (b). (b) $N_{R Z, a p p}$ for various dimensionless filter lengths, extracted from results in Figure 2(a-b) (2D) for the range $0.05<\phi_{s}<0.30$. (c) $N_{R Z, a p p}$ for various dimensionless filter lengths, extracted from results in Figure 2 (a-b) (3D) for the range $0.075<\phi_{s}<0.25$. 


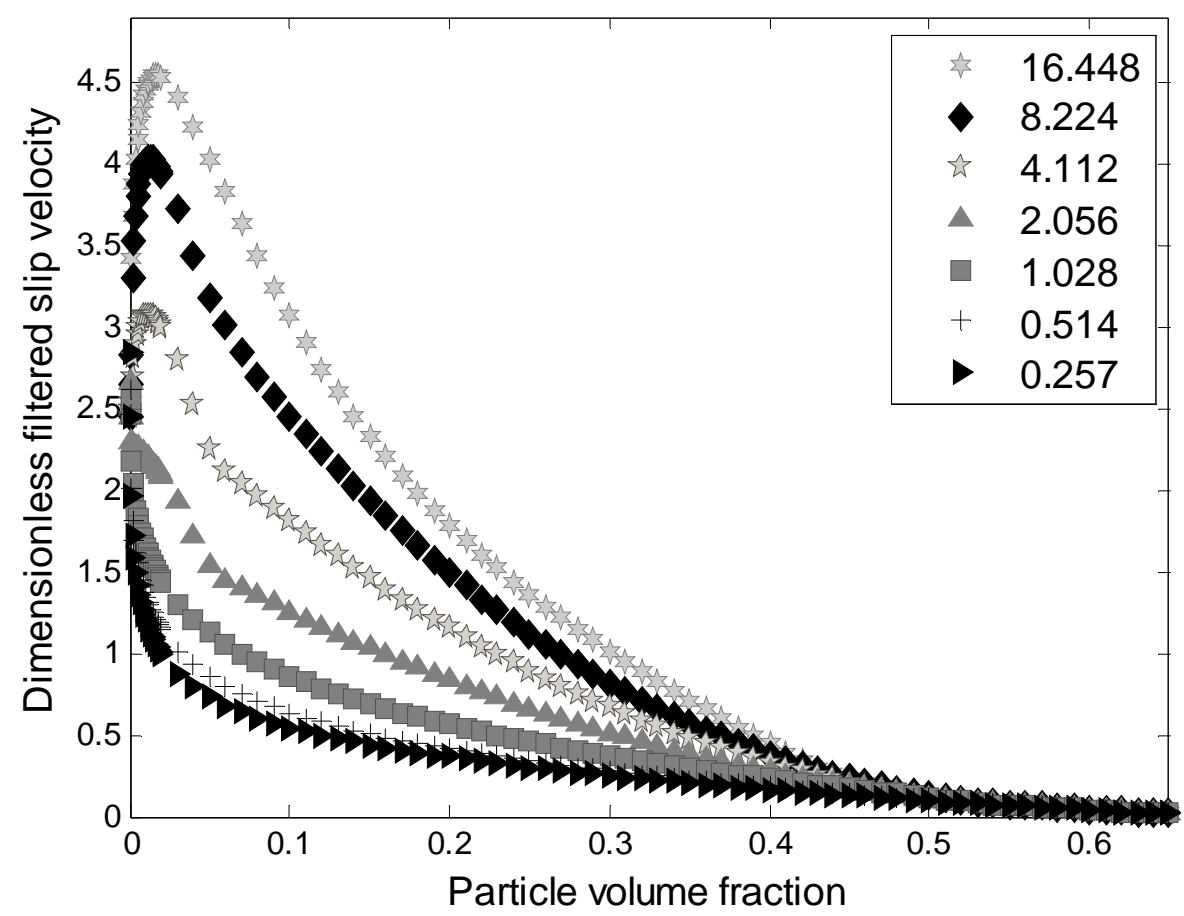

Figure 4: The variation of dimensionless filtered slip velocity with filtered particle volume fraction is shown for various dimensionless filter lengths shown in the legend. These results were generated from the same set of simulation data that led to Figure 1(a-c). 


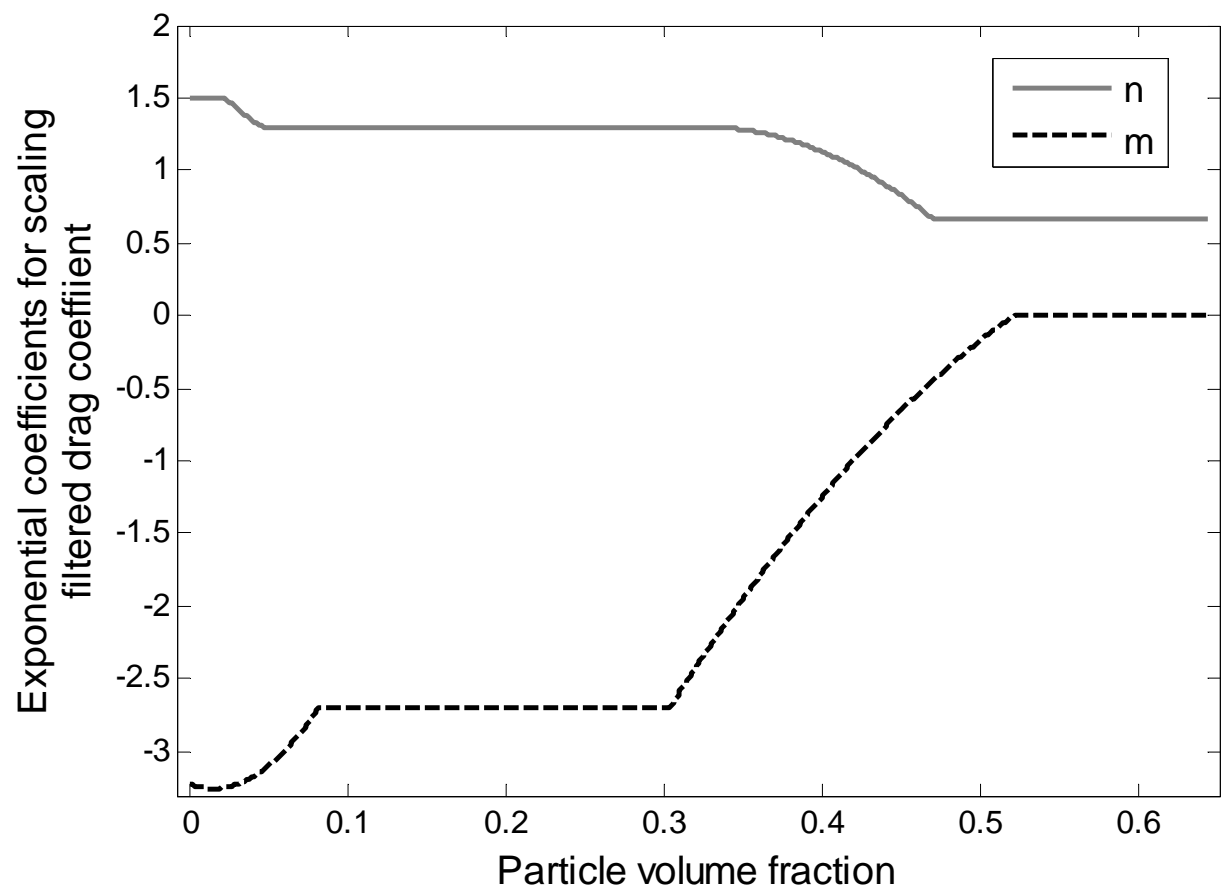

Figure 5: The variation of exponential coefficients " $\mathrm{m}$ " and " $\mathrm{n}$ " for scaling filtered drag coefficient with particle volume fraction. For $F r_{f}^{-1} \geq 4.112$, the filtered drag coefficient was found to vary roughly as $\left(F r_{f}\right)^{m\left(F r_{f}\right)^{n}}$. Here, $F r_{f}^{-1}=g \Delta_{f} / v_{t}^{2}$. See the main text for the details. 


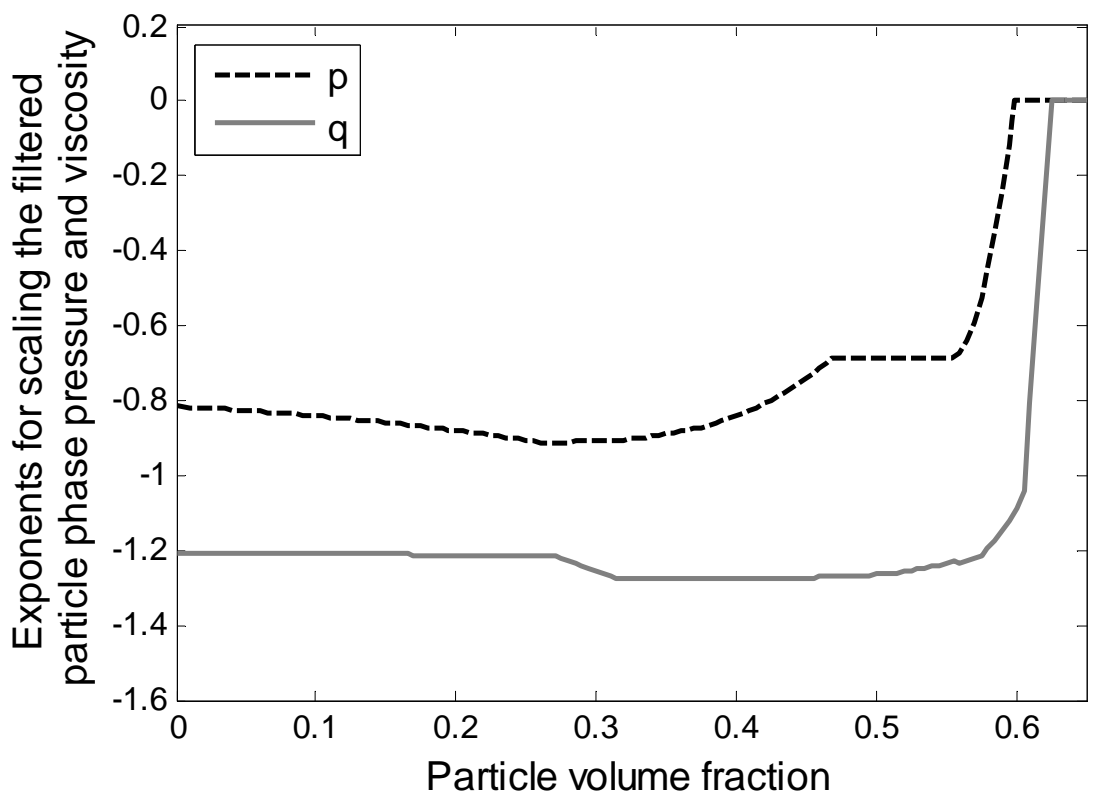

Figure 6: The effect of particle volume fraction on exponents " $p$ " and " $q$ " for scaling the 2-D filtered particle phase stress terms. For $\mathrm{Fr}_{f}^{-1} \geq 4.112$, the filtered particle phase pressure and shear viscosity were found to vary roughly as $\left(F r_{f}\right)^{p}$ and $\left(F r_{f}\right)^{q}$, respectively. $F r_{f}^{-1}=g \Delta_{f} / v_{t}^{2}$. See the main text for the details. 
a)

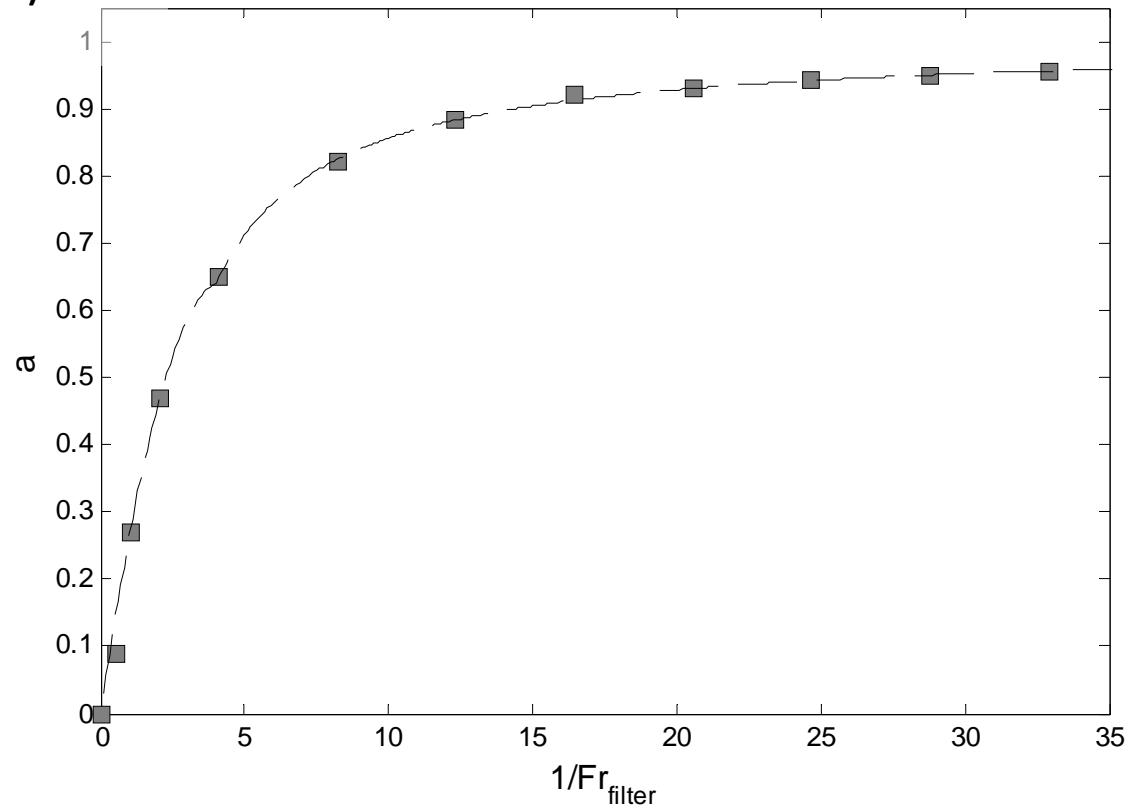

b)

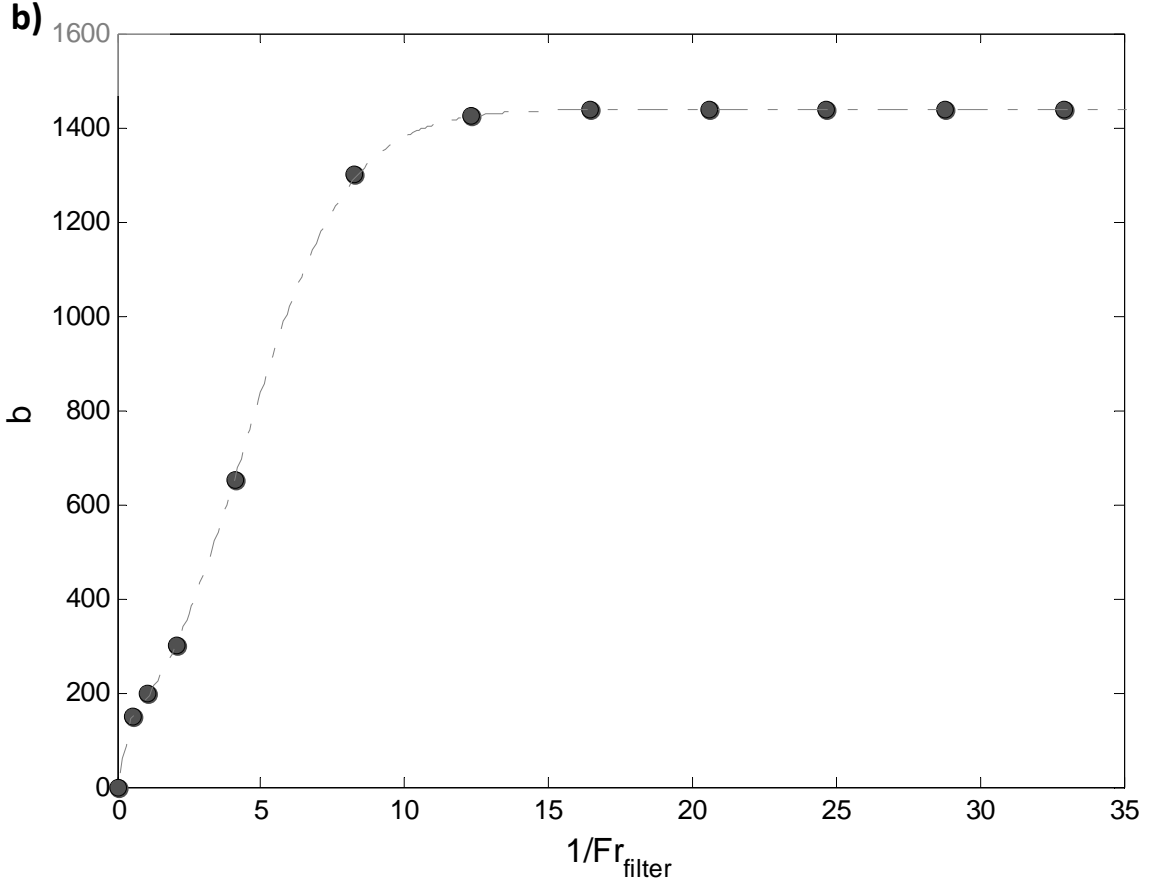

Figure 7: (a) The variation of the 2-D weighting function "a" (see Equation 3 in the main text) with dimensionless filter size. "a" can be modeled with Equation 5 in the main text (represented by a dashed line). (b) The variation of the exponent " $b$ " for the dilute region (see Equation 10 in the main text) extracted from the results in Figure 2 (a-b), as a function of dimensionless filter length. The dash-dotted line represents the function fitting for the data; see Equation 11 in the main text. 
a)
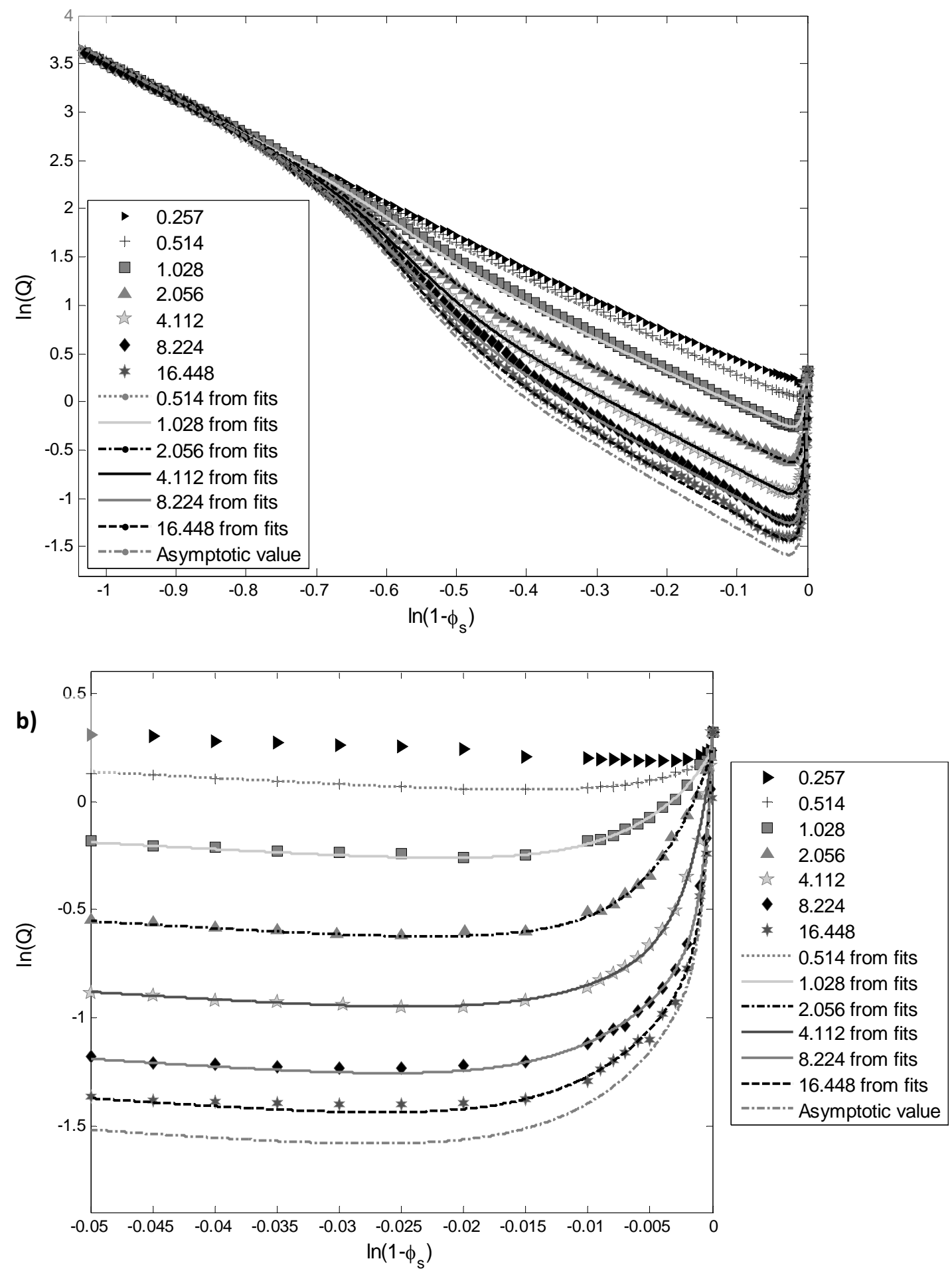

Figure 8: The results $(\ln (\mathrm{Q}))$ shown in Figure 2(a-b) are re-plotted to present a comparison of the simulation data with the constitutive model shown in Equation 9 (in the main text) for (a) $0 \leq \phi_{s}<0.65$ and (b) $\phi_{s}<0.05$. The bottom line indicates asymptotic value of $\ln (\mathrm{Q})$ for large filters, which was obtained from scaling studies. 
a)

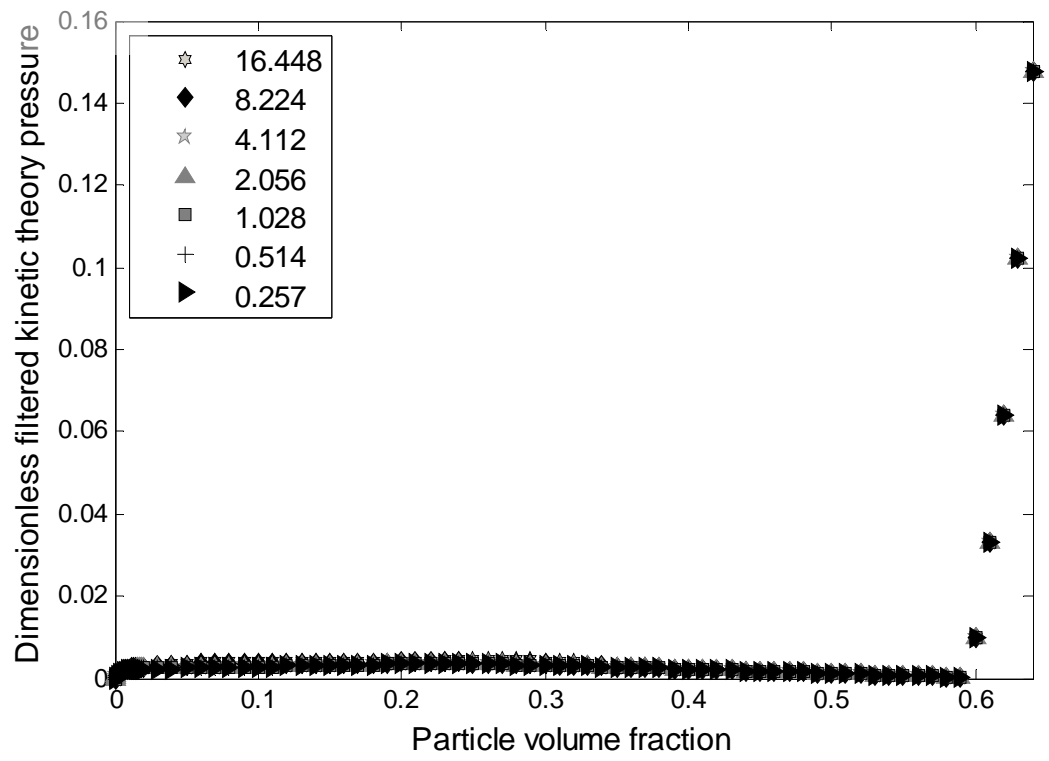

b)

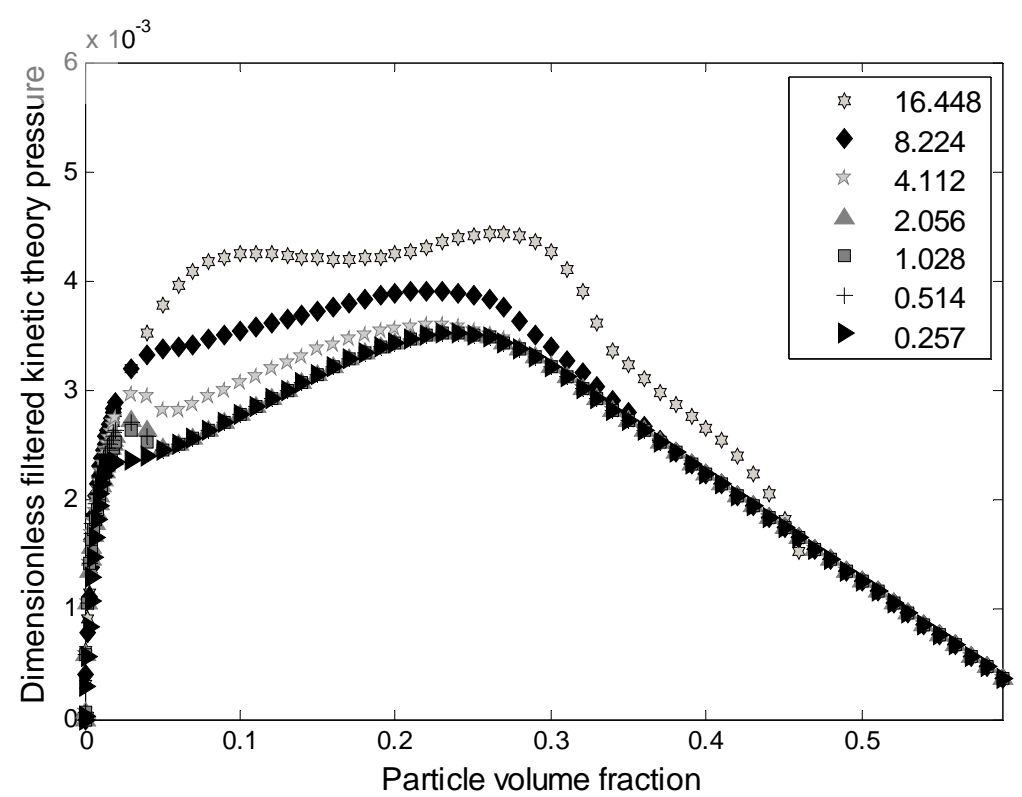

Figure 9: The variation of the dimensionless filtered kinetic theory pressure with particle volume fraction is presented for various dimensionless filter lengths. The results were extracted from simulations mentioned in the caption for Figure 1(a-c). The dimensionless filter lengths are shown in the legend. (a) For $0 \leq \phi_{s}<0.65$; (b) for $0 \leq \phi_{s}<0.59$ (to show the filtering effect more clearly). 
a)

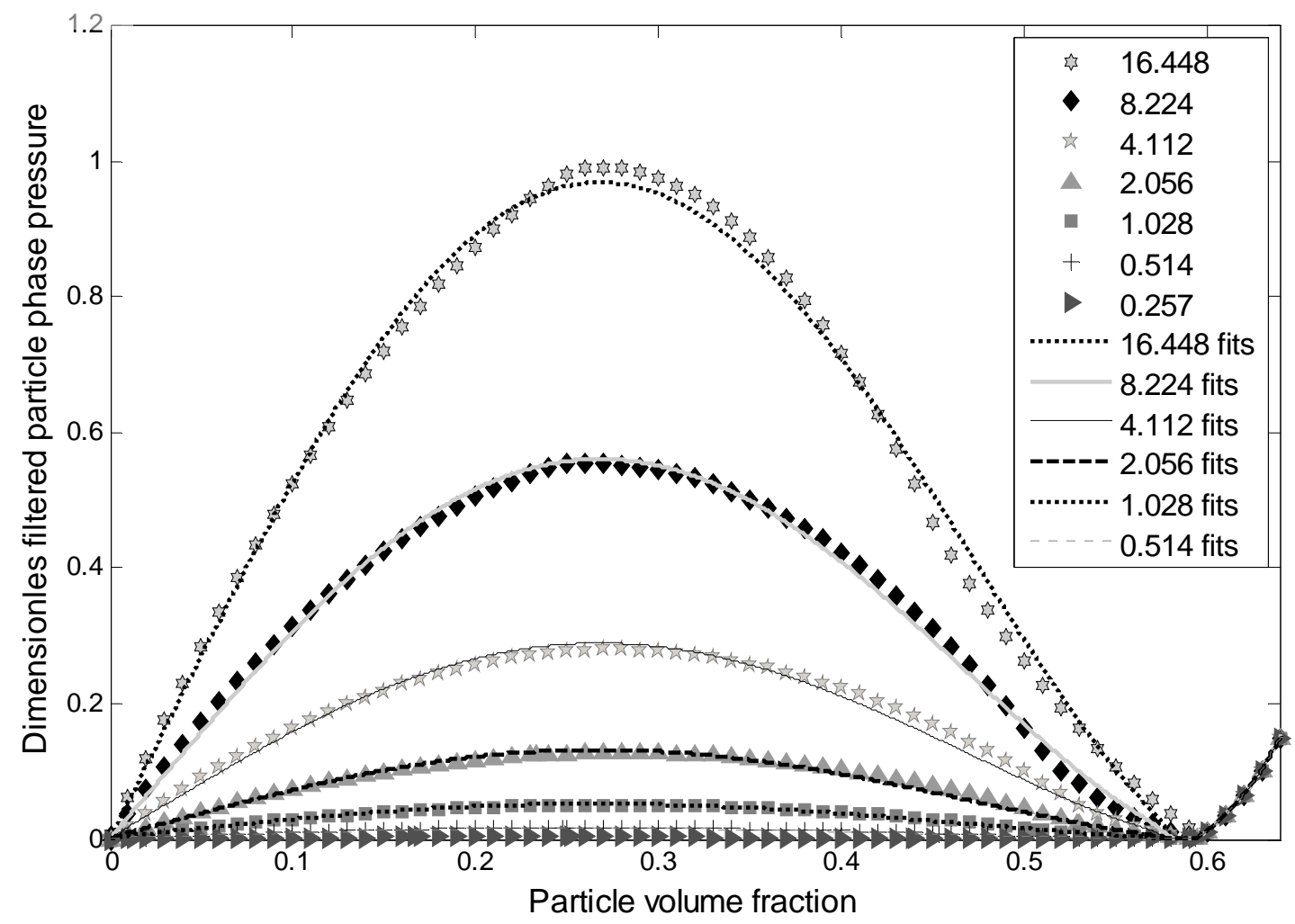

b)

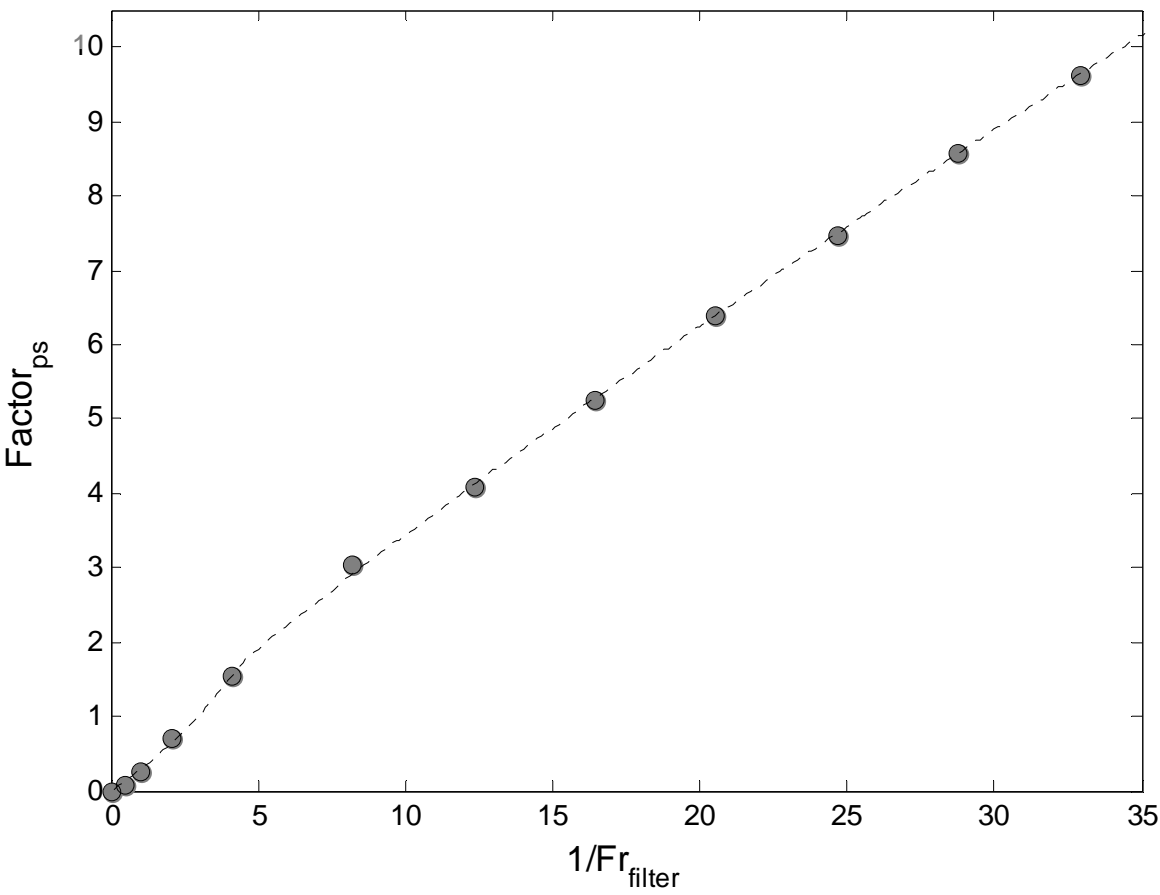


Figure 10: (a) The effect of particle phase volume fraction on the dimensionless filtered particle phase pressure for the dimensionless filter lengths shown in the figure legend. The results were extracted from simulations mentioned in the caption for Figure 1(a-c). The figure also includes the constitutive relationships (as a function of particle volume fraction and filter size) given in Equation 14. (b) The coefficient "Factor ${ }_{\mathrm{ps}}$ " in Equation 14 is plotted against the dimensionless filter length. "Factor ${ }_{\mathrm{ps}}$ " can be modeled as shown in Equation 16 (represented by the dashed line). 
a)

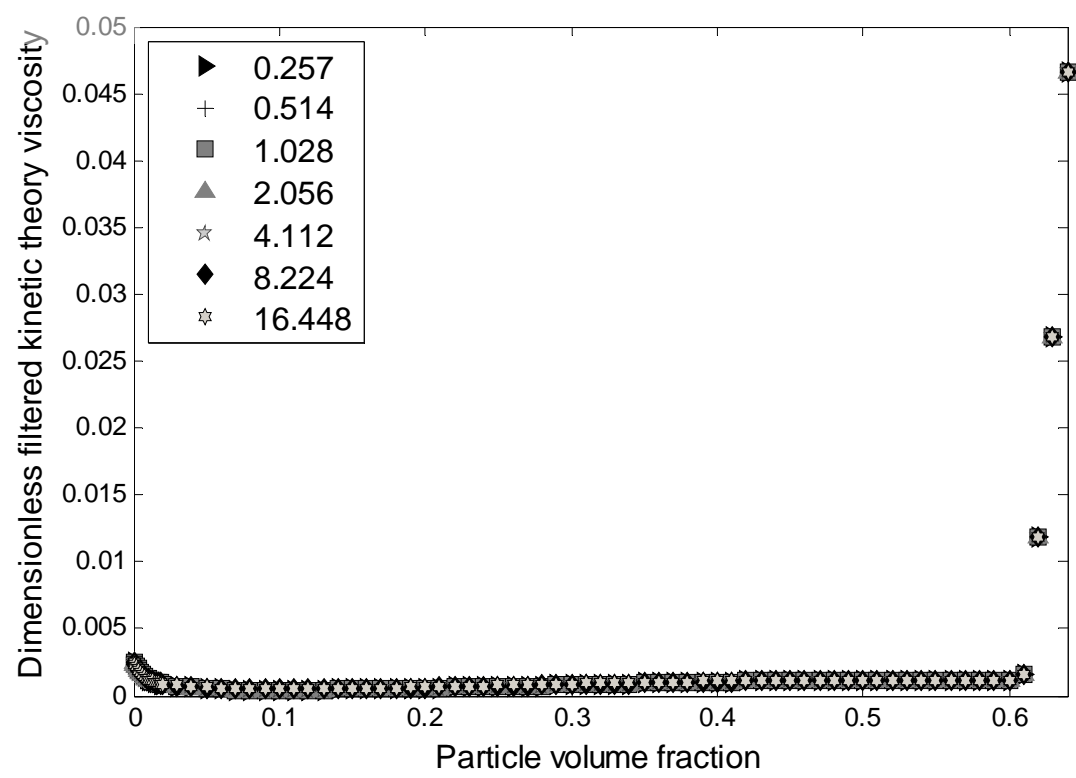

b)

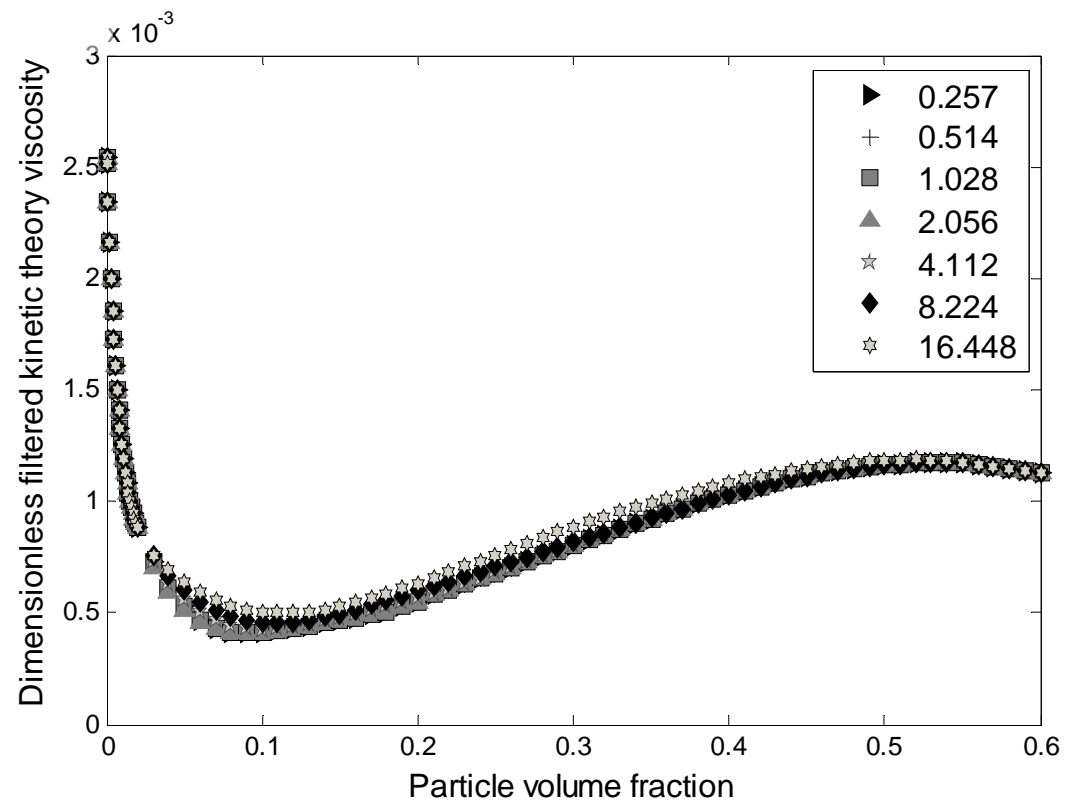

Figure 11: The variation of the dimensionless filtered kinetic theory viscosity with particle volume fraction is presented for the seven dimensionless filter lengths shown in the figure legend. The results were extracted from simulations mentioned in the caption for Figure 1(a-c). (a) For $0 \leq \phi_{s}<0.65$; (b) for $0 \leq \phi_{s}<0.59$ (to show the filtering effect more clearly). 


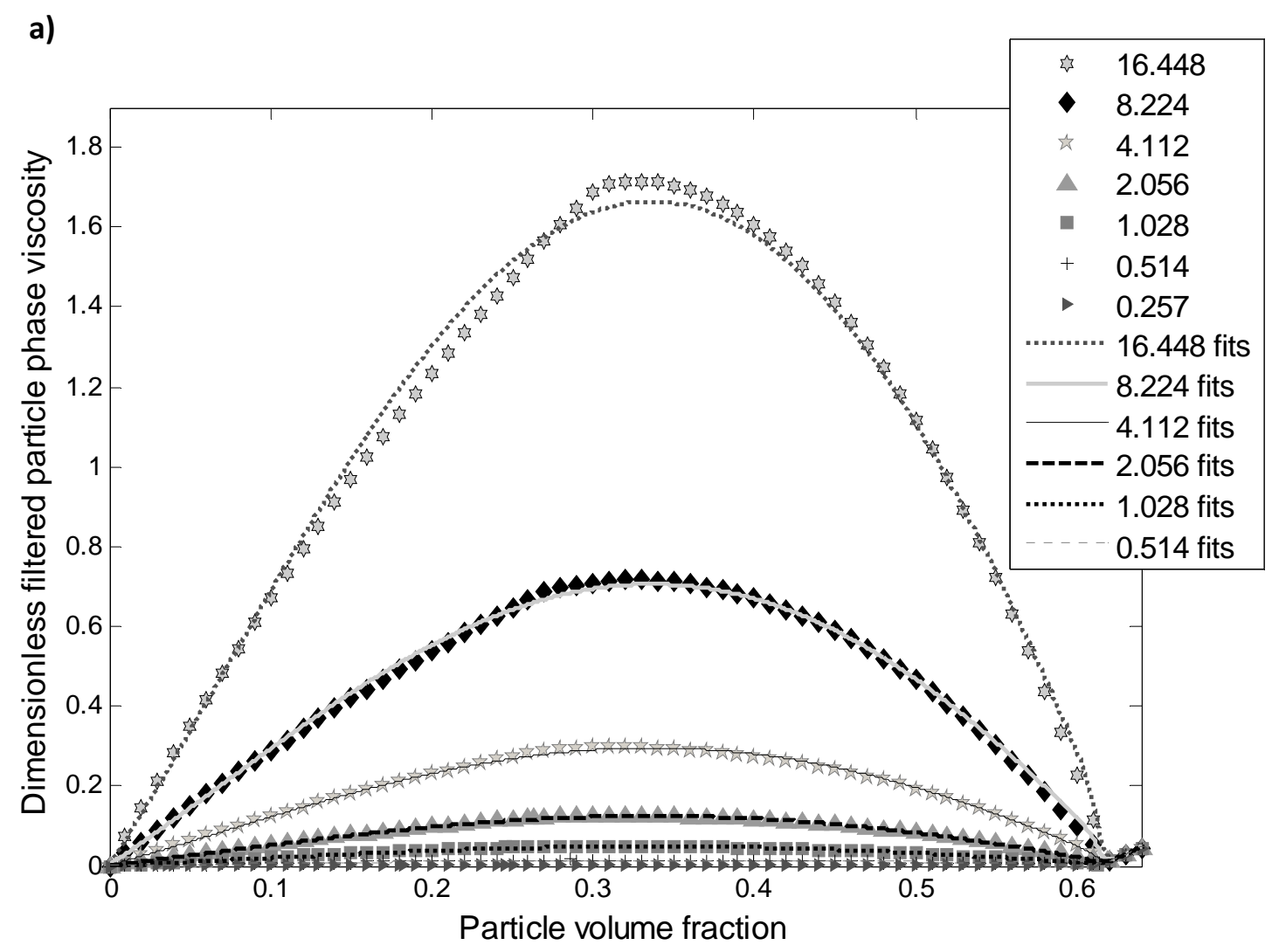

b)

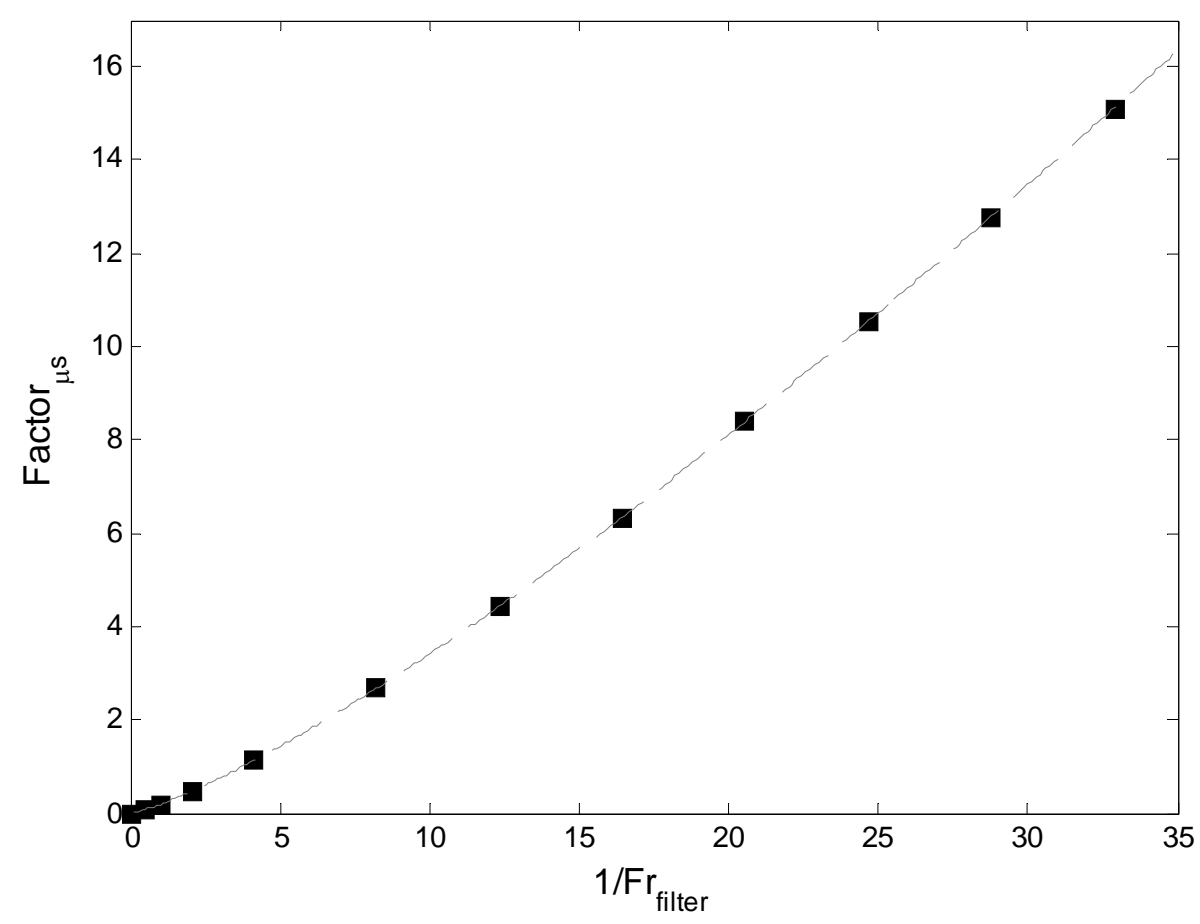


Figure 12: (a) The variation of the dimensionless (total) filtered particle phase shear viscosity with particle volume fraction is presented for seven different dimensionless filter lengths. The results were extracted from simulations mentioned in the caption for Figure 1(a-c). The dimensionless filter lengths are shown in the legend. The figure also includes the constitutive relationship (as a function of particle volume fraction and filter size) given in Equation T2.15 (in Table 2). (b) The coefficient "Factor ${ }_{\mu \text { s" in }}$ Equation T2.15 is plotted against the dimensionless filter length. "Factor ${ }_{\mu \mathrm{s}}$ " can be modeled as shown in Equation T2.17 (represented by the dashed line). 
a)

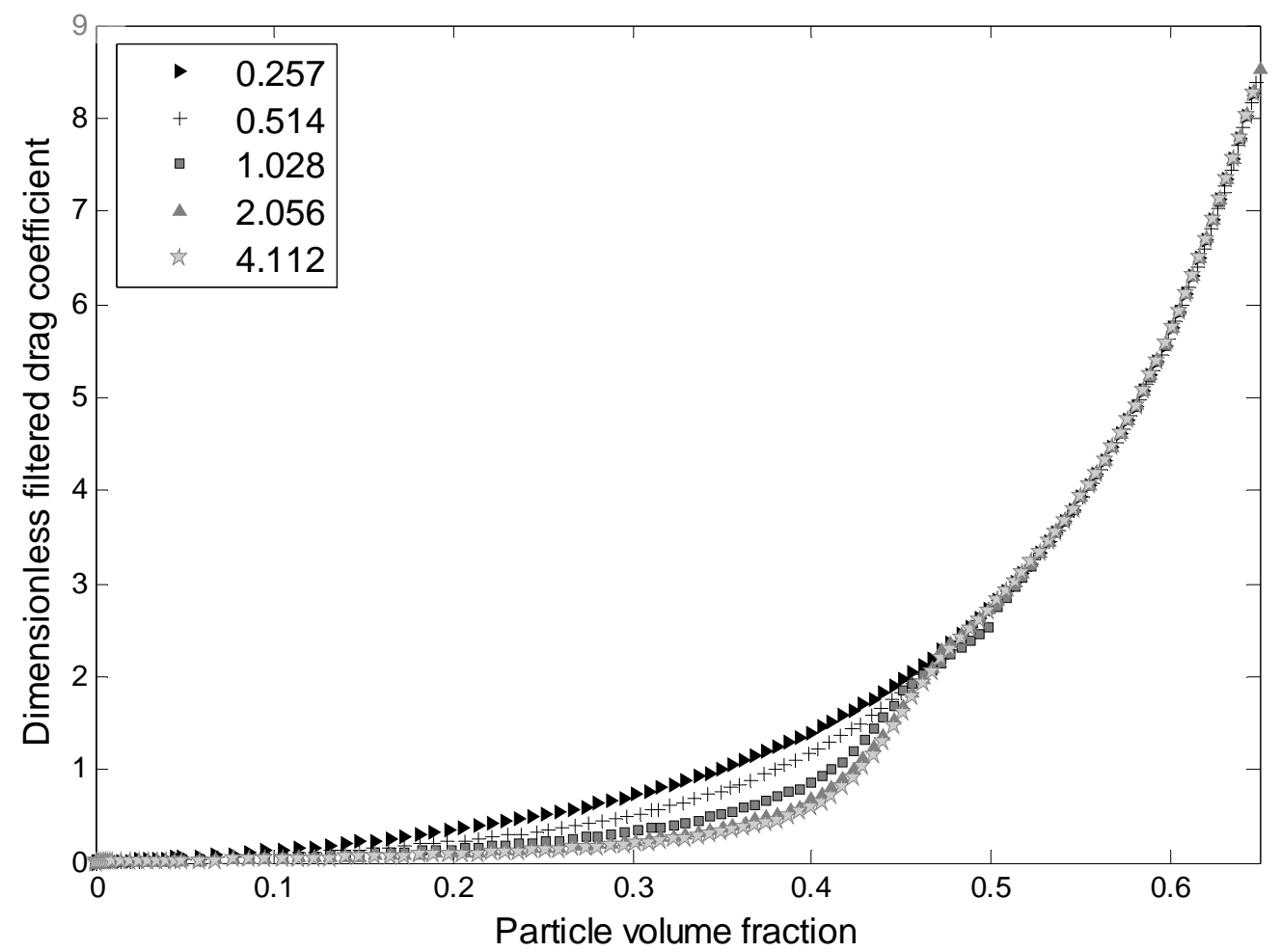

b)

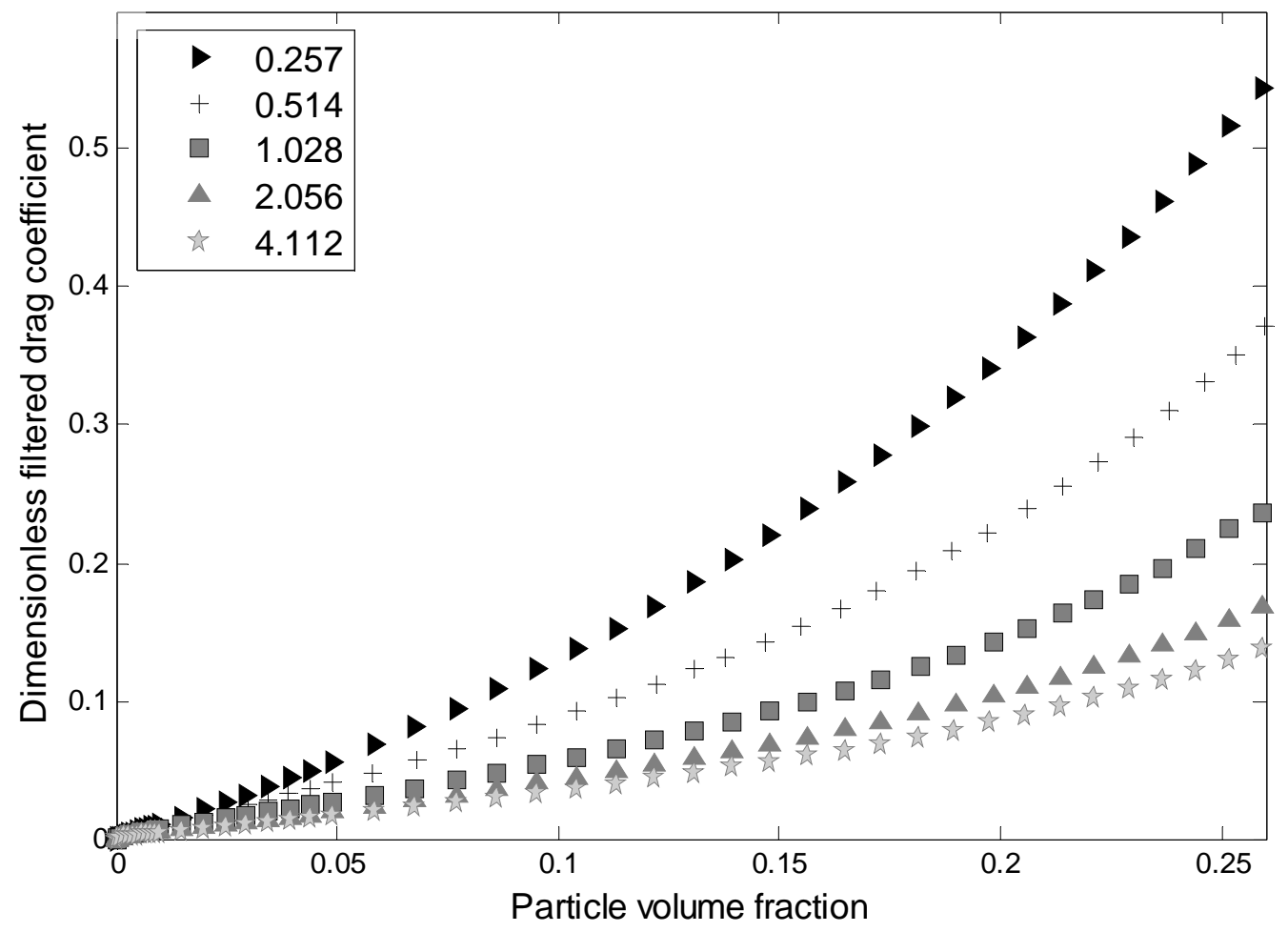


c)

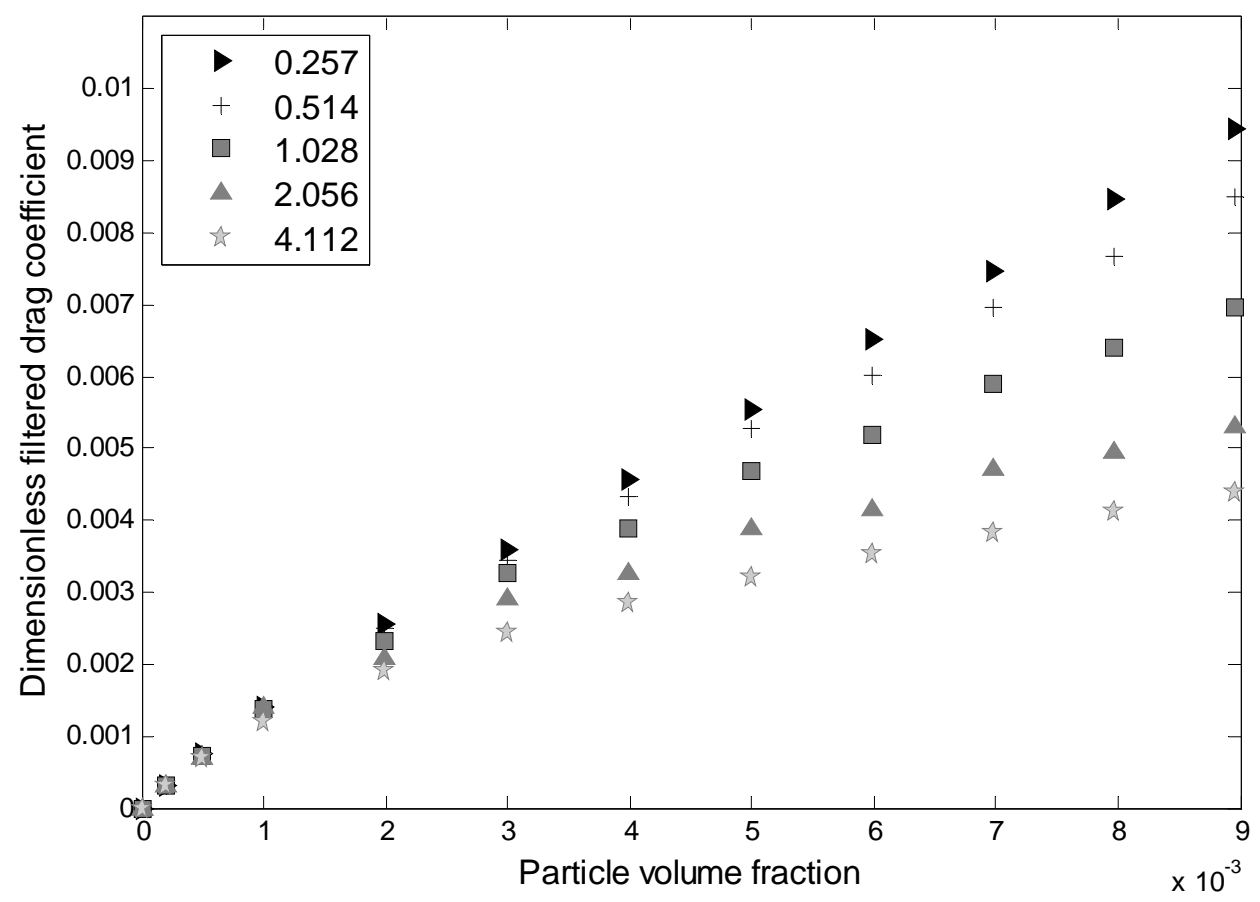

Figure 13: The variation of the dimensionless 3-D filtered drag coefficient with particle volume fraction for various filter lengths (listed in the legend in dimensionless units) is shown. Simulations were in a cubic domain of size 16.448 × 16.448 x 16.448 dimensionless units, using 64 x 64 x 64 grid points. The dimensionless filtered drag coefficient includes contributions from the drag force and the pressure fluctuation force. Data used for filtering were generated by running simulations for domain-average particle volume fractions of $0.01,0.02,0.05,0.10,0.15,0.20,0.25,30,0.35,0.40,0.45,0.50,0.55$ and 0.58. (a) The entire particle volume fraction range $\left(0 \leq \phi_{s}<0.65\right)$; (b) for $0 \leq \phi_{s}<0.25$ (to show the filtering effect more clearly in the regions of moderate particle concentration); (c) for the dilute volume fraction region $\left(0 \leq \phi_{s}<0.01\right)$. 
a)

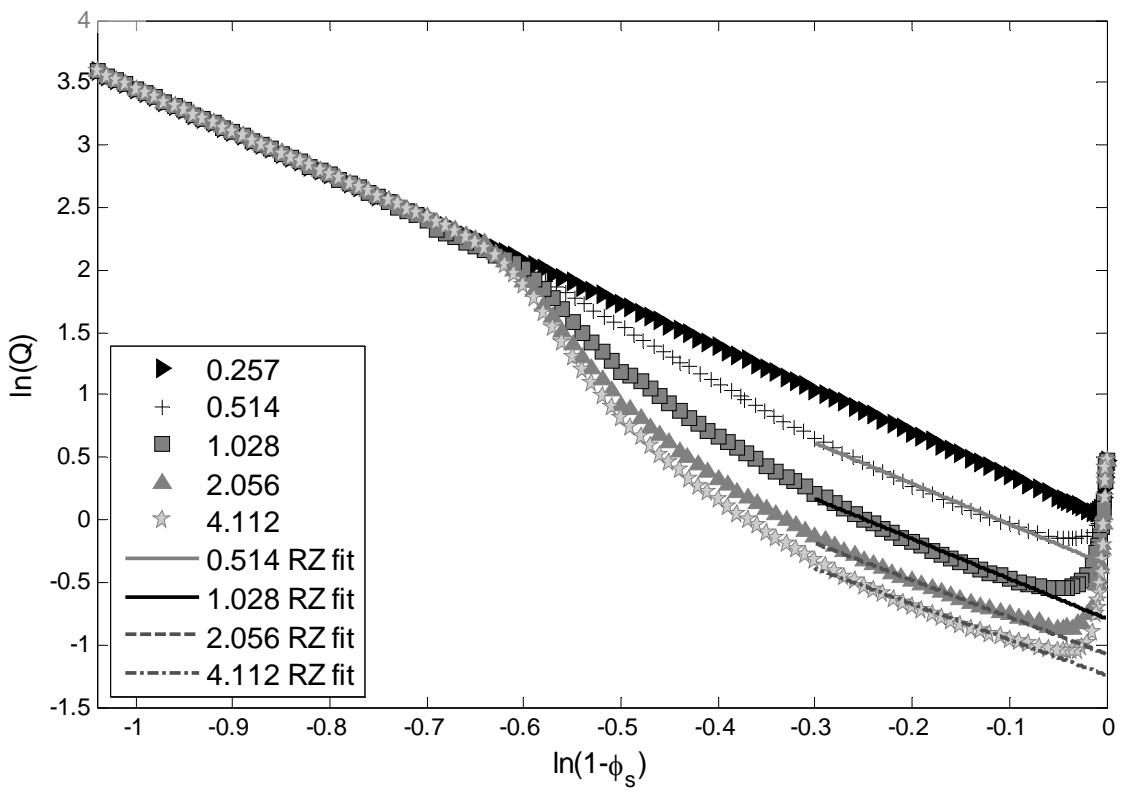

b)

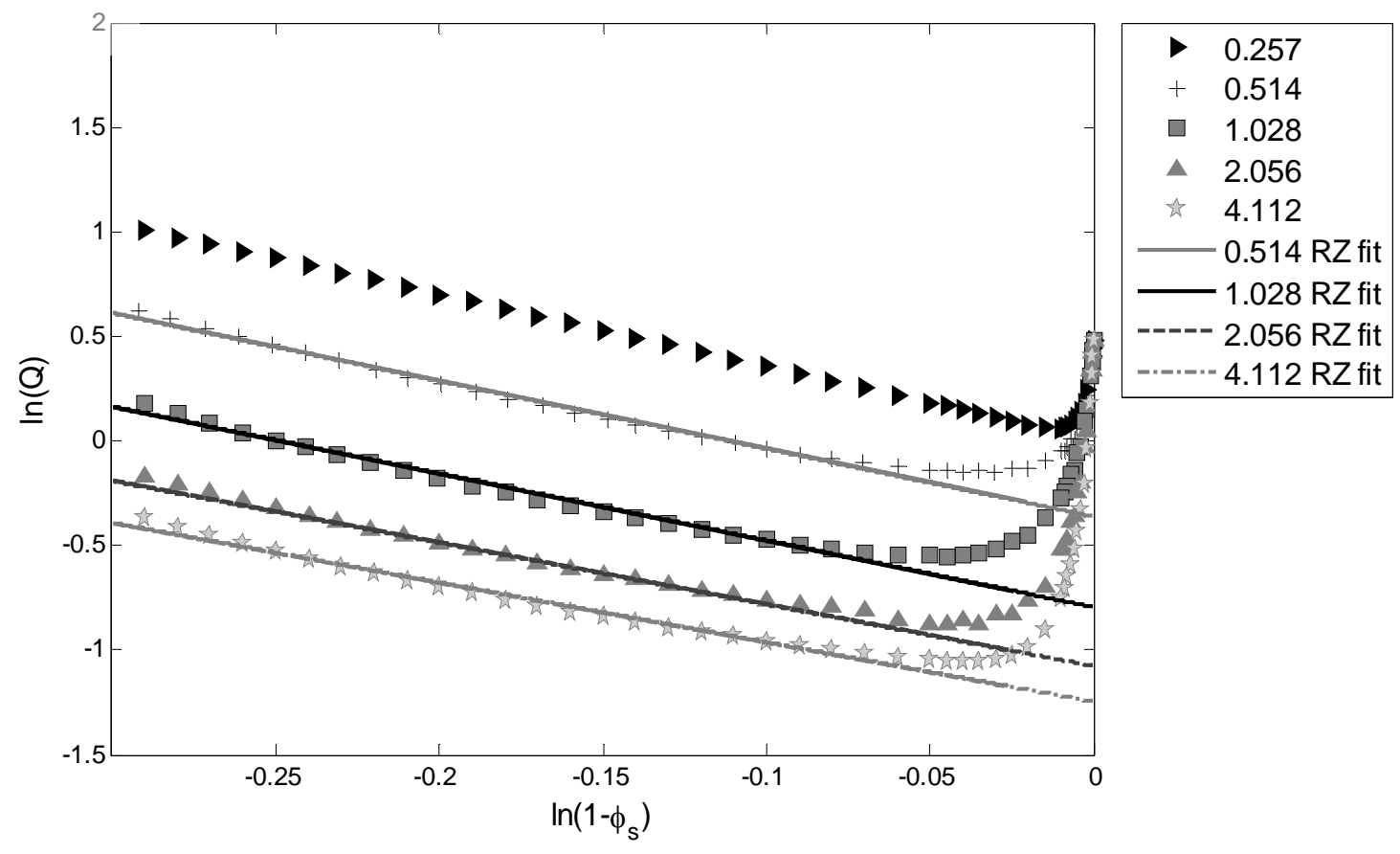


Figure 14: The results shown earlier in Figure 13(a-b) are plotted on a natural logarithmic scale. Here, $Q=\frac{\overline{\beta_{\text {filtered }, d}}}{\phi_{s}\left(1-\phi_{s}\right)}$, where $\overline{\beta_{\text {filtered }, d}}$ is the dimensionless filtered drag coefficient, $\phi_{s}$ is particle volume fraction. The dimensionless filter lengths are shown in the legend. (a) For the entire particle volume fraction range $\left(0 \leq \phi_{s}<0.65\right)$. (b) For $\phi_{s}<0.25$ (in order to show the filtering effect more clearly). A Richardson-Zaki (RZ) like behavior at moderate particle volume fraction is readily apparent, and these fits are also shown in the figure. However, the behavior in low and dense particle volume fraction regions is dramatically different and cannot be captured with this type of modeling. 


\section{a)}
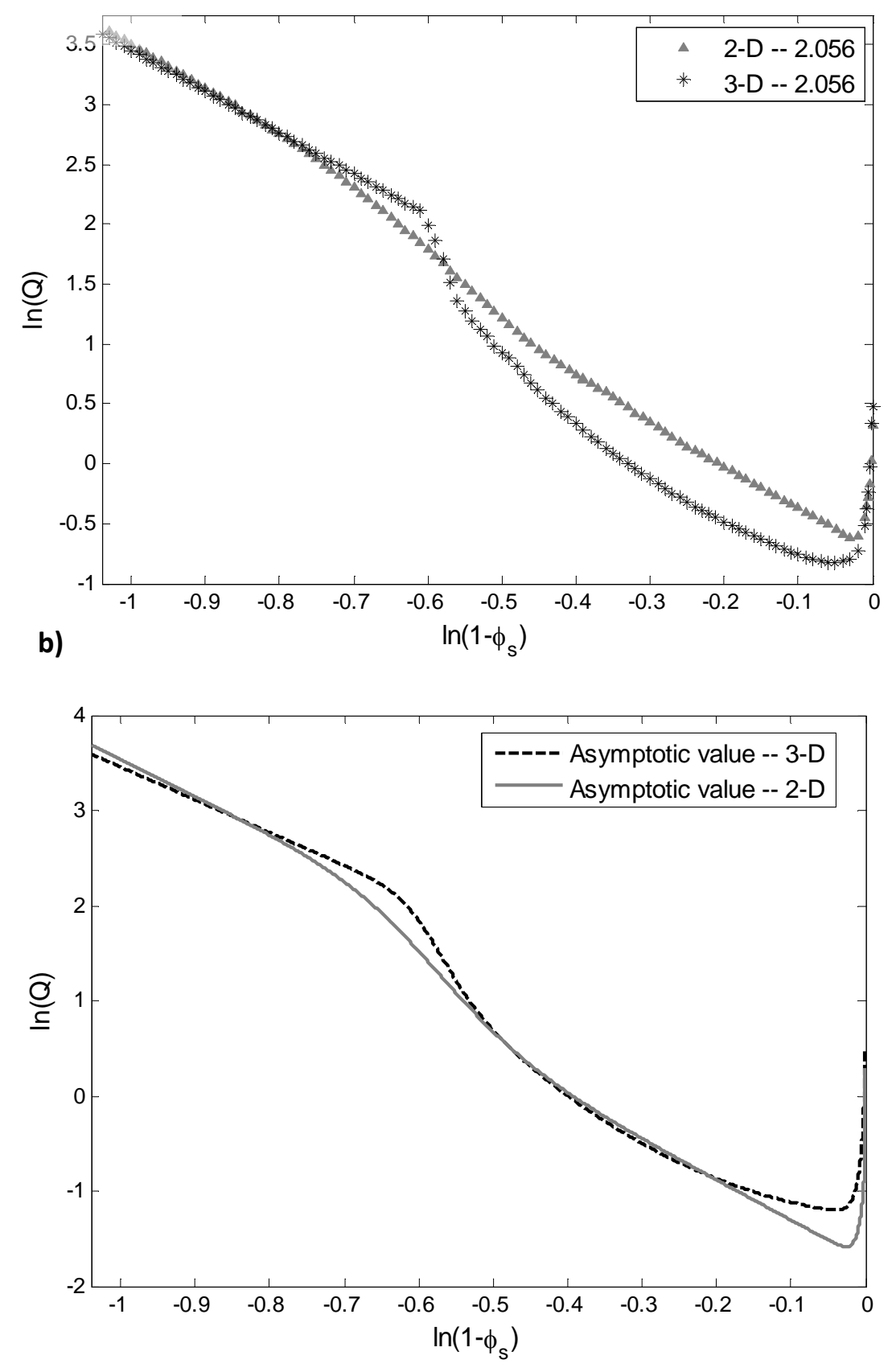

Figure 15: The comparison of $Q=\overline{\beta_{\text {filtered }, d}} / \phi_{s}\left(1-\phi_{s}\right)$ (on a natural logarithmic scale) extracted from 2$\mathrm{D}$ and 3-D simulations for (a) a filter length of 2.056 dimensionless units; (b) large filters (asymptotic value). 
a)

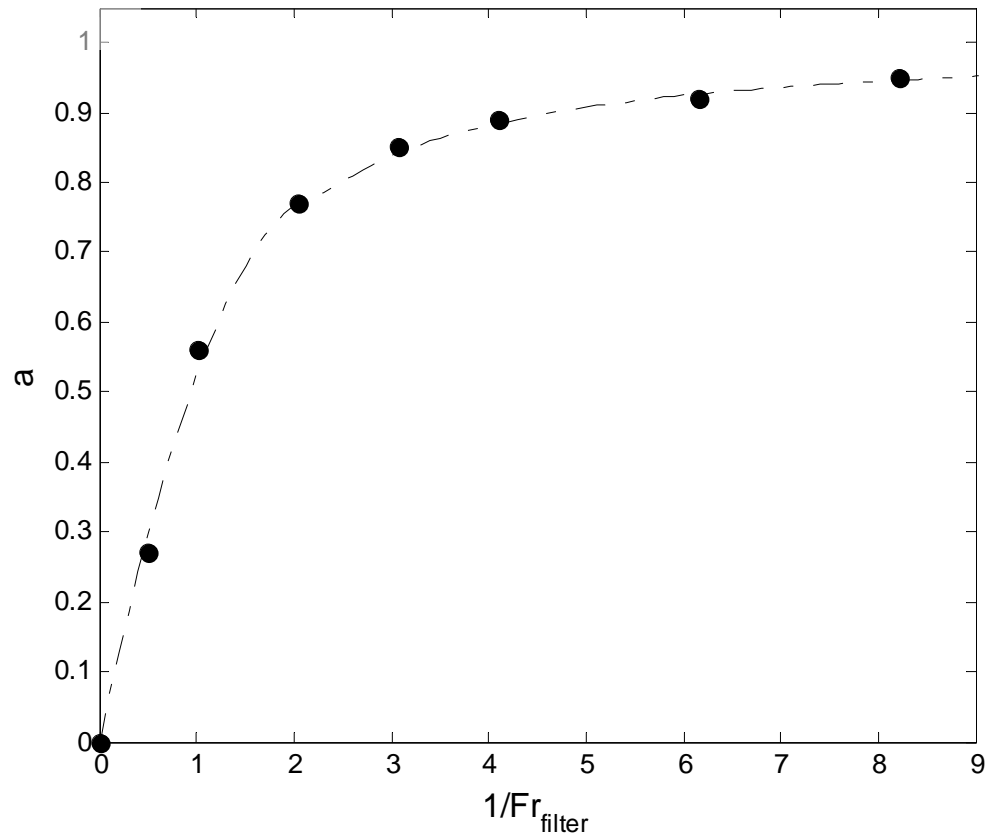

b)

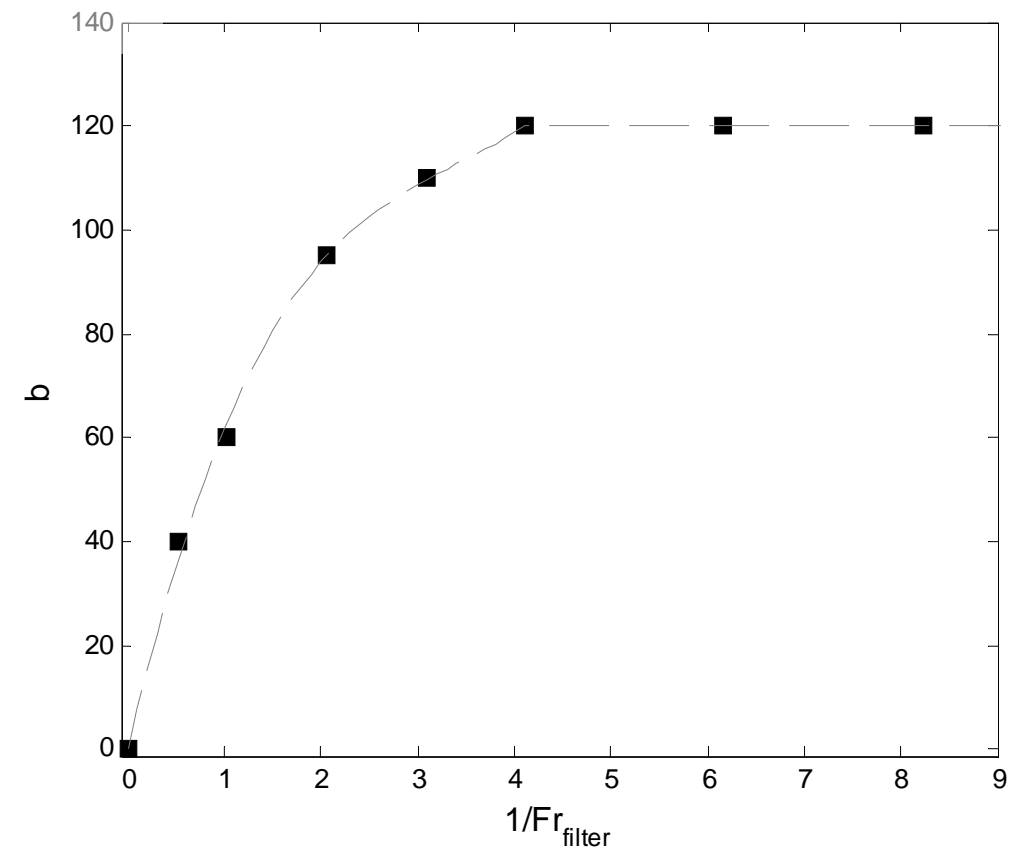

Figure 16: The variation of (a) the 3-D weighting function "a"; (b) the 3D weighting function exponent "b" with dimensionless filter length. "a" and "b" can be modeled as shown in Equation T3.6 (represented with the dash-dotted line) and Equation T3.9 (represented with the dashed line), respectively. See Table 3. 
a)

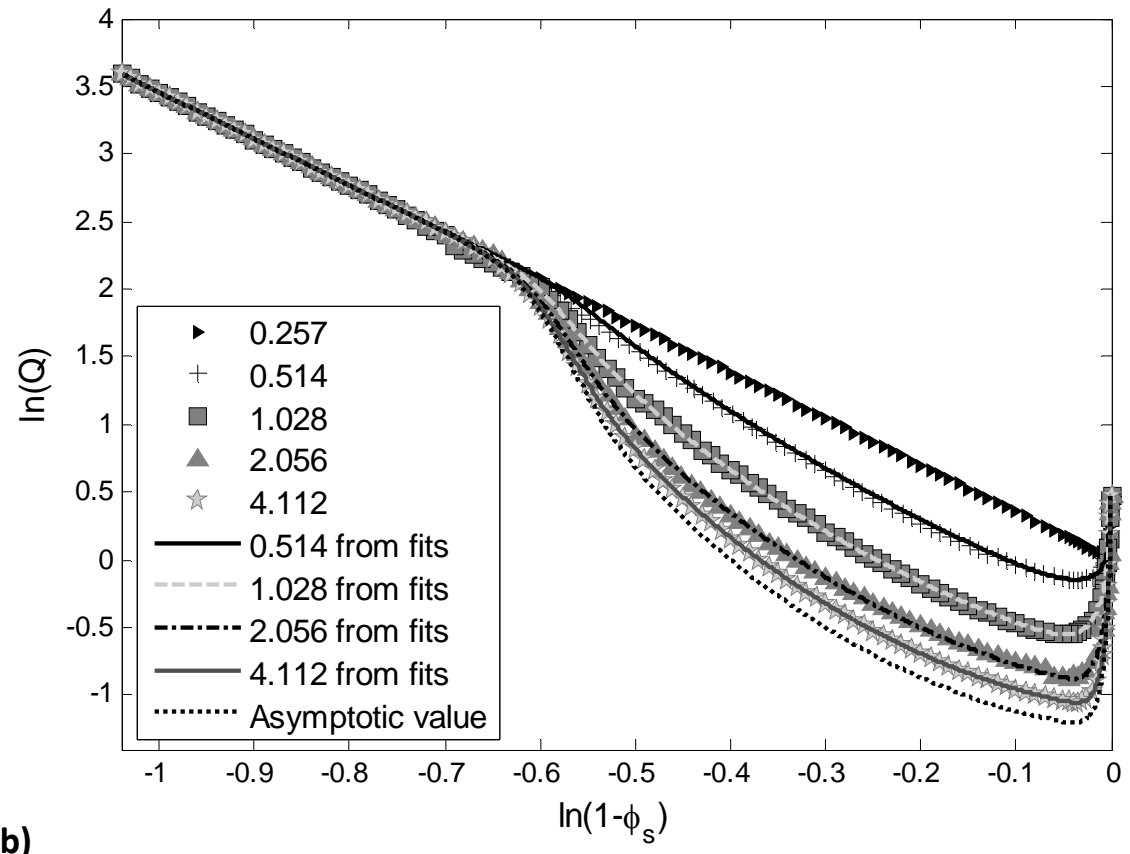

b)

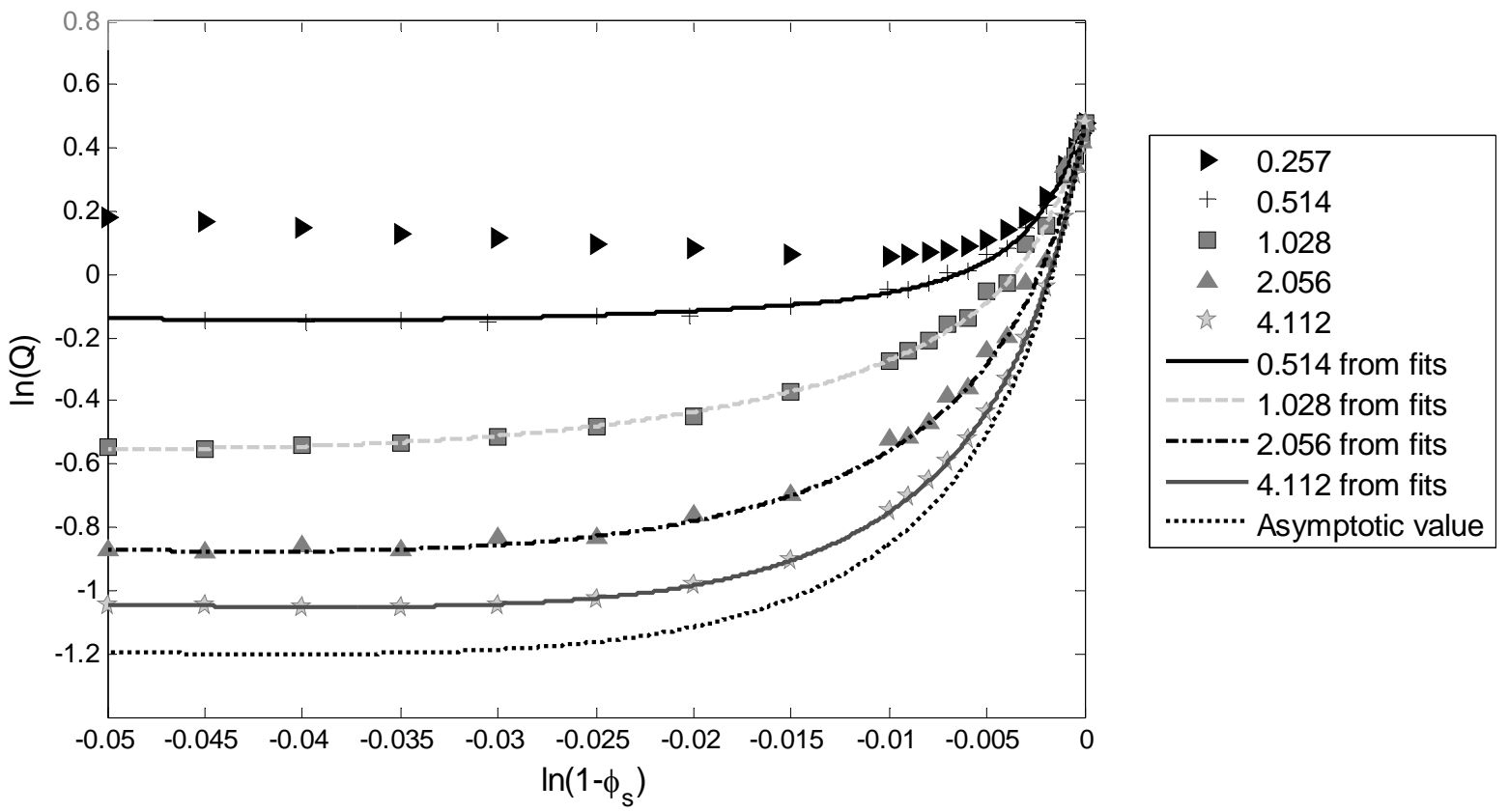




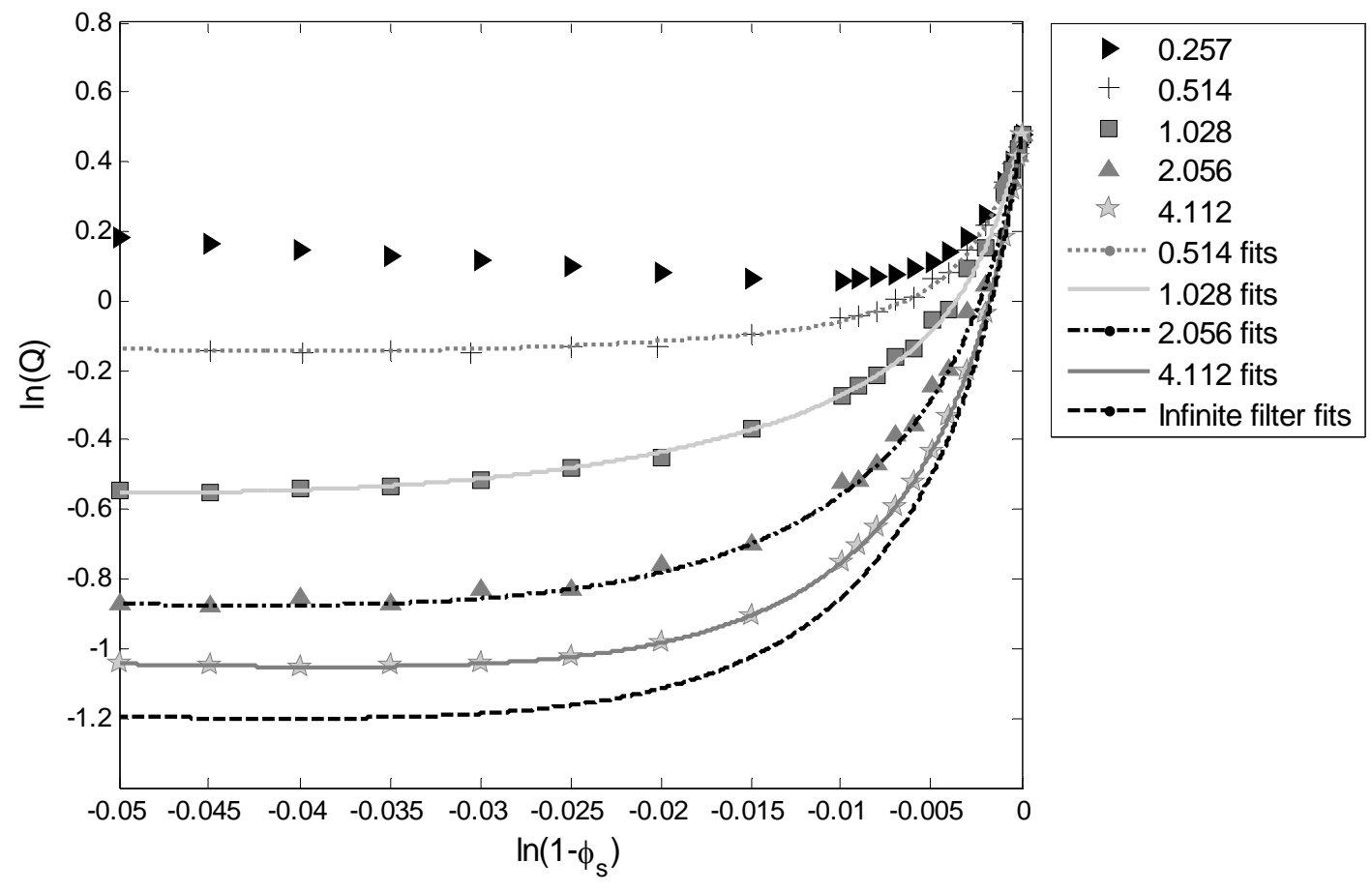

Figure 17: The results $(\ln (\mathrm{Q}))$ shown in Figure 13 are re-plotted to present a comparison with the constitutive model (as a function of particle volume fraction and filter length) shown in Equation T3.2 in Table 3. The bottom line indicates asymptotic value of $\ln (\mathrm{Q})$, which was obtained from scaling studies. (a) For the entire particle volume fraction range $\left(0 \leq \phi_{s}<0.65\right)$. (b) For $\phi_{s}<0.25$ (in order to show the filtering effect more clearly). 


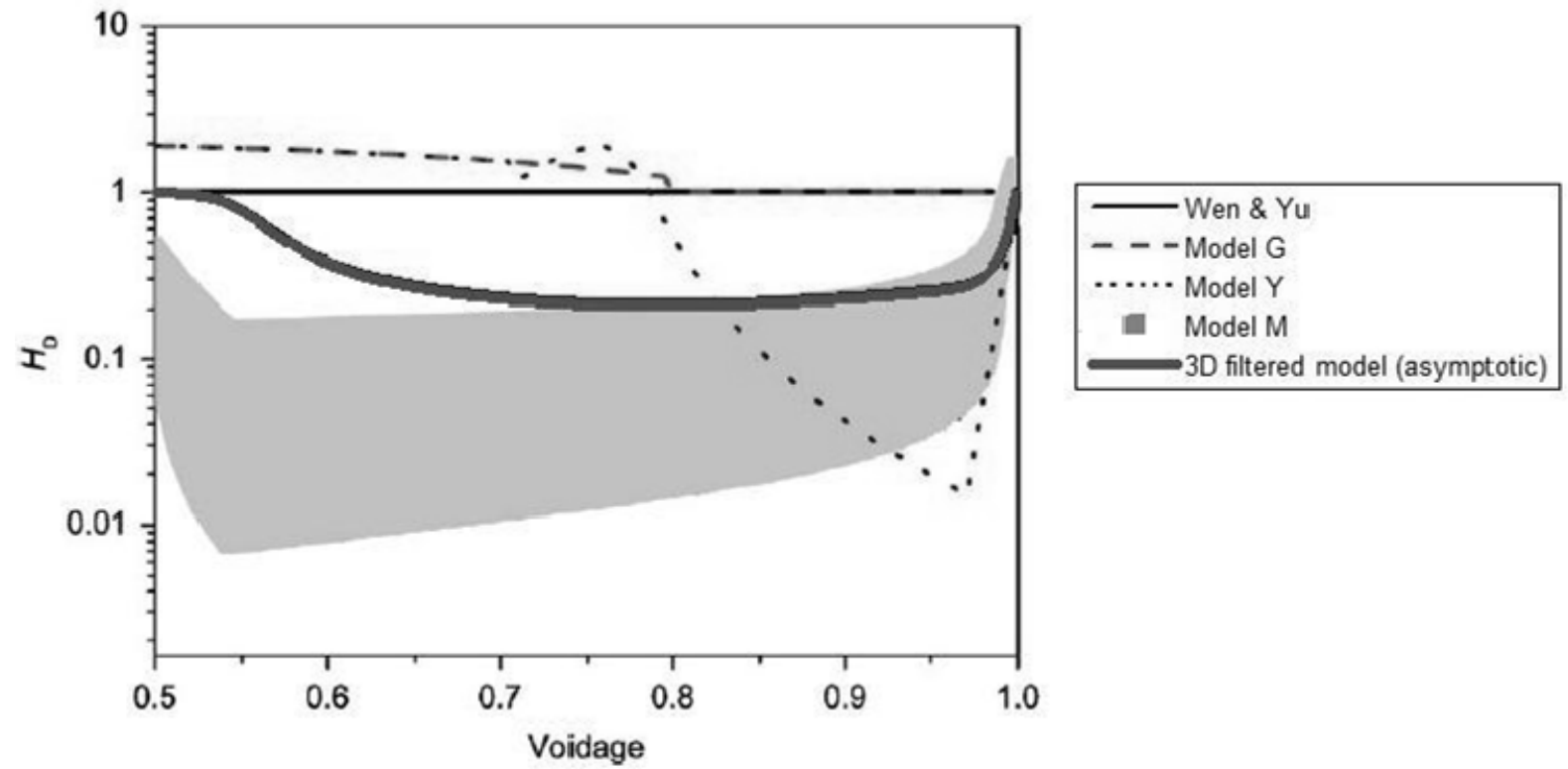

Figure 18: A comparison of the heterogeneity index, $H_{D}\left(=\beta_{\text {effective }} / \beta_{\text {Wen \& Yu }}\right)$, from the asymptotic value for 3-D filtered drag coefficient (for very large filters) with that from the EMMS/matrix model is presented. Here, we have used Figure 1 from Lu et al. ${ }^{15}$, which shows the projected area plot of $H_{D}$ (a function of $\phi_{s}$ and $V_{\text {slip }}$ ) from the EMMS/matrix model (Model M), $H_{D}$ from the previous version of EMMS model (Model Y), and that from the Ergun/Wen-Yu (Model G) and incorporated our filtered 3-D model in the figure for comparison. Wen and $\mathrm{Yu}$ drag coefficient was used as the standard drag coefficient, and the remaining models were scaled with it to get the corresponding $H_{D}$. The area plot shown in this figure covers a range of $0.001<V_{\text {slip }}<15.2 \mathrm{~m} / \mathrm{s}$. 
a)

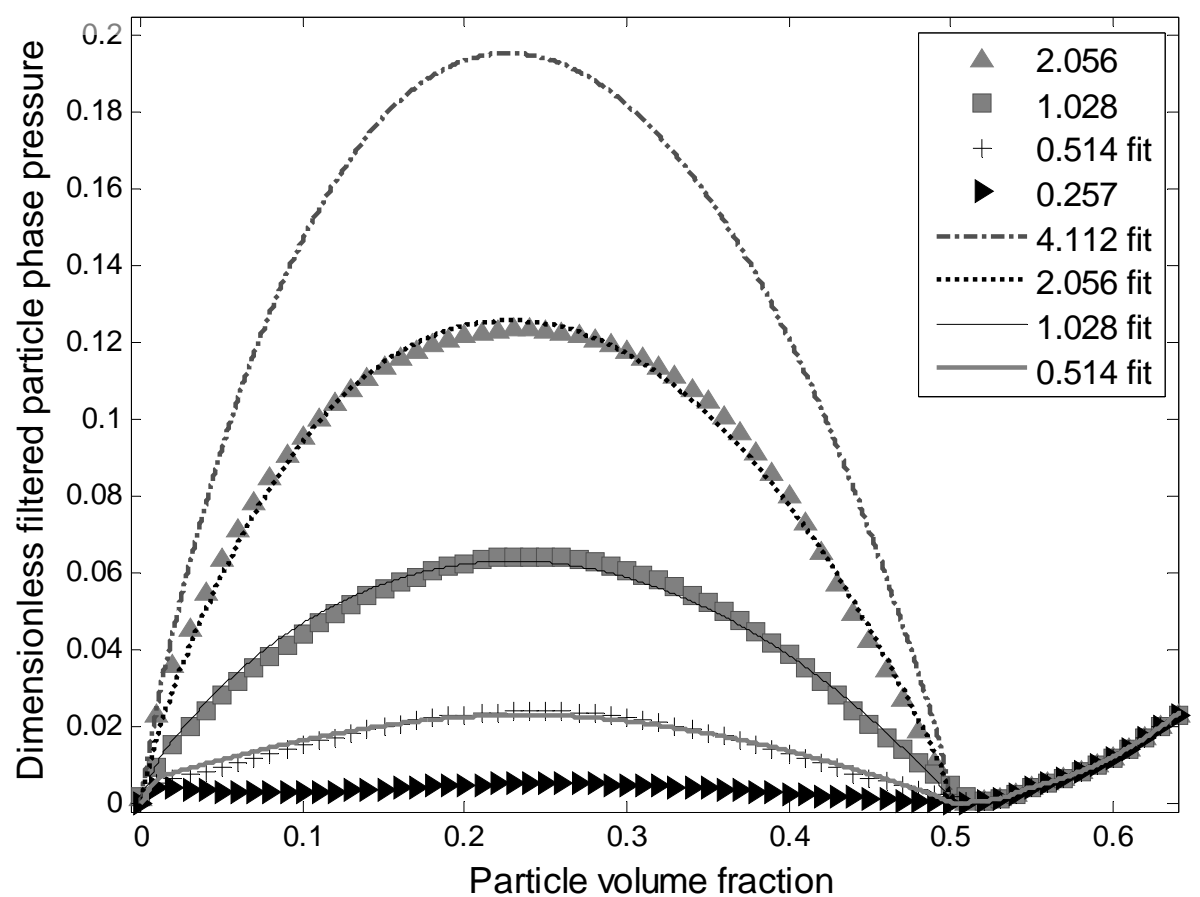

b)

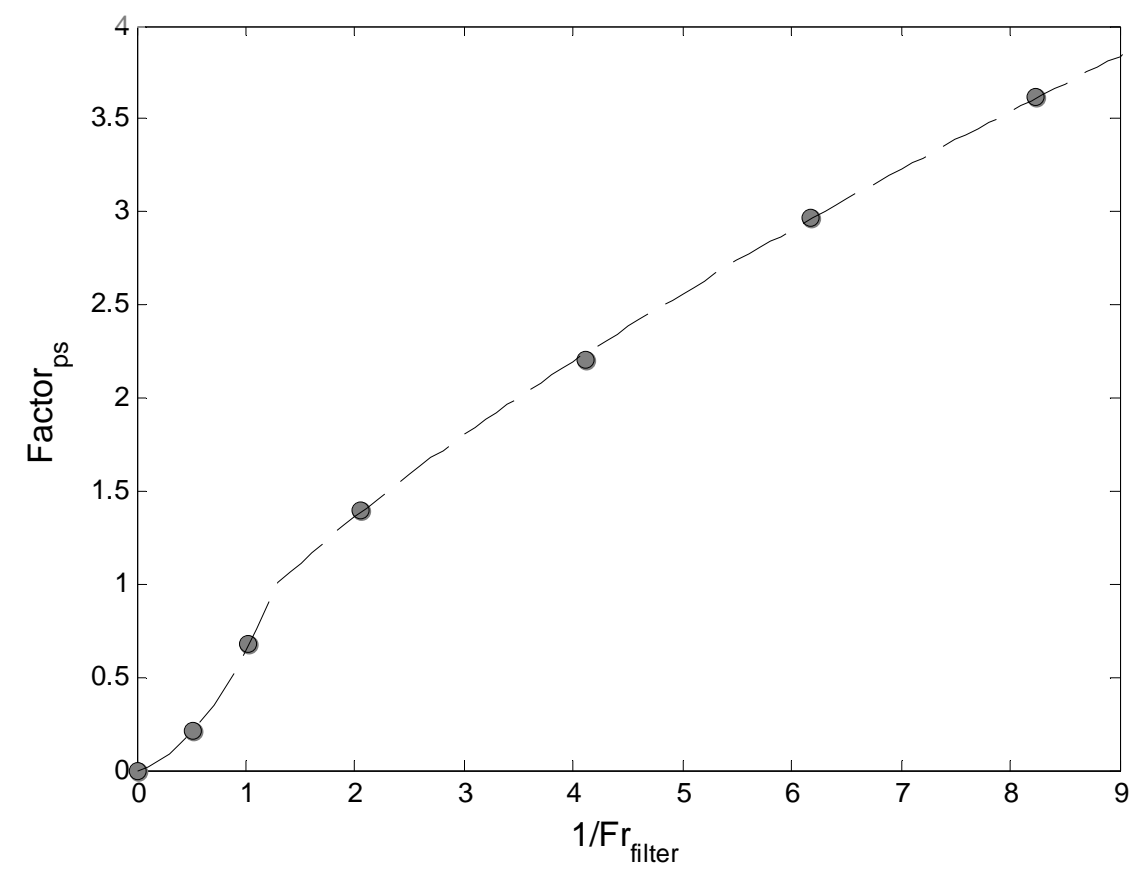

Figure 19: (a) The variation of the dimensionless 3-D filtered particle phase pressure with particle volume fraction is presented for various dimensionless filter lengths shown in the legend. The figure includes the constitutive relationships that capture the filtered particle phase pressure, see Equation T3.11. (b) The coefficient "Factor ps" in Equation T3.11 is plotted against the dimensionless filter length. "Factor ps" can be represented by Equation T3.13 in Table 3 (shown with the dashed line). 
a)

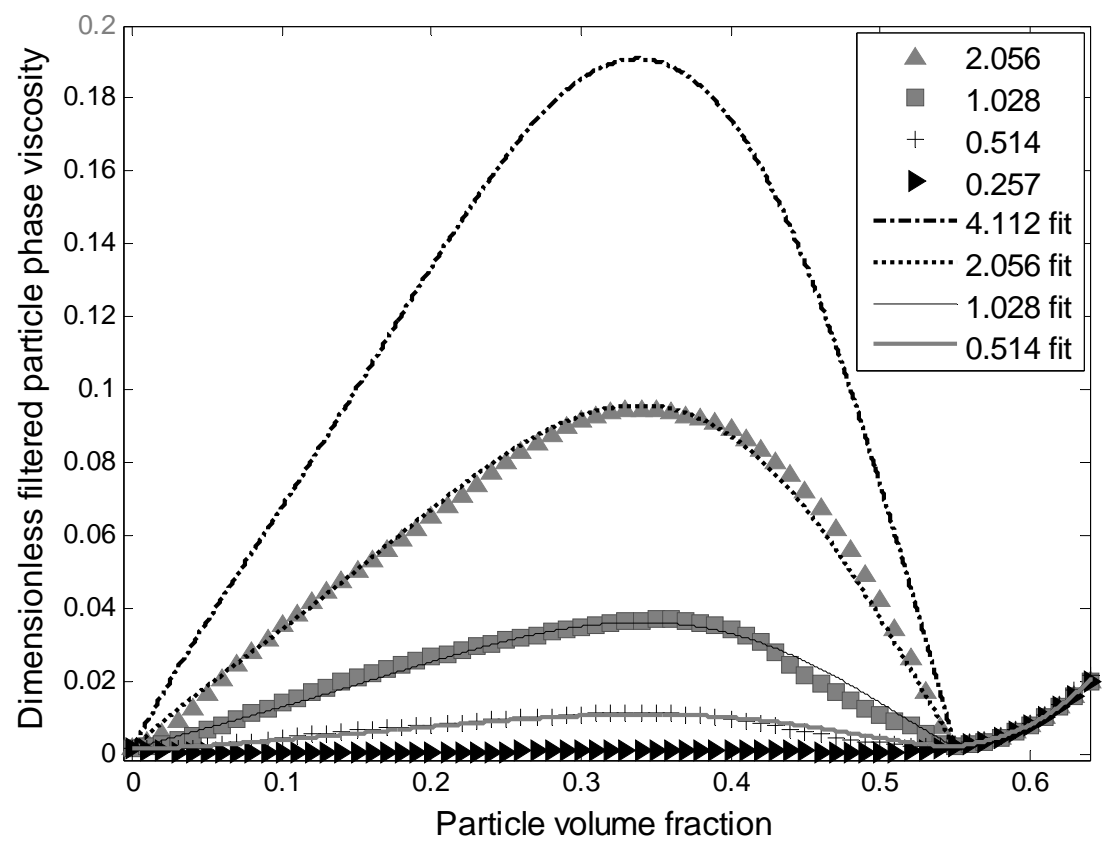

b)

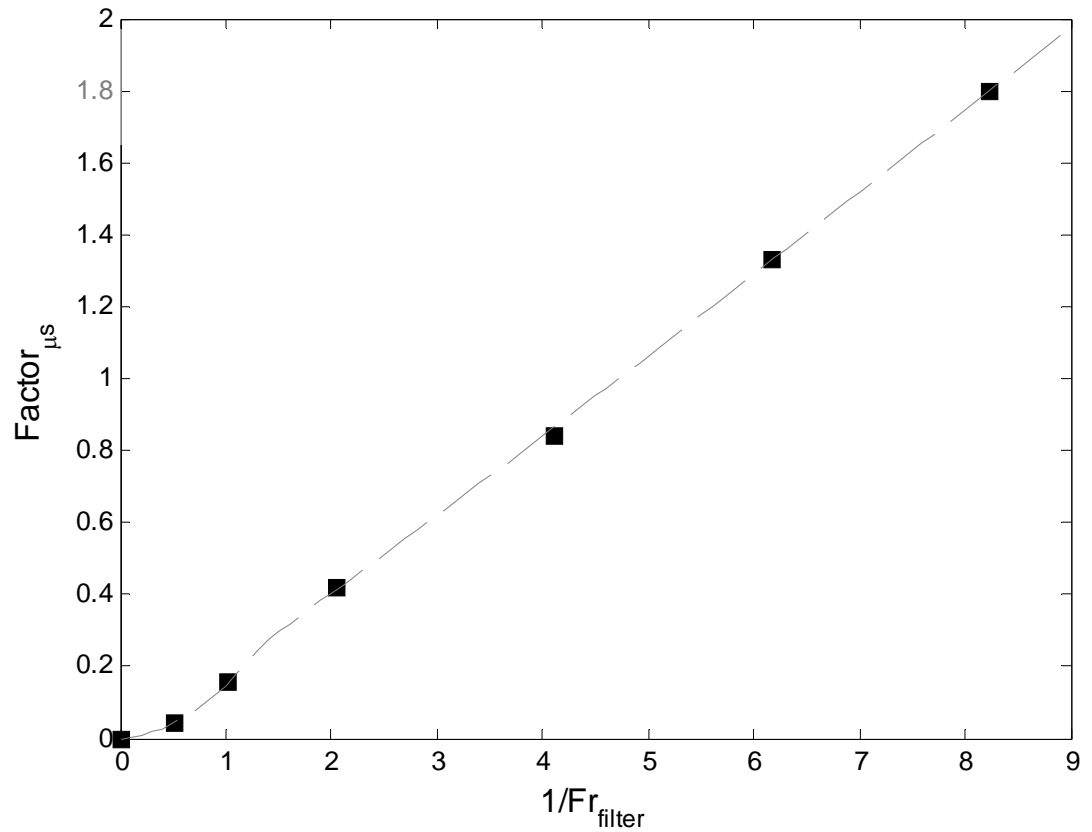

Figure 20: (a) The variation of the dimensionless 3-D filtered particle phase viscosity with particle volume fraction is presented for various dimensionless filter lengths. The results were extracted from simulations mentioned in the caption for Figure 13 (a-c). The dimensionless filter lengths are shown in the legend. This figure also includes the constitutive relationships that capture the filtered particle phase viscosity, see Equation T3.15. (b) The coefficient "Factor ${ }_{\mu \mathrm{s}}$ " in Equation T3.15 is plotted against the dimensionless filter length. "Factor ${ }_{\mu \mathrm{s}}$ " can be represented by Equation T3.17 in Table 3 (shown with the dashed line). 
a)

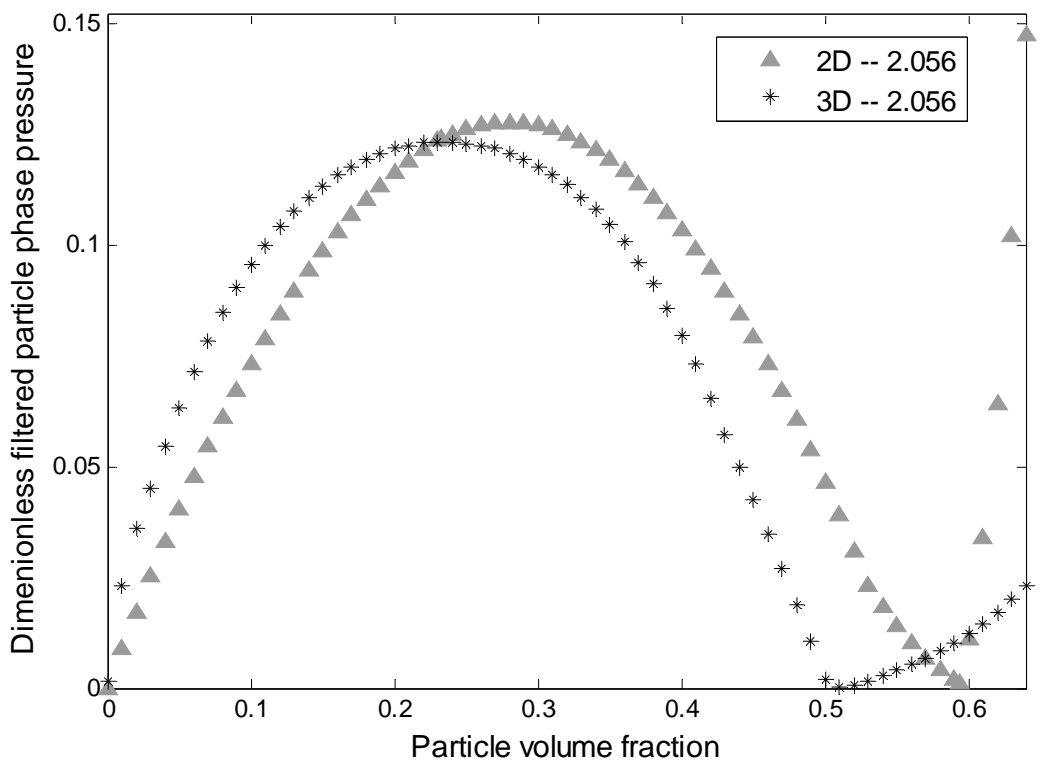

b)

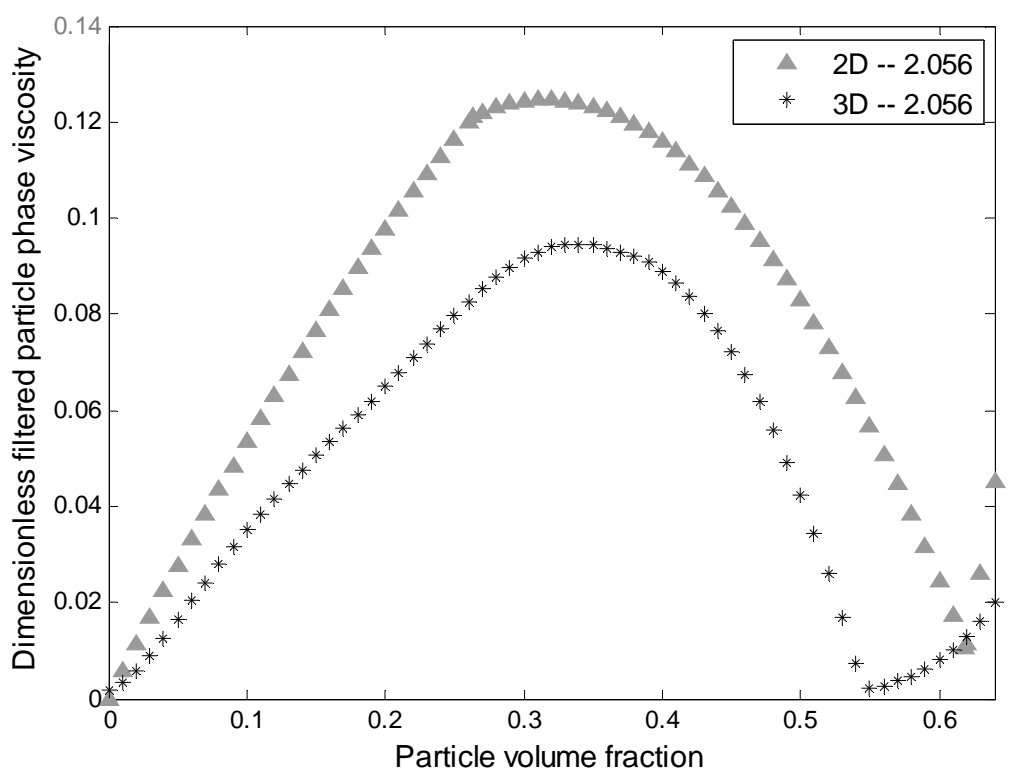

Figure 21: The comparison of (a) the filtered particle phase pressure (b) the filtered particle phase shear viscosity extracted from 2-D and 3-D simulations for a filter length of 2.056 dimensionless units. 